\title{
Integrated Estimation of Commercial Sector End-Use Load Shapes and Energy Use Intensities, Phase II
}

\author{
Final Report \\ Appendices \\ January 1991 \\ Prepared for \\ California Energy Commission \\ and \\ California Institute for Energy Efficiency \\ Prepared by \\ Hashem Akbari, Leo Rainer, and Joseph Eto \\ Energy Analysis Program \\ Applied Science Division \\ Lawrence Berkeley Laboratory \\ University of California \\ Berkeley, CA 94720
}

This report has been reproduced directly from the best available copy.

This research was sponsored by the California Energy Commission through the California Institute for Energy Efficiency (CEC Contract No. 300-88-004), and by the Assistant Secretary for Conservation and Renewable Energy, Office of Building and Community Systems of the U.S. Department of Energy under Contract No. DE-AC03-76SF00098. 
Table of Contents

Title

Description

Page

Abstract .................................................... i

Executive Summary ........................................ ii

List of Tables ................................................. viii

List of Figures ................................................ $\quad x$

Chapter 1

Introduction ................................................ 1

Chapter II

Methodology and Data.................................... 4

Chapter III Prototype Building Descriptions

and DOE-2 Building Simulations ........................ 14

Chapter IV

Whole-Building Load Shapes............................. 34

Chapter V

Reconciling End-Use EUls and Load Shapes ......... 54

Chapter VI

Developing EUls for the CEC Forecasting Model ...

97

Chapter VII

References

\section{Appendices (under separate cover)}

Appendix A

Appendix B

Appendix C

Appendix D

Appendix $E$
Memo on Re-examination of Phase I EUls for

Large Office, Large Retail, and Small Retail

and Replacement Tables for LBL-27512.

DOE-2 BDL Prototype Input Files

Summaries of LRD.

60

Results from Load-Temperature Regressions.

79

THI Matrices for Cooling

96 
Appendix A. Memo on Re-examination of Phase I EUls for Large Office, Large

Retail, and Small Retail and Replacement Tables for LBL-27512 


\section{CEC EOI/LS Phase II Project}

Revised End Use EUIS and Load Shapes for:

Large Office, Large Retail, and Small Retail

Discussion Notes Prepared for CEC Review

(May 8, 1990)

One of the outstanding issues from the Phase I project was the validity of the resultant end-use EUIs and load shapes for small office, large office, large retail, and small retail buildings. Phase I resultant EUIs for these building categories were considered at variance compared to other utility data. The EUIs of the Phase I project was obtained from utility bills of a sample of 375 buildings in Southern California. The average wholebuilding electric EUIs calculated from the sample utility bills were different for these building types. A reanalysis of the end-use EUIS and LSS for these three buildings was postponed to Phase II (the current Phase) of the project.

As we have discussed in the Phase I Einal Report, whole-building EUIs are "control" figures for the reconciliation process; they are used to estimate end-use EUIs and ISs subject to the condition that the total of all end-use EUIs for a building type would be the same as the controlling whole-building EUIs. The whole-building EUIs were calculated by weighting and averaging the electric bills for the on-site survey premises. Small sample sizes and uncertain weights may have led to whole-building EUIs for these building types that were different from other utility data sources.

In collaboration with CEC project staff, we have reviewed the available whole-buildings EUIs for these building categories in order to arrive at a set of acceptable whole-building EUIs for 
the reconciliation process. These notes summarize our conclusions. We have also carried out the process of reconciliation for these buildings and obtained a new set of end-use EUIs and LSS .

\section{Whole-Building EUIs}

As we have stated in our Eebruary 23, 1990 memo to CEC, several sources for whole-building EUIs were considered including: LBL analysis of the SCE 1985 mail survey, CEC analysis of the SCE 1988 mail survey, CEC analysis of SCE 1987 and 1988 QEER data, and SCE analysis of its 1988 accounts by SIC code (provided by Tim Tutt on February 22, 1990). The following whole-building EUIs were adopted:

\section{Small Retail $12.2 \mathrm{kWh} / \mathrm{sf}$ \\ Large Retail $18.2 \mathrm{kWh} / \mathrm{sf}$}

Although, initially, it was planned to reanalyze the small office buildings, at the February 23 meeting, it was decided not to proceed with the reanalysis. This decision was based on additional data supporting the Phase I whole-building EUI for small offices.

Eor large offices, LBI was charged to review the available data and prepare a memo proposing whole-building EUIs. This note is intended to replace that memo.

Available Data. SCE analysis of 1988 accounts (SI) estimates a whole-building EUI for large offices of $19.8 \mathrm{kWh} / \mathrm{sf}$. (SI also estimates a whole-building EUI for small offices of $12.6 \mathrm{kWh} / \mathrm{sf}$; we will use this data later on to justify our conclusions.) CEC estimates whole-building EUIs for all offices from 1986 (S2) and 1987 (S3) data of 16.2 and $16.5 \mathrm{kWh} / \mathrm{sf}$, respectively. CEC 
preliminary analysis of 1988 SCE mail data (S4) has resulted in a whole-building EUI for large offices of $11.0 \mathrm{kWh} / \mathrm{sf}$. (S4 also estimates a whole-building EUI for small offices of $15.9 \mathrm{kWh} / \mathrm{sf}$; we will use this data later on to justify our conclusions.) The following table summarizes these data sources

Code Description

S1 SCE 1988 accounts

S2 CEC 1986 analysis

S3 CEC 1987 analysis

S4 CEC 1988 analysis
Large Offices Small offices

All offices
12.6

16.2

16.5

11.0

15.9

Review and Recommendation. If we calculate the whole-building EUIS for all office buildings from $S 1$ and $S 4$, using the estimates of floor areas for large offices (241 Msf) and small offices ( $125 \mathrm{MSf}$ ) in the SCE service area, the resulting EUIs will be 17.4 and $12.7 \mathrm{kWh} / \mathrm{sf}$. A quick comparison of all-office EUIS from these sources indicate that difference between S1, S2, and $S 3$ are less than 7\%. However, the difference between $S 4$ and all other sources is $20 \%-27 \%$. (S1 is used as the basis for estimating percent differences.)

To understand these differences better, we reviewed the 54 data in further details. Cumulative frequency plot of the floor areas for large offices in this data set indicated that close to $35 \%$ of records are buildings with less than 10,000 sf; $25 \%$ are less than 6,500 sf; and $50 \%$ less than 23,000 sf (See Figure 1). There appears to be some errors in the data base which need t. be filtered. Cumulative frequency plots for the EUIs also indicated that about $25 \%$ of large offices have EUIs less than 6 $\mathrm{kWh} / \mathrm{sf}$ and about $10 \%$ have unusually large EUIs (> $50 \mathrm{kWh} / \mathrm{sf}$ ). 
To preliminary check the impact of these possible data errors on EUIs, we arbitrarily dropped the lower and upper $25 \%$ of the data points and noticed that the average EUI increased to 14.6 $\mathrm{kWh} / \mathrm{sf}$. A step in the right direction!

In summary, it is obvious that data source 54 needs to be cleaned up and cannot be considered as reliable. Also, the differences between S1, S2, and $S 3$ are fairly minimal. Therefore, to be consistent with sources for small and large retail whole-building EUIs, we propose using s1, EUI = $19.8 \mathrm{kWh} / \mathrm{sf}$, as the controlling whole-building eOI for reconciliation process.

\section{Results}

We applied the EDA, discussed in detail in Phase I final report, to obtain individual end use EUIs and LSs. Since, the whole building EUIs reflect the energy intensity of the entire SCE service area, we needed to adjust these EUIs to estimate EUIs for each climate zones. We used adjustment factors derived from DOE-2 simulations as discussed in phase $I$ final report. The adjustment factors by climate zones are:

$\begin{array}{lccc}\text { Building Type } & \text { LAX } & \text { BUR } & \text { NOR } \\ \text { Large Office } & 0.95 & 1.00 & 1.06 \\ \text { Large Retail } & 0.97 & 0.99 & 1.06 \\ \text { Small Retail } & 0.96 & 0.99 & 1.06\end{array}$

The adjusted whole-building EUIs are then used to estimate enduse EUIs and load shapes. Table 1 summarizes the 'new' end-use EUIs and compares them with those report in phase I report ('old'). The annual average daily load shapes for standard and non-standard days for these building types are estimated and 
plotted on Figures 2 (a\&b) for large offices, Figures 3 (a\&b) for large retails, and Figures 4 (asb) for small retails.

Upon CEC's review and approval of the 'new' EUIs and load shapes, we will calculate THI matrixes for these building types and transfer the data electronically to CEC. 


\begin{tabular}{|c|c|c|c|c|c|}
\hline \multicolumn{6}{|c|}{ 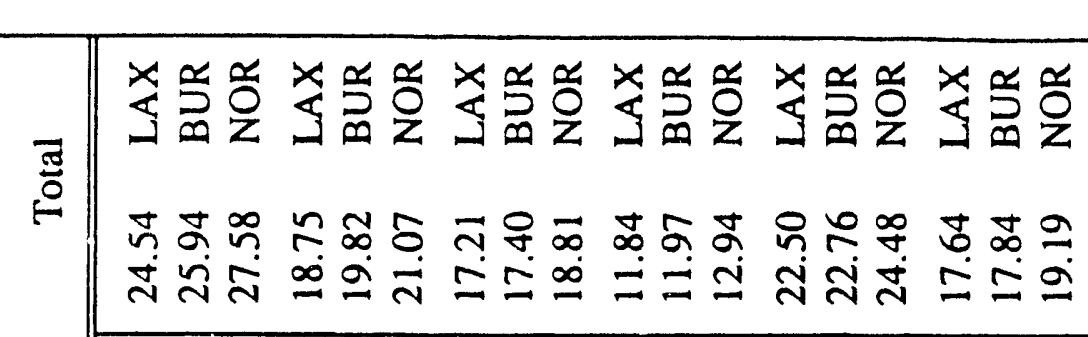 } \\
\hline \multicolumn{6}{|c|}{ 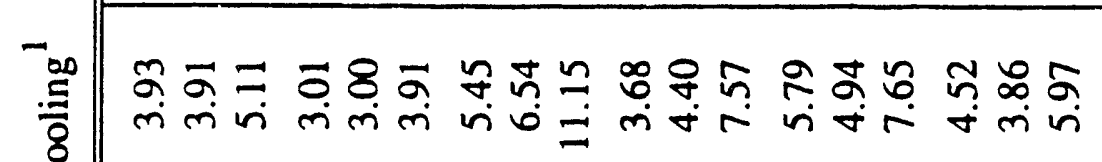 } \\
\hline \multicolumn{6}{|c|}{ 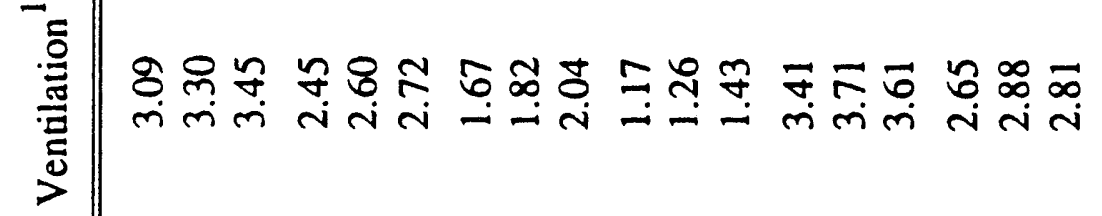 } \\
\hline 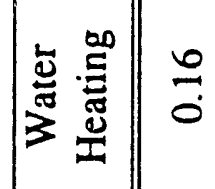 & $\frac{2}{\partial}$ & $\stackrel{\partial}{\partial}$ & : & छ & ร \\
\hline$\left.\frac{0}{3}\right)$ & $\frac{8}{8}$ & $\bar{\partial}$ & : & $\bar{\partial}$ & 亏े \\
\hline 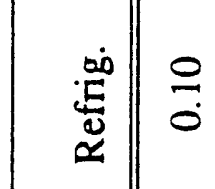 & $\%$ & 总 & 萼 & ¿ & g \\
\hline$\frac{4}{2}$ & $\bar{g}$ & $\stackrel{Ð}{ \pm}$ & $\bar{\sigma}$ & $\cong$ & 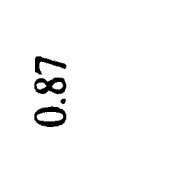 \\
\hline 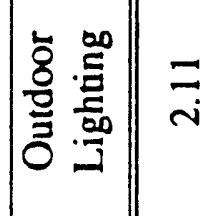 & $\underline{\Xi}$ & $\stackrel{8}{2}$ & $\stackrel{\dddot{g}}{\dddot{2}}$ & 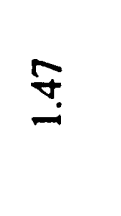 & $\Xi$ \\
\hline 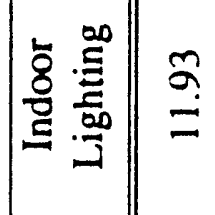 & $\stackrel{g}{\circ}$ & 2 & $\bar{\Sigma}$ & $\cong$ & \\
\hline & & & & & \\
\hline
\end{tabular}




\begin{tabular}{|c|c|c|c|c|c|c|}
\hline 䙲 & 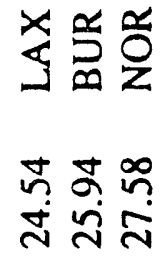 & 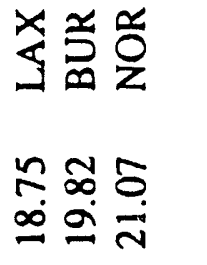 & 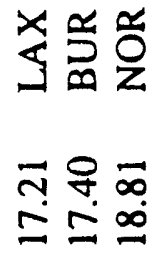 & 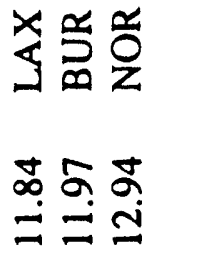 & 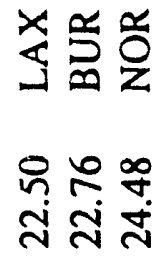 & 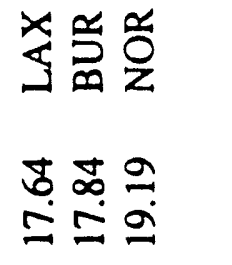 \\
\hline 告 & $\hat{\sigma} \bar{\sigma}=$ & जि & 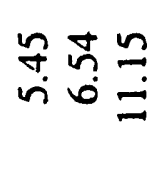 & 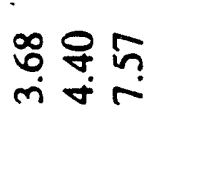 & 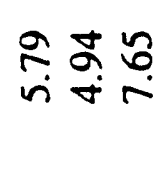 & ஸैळ \\
\hline & 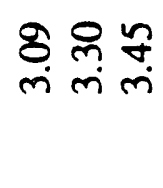 & 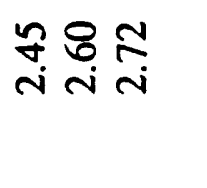 & نَّ & 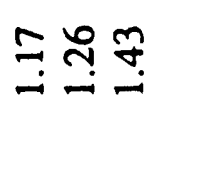 & $\vec{\nabla} \bar{\nabla} \bar{b}$ & 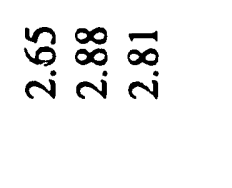 \\
\hline 总 & $\frac{0}{0}$ & $\frac{y}{0} \frac{m}{0} \frac{N}{0}$ & రั & ڤ̊ㅜㅇ & $\stackrel{\Im}{0}$ & đ̃ \\
\hline 告 & $\stackrel{8}{8}$ & $\begin{array}{l}8 \\
0 \\
0\end{array}$ & $\ddot{0}$ & 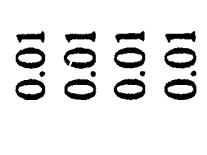 & $\frac{a}{0}$ & $\frac{5}{0} \frac{\infty}{0} \frac{1}{0}$ \\
\hline 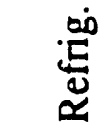 & $\stackrel{\circ}{\circ}$ & $\frac{0}{0} 8 \frac{0}{0} \frac{0}{0}$ & $\tilde{a}$ & ஊ̊̆ & $\overrightarrow{0}$ & 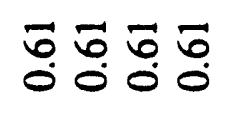 \\
\hline$\dot{\infty}$ & $\stackrel{\infty}{\dddot{\sim}}$ & 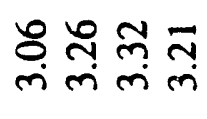 & $\stackrel{\infty}{-}$ & 뭉ㅎㅎ & $\cong$ & 成 \\
\hline مَ & $\bar{i}$ & 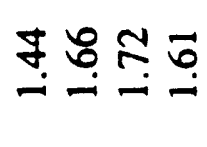 & $\stackrel{n}{n}$ & กี & f. & 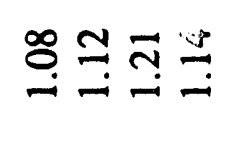 \\
\hline مَ & $\stackrel{\sigma}{a}$ & $\begin{array}{l}0 \\
\infty \\
\infty\end{array}$ & $\stackrel{g}{r}$ & 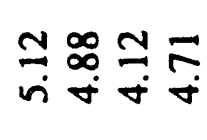 & 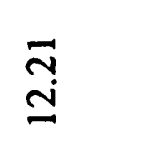 & $\begin{array}{l}\text { å윰 } \\
\text { a }\end{array}$ \\
\hline & 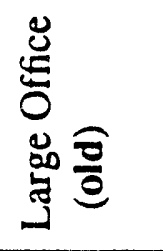 & 氖 & 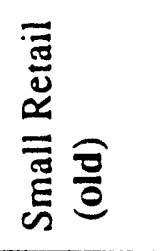 & $\widehat{E}$ & 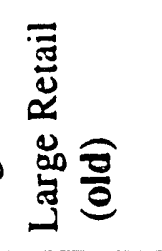 & 氖 \\
\hline
\end{tabular}

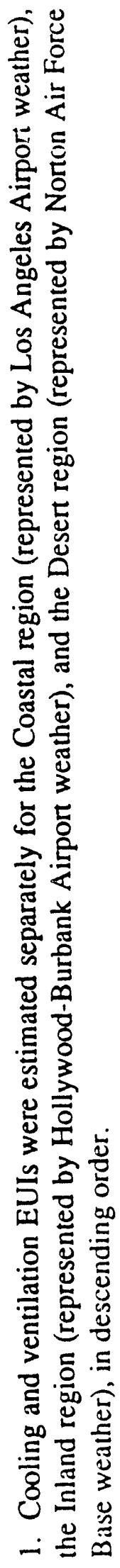




$$
\text { Fiy } 2 a
$$

\section{'Igoff'}

\section{'StandardDayLoadShapes'}

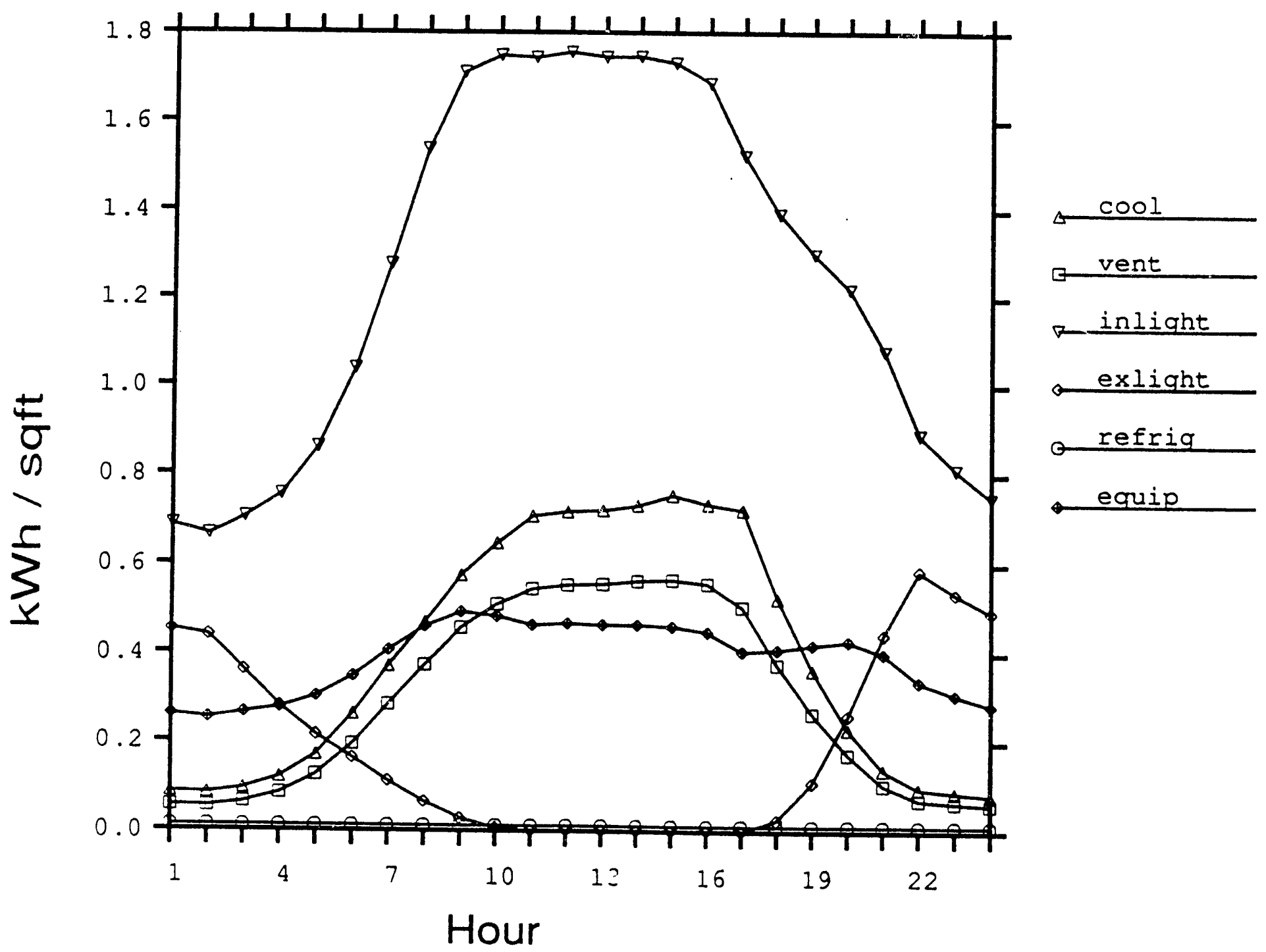




\section{'Igoff'}

'NonstandardDayLoadShapes'

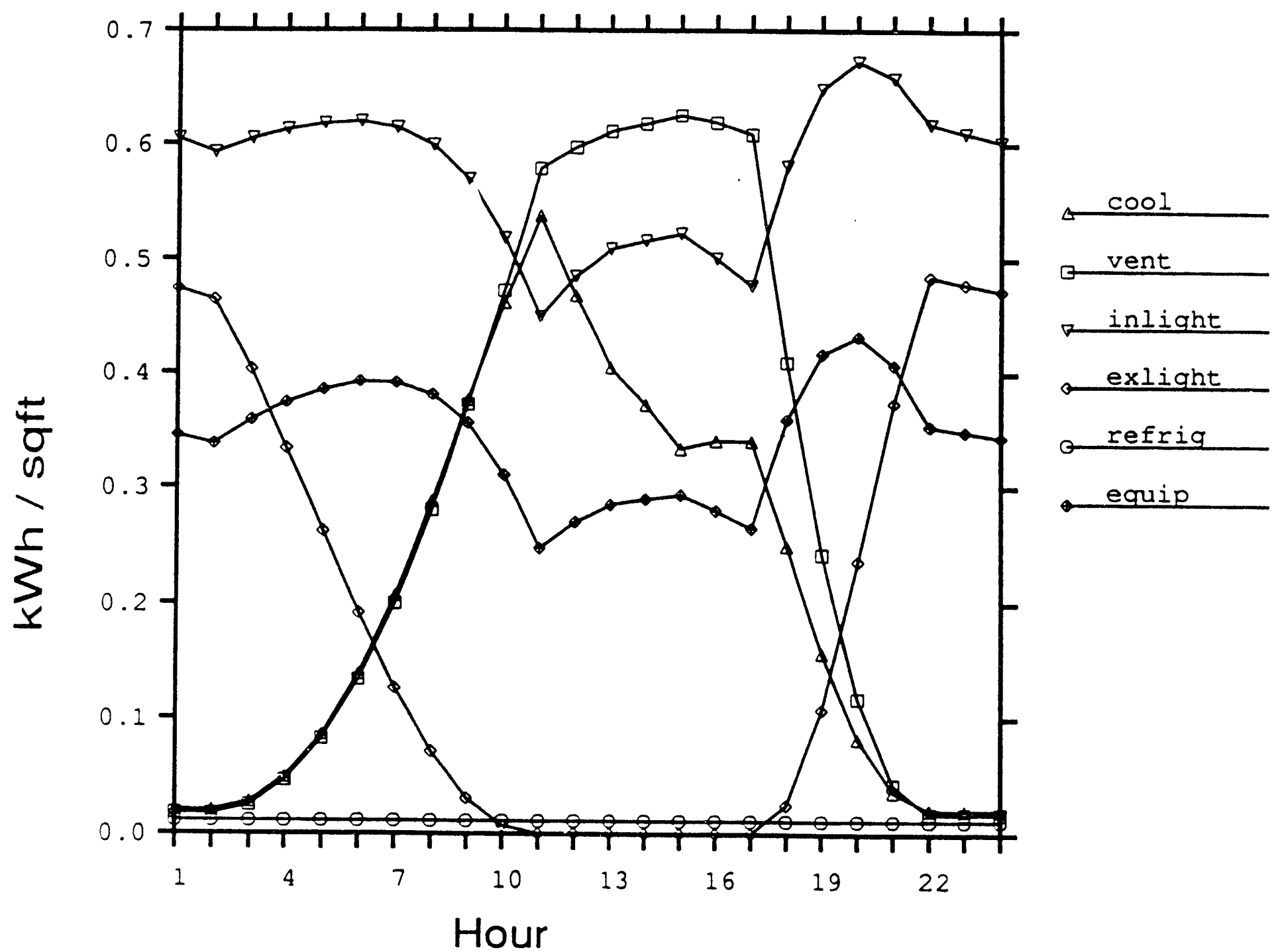


'Igret'

'StandardDayLoadShapes'

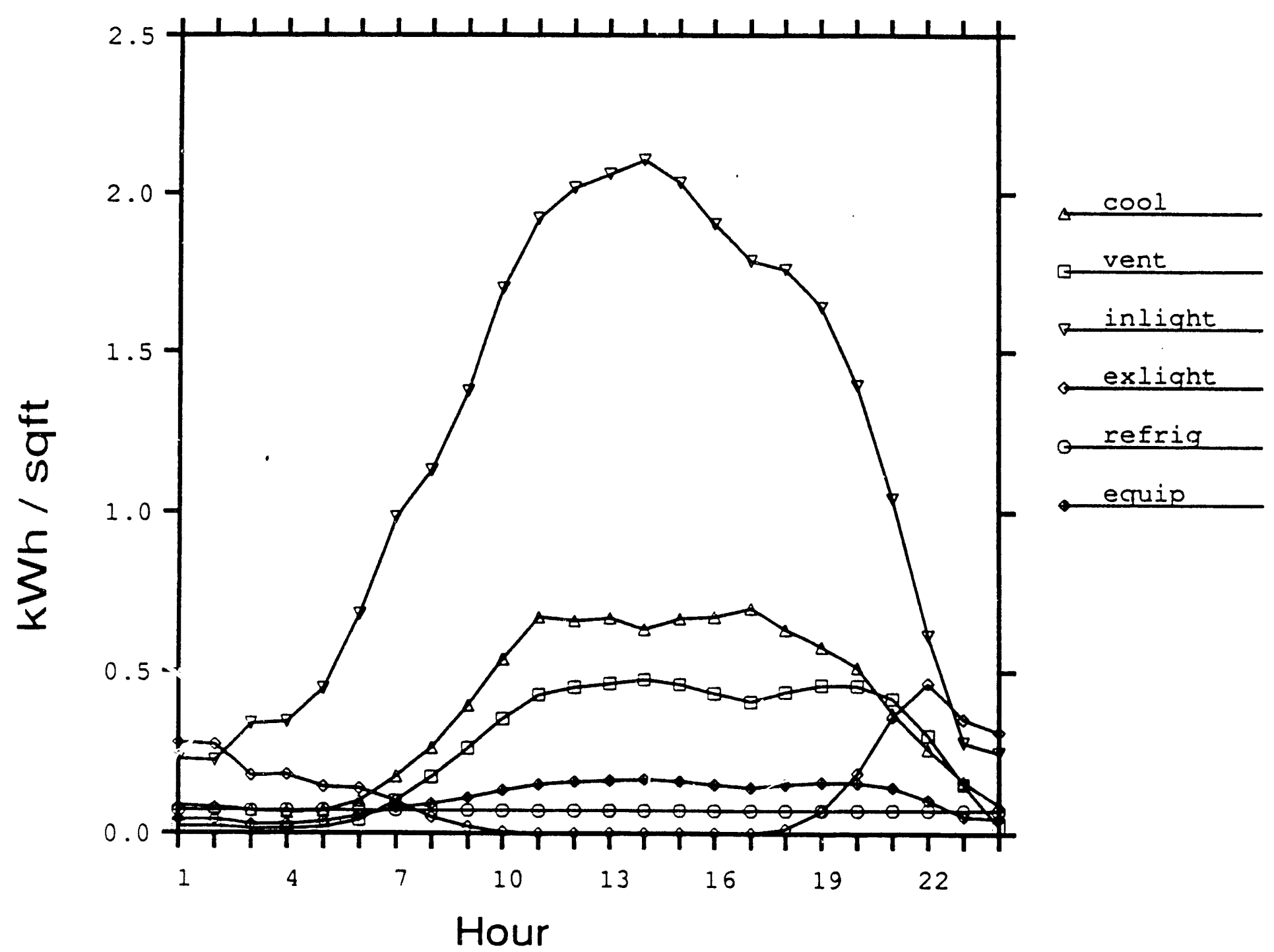




\section{'Igret'}

'NonstandardDayLoadShapes'

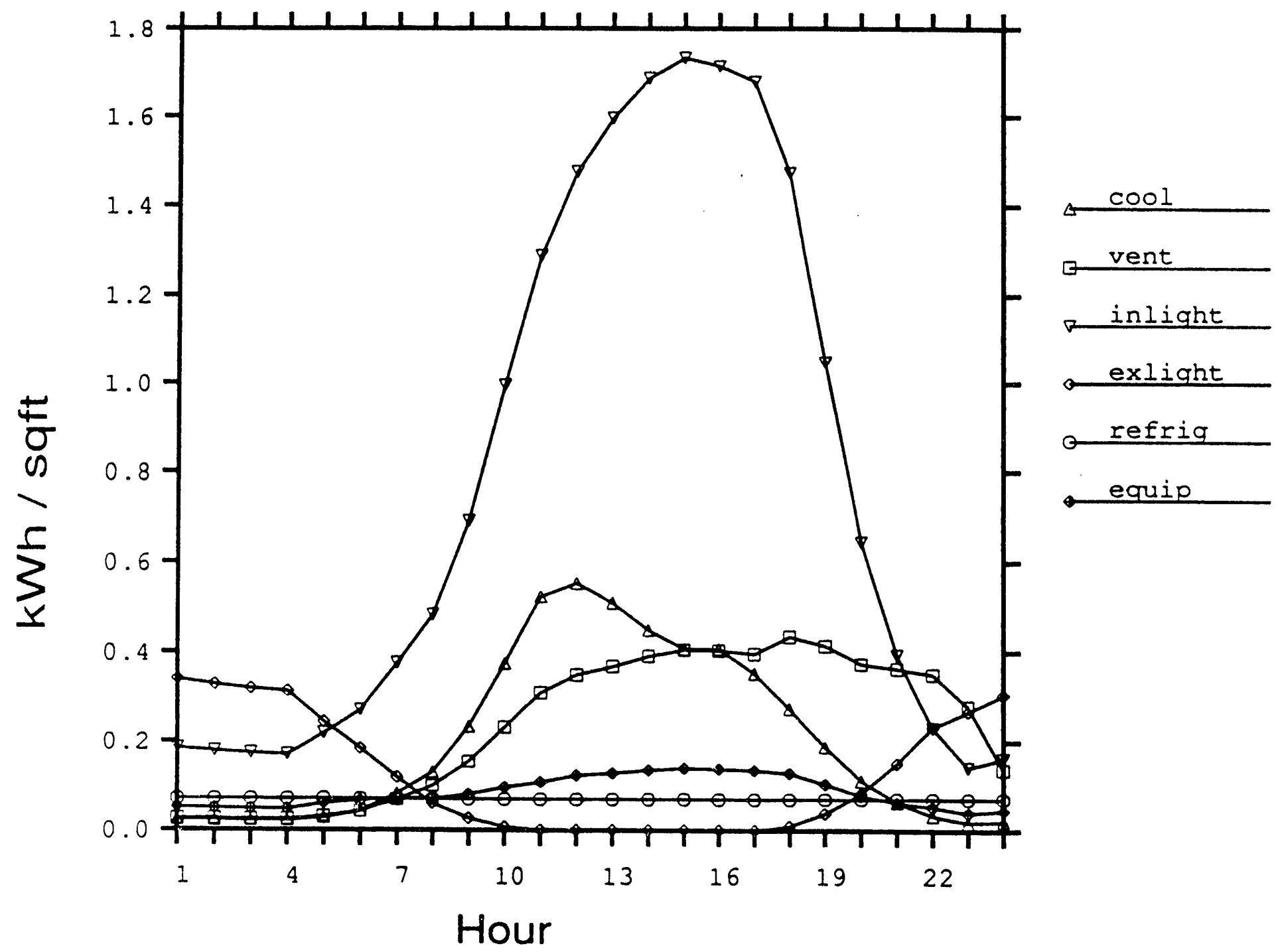




$$
\text { Fig } 49
$$

\section{'smret' \\ 'StandardDayLoadShapes'}

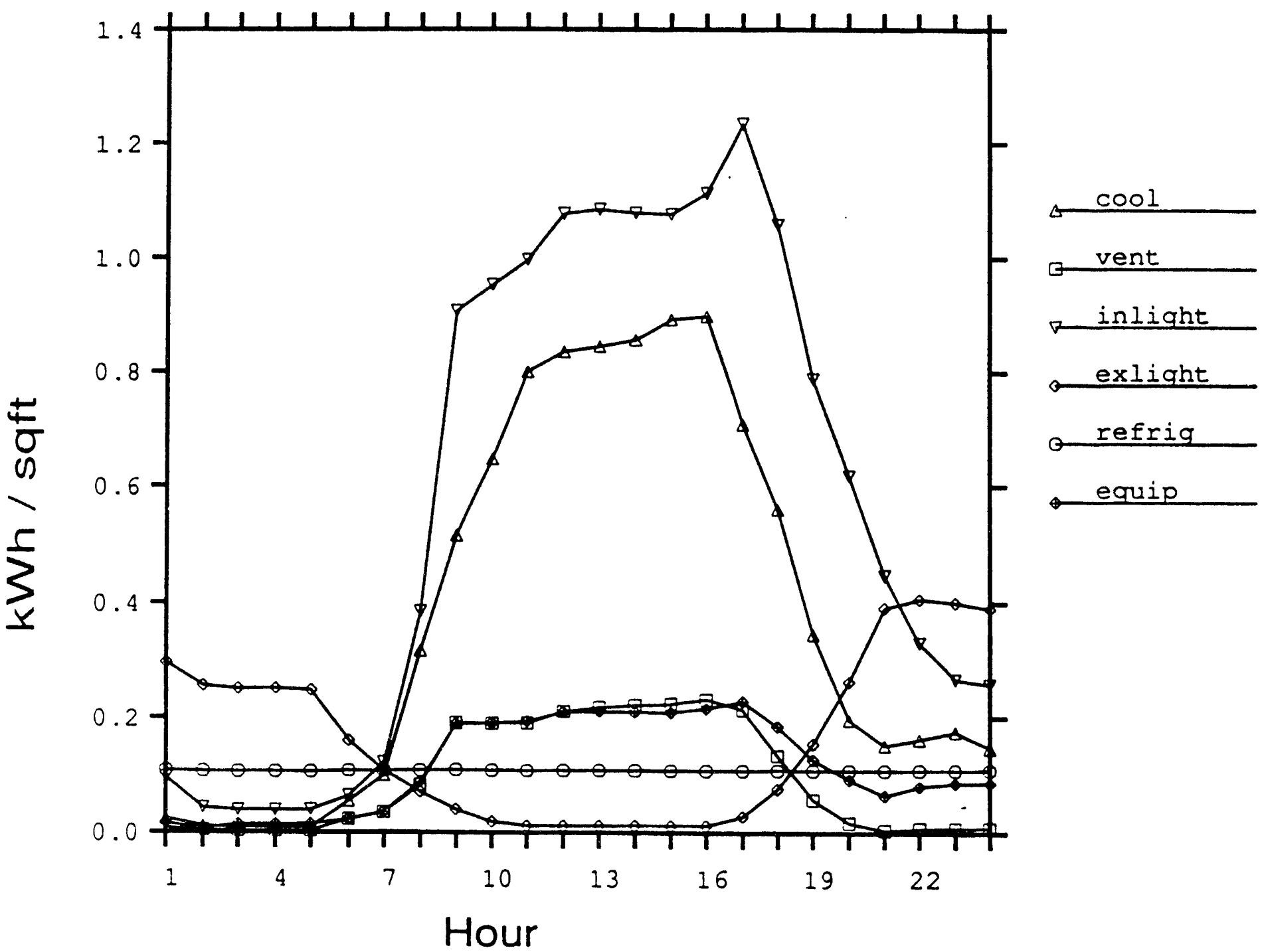




\section{'smret'}

'NonstandardDayLoadShapes'

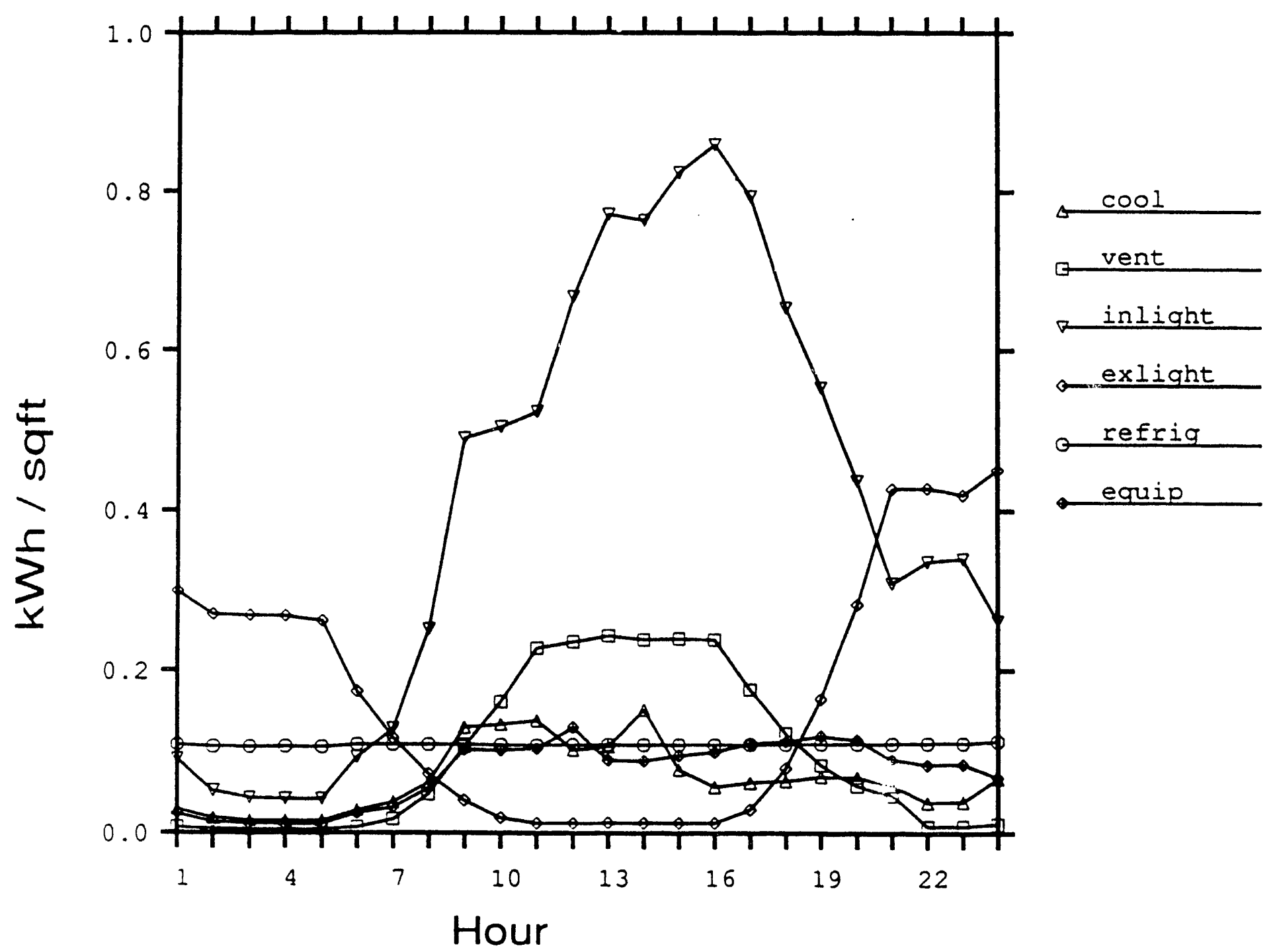




\section{Guide to the Replacement Tables for LBL-27512 rev.}

The following pages contain revised tables for the Phase I report, LBL-27512 rev. These tables are intended to be wholly substituted for those in the Phase I report. That is the table numbering corresponds exactly to that used in the Phase I report.

There are four replacement tables:

1. Table VIII-30 contains new reconciled cooling EUIs for large office, small retail, and large retail (based on the re-analysis reported in this Appendix). In addition, all U75 values have been re-expressed in $\mathrm{kBtu} / \mathrm{sqft}$.

2. Table VIII-31 contains new values for non-electric heating U75s for food store, non-refrigerated warehouse, and restaurant. This corrects a calculation error in the Pcontainse I report.

3. Table VIII-32 contains new reconciled ventilation EUls for large office, small retail, and large retail (based on the re-analysis reported in this Appendix).

4. Table VIII-33 contains new reconciled indoor lighting, outdoor lighting, miscellaneous equipment, refrigeration, cooking, and water heating EUIs for large office, small retail, and large retail (based on the re-analysis reported in this Appendix). 
Table VIII-30. Cooling EUIs for 1975 and 1980 Vintages (changes from original in boldface)

\begin{tabular}{|c|c|c|c|c|c|c|}
\hline & $\begin{array}{c}\text { Recon } \\
\text { EUI }\end{array}$ & $\begin{array}{r}\text { Load }_{\text {Proto }} \\
\text { EUI }_{\text {Proto }} \\
\end{array}$ & $\begin{array}{c}\text { Load }_{1975} f^{2} \\
\text { Load }_{\text {Proto }} \\
\end{array}$ & $\begin{array}{l}\text { Fuel } \\
\text { Type }\end{array}$ & $\begin{array}{l}1975 \\
\text { EUI }^{3,4}\end{array}$ & $\begin{array}{c}\text { Load }_{1980^{5}} \\
\text { Load }_{1975} \\
\end{array}$ \\
\hline Sm Off LAX & 2.77 & 2.48 & 0.94 & $\begin{array}{l}\text { Elec. } \\
\text { Gas } \\
\text { Other }\end{array}$ & $\begin{array}{r}6.76 \\
57.01 \\
37.40\end{array}$ & 0.91 \\
\hline Sm Off BUR & 5.80 & 2.36 & 0.98 & $\begin{array}{l}\text { Elec. } \\
\text { Gas } \\
\text { Other }\end{array}$ & $\begin{array}{r}14.02 \\
118.16 \\
77.49\end{array}$ & 0.84 \\
\hline Sm Off NOR & 8.92 & 2.19 & 0.97 & $\begin{array}{l}\text { Elec. } \\
\text { Gas } \\
\text { Other }\end{array}$ & $\begin{array}{r}19.86 \\
167.36 \\
109.76\end{array}$ & 0.92 \\
\hline Lg Off LAX & 3.01 & 3.48 & 1.98 & $\begin{array}{l}\text { Elec. } \\
\text { Gas } \\
\text { Other }\end{array}$ & $\begin{array}{r}21.71 \\
182.86 \\
119.94\end{array}$ & 0.38 \\
\hline Lg Off BUR & 3.00 & 3.41 & 1.82 & $\begin{array}{l}\text { Elec. } \\
\text { Gas } \\
\text { Other }\end{array}$ & $\begin{array}{r}19.49 \\
164.15 \\
107.67\end{array}$ & 0.40 \\
\hline Lg Off NOR & 3.91 & 3.22 & 1.72 & $\begin{array}{l}\text { Elec. } \\
\text { Gas } \\
\text { Other }\end{array}$ & $\begin{array}{r}22.66 \\
190.92 \\
125.23\end{array}$ & 0.47 \\
\hline Sm Ret LAX & 3.68 & 2.51 & 0.92 & $\begin{array}{l}\text { Elec. } \\
\text { Gas } \\
\text { Other }\end{array}$ & $\begin{array}{r}8.97 \\
62.62 \\
49.14\end{array}$ & 0.97 \\
\hline Sm Ret BUR & 4.40 & 2.38 & 0.94 & $\begin{array}{l}\text { Elec. } \\
\text { Gas } \\
\text { Other }\end{array}$ & $\begin{array}{l}10.39 \\
72.54 \\
56.93\end{array}$ & 0.87 \\
\hline Sm Ret NOR & 7.57 & 2.21 & 0.95 & $\begin{array}{l}\text { Elec. } \\
\text { Gas } \\
\text { Other }\end{array}$ & $\begin{array}{r}16.77 \\
117.12 \\
91.91\end{array}$ & 0.90 \\
\hline Lg Ret LAX & 4.52 & 2.80 & 1.17 & $\begin{array}{l}\text { Elec. } \\
\text { Gas } \\
\text { Other }\end{array}$ & $\begin{array}{r}15.63 \\
109.12 \\
85.63\end{array}$ & 0.75 \\
\hline Lg Ret BUR & 3.86 & 2.72 & 1.18 & $\begin{array}{l}\text { Elec. } \\
\text { Gas } \\
\text { Other }\end{array}$ & $\begin{array}{l}13.07 \\
91.30 \\
71.65\end{array}$ & 0.72 \\
\hline Lg Ret NOR & 5.97 & 2.59 & 1.13 & $\begin{array}{l}\text { Elec. } \\
\text { Gas } \\
\text { Other }\end{array}$ & \begin{tabular}{r|}
18.44 \\
128.76 \\
101.04
\end{tabular} & 0.84 \\
\hline
\end{tabular}


Table VIII-30. Cooling EUIs cont. ${ }^{6}$

(changes from original in boldface)

\begin{tabular}{|c|c|c|c|c|c|c|}
\hline & $\begin{array}{c}\text { Recon } \\
\text { EUI }\end{array}$ & $\begin{array}{c}\text { Load }_{\text {Protol }}{ }^{1} \\
\text { EUI }_{\text {Proto }} \\
\end{array}$ & $\begin{array}{l}\operatorname{Load}_{1975} I^{2} \\
\text { Load }_{\text {Proto }}\end{array}$ & $\begin{array}{l}\text { Fuel } \\
\text { Type }\end{array}$ & $\begin{array}{r}1975 \\
\text { EUI }^{3,4}\end{array}$ & $\begin{array}{c}\text { Load }_{1980^{\prime}} \\
\text { Load }_{1975}\end{array}$ \\
\hline \multirow{2}{*}{$\begin{array}{l}\text { NonRef Ware } \\
\text { LAX }\end{array}$} & \multirow[t]{7}{*}{1.16} & \multirow[t]{7}{*}{2.56} & 0.855 & & & \\
\hline & & & & $\begin{array}{l}\text { Elec. } \\
\text { Gas } \\
\text { Other }\end{array}$ & $\begin{array}{r}2.87 \\
18.19 \\
13.96\end{array}$ & 0.82 \\
\hline \multirow[t]{3}{*}{ BUR } & & & \multirow[t]{3}{*}{1.01} & Elec. & 3.04 & \multirow[t]{3}{*}{0.80} \\
\hline & & & & & 19.24 & \\
\hline & & & & Other & 14.77 & \\
\hline \multirow[t]{2}{*}{ NOR } & & & \multirow[t]{2}{*}{1.04} & Elec. & 3.07 & \multirow[t]{2}{*}{0.67} \\
\hline & & & & Gas & $\begin{array}{l}19.65 \\
1508\end{array}$ & \\
\hline \multirow{3}{*}{$\begin{array}{l}\text { Ref Ware } \\
\text { LAX }\end{array}$} & \multirow[t]{3}{*}{2.82} & \multirow[t]{3}{*}{2.27} & 0.97 & & & \multirow{3}{*}{0.73} \\
\hline & & & 0.79 & $\begin{array}{l}\text { Elec. } \\
\text { Gas }\end{array}$ & $\begin{array}{r}5.77 \\
36.71\end{array}$ & \\
\hline & & & & Other & 28.18 & \\
\hline \multirow[t]{2}{*}{ BUR } & & & 0.85 & $\begin{array}{l}\text { Elec. } \\
\text { Gas }\end{array}$ & $\begin{array}{r}6.24 \\
3975\end{array}$ & \multirow[t]{2}{*}{0.80} \\
\hline & & & & Other & 30.50 & \\
\hline \multirow[t]{2}{*}{ NOR } & & & 1.19 & Elec. & $\begin{array}{r}8.73 \\
\end{array}$ & \multirow[t]{2}{*}{0.72} \\
\hline & & & & Other & 42.72 & \\
\hline \multirow{3}{*}{$\begin{array}{l}\text { Restaurant } \\
\text { LAX }\end{array}$} & 12.25 & 2.55 & 0.88 & & & \multirow{3}{*}{0.96} \\
\hline & & & 0.94 & $\begin{array}{l}\text { Elec. } \\
\text { Gas }\end{array}$ & $\begin{array}{r}24.57 \\
177.01\end{array}$ & \\
\hline & & & & Other & 149.55 & \\
\hline \multirow[t]{3}{*}{ BUR } & & & 0.94 & Elec. & 24.57 & \multirow[t]{2}{*}{0.93} \\
\hline & & & & Gas & 177.01 & \\
\hline & & & & Other & 149.55 & \multirow{3}{*}{1.04} \\
\hline \multirow[t]{2}{*}{ NOR } & & & 1.15 & Elec. & $\mathbf{3 0 . 0 3}$ & \\
\hline & & & & $\begin{array}{l}\text { Gas } \\
\text { Other }\end{array}$ & $\begin{array}{l}216.32 \\
182.78\end{array}$ & \\
\hline
\end{tabular}




\section{Notes for Table VIII-30}

1. Developed from DOE-2 simulations of the NELDIG prototype.

2. Developed using an additional simulation of the prototype modified for the 1975 vintage.

3. Calculated using CEC weighted average energy conversion efficiencies by fuel type: electricity, gas, and other.

4. Converted to $\mathrm{kBtw} / \mathrm{ft}^{2} \mathrm{yr}$ using a conversion factor of $3.412 \mathrm{kBtu} / \mathrm{kWh}$.

5. Because reconciliation was not performed separately for each climate region, climate variation is introduced by multiplying the 1975 EUI (one column to the right and above) by this ratio, which was developed using additional DOE-2 simulations of the prototype in each climate region.

6. Food stores showed no cooling load in the simulations of the NELDIG prototype; consequently, all temperature-sensitive load was assigned to refrigeration, and the cooling EUI was set to zero. 
Table VIII-31. Heating EUIs cont. ${ }^{5}$

(changes to original in boldface)

\begin{tabular}{|c|c|c|c|c|c|c|}
\hline & $\begin{array}{c}\text { NELDIG } \\
\text { EUI }\end{array}$ & $\begin{array}{l}\text { Load }_{\text {Proto }} \\
\text { EUI I }_{\text {Proto }}\end{array}$ & Load $_{\text {Proto }}{ }^{1}$ & $\begin{array}{l}\text { Fuel } \\
\text { Type }\end{array}$ & EUT & Load $_{1975}^{980}{ }^{3}$ \\
\hline $\begin{array}{l}\text { Food Store } \\
\text { LAX }^{4}\end{array}$ & 2.44 & 0.74 & $\begin{array}{l}1.17 \\
1.00\end{array}$ & $\begin{array}{l}\text { Elec. } \\
\text { Gas } \\
\text { Other }\end{array}$ & $\begin{array}{r}3.90 \\
10.93 \\
10.93\end{array}$ & 1.00 \\
\hline BUR & & & 1.00 & $\begin{array}{l}\text { Elec. } \\
\text { Gas } \\
\text { Other }\end{array}$ & $\begin{array}{r}3.90 \\
10.93 \\
10.93\end{array}$ & 1.00 \\
\hline NOR & & & 1.00 & $\begin{array}{l}\text { Elec. } \\
\text { Gas } \\
\text { Other }\end{array}$ & $\begin{array}{r}3.90 \\
10.93 \\
10.93\end{array}$ & 1.00 \\
\hline NonRef Ware & 1.48 & 0.74 & 0.91 & & & \\
\hline LAX & & & 1.00 & $\begin{array}{l}\text { Elec. } \\
\text { Gas } \\
\text { Other }\end{array}$ & $\begin{array}{l}1.02 \\
1.58 \\
1.58\end{array}$ & 1.00 \\
\hline BUR & & & 1.00 & $\begin{array}{l}\text { Elec. } \\
\text { Gas } \\
\text { Other }\end{array}$ & $\begin{array}{l}1.02 \\
1.58 \\
1.58\end{array}$ & 1.00 \\
\hline NOR & & & 1.00 & $\begin{array}{l}\text { Elec. } \\
\text { Gas } \\
\text { Other }\end{array}$ & $\begin{array}{l}1.02 \\
1.58 \\
1.58\end{array}$ & 1.00 \\
\hline $\begin{array}{l}\text { Restaurant } \\
\text { LAX }\end{array}$ & 5.47 & 0.74 & $\begin{array}{l}1.58 \\
0.99\end{array}$ & $\begin{array}{l}\text { Elec. } \\
\text { Gas } \\
\text { Other }\end{array}$ & $\begin{array}{r}6.92 \\
11.36 \\
11.36\end{array}$ & 1.02 \\
\hline BUR & & & 1.00 & $\begin{array}{l}\text { Elec. } \\
\text { Gas } \\
\text { Other }\end{array}$ & $\begin{array}{r}6.94 \\
11.39 \\
11.39\end{array}$ & 1.08 \\
\hline NOR & & & 1.01 & $\begin{array}{l}\text { Elec. } \\
\text { Gas } \\
\text { Other }\end{array}$ & $\begin{array}{r}6.98 \\
11.45 \\
11.45\end{array}$ & 1.05 \\
\hline
\end{tabular}


Table VIII-32. Ventilation EUIs for 1975 and 1980 Vintages (changes to original in boldface)

\begin{tabular}{|l|c|c|ccc|}
\hline \multicolumn{2}{|c}{$\begin{array}{c}\text { Reconciled } \\
\text { EUI }\end{array}$} & $\begin{array}{c}1975 /{ }^{1} \\
\text { Prototype }\end{array}$ & $\begin{array}{r}1975 \mathrm{ELI} \\
\left(\mathrm{kWh} / \mathrm{ft}^{2} \mathrm{yr}\right)\end{array}$ & $\begin{array}{r}1975 \mathrm{EUI} \\
\left(\mathrm{kBtu} / \mathrm{ft}^{2} \mathrm{yr}\right)\end{array}$ & $\begin{array}{c}1980 /^{2} \\
1975\end{array}$ \\
\hline Small Office LAX & 0.97 & 0.93 & 0.90 & 3.08 & 1.02 \\
Small Office BUR & 1.38 & 0.97 & 1.34 & 4.58 & 0.97 \\
Small Office NOR & 1.23 & 0.98 & 1.20 & 4.09 & 0.98 \\
Large Office LAX & $\mathbf{2 . 4 5}$ & 1.52 & $\mathbf{3 . 7 2}$ & $\mathbf{1 2 . 7 1}$ & 0.36 \\
Large Office BUR & $\mathbf{2 . 6 0}$ & 1.49 & $\mathbf{3 . 8 7}$ & $\mathbf{1 3 . 2 2}$ & 0.37 \\
Large Office NOR & $\mathbf{2 . 7 2}$ & 1.47 & $\mathbf{4 . 0 0}$ & $\mathbf{1 3 . 6 4}$ & 0.38 \\
Small Retail LAX & $\mathbf{1 . 1 7}$ & 0.92 & $\mathbf{1 . 0 8}$ & $\mathbf{3 . 6 7}$ & 1.04 \\
Small Retail BUR & $\mathbf{1 . 2 6}$ & 0.94 & $\mathbf{1 . 1 8}$ & $\mathbf{4 . 0 4}$ & 1.01 \\
Small Retail NOR & $\mathbf{1 . 4 3}$ & 0.95 & $\mathbf{1 . 3 6}$ & $\mathbf{4 . 6 4}$ & 1.02 \\
Large Retail LAX & $\mathbf{2 . 6 5}$ & 1.05 & $\mathbf{2 . 7 8}$ & $\mathbf{9 . 4 9}$ & 0.97 \\
Large Retail BUR & $\mathbf{2 . 8 8}$ & 1.05 & $\mathbf{3 . 0 2}$ & $\mathbf{1 0 . 3 2}$ & 0.96 \\
Large Retail NOR & $\mathbf{2 . 8 1}$ & 1.05 & $\mathbf{2 . 9 5}$ & $\mathbf{1 0 . 0 7}$ & 0.96 \\
\hline
\end{tabular}

1. Based on DOE-2 simulations of the NELDIG prototype and a prototype modified for the 1975 vintage.

2. Based on DOE-2 simulations of the 1975 vintage prototype and a prototype modified for the 1980 vintage. 
Table VIII-33. Electric Non-HVAC EUIs for 1975 Vintage

(changes to original in boldface)

\begin{tabular}{|c|c|c|c|c|c|c|}
\hline & In Light & Ex Light & Misc Eq & Refrig & Cook & Water Ht \\
\hline \multicolumn{7}{|c|}{ Reconciled EUIs $\left(\mathrm{kWh} / \mathrm{ft}^{2}\right)$} \\
\hline Small Office & 5.47 & 1.24 & 3.59 & 0.23 & 0.04 & 0.12 \\
\hline Large Office & 9.05 & 1.61 & 3.21 & 0.10 & 0.00 & 0.12 \\
\hline Small Retail & 4.71 & 1.36 & 0.91 & 0.94 & 0.01 & 0.03 \\
\hline Large Retail & 9.44 & 1.14 & 0.87 & 0.61 & 0.17 & 0.02 \\
\hline Food Store & 11.96 & 2.01 & 1.77 & 23.17 & 0.24 & 0.03 \\
\hline Ref Ware & 3.02 & 0.55 & 6.24 & 11.34 & 0.01 & 0.17 \\
\hline NonRef Ware & 3.38 & 0.17 & 0.70 & 0.41 & 0.00 & 0.03 \\
\hline Restaurant & 7.94 & 4.09 & 4.89 & 10.78 & 4.46 & 0.03 \\
\hline \multicolumn{7}{|c|}{ Saturation from on-site survey and sampling weights $(\%)^{1}$} \\
\hline Small Office & 1.00 & 1.00 & 1.00 & 0.77 & 0.16 & 0.42 \\
\hline Large Office & 1.00 & 1.00 & 1.00 & 0.81 & 0.43 & 0.28 \\
\hline Small Retail & 1.00 & 1.00 & 1.00 & 0.79 & 0.10 & 0.26 \\
\hline Large Retail & 1.00 & 1.00 & 1.00 & 0.99 & 0.56 & 0.14 \\
\hline Food Store & 1.00 & 1.00 & 1.00 & 1.00 & 0.27 & 0.13 \\
\hline Ref Ware & 1.00 & 1.00 & 1.00 & 1.00 & 0.66 & 0.73 \\
\hline NonRef Ware & 1.00 & 1.00 & 1.00 & 0.83 & 0.00 & 0.76 \\
\hline Restaurant & 1.00 & 1.00 & 1.00 & 1.00 & 0.90 & 0.02 \\
\hline \multicolumn{7}{|c|}{1975 EUIs $\left(\mathrm{kBtu} / \mathrm{ft}^{2}\right)^{2}$} \\
\hline Small Office & 19.95 & 4.51 & 7.84 & 1.10 & 1.03 & 1.05 \\
\hline Large Office & 33.04 & 5.88 & 7.01 & 0.45 & 0.00 & 1.56 \\
\hline Small Retail & 17.20 & 4.97 & 2.51 & .4 .34 & 0.37 & 0.42 \\
\hline Large Retail & 34.46 & 4.16 & 2.40 & 2.25 & 1.11 & 0.52 \\
\hline Food Store & 43.66 & 7.34 & 4.86 & 84.59 & 3.27 & 0.84 \\
\hline Ref Ware & 11.03 & 2.01 & 17.14 & 41.40 & 0.06 & 0.86 \\
\hline NonRef Ware & 12.34 & 0.62 & 1.92 & 1.81 & 0.00 & 0.14 \\
\hline Restaurant & 28.99 & 14.93 & 13.43 & 39.36 & 18.11 & 6.44 \\
\hline
\end{tabular}

1. Developed on a total floor area basis using the on-site survey and the same weighting factors used for prototype development.

2. Developed using CEC short-run price elasticities and SCE historic prices and, for miscellaneous equipment only, a CEC technology growth adjustment factor. For all end uses but miscellaneous equipment, the price effect increases the reconciled EUIs by $7 \%$. For office miscellaneous equipment, the combined effect reduces the miscellaneous equipment EUI by $36 \%$; for all other building types, the combined effect reduces the miscellaneous equipment EUI by $19 \%$. 
Appendix B. DOE-2 BDL Prototype Input Files 

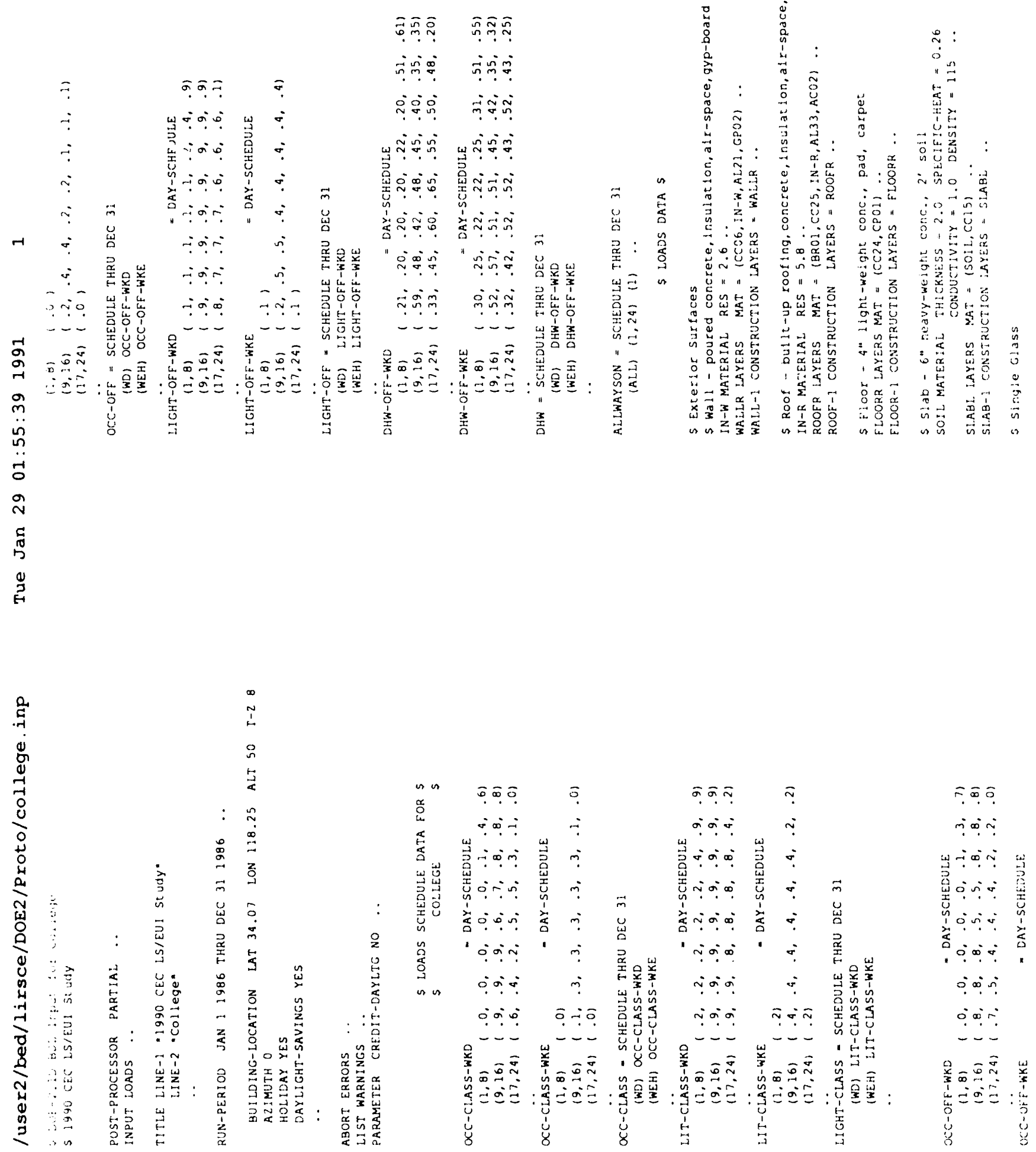


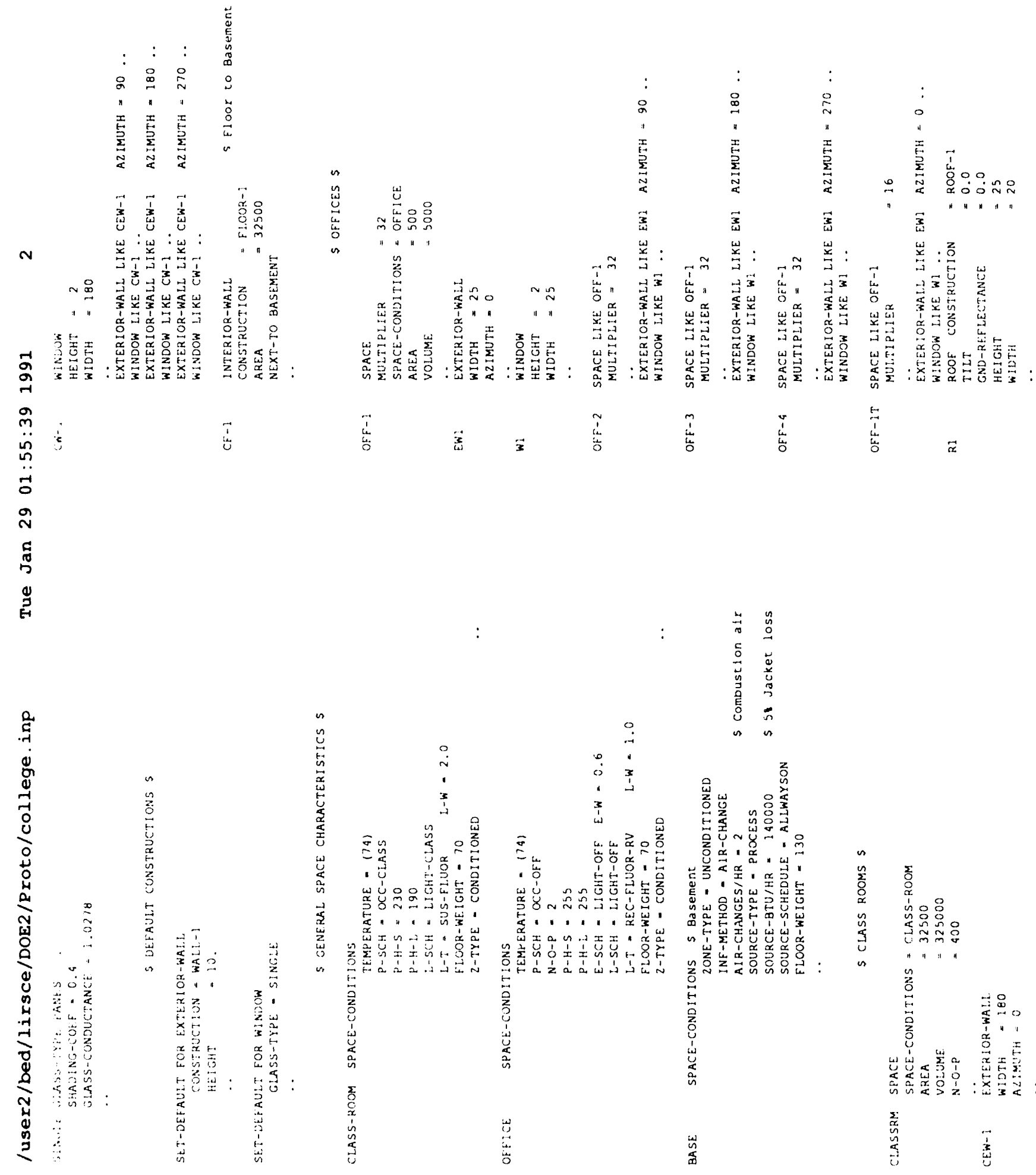



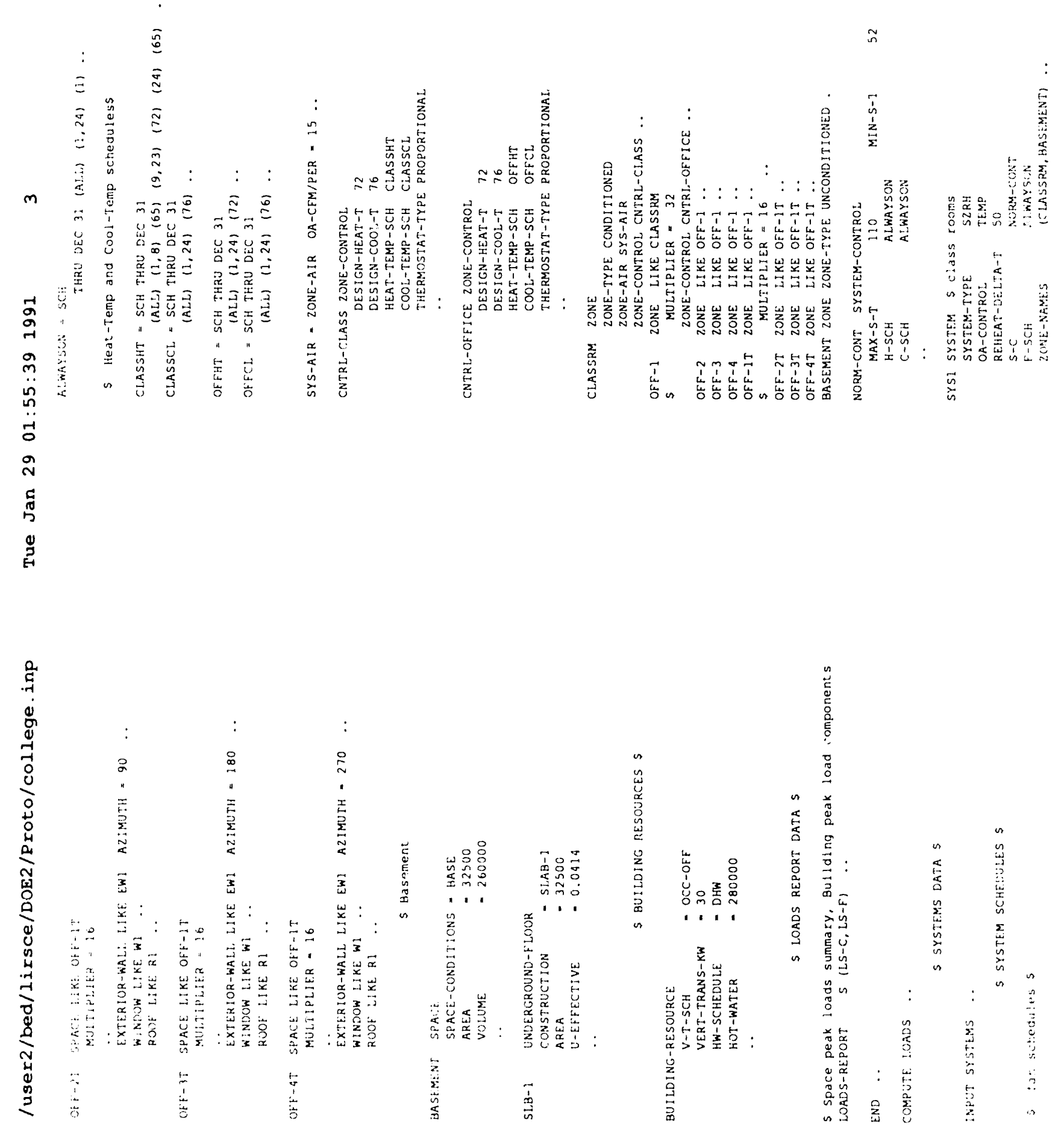

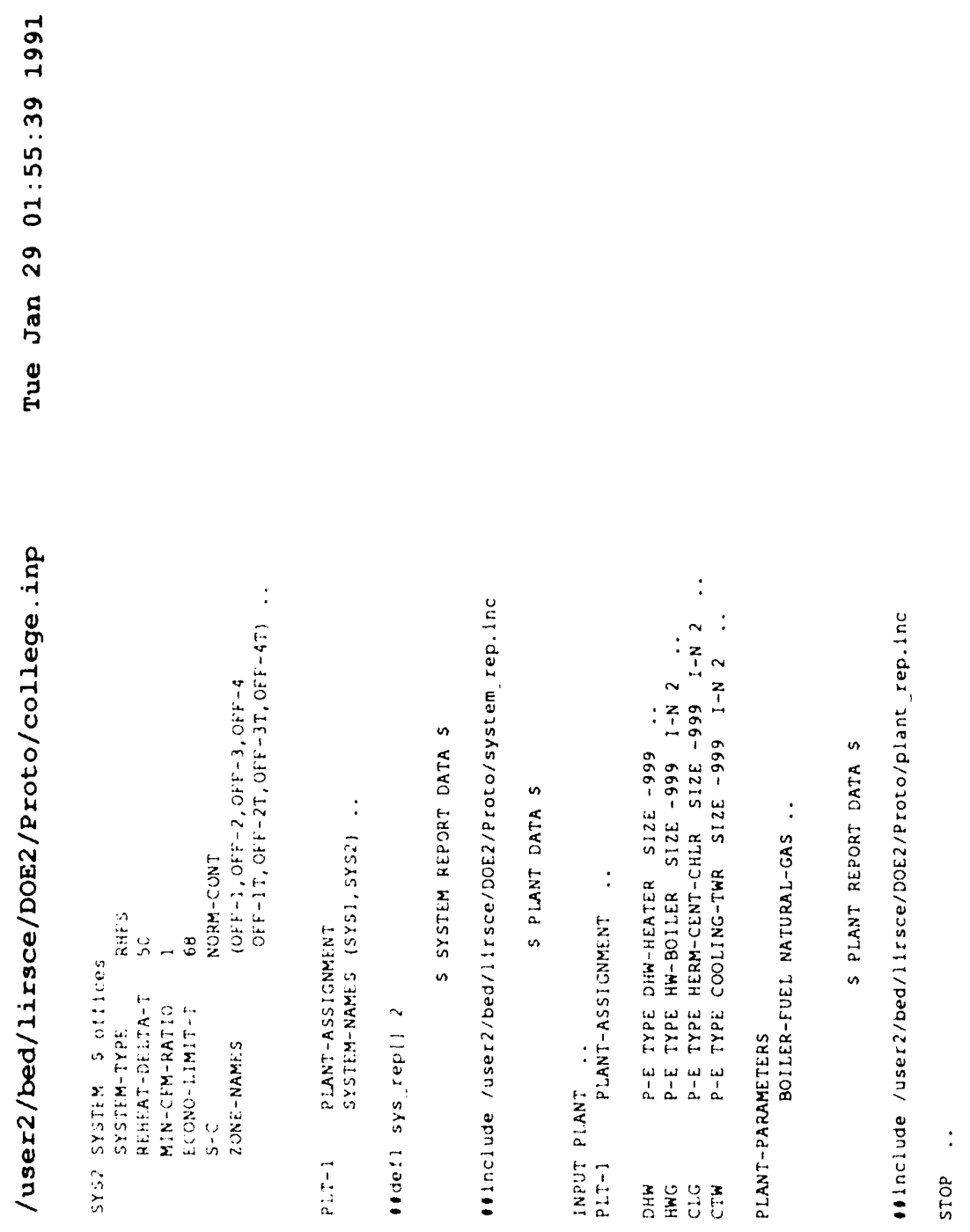

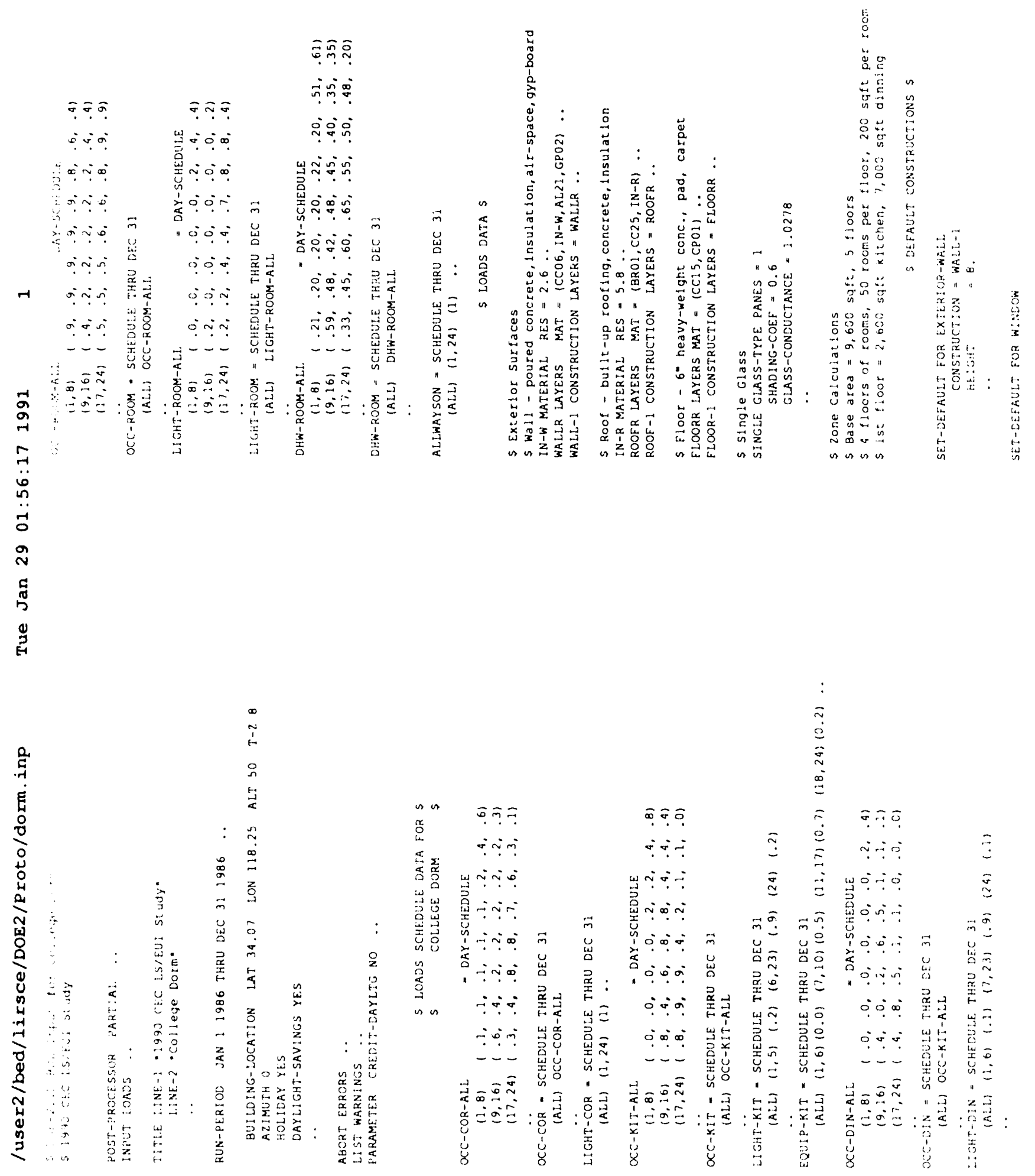


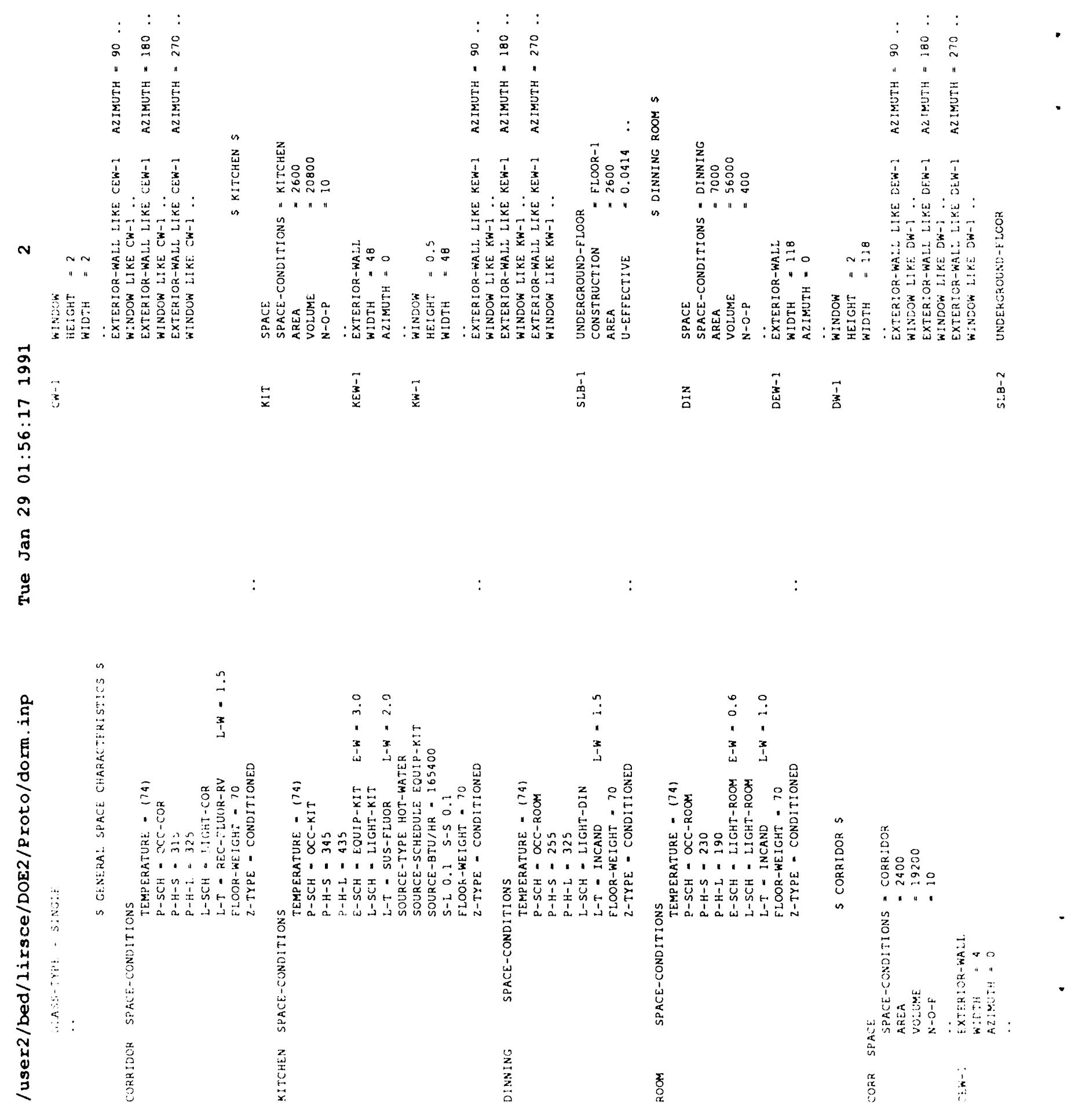



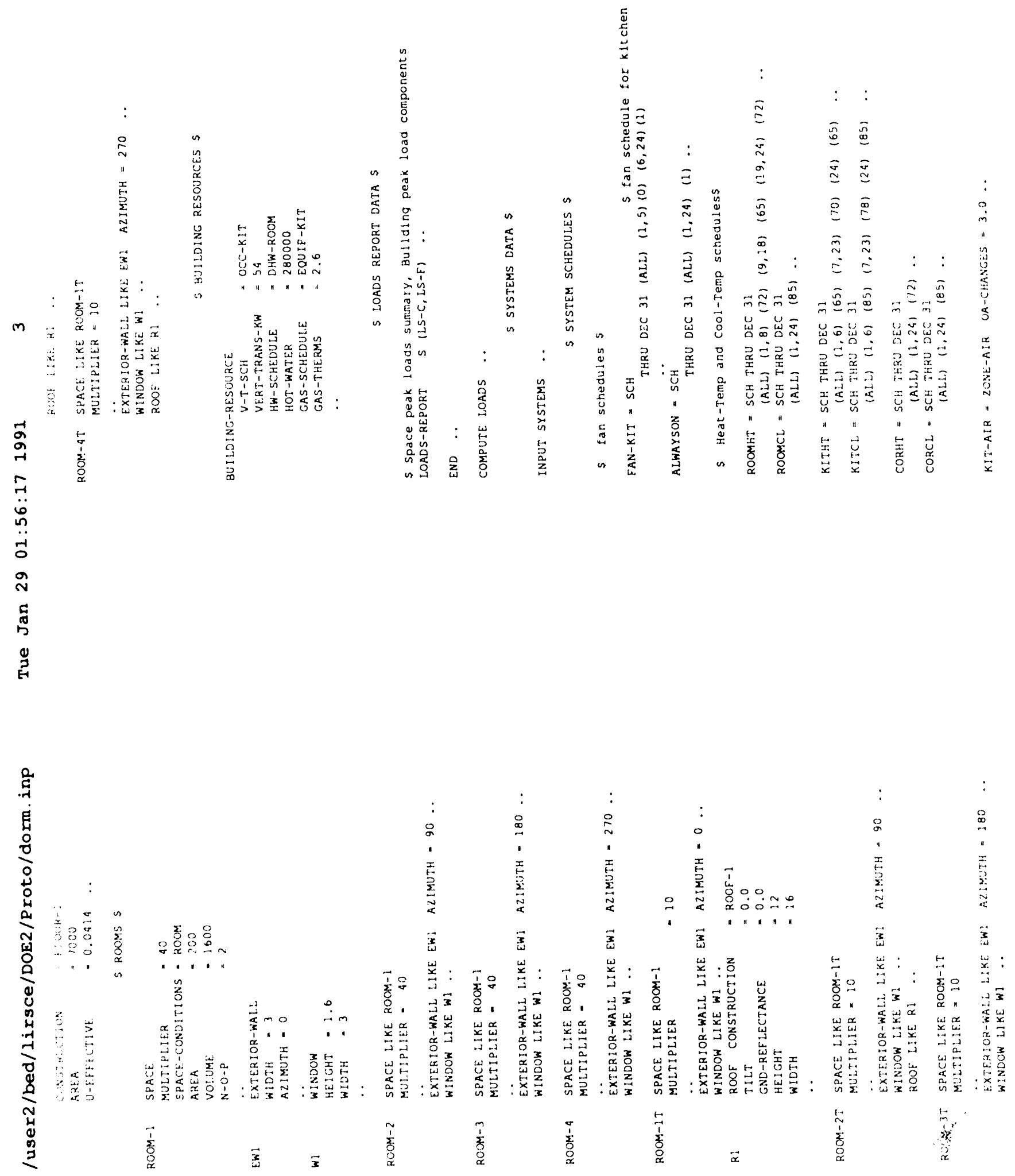

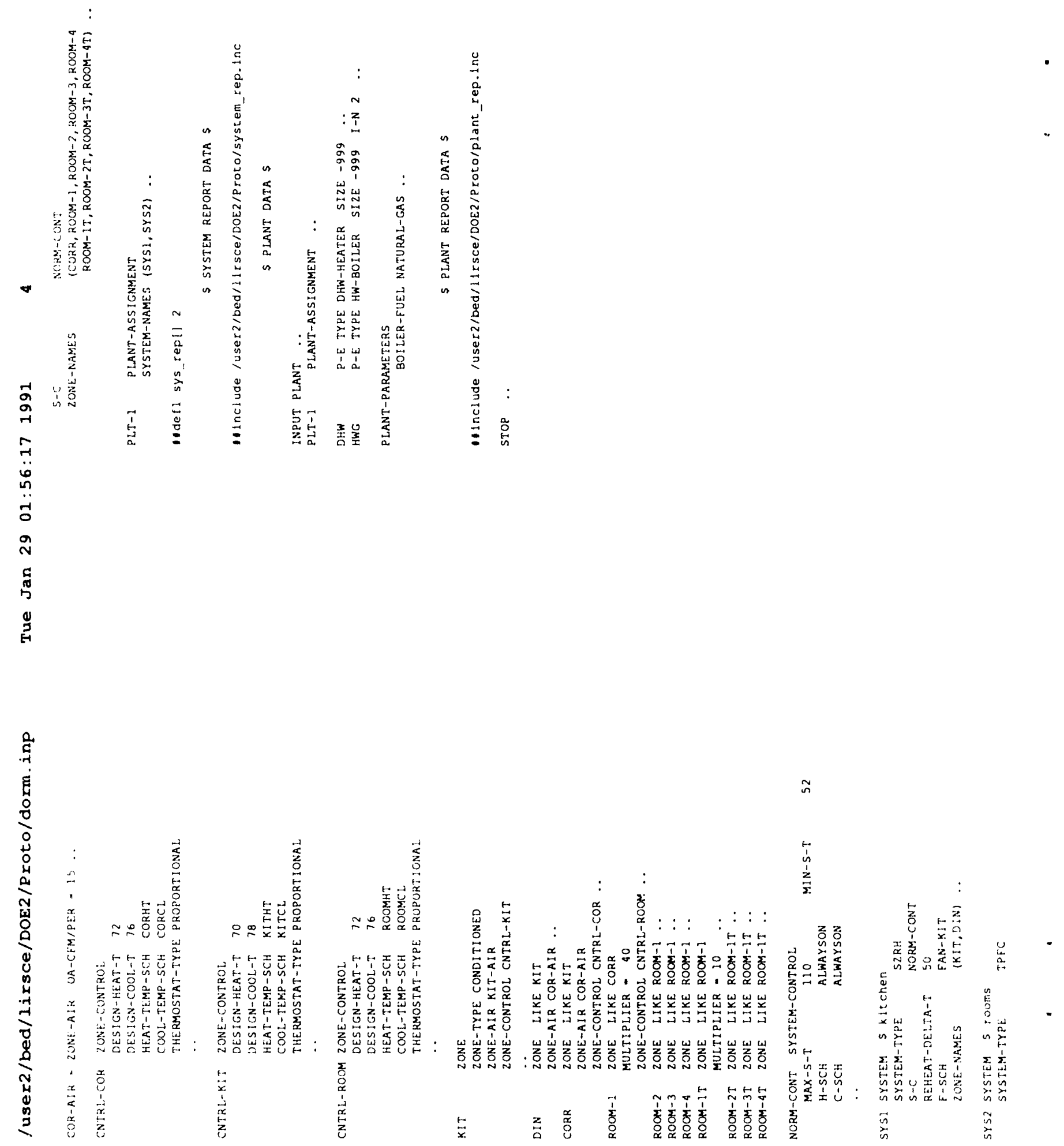

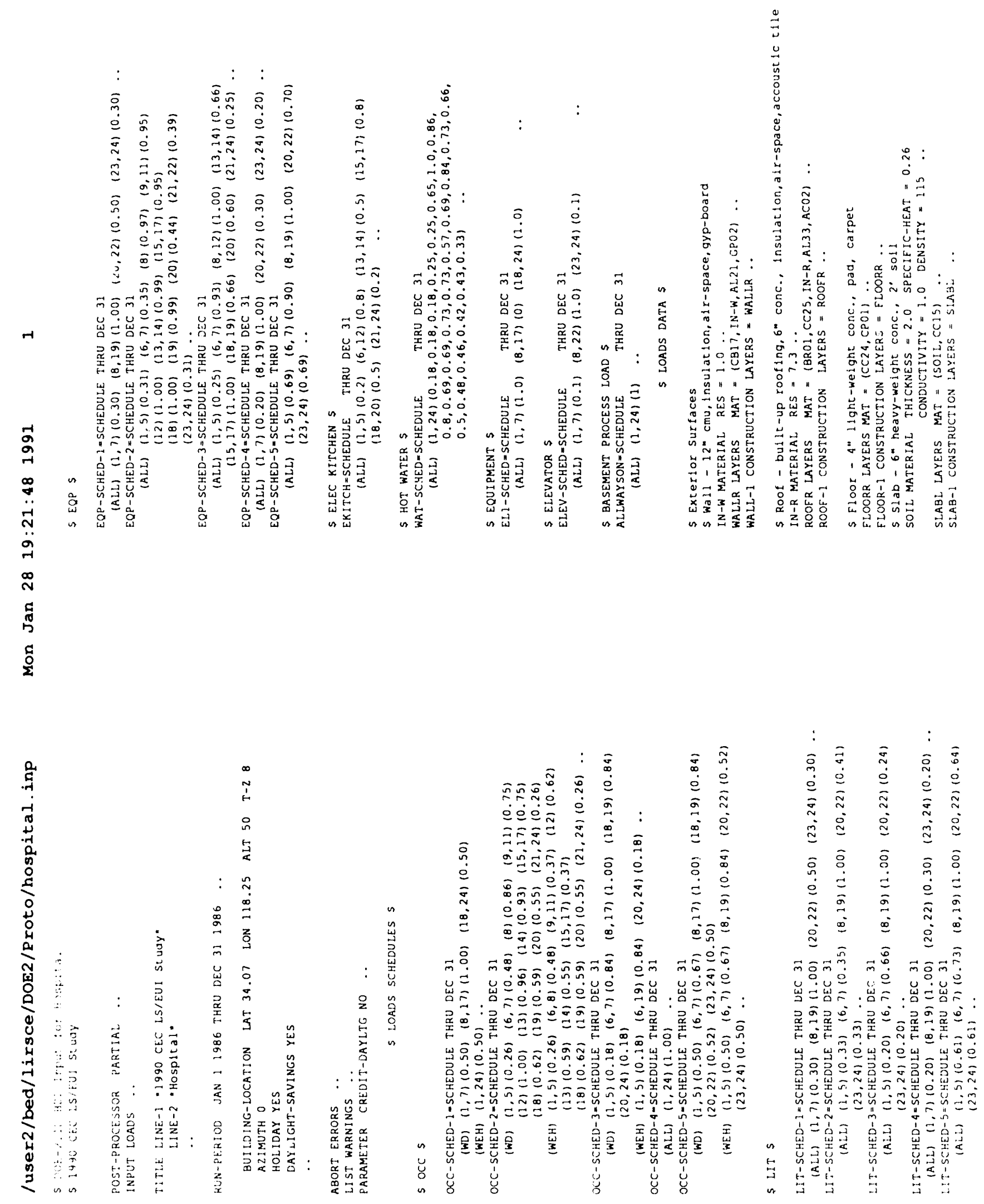

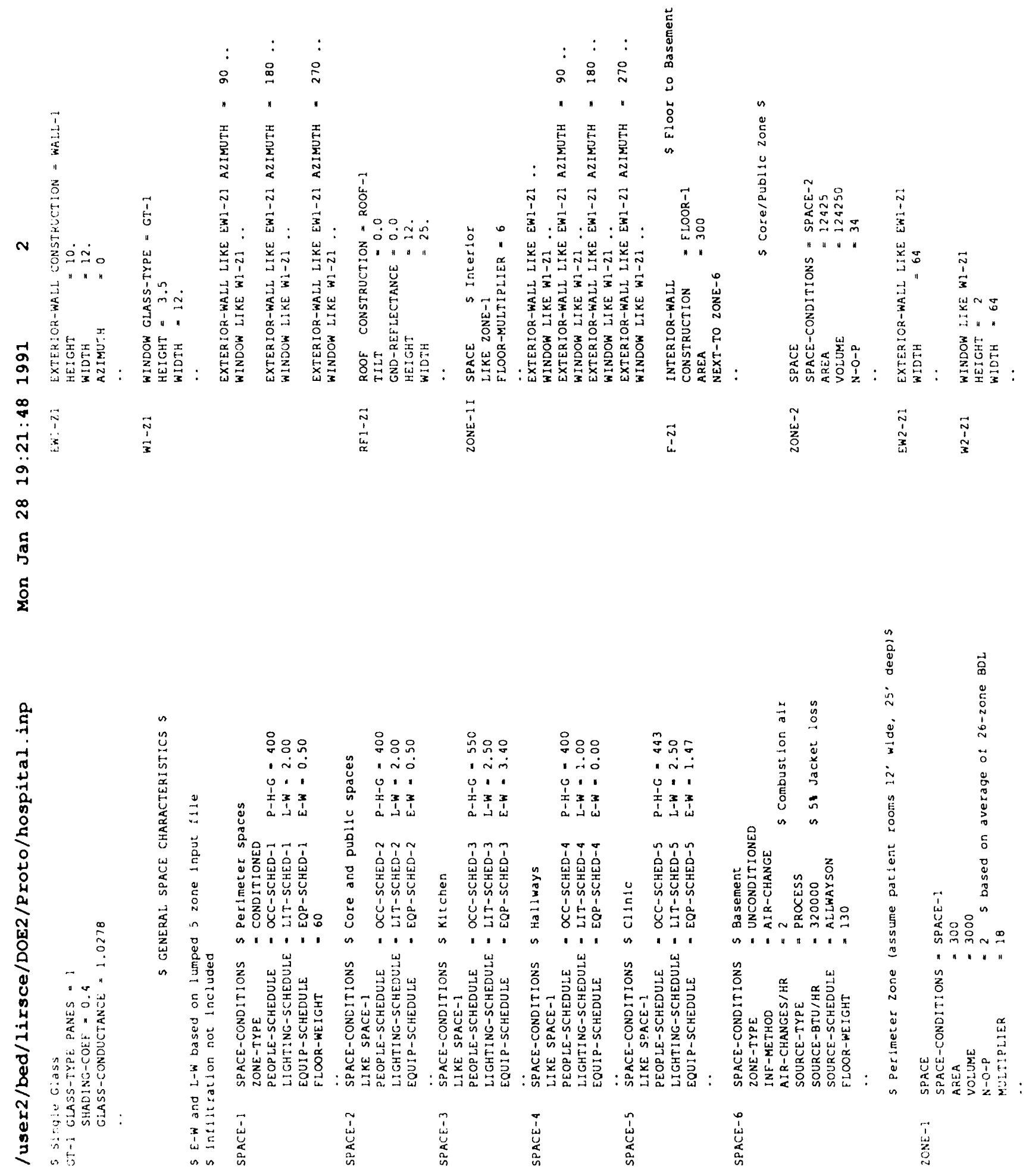

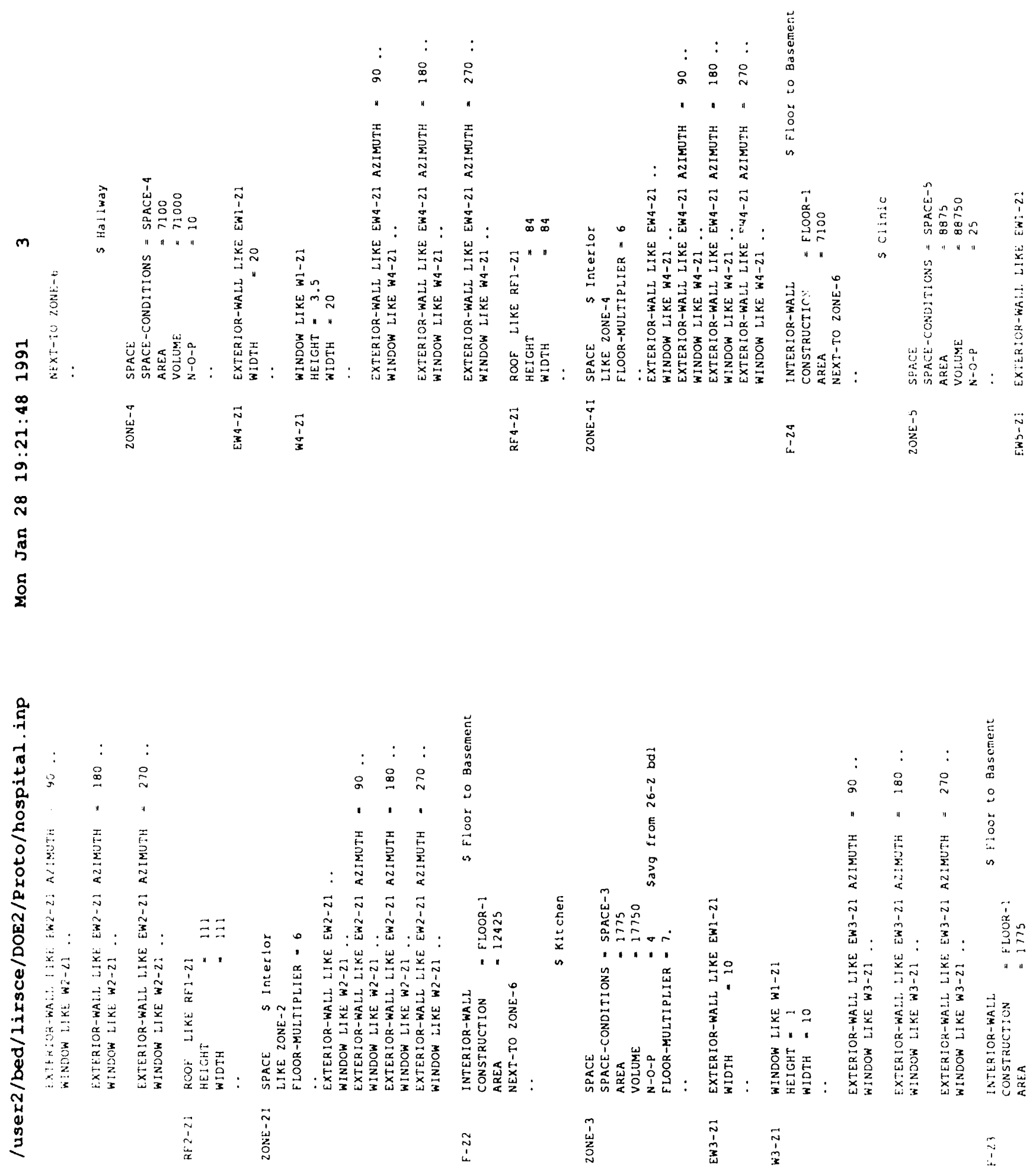


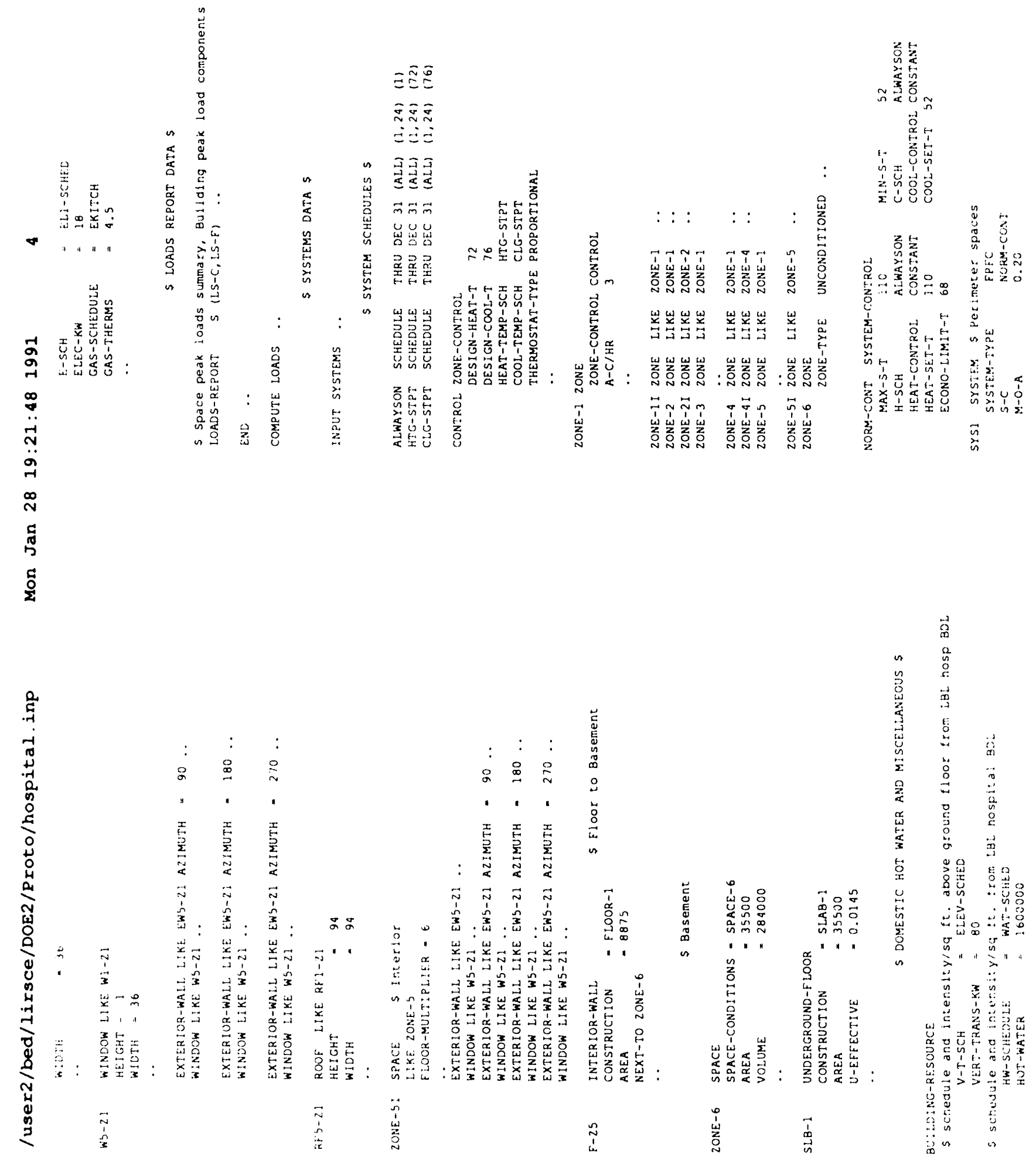



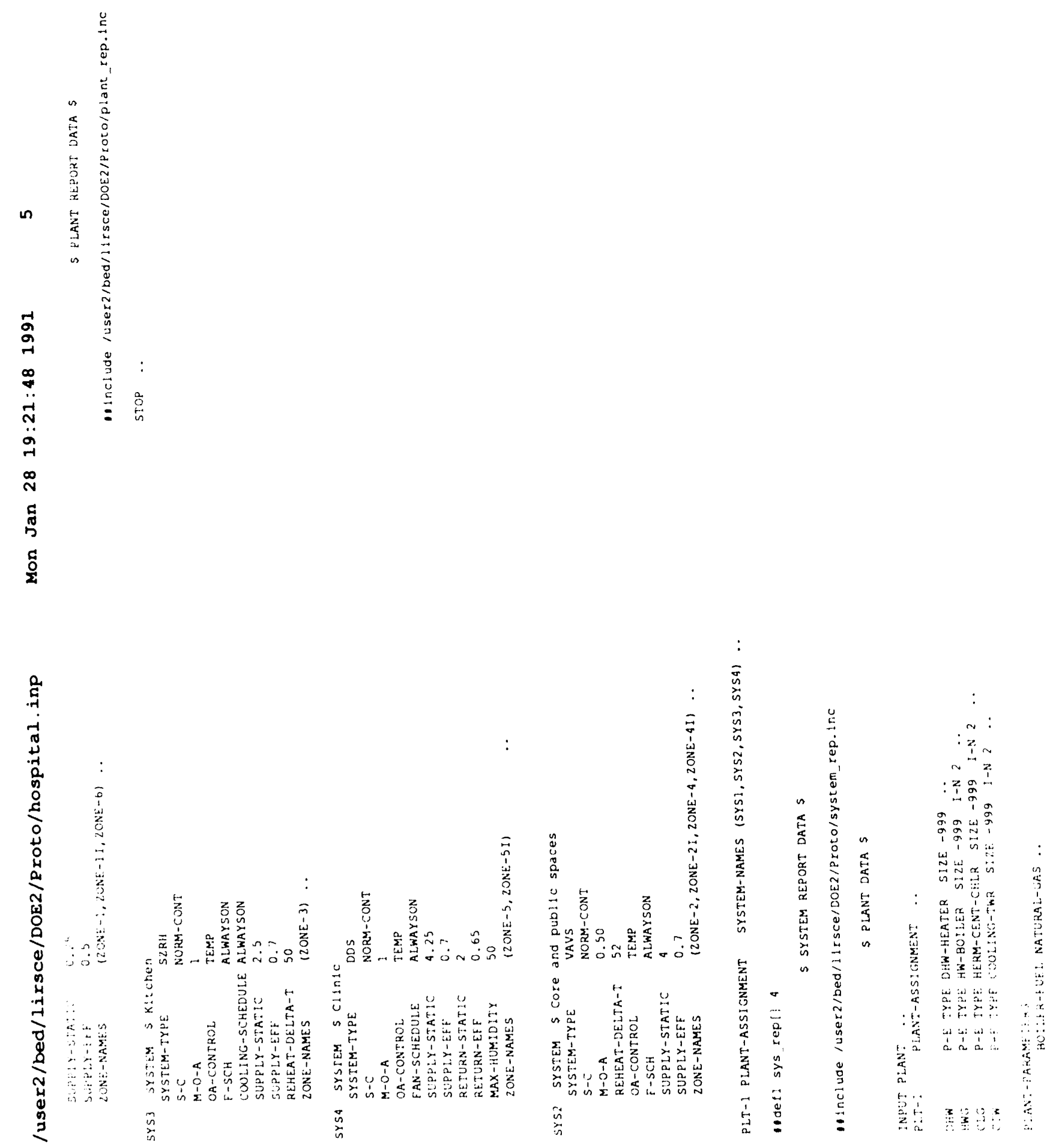

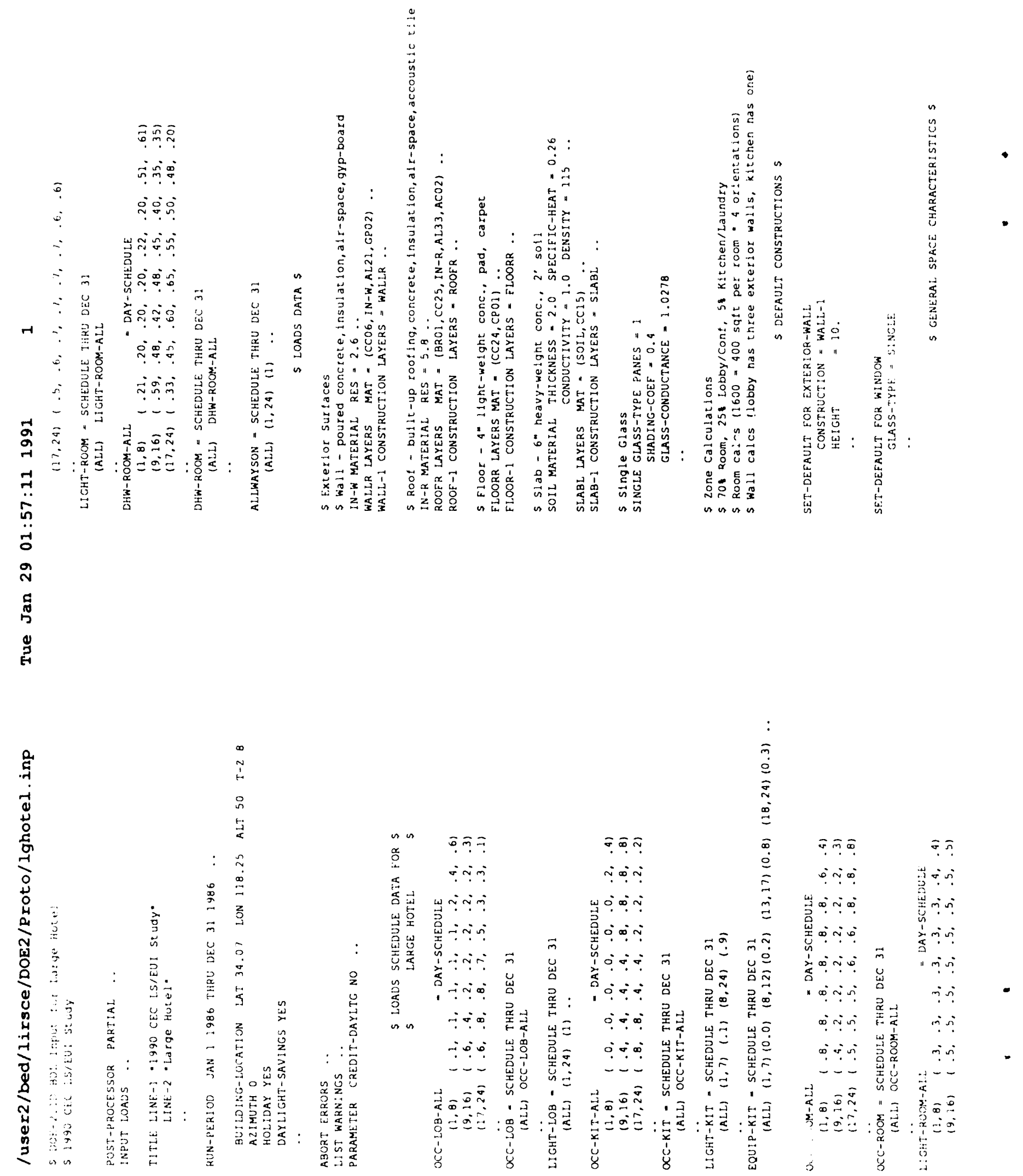


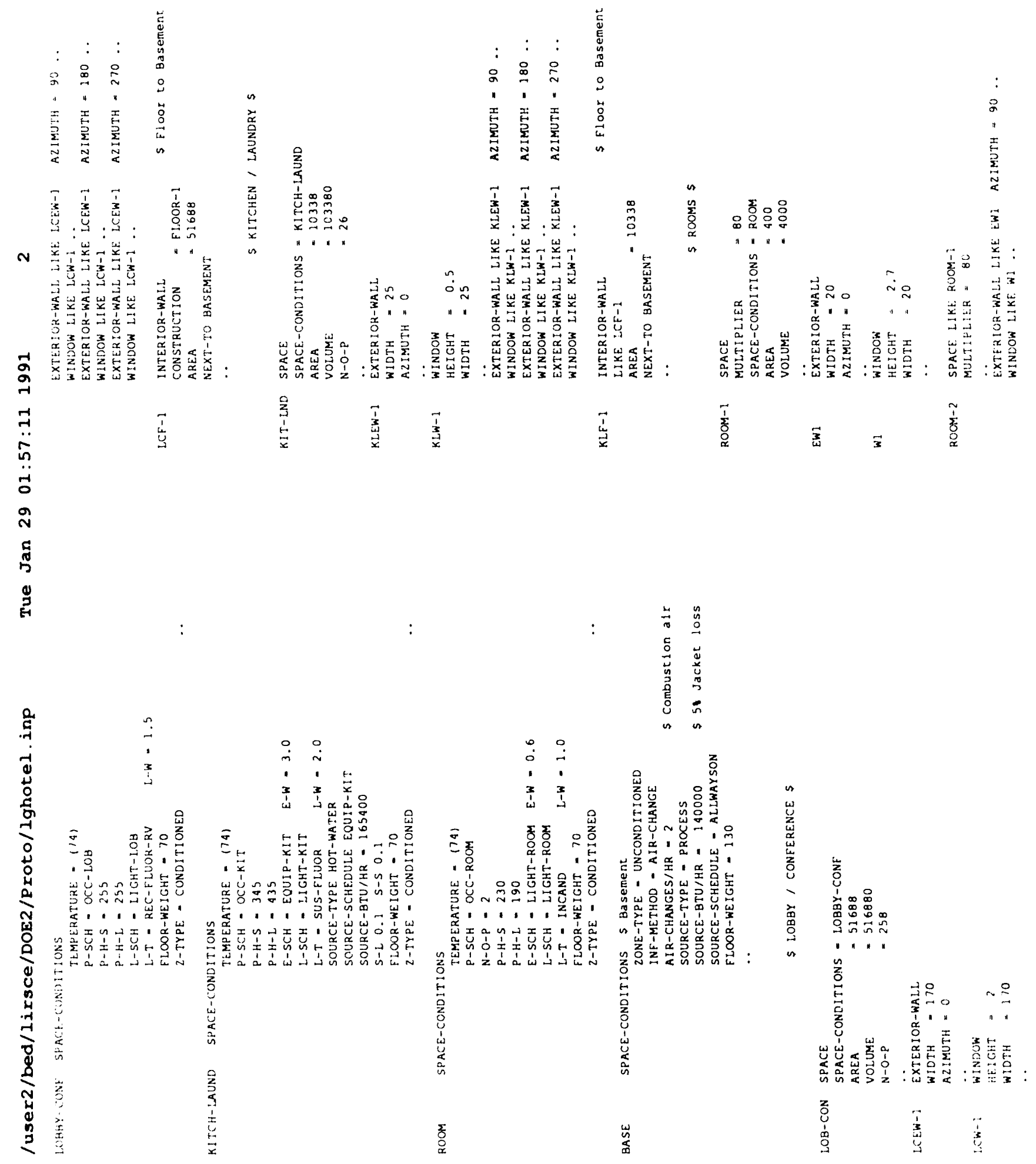



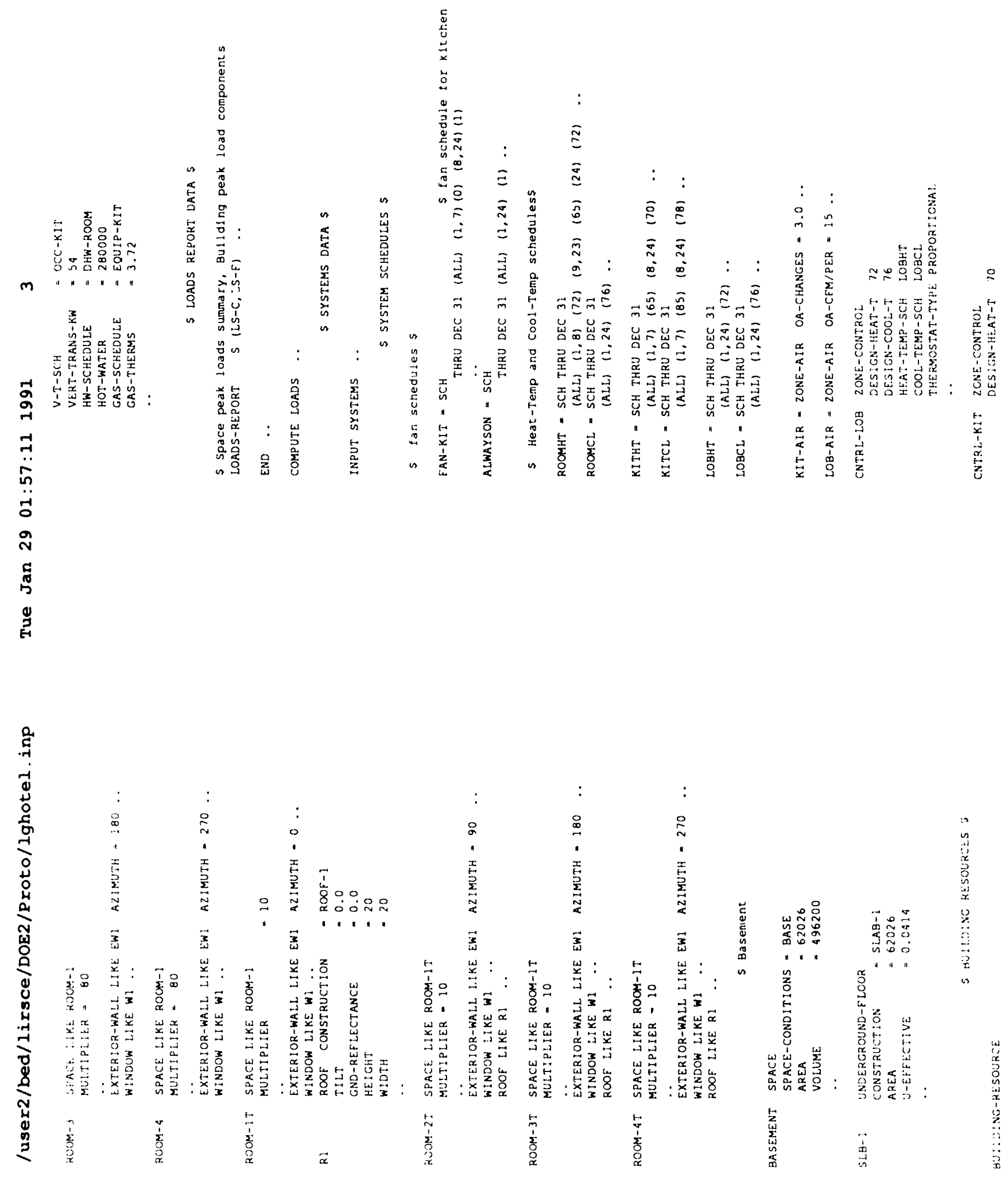

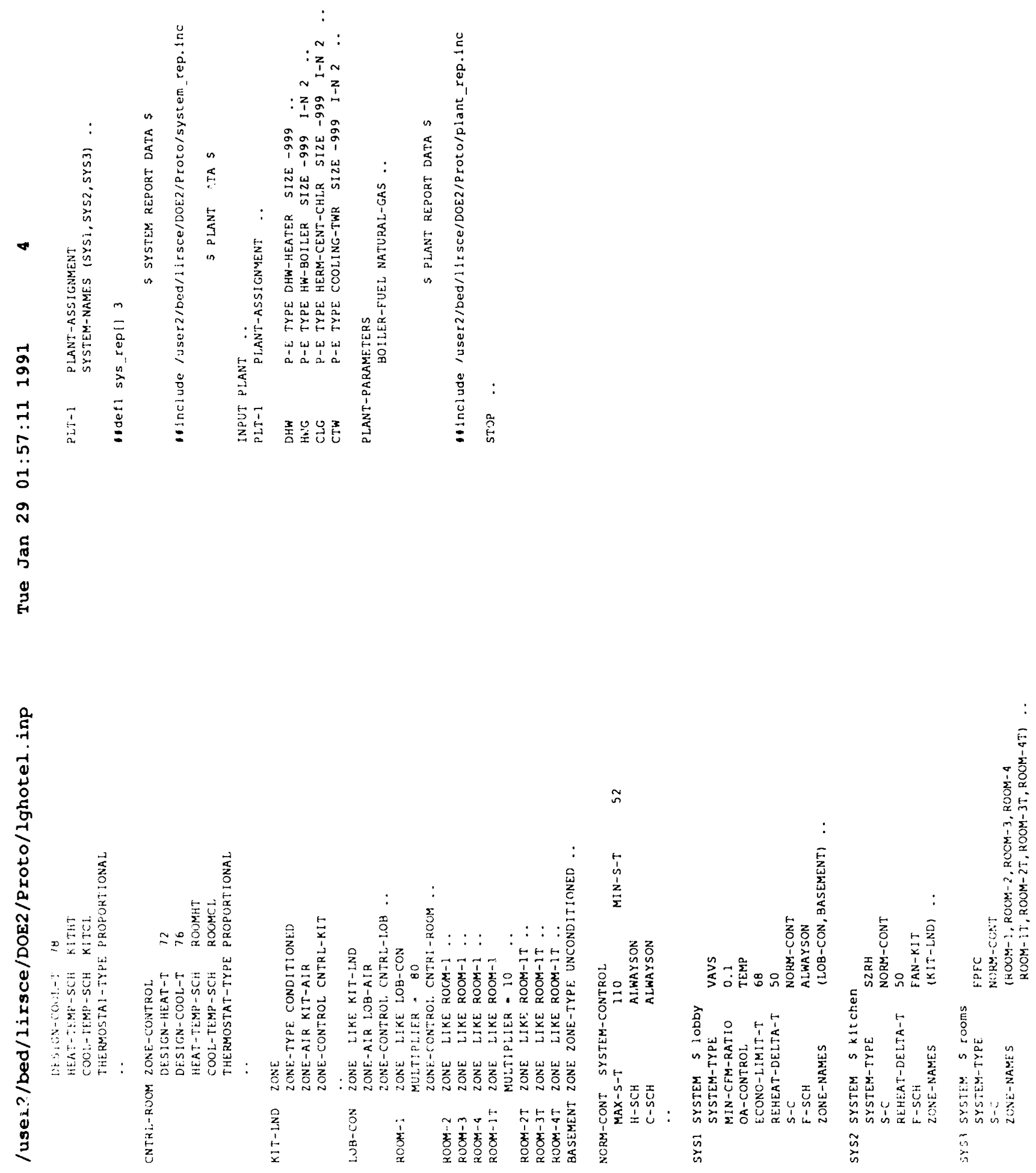


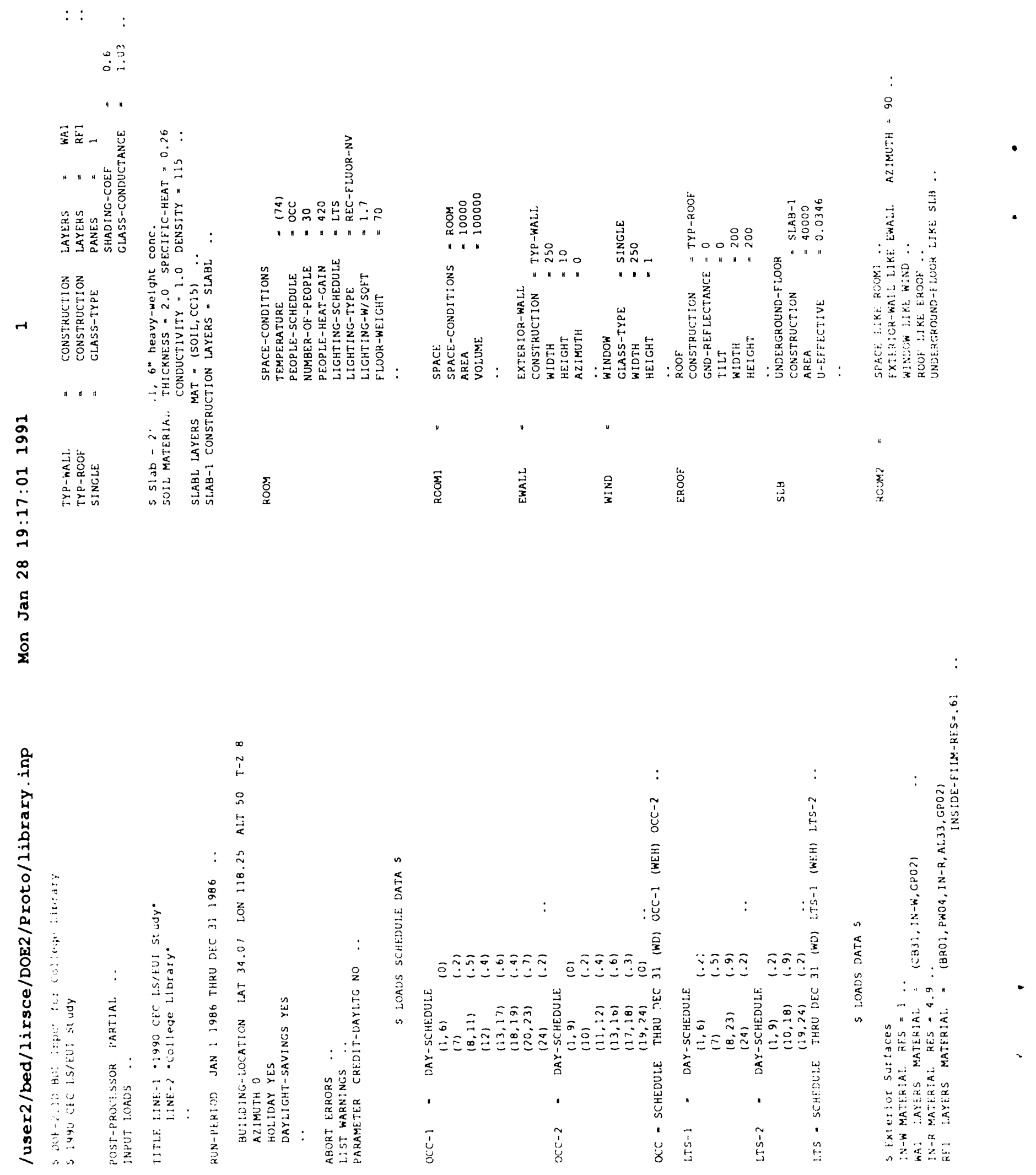



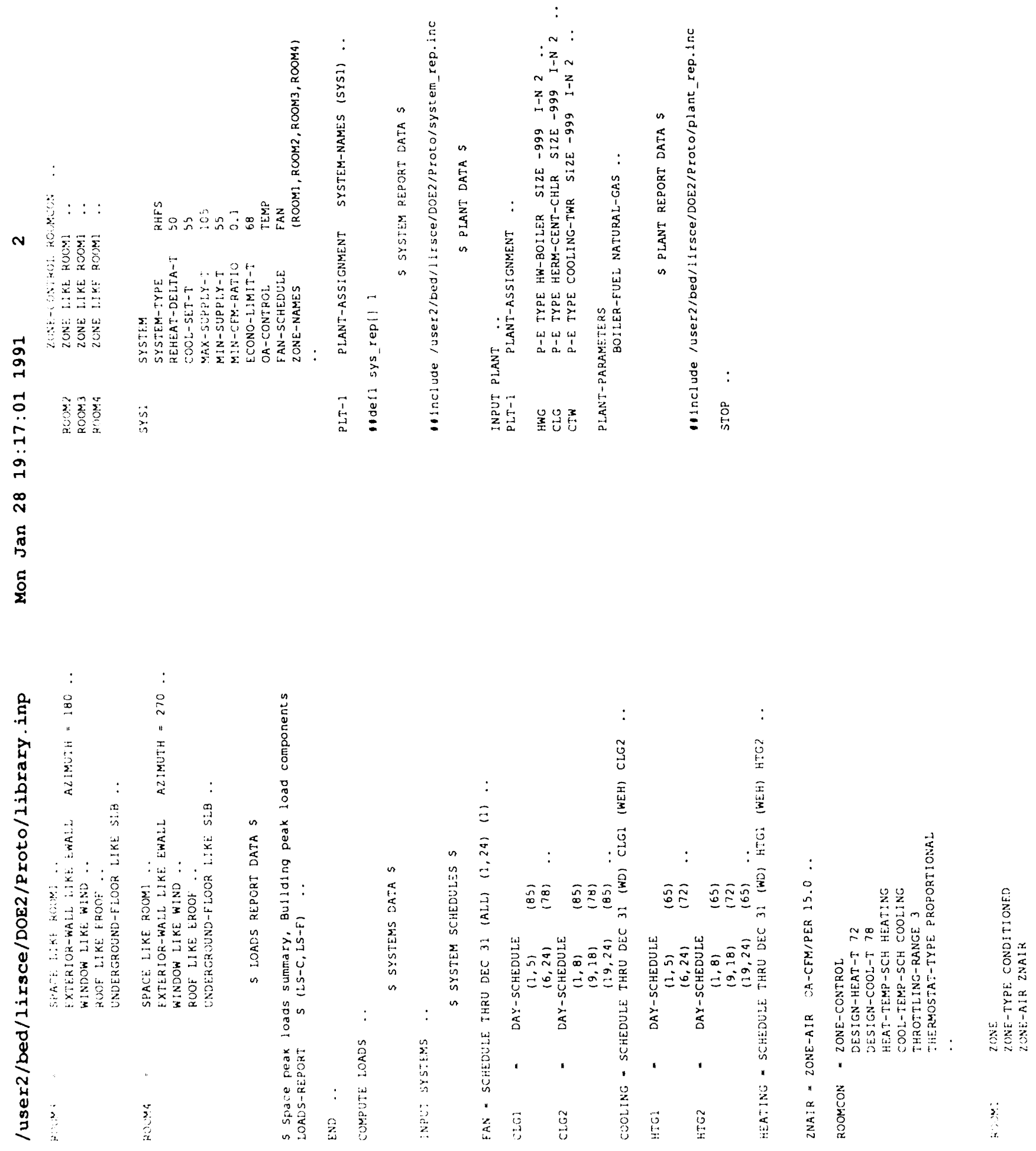

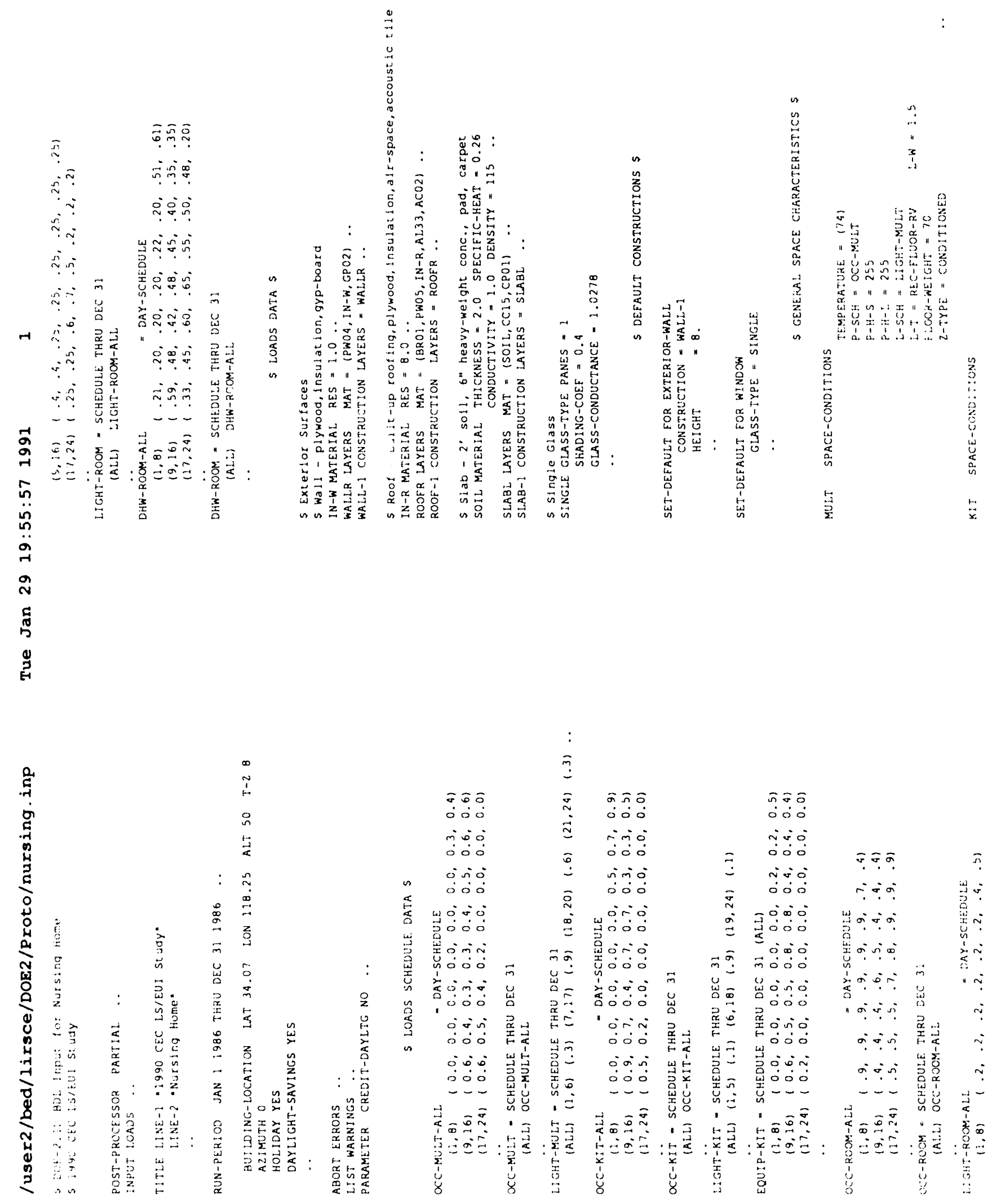

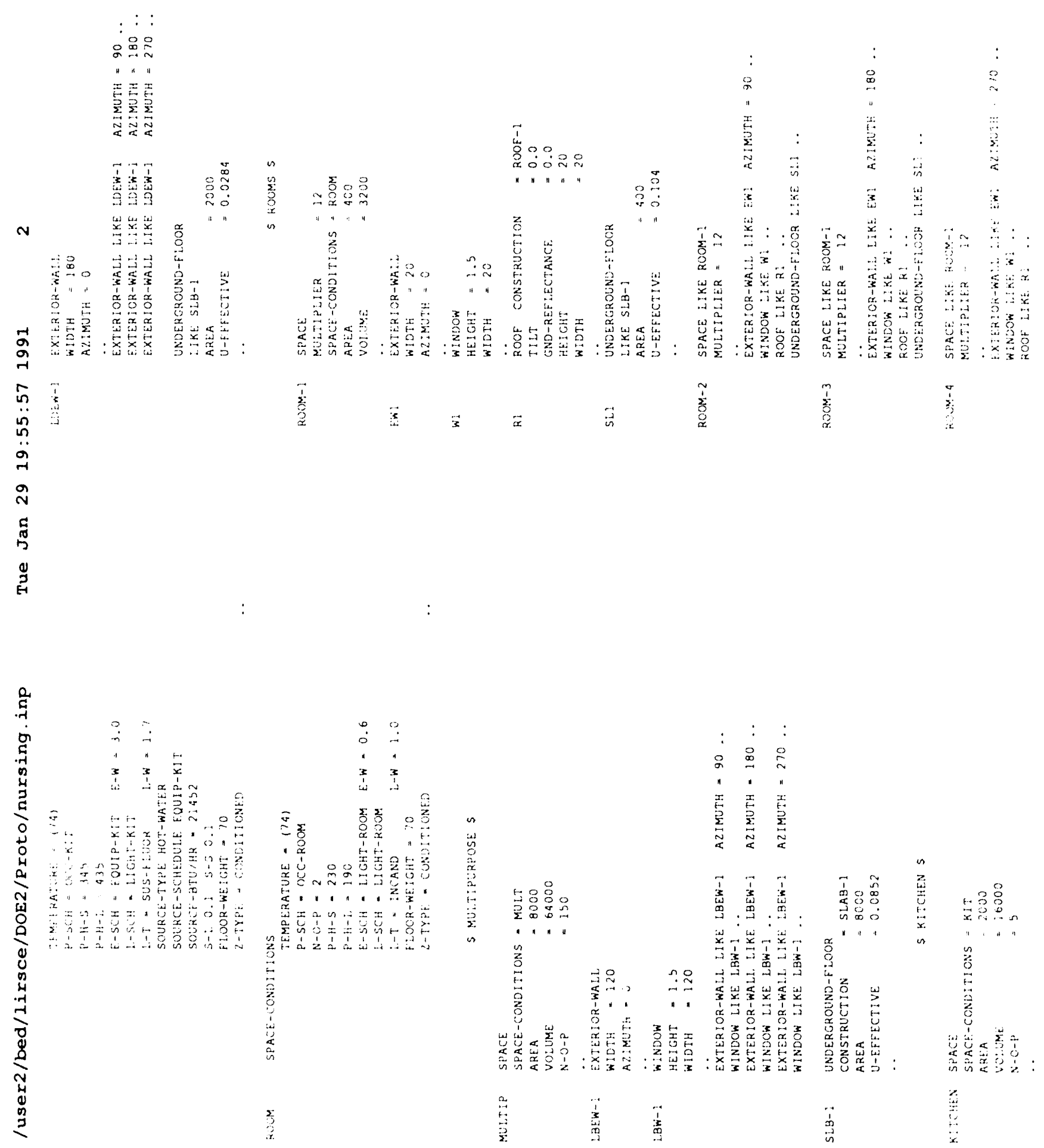

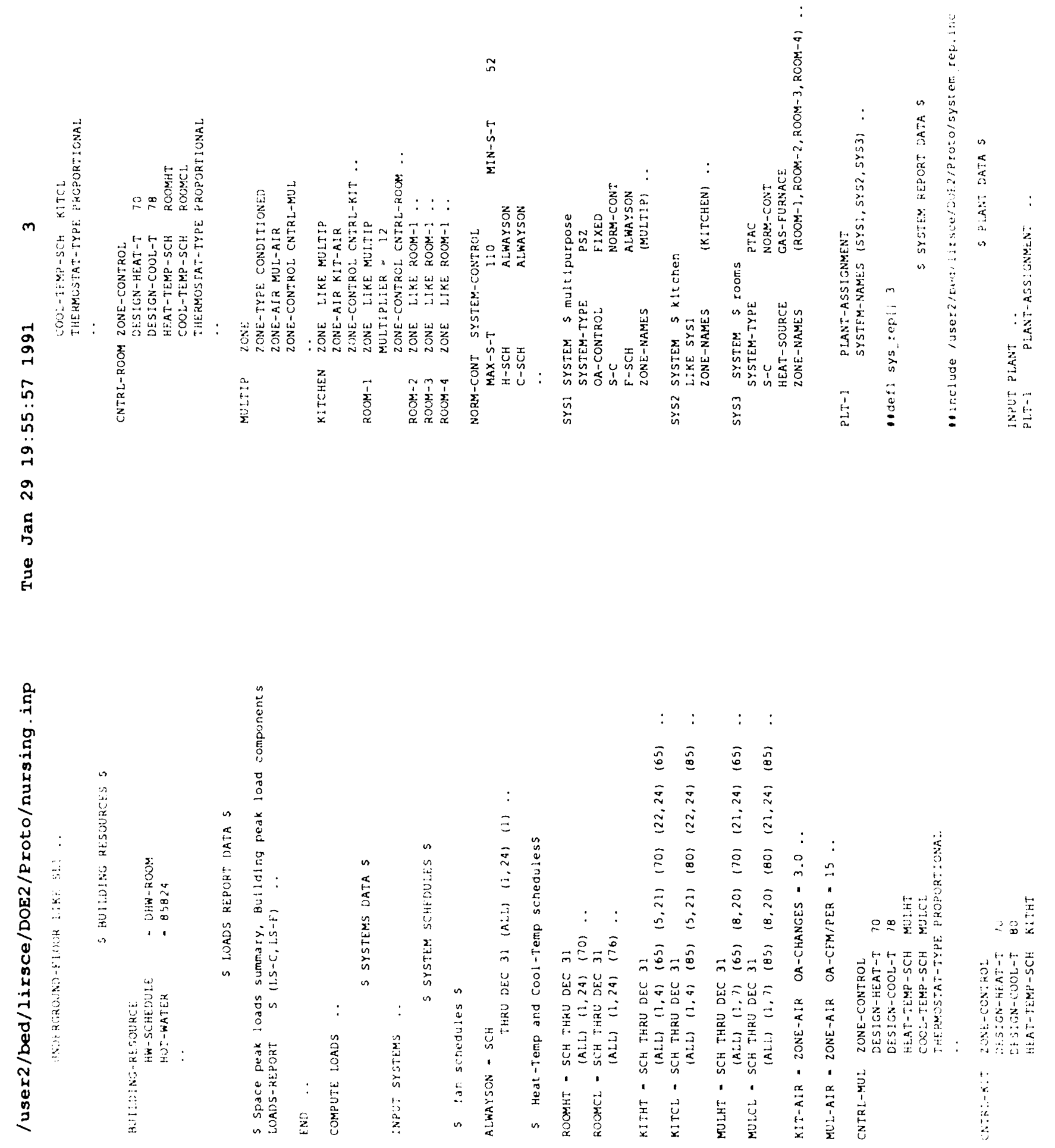


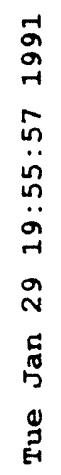

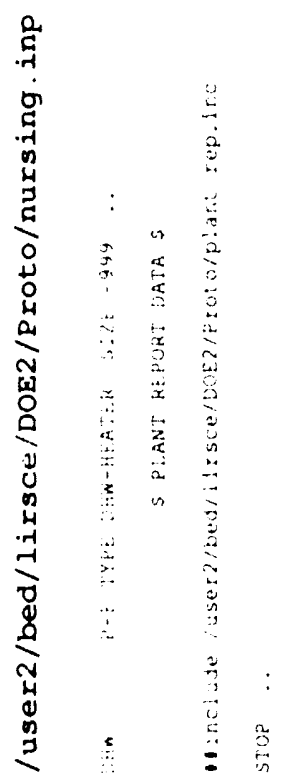



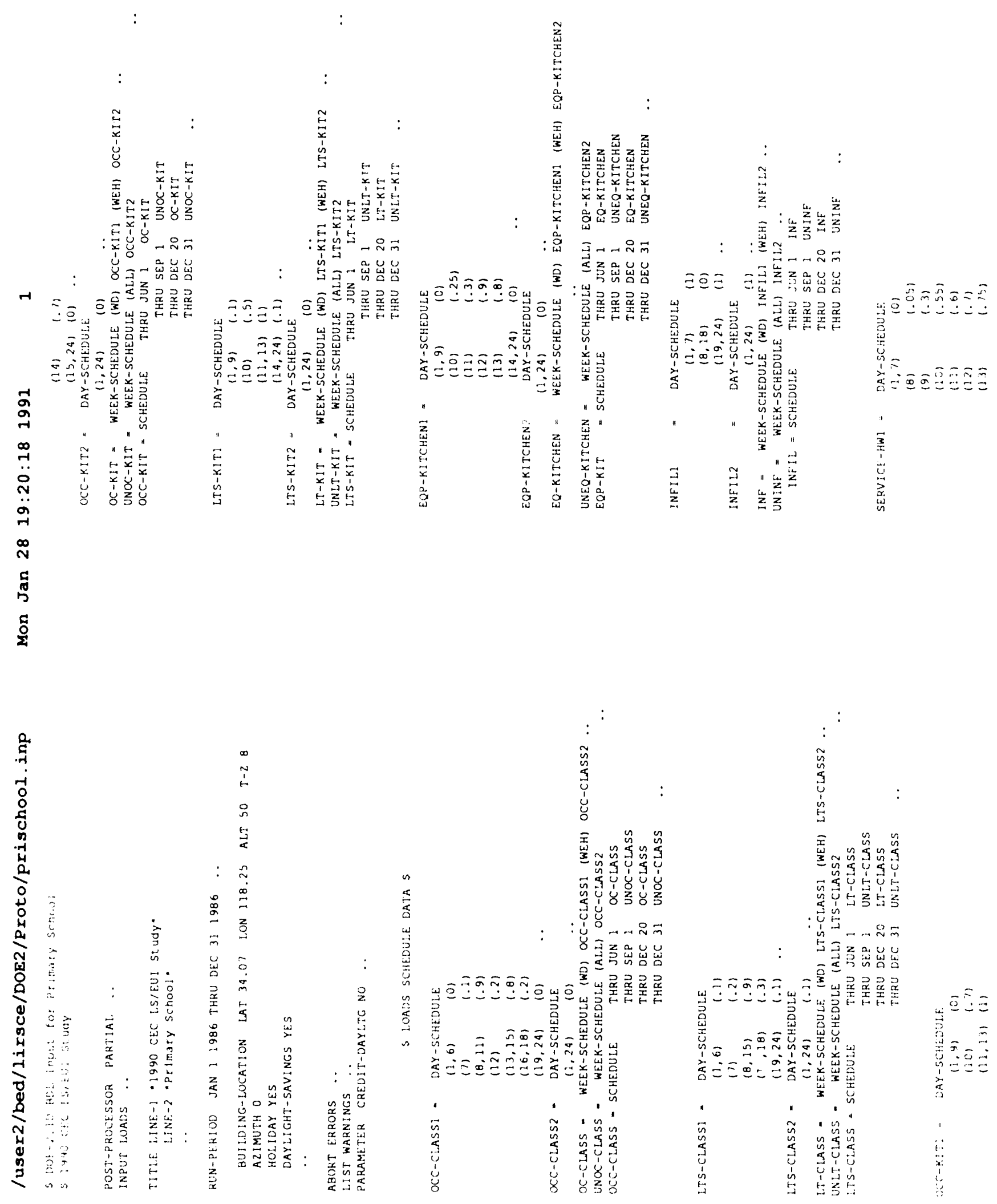


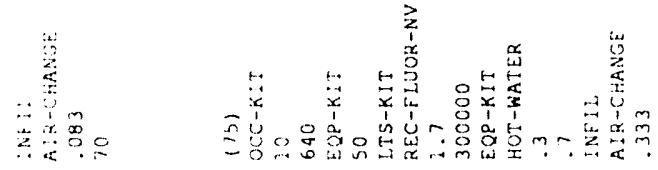

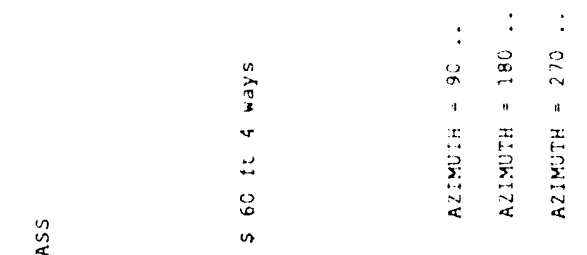

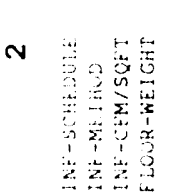

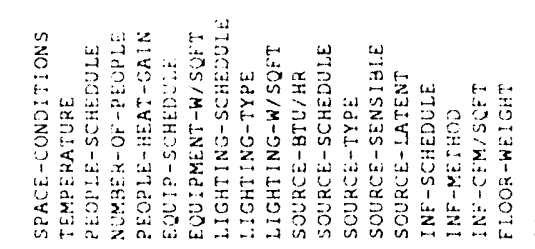

ă

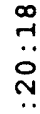

$$
\begin{aligned}
& a \\
& x \\
& i \\
& a \\
& \text { in }
\end{aligned}
$$

$\stackrel{n}{4}$

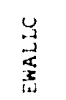

$\underset{2}{\stackrel{y}{2}}$

苟总势

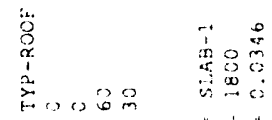

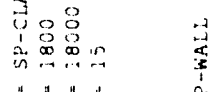

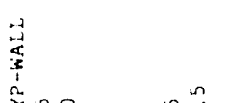

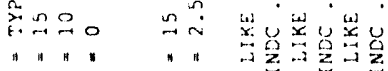

" " " " " "

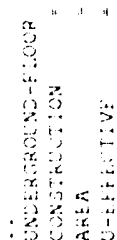

$\stackrel{\infty}{\sim}$

耍

章
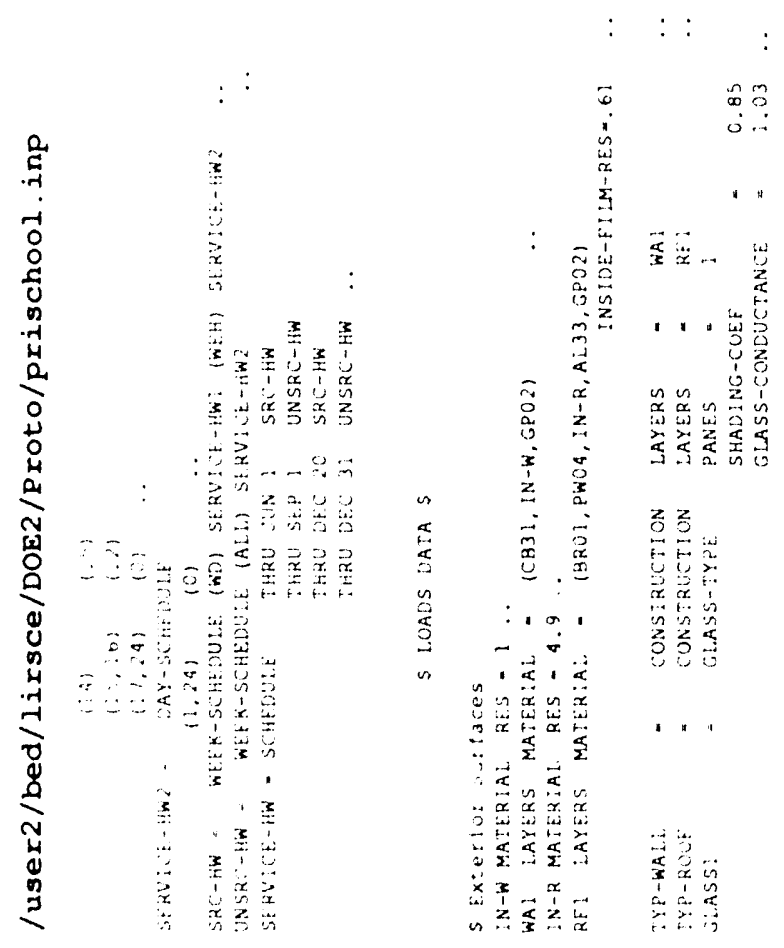

:
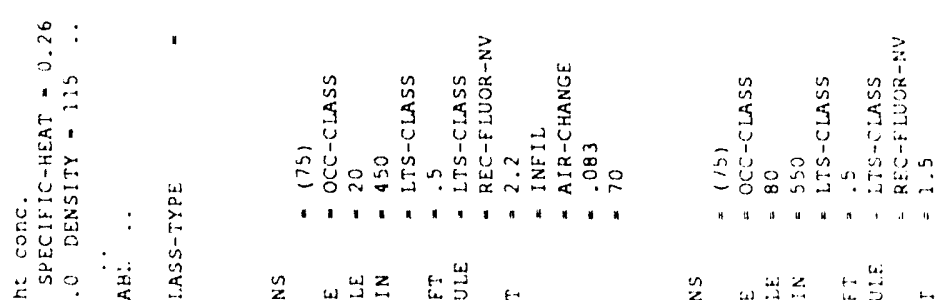

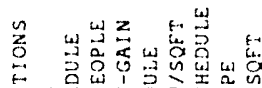

要过沓 E.

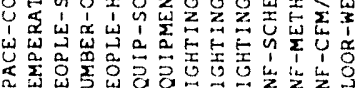

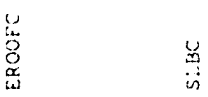

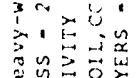

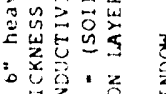

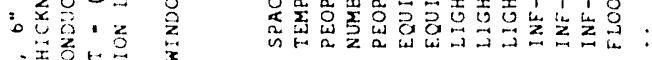

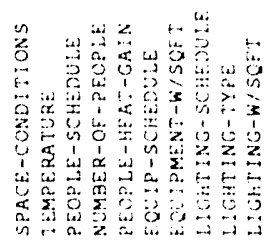

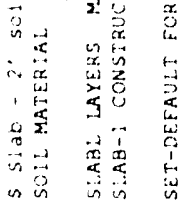

$n$
0
3
$y$
$\vdots$
$\vdots$
$i$

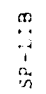



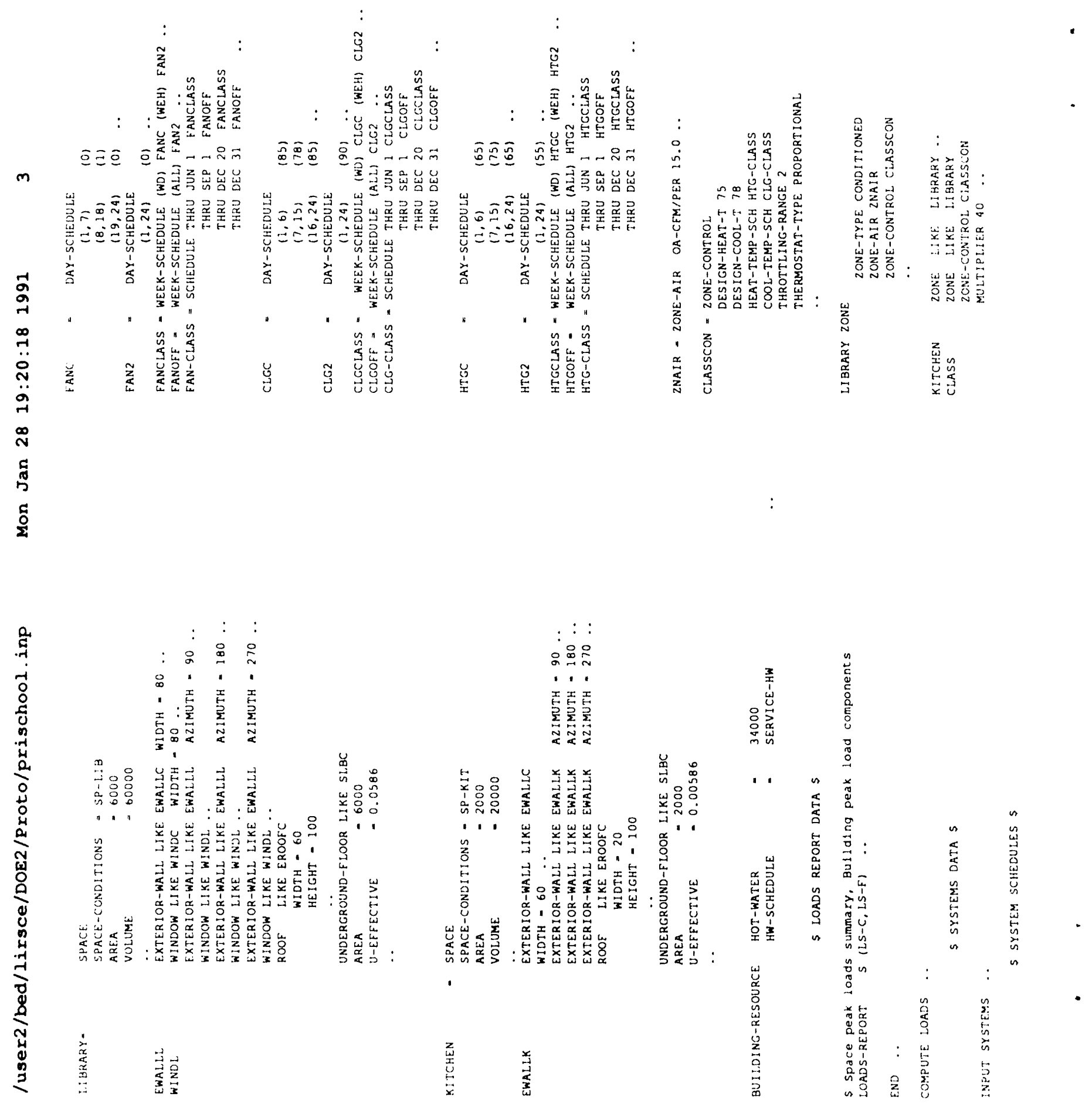


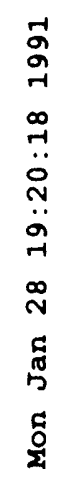

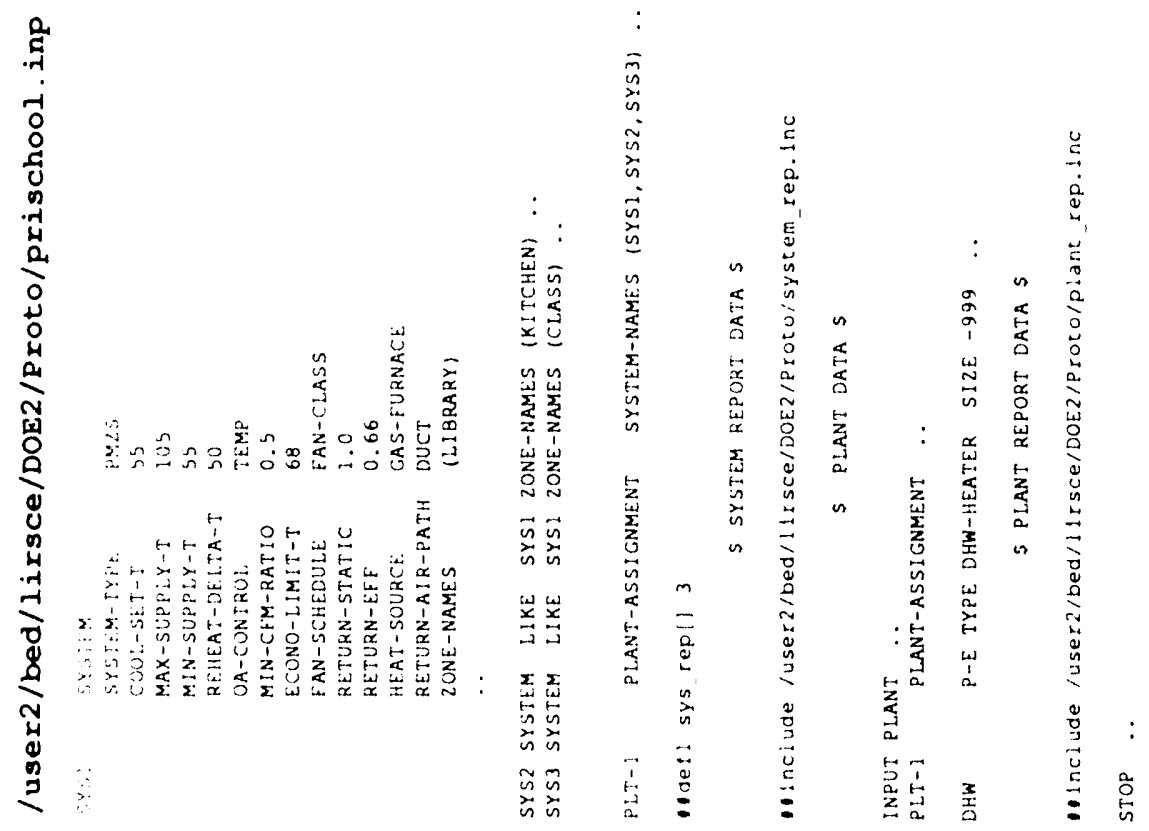



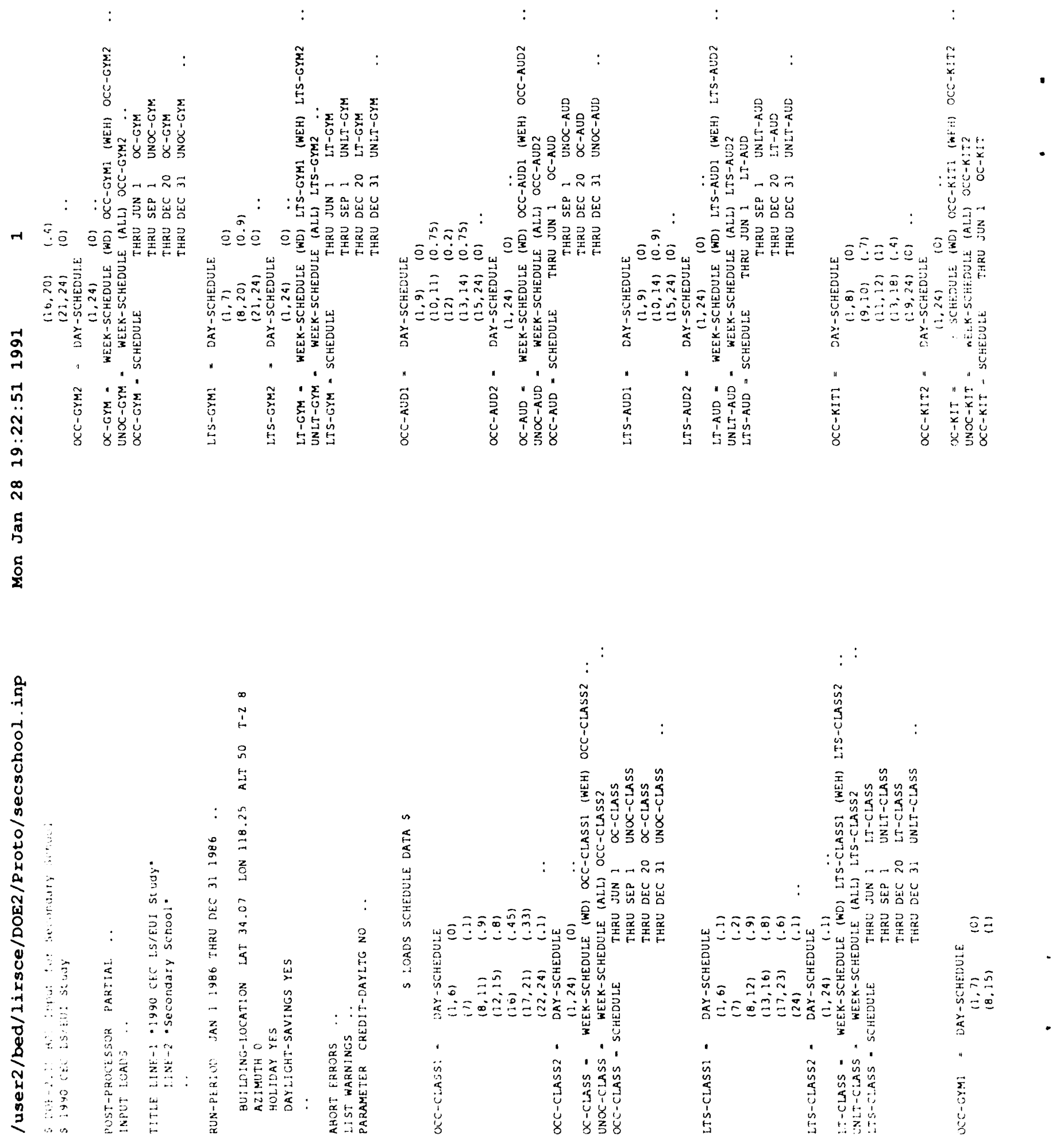

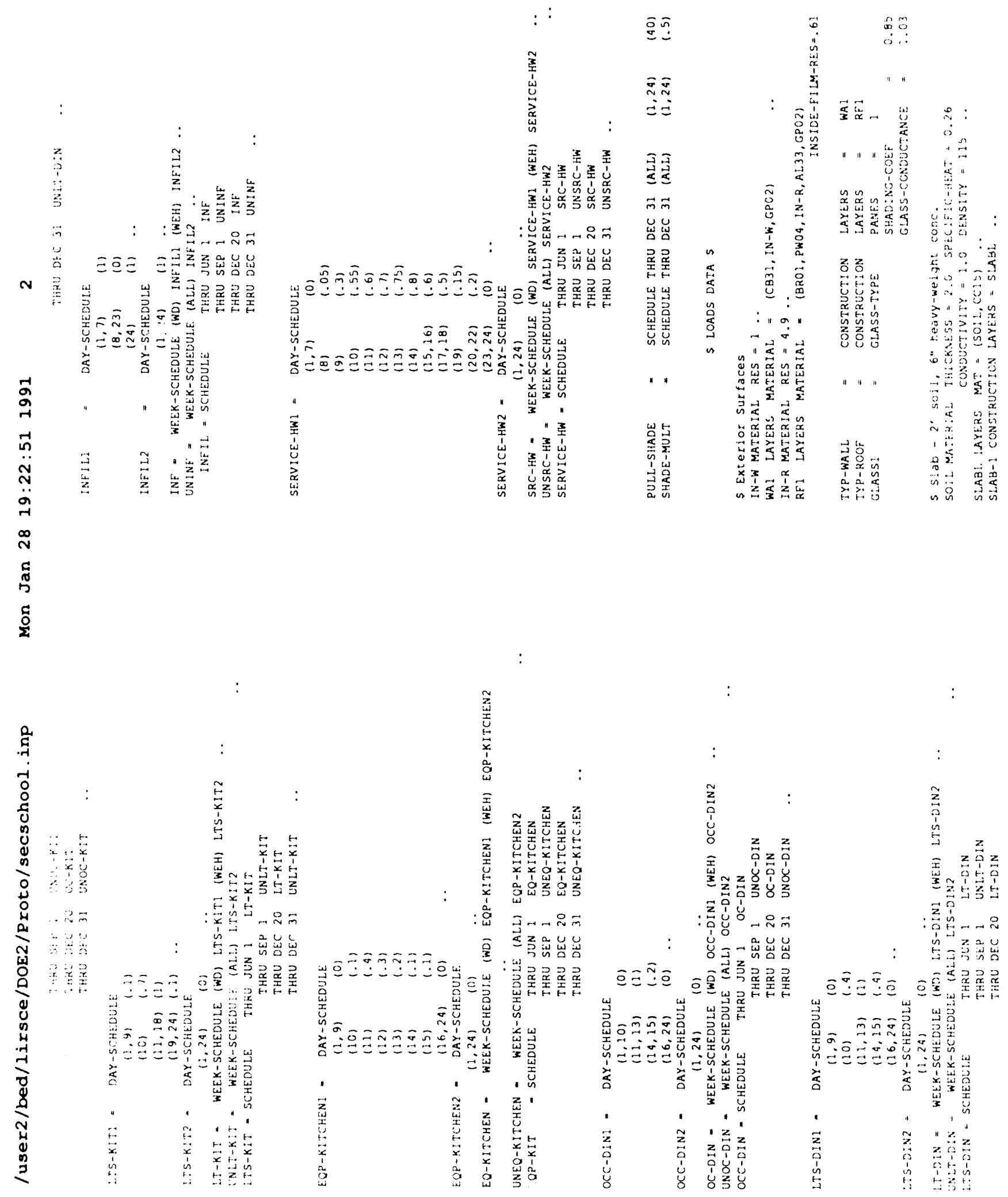

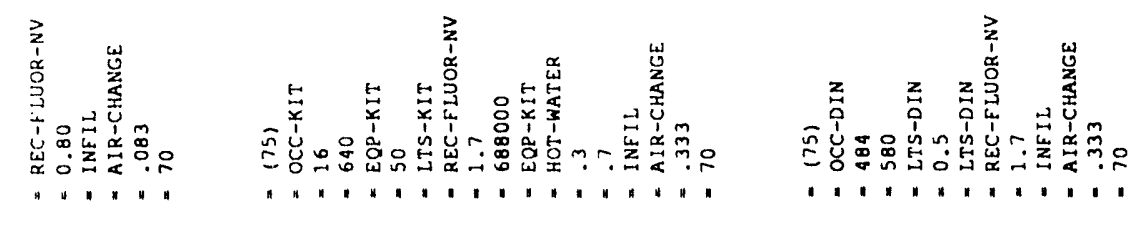

1
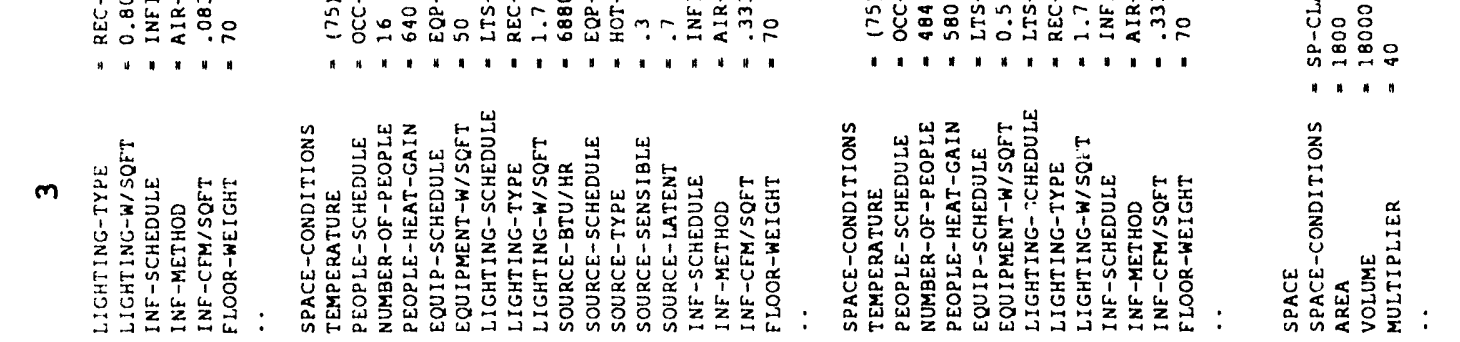

Inse

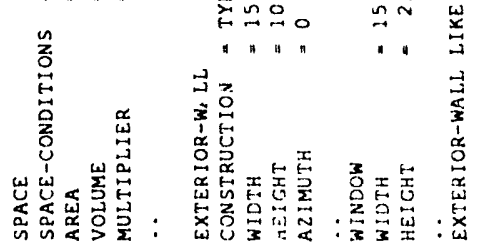

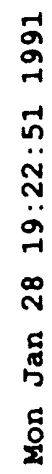

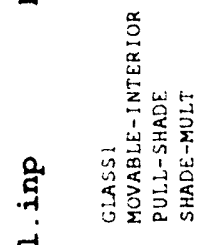

5
$\vec{x}$
0
0
in

$z$
$i$
$\vdots$
ì

离

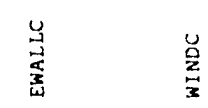

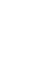

.

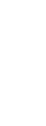

军

ㅇ․…

高

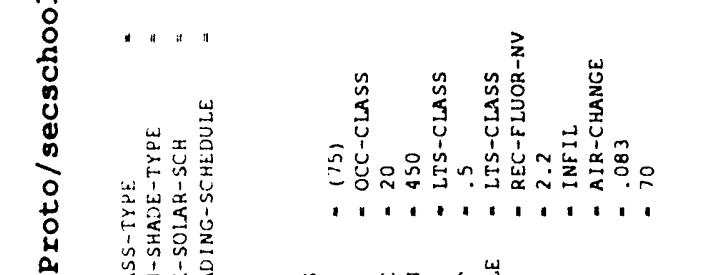

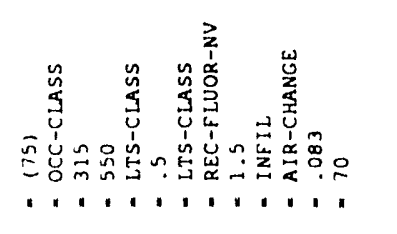

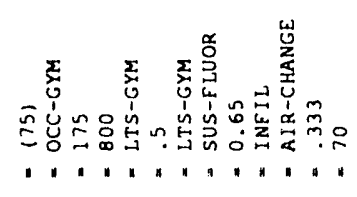

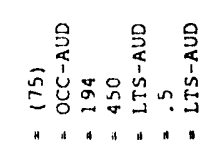
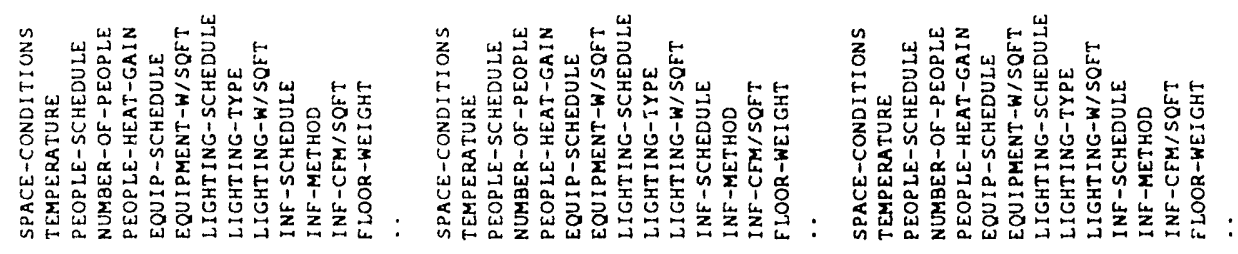

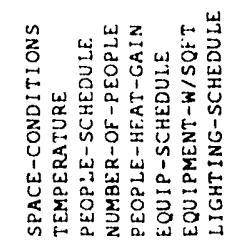

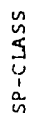

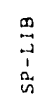

$x$
$i$
$i$
$i$

3
$\substack{1 \\ 1 \\ i}$
$i$ 

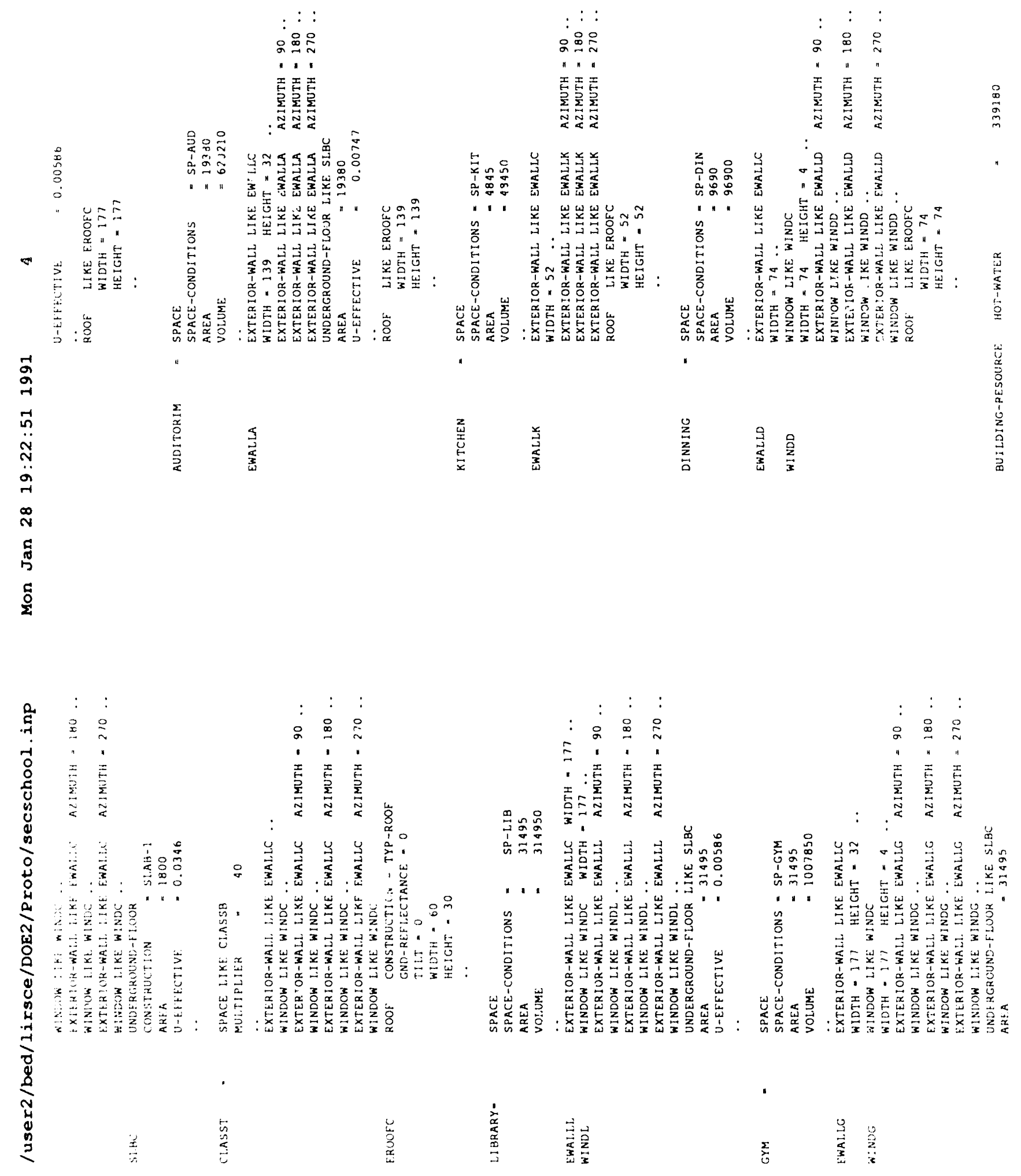

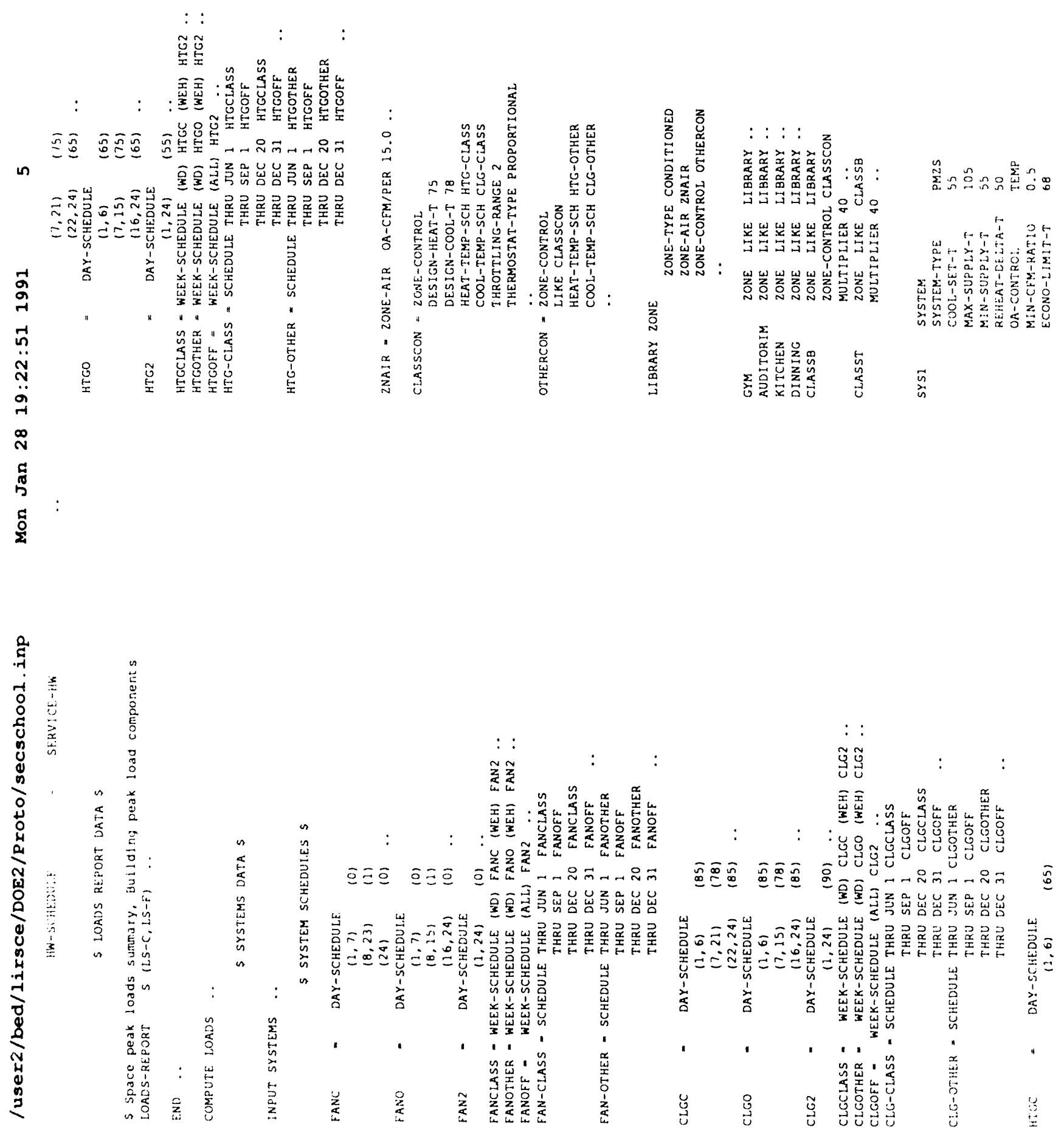


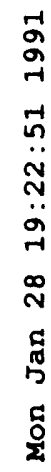

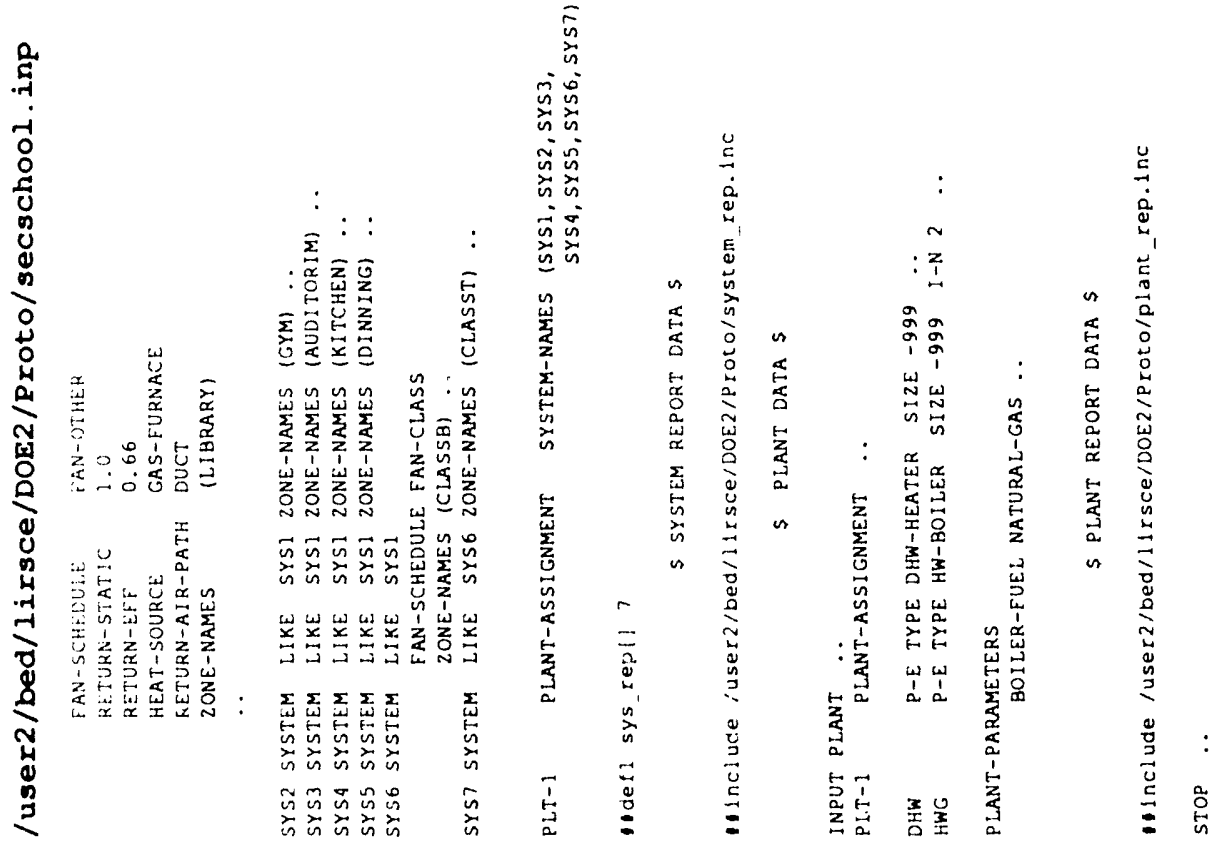



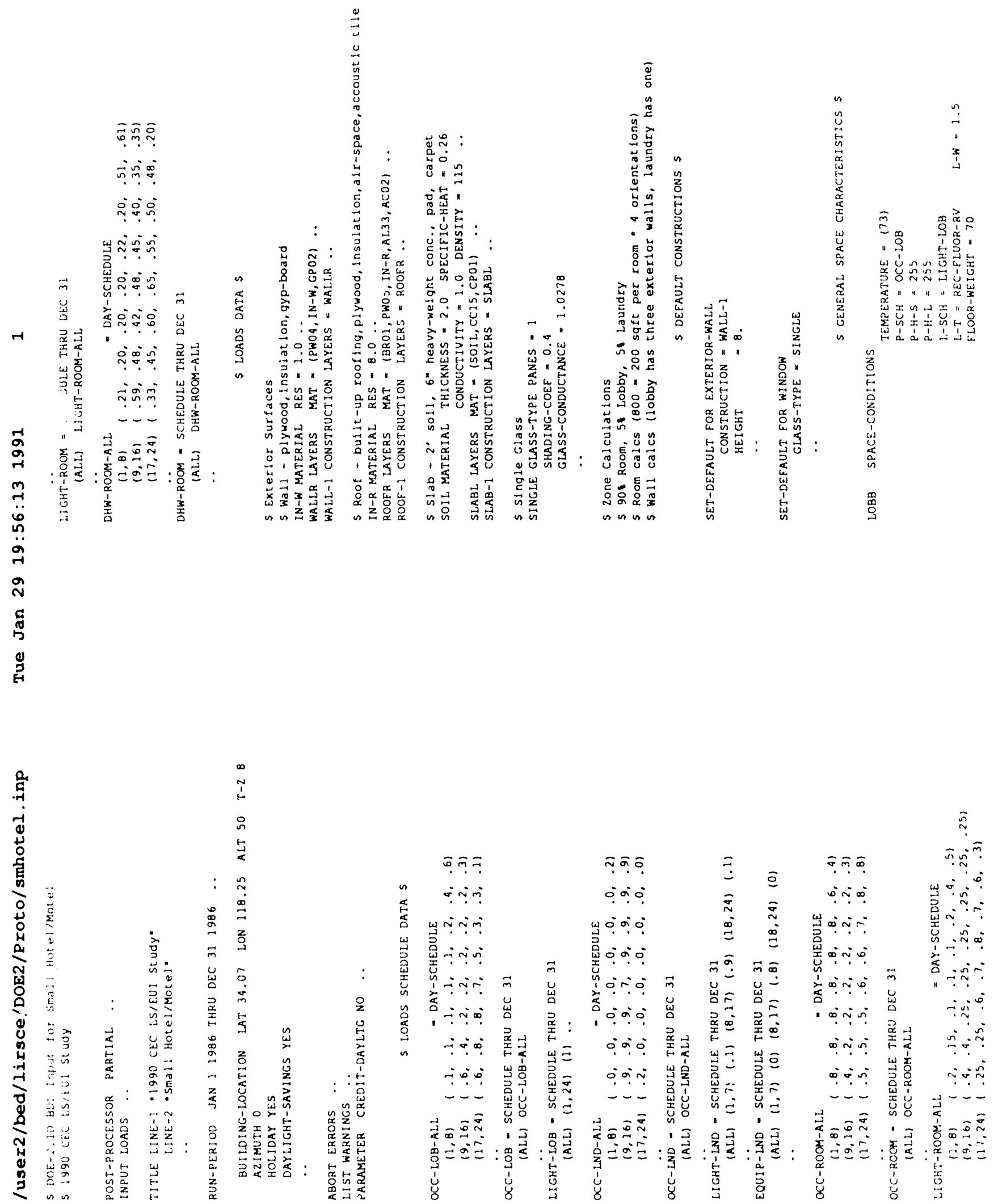

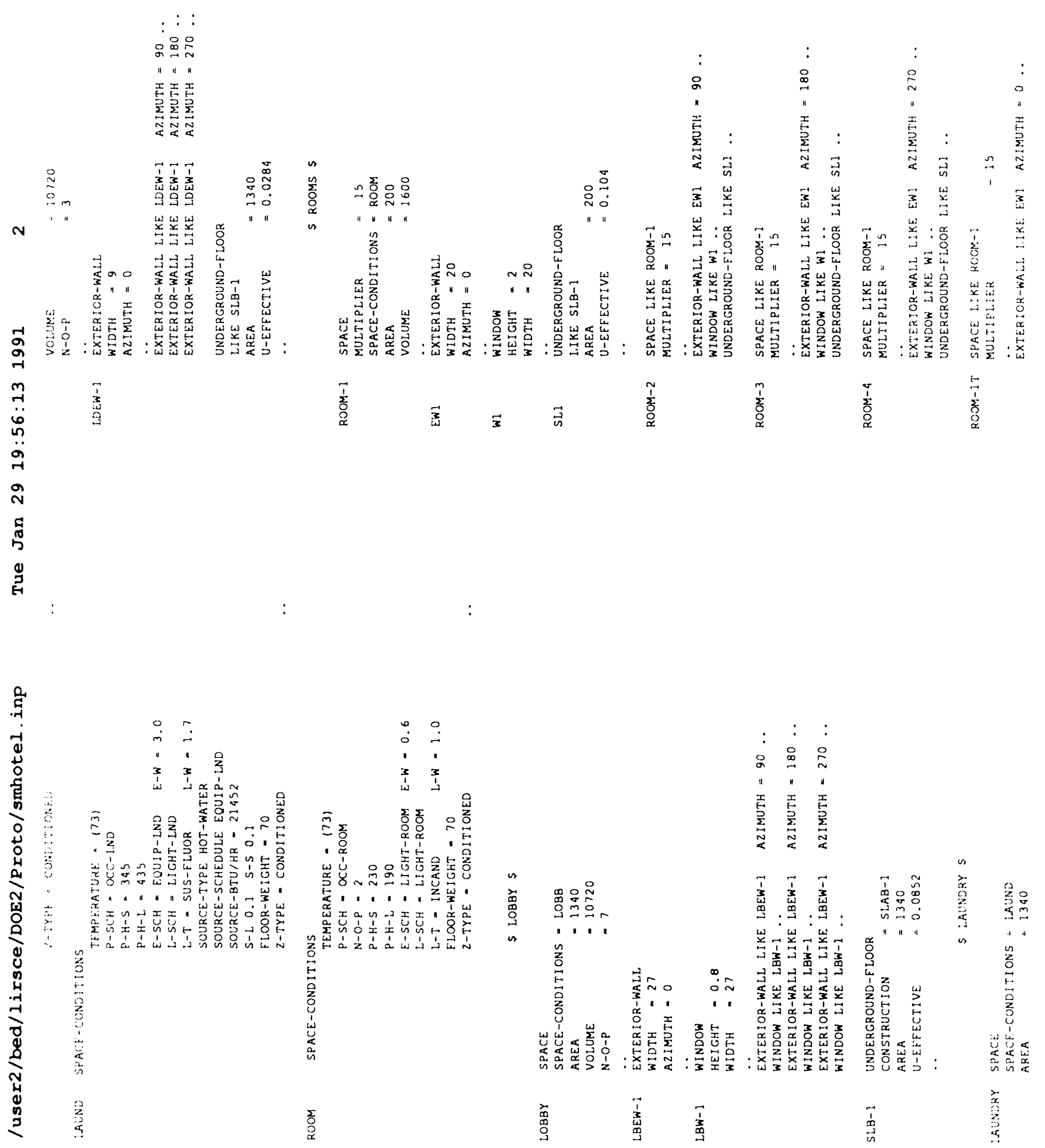


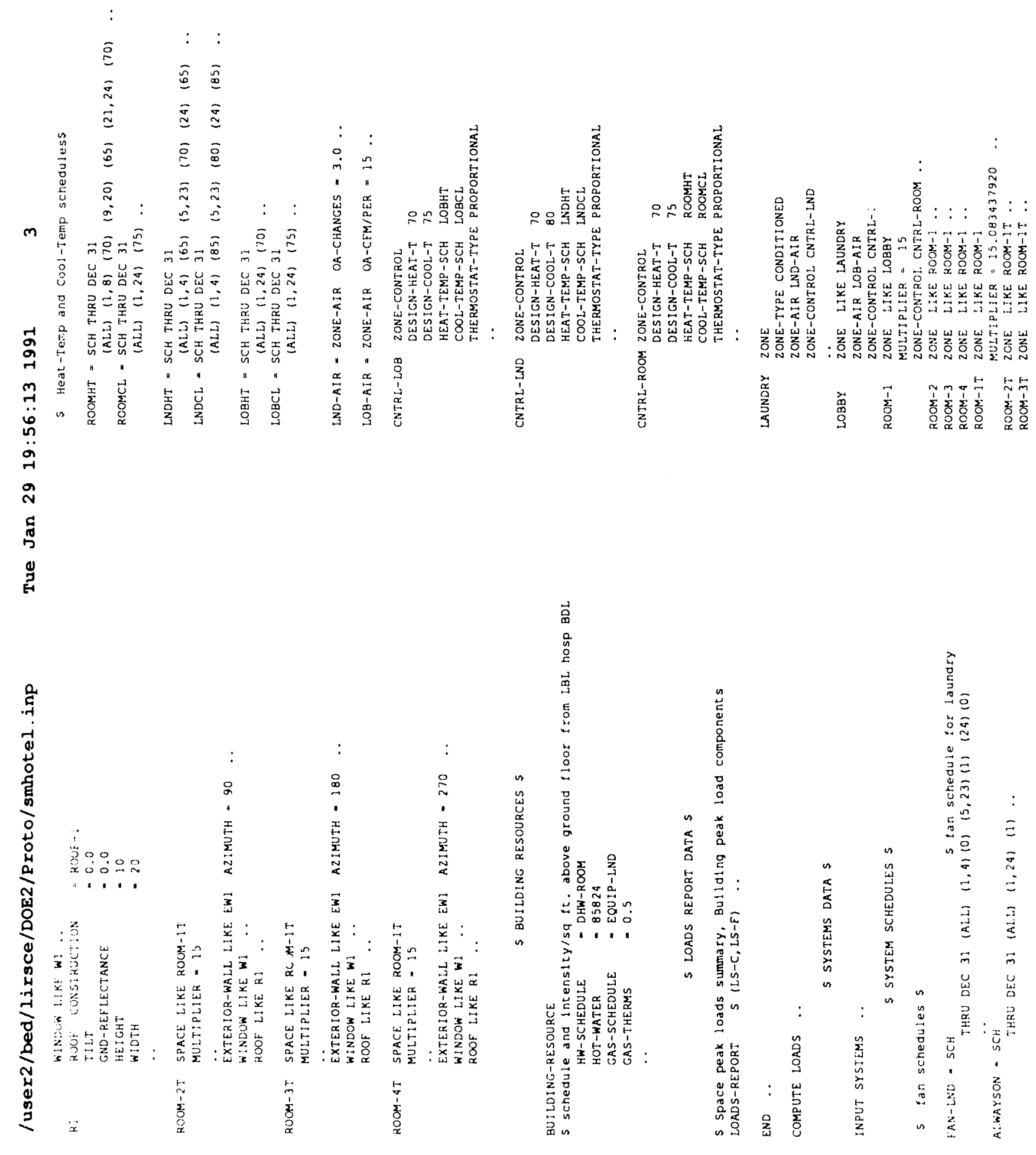




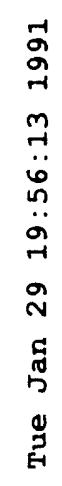

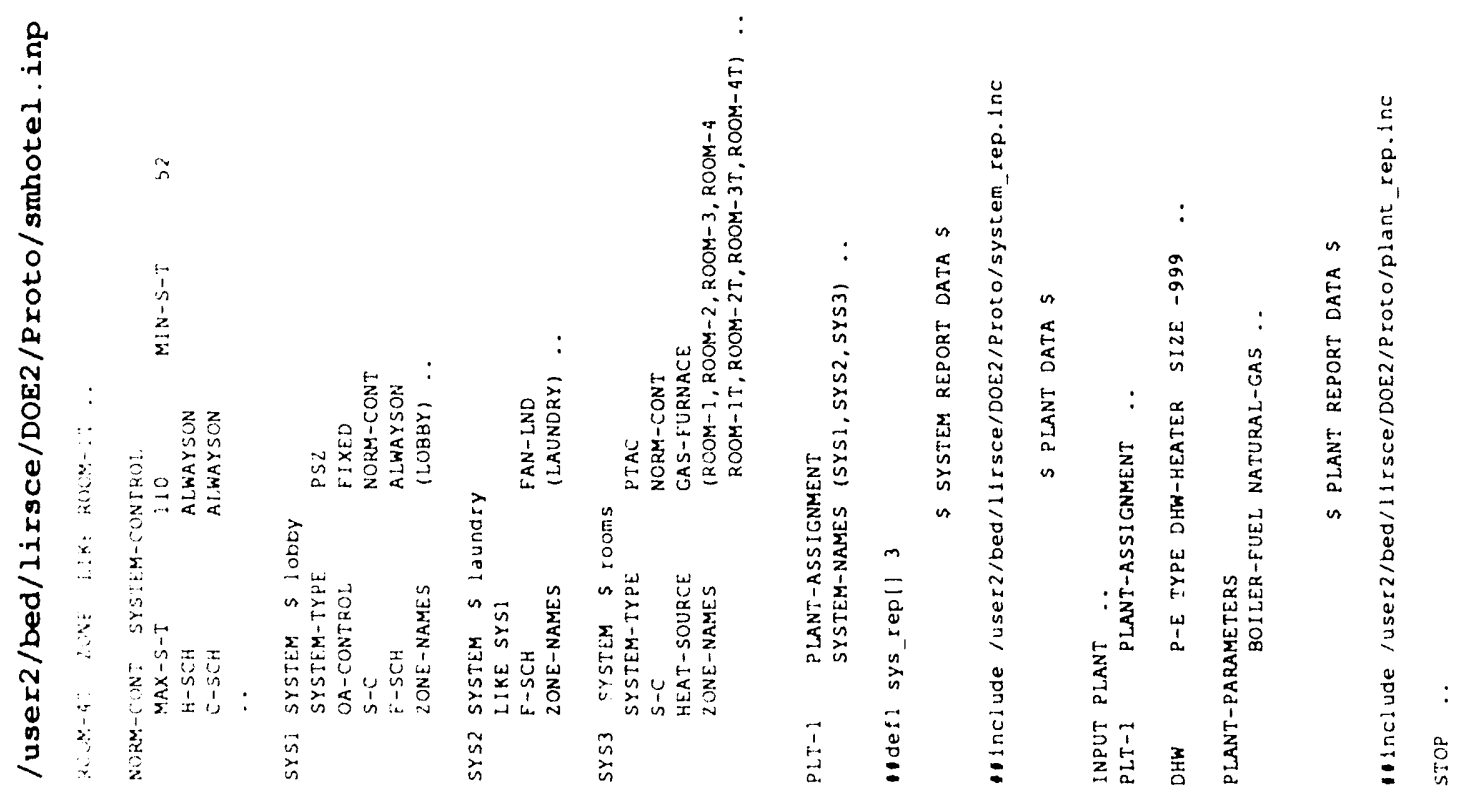


Appendix C. Summaries of LRD 
Average Weekday Profiles for Schools
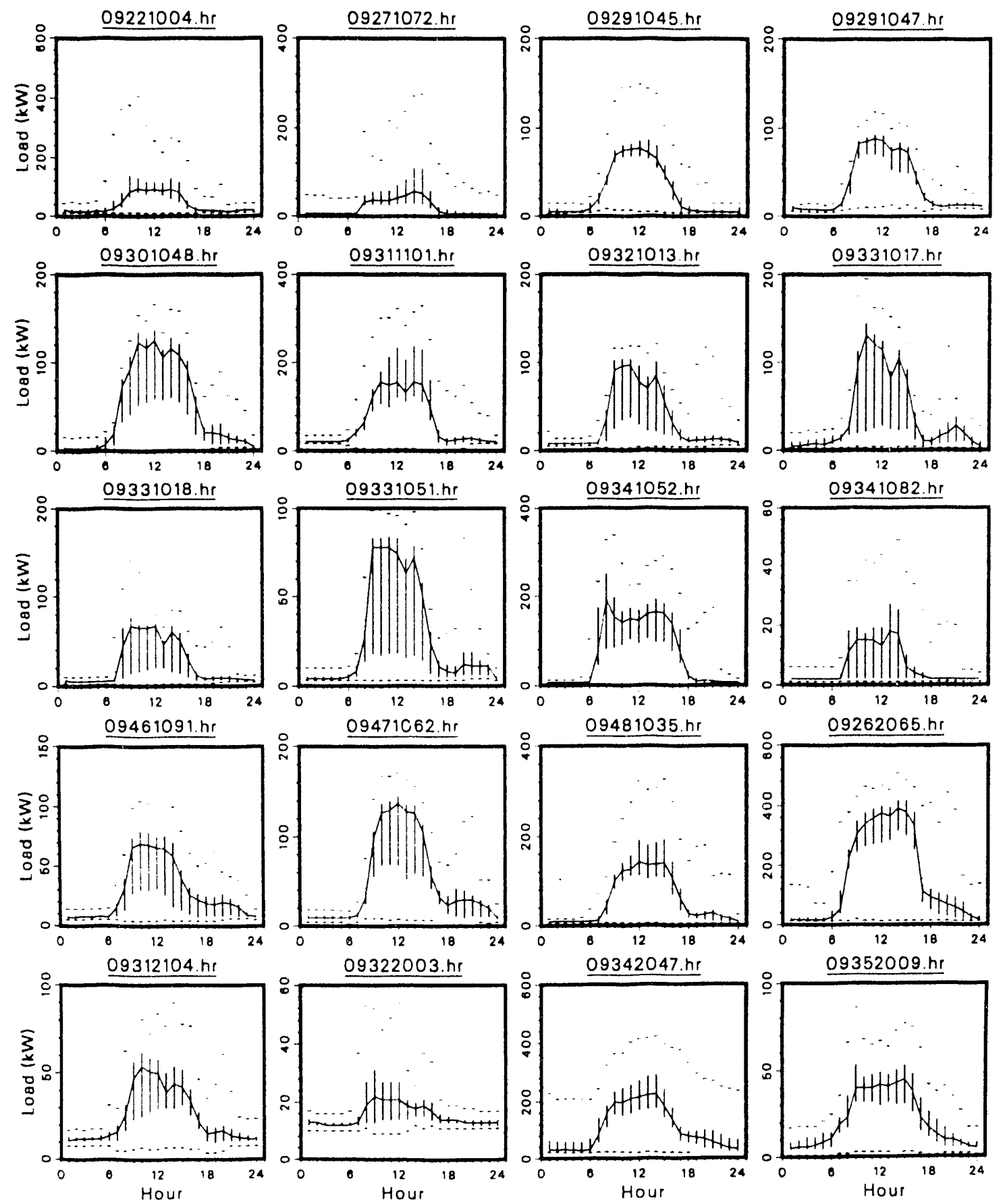


\section{Average Weekday Profiles for Schools}
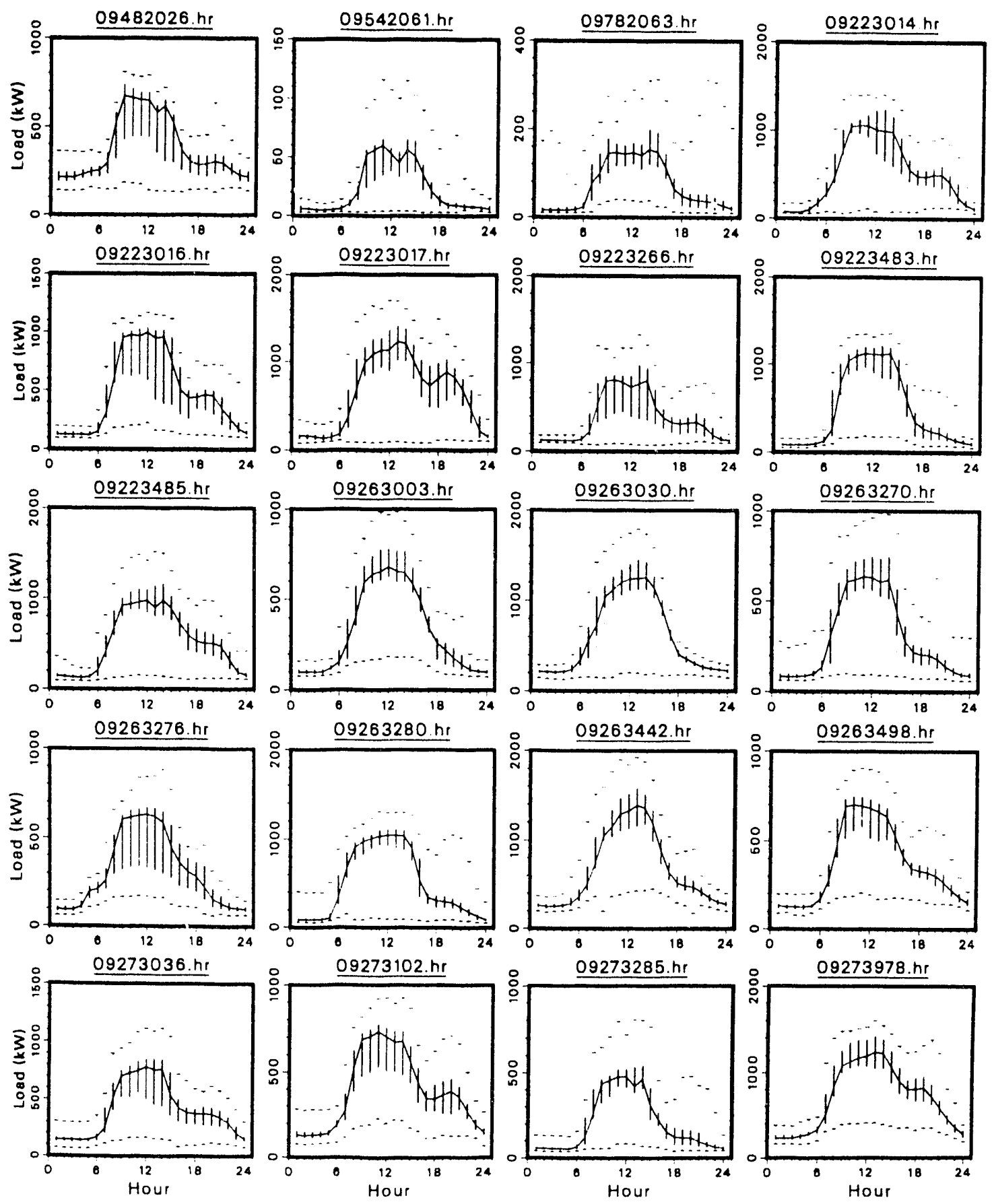


\section{Average Weekday Profiles for Schools}
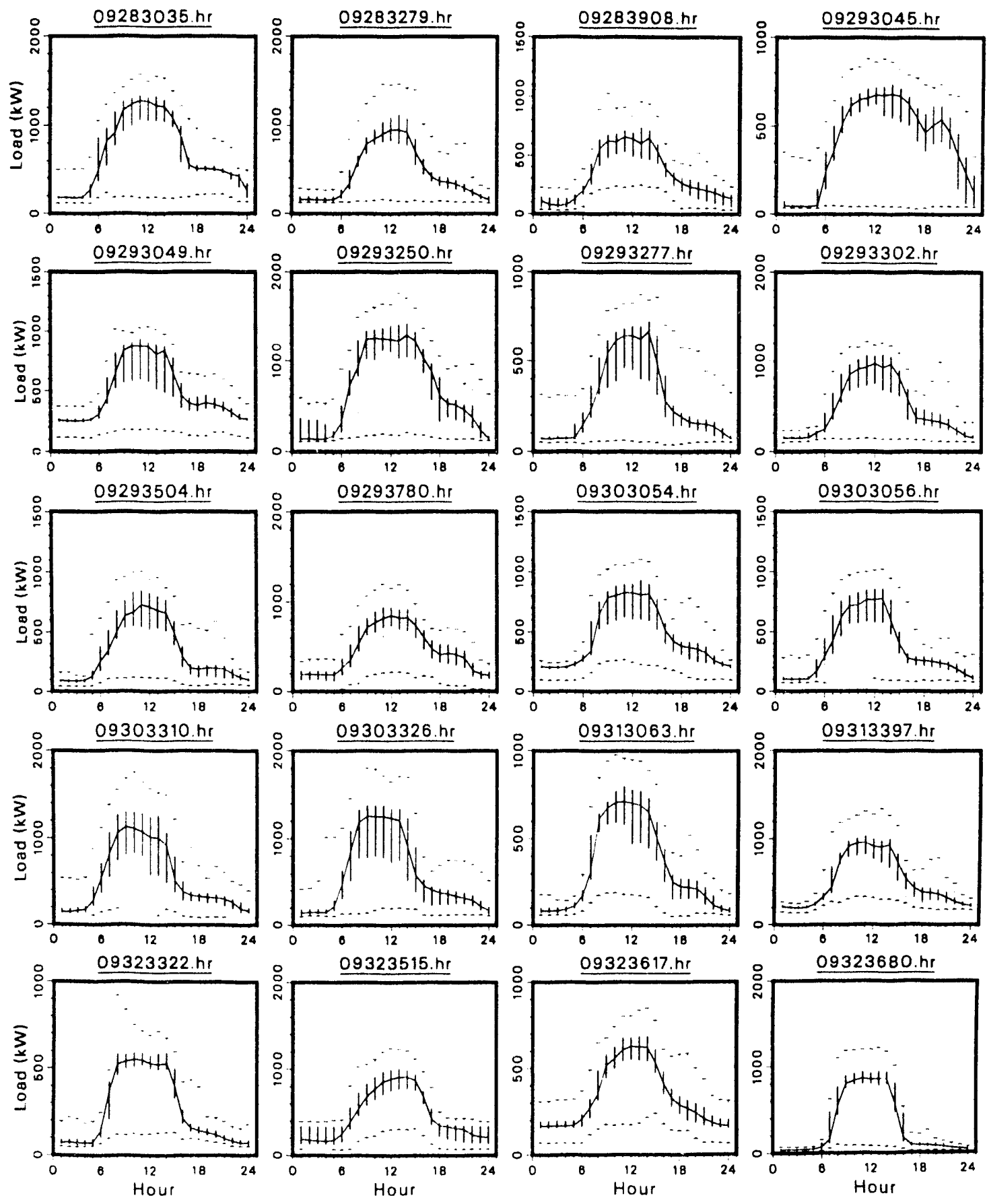
Average Weekday Profiles for Schools
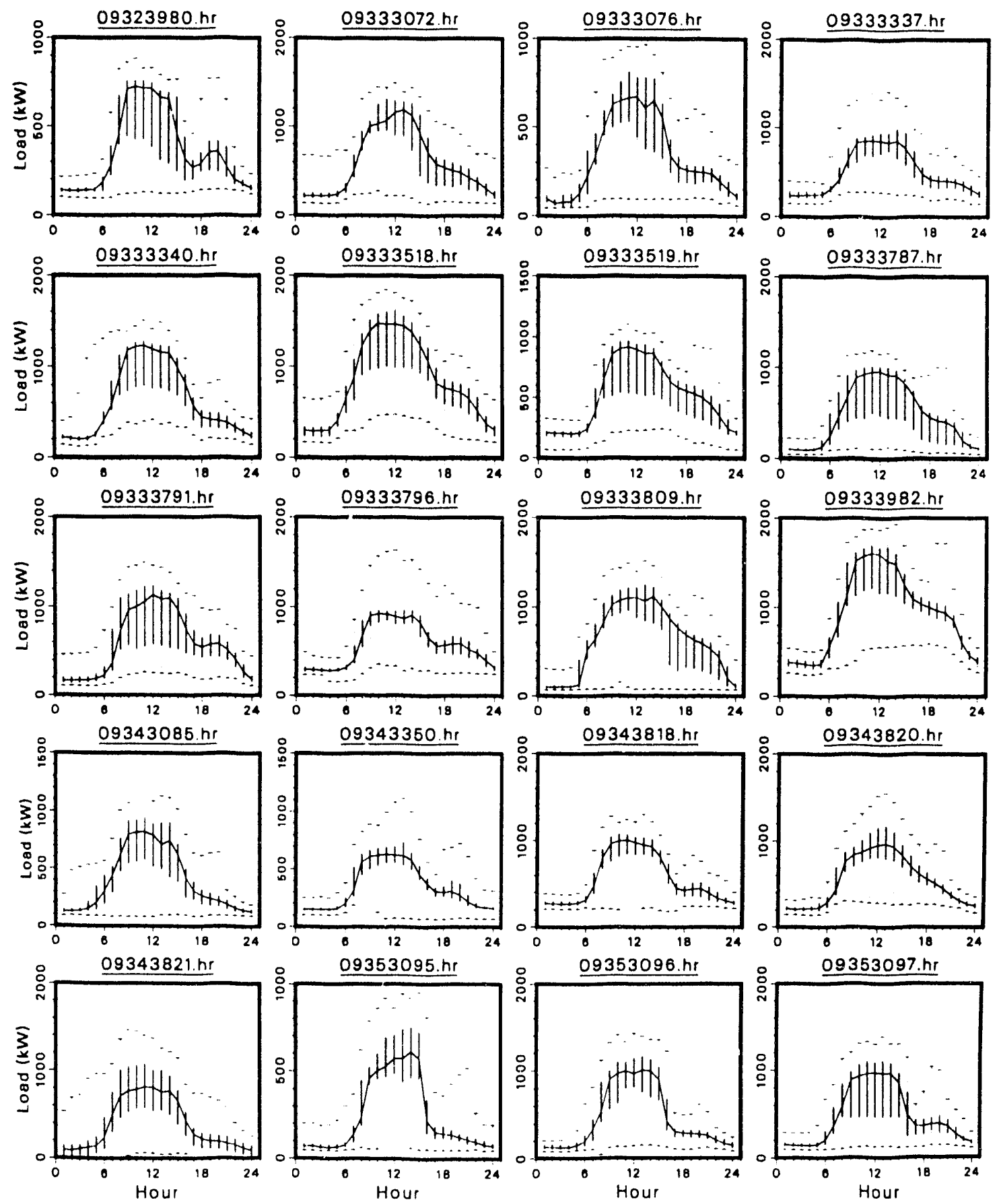


\section{Average Weekday Profiles for Schools}
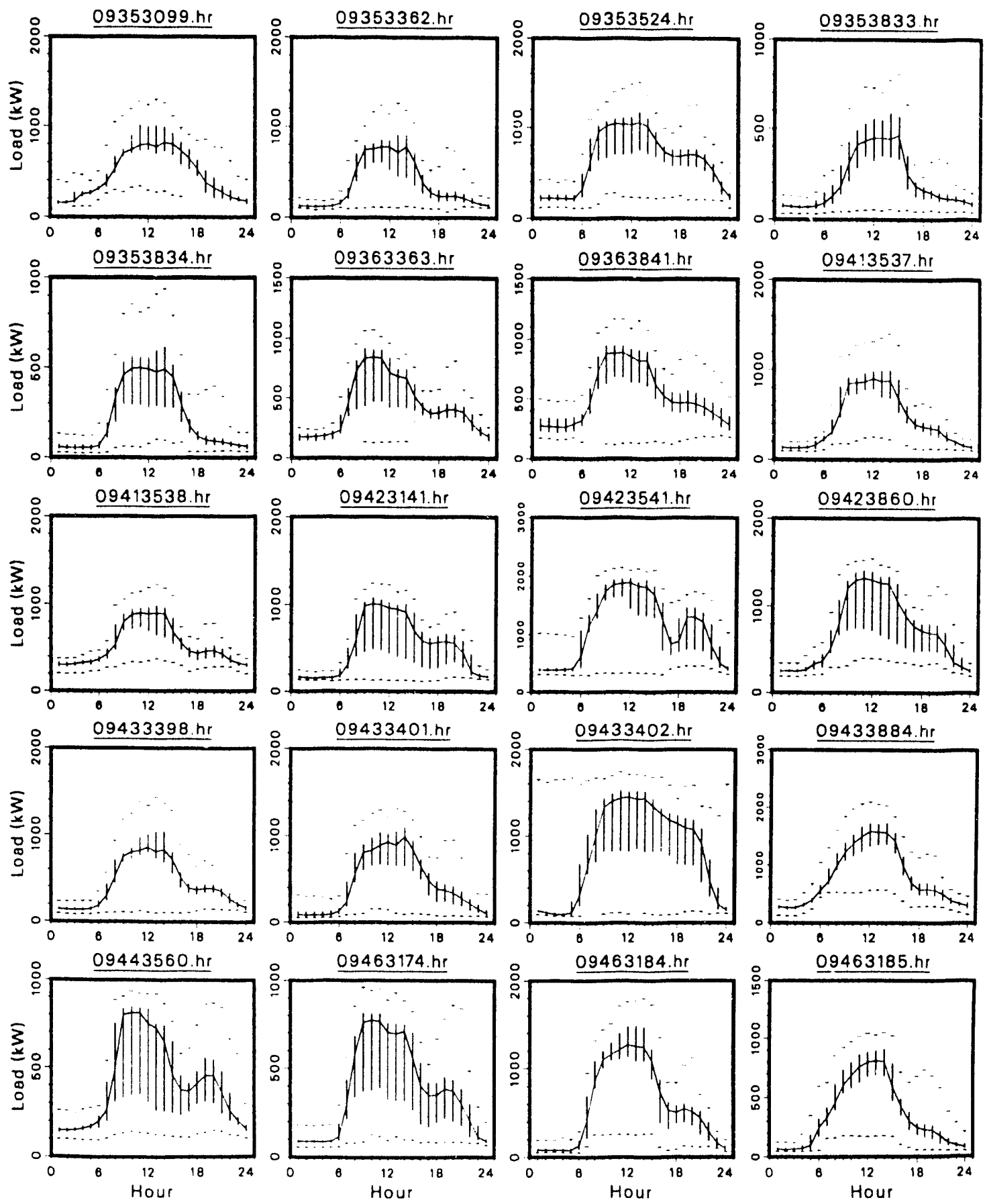


\section{Average Weekday Profiles for Schools}
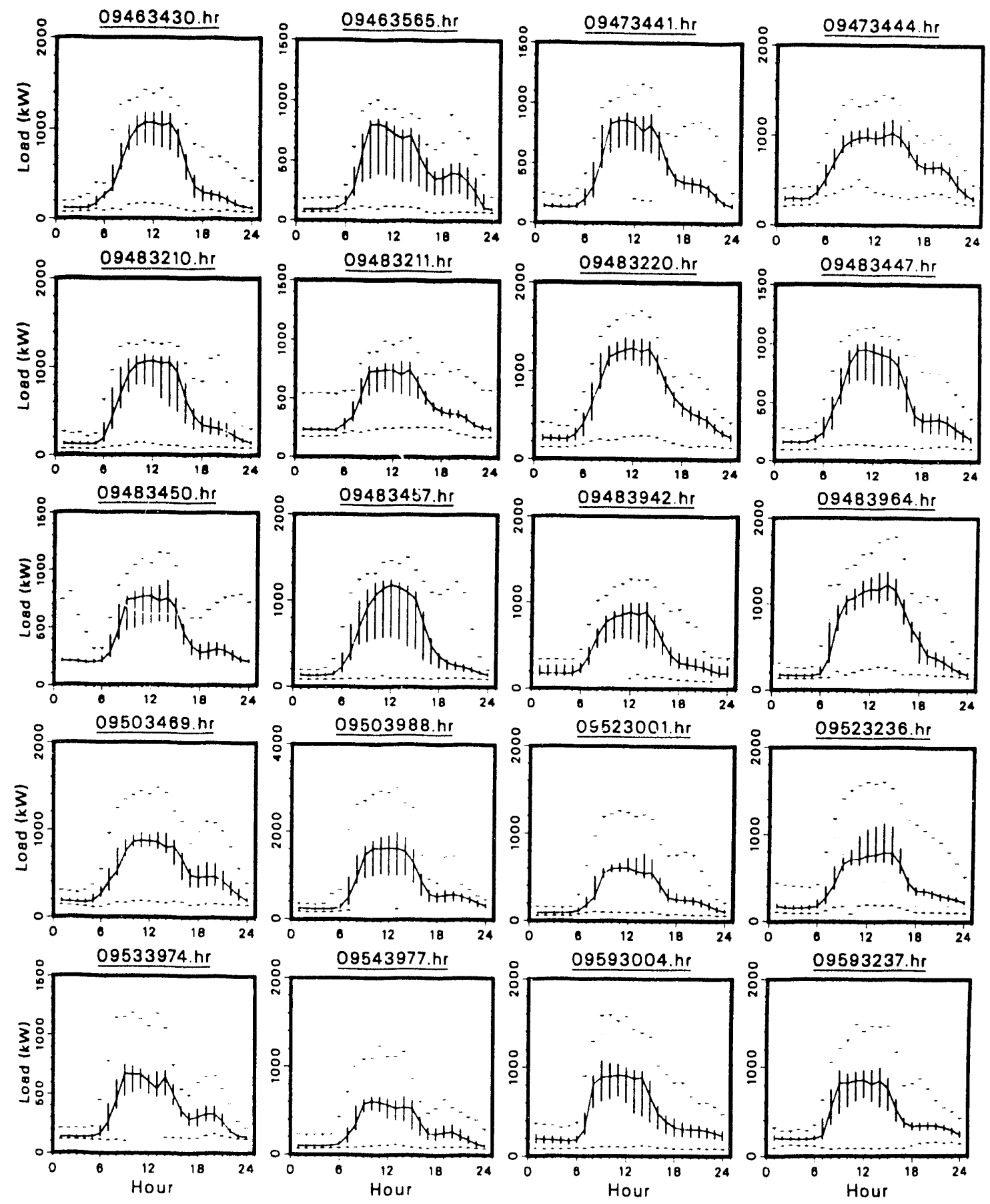
Average Weekday Profiles for Schools
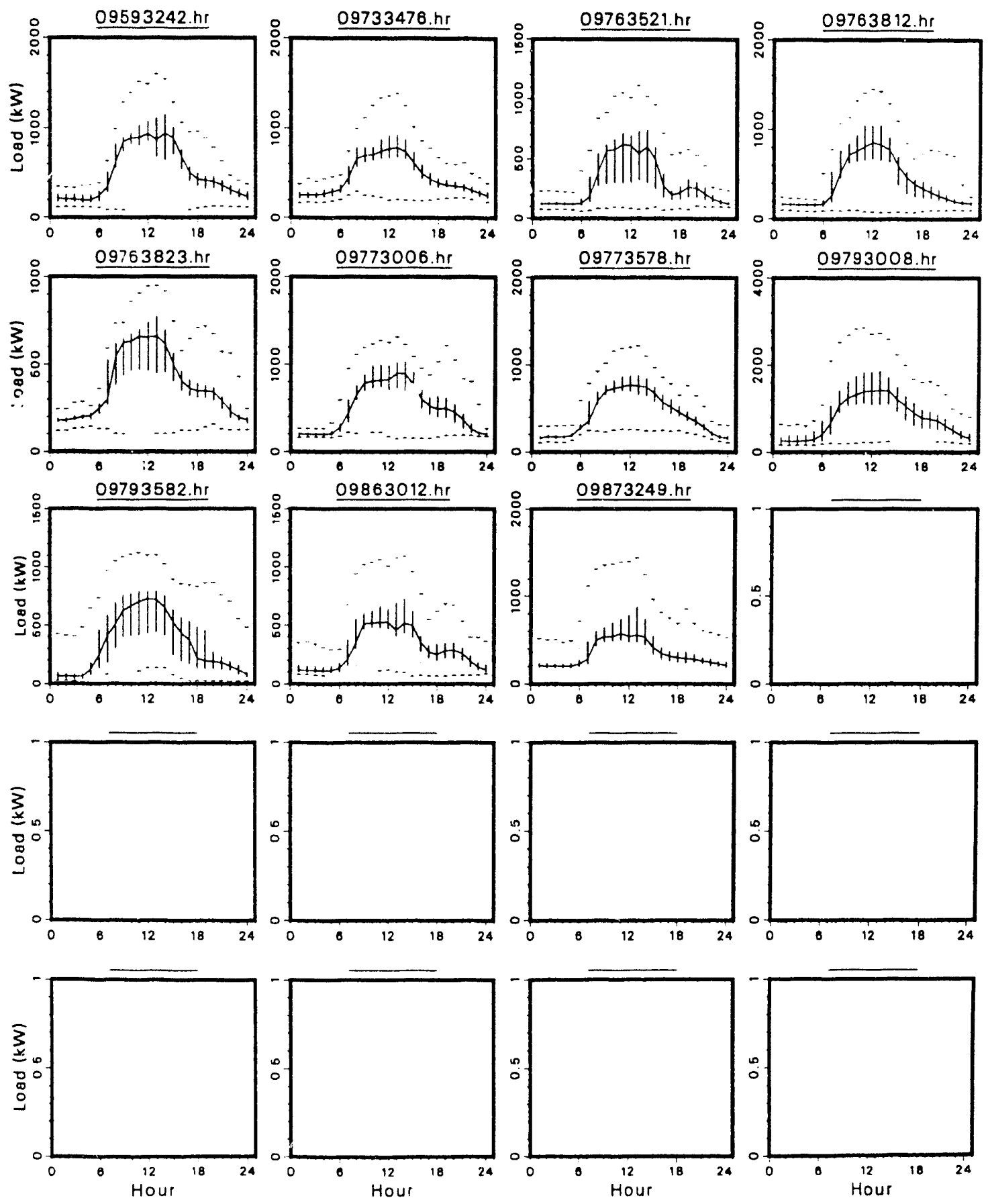
Average Weekday Profiles for Colleges
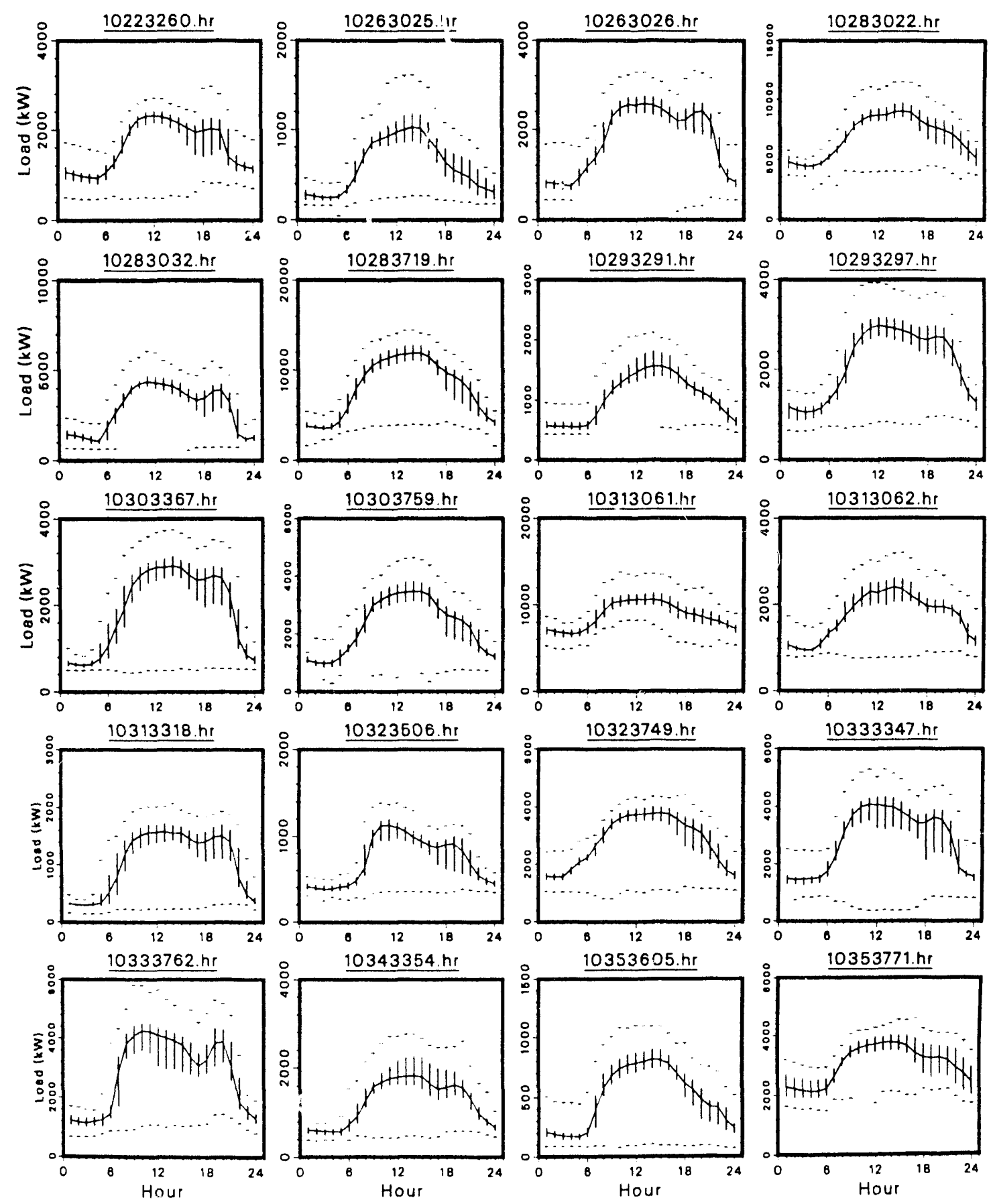


\section{Average Weekday Profiles for Colleges}
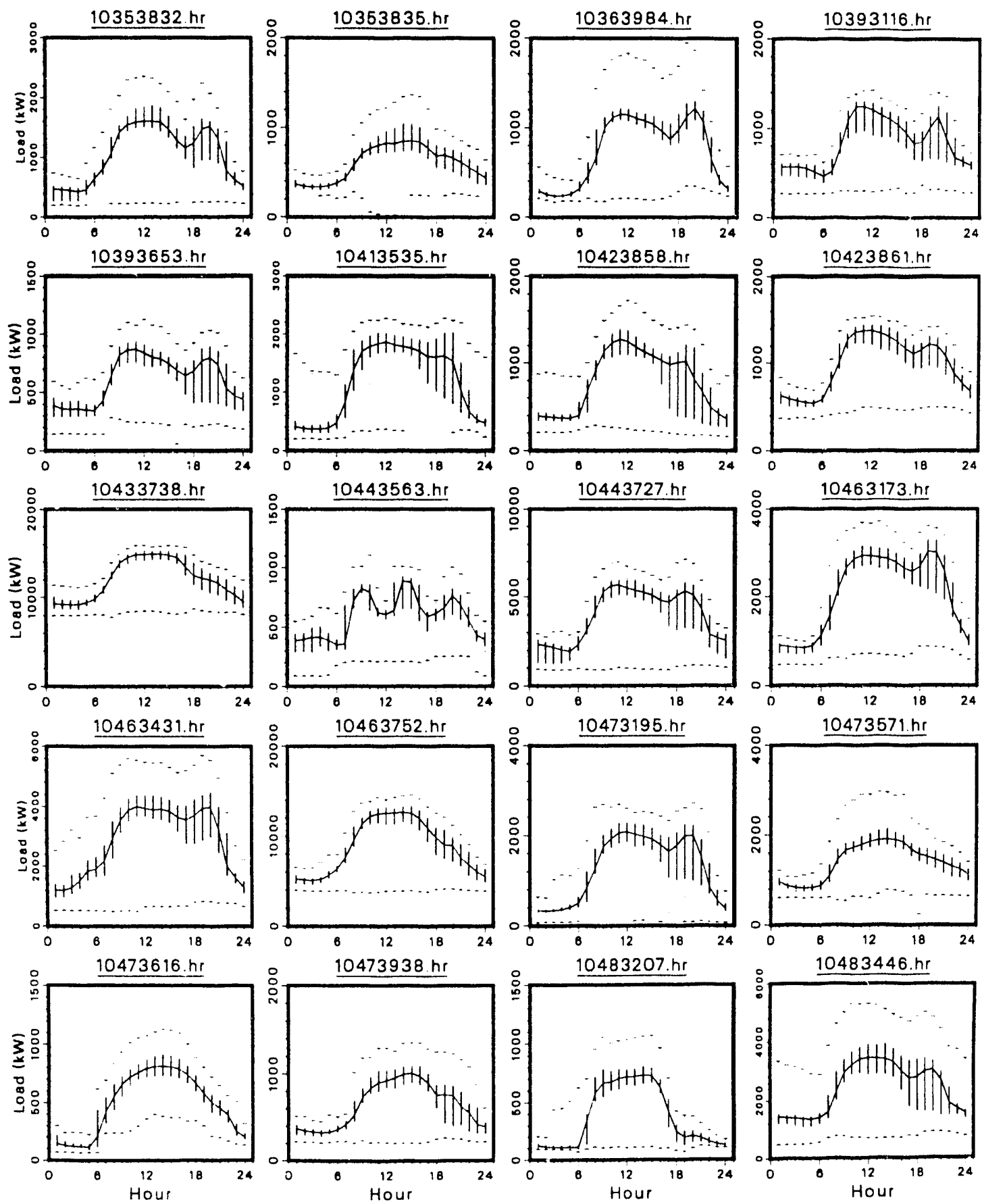


\section{Average Weekday Profiles for Colleges}
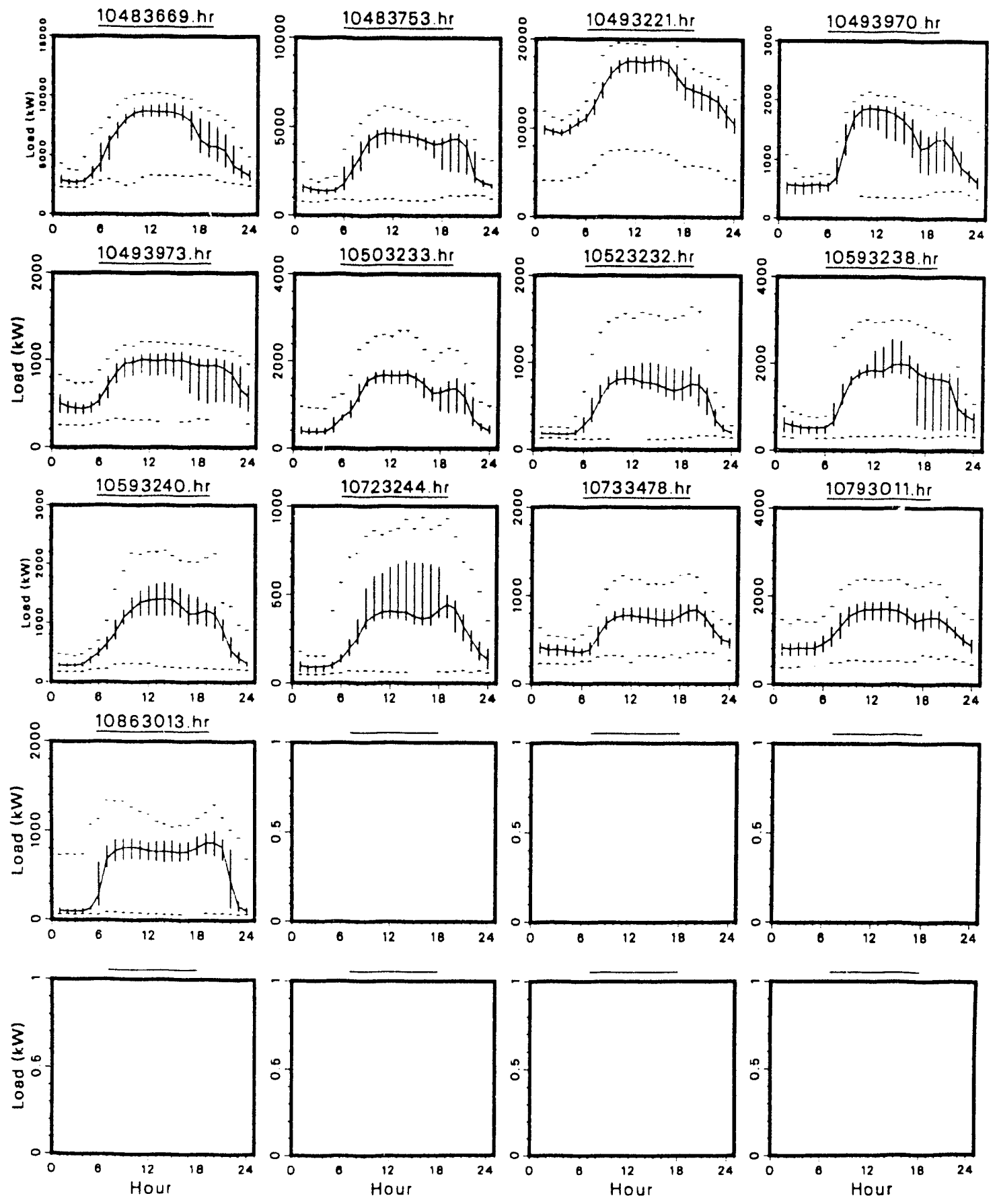


\section{Average Weekday Profiles for Hospitals}
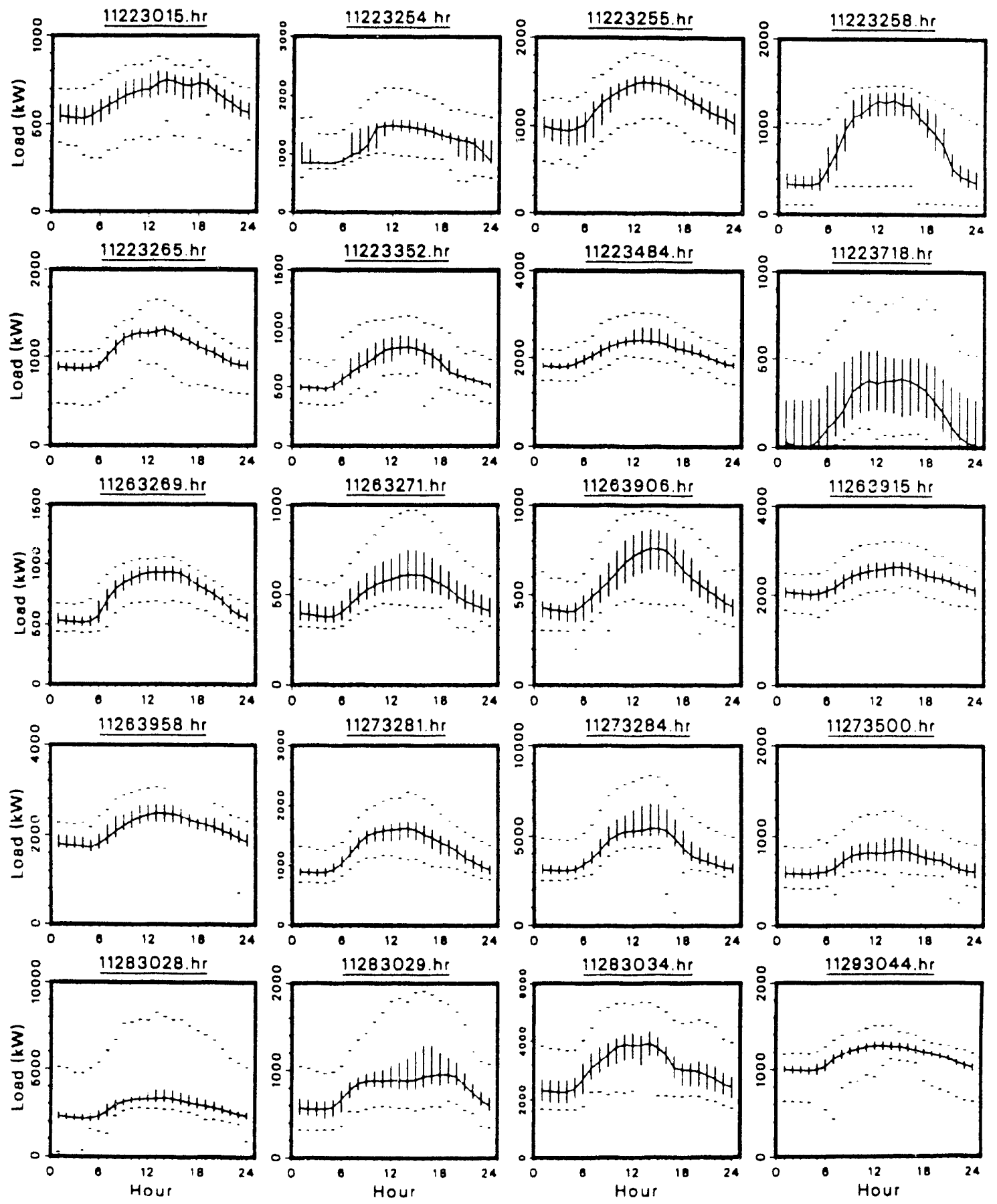


\section{Average Weekday Profiles for Hospitals}
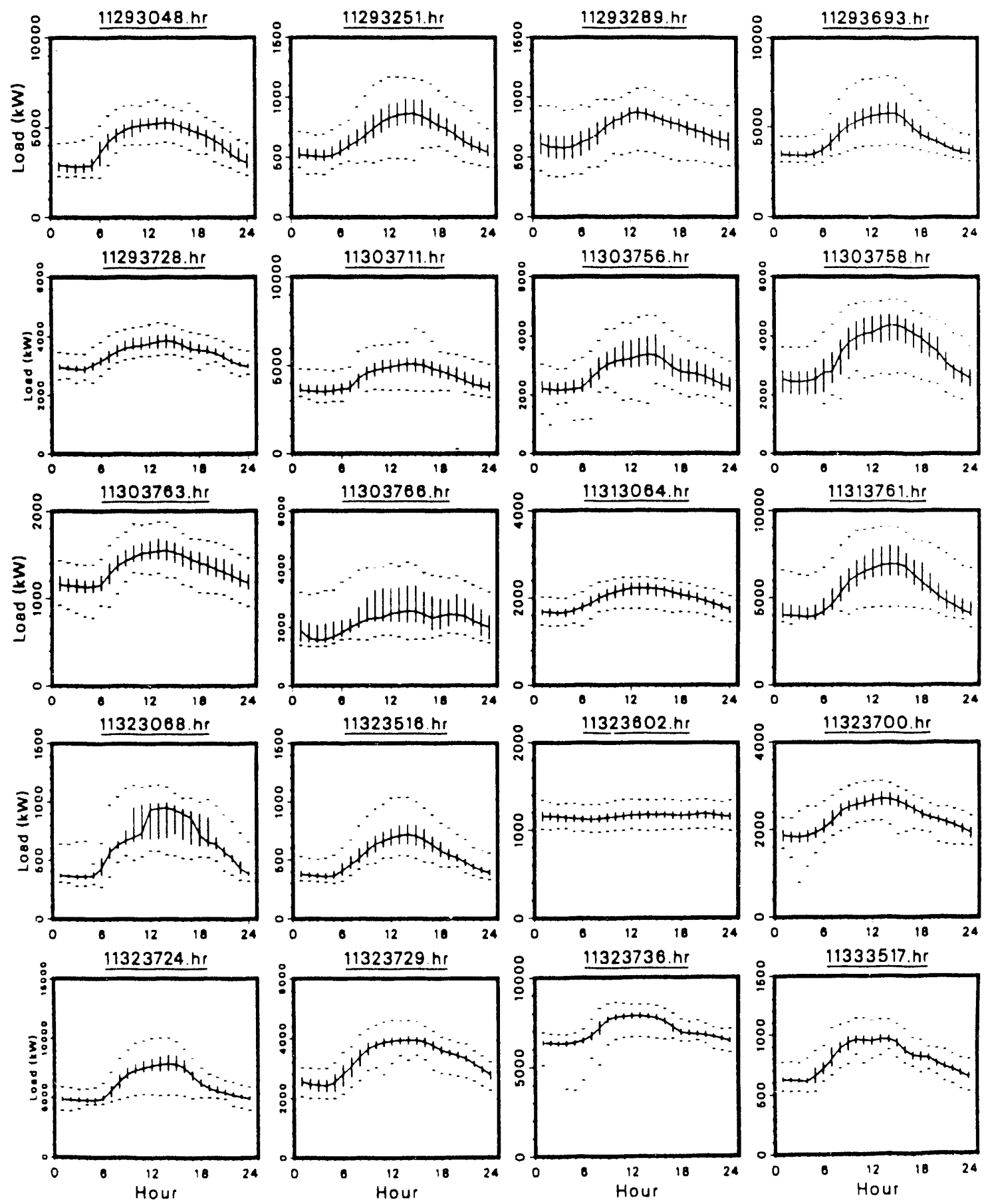
Average Weekday Profiles for Hospitals
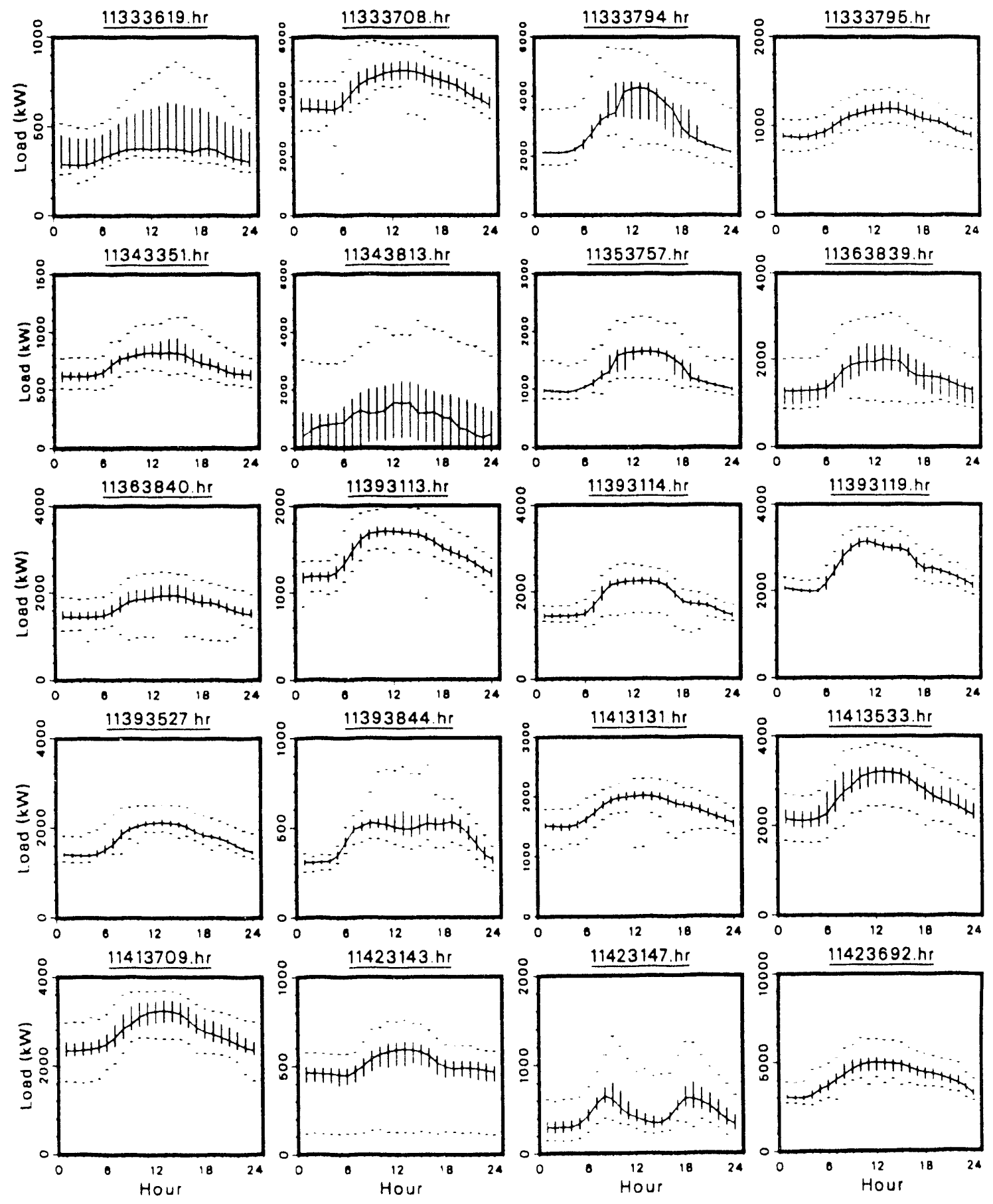


\section{Average Weekday Profiles for Hospitals}
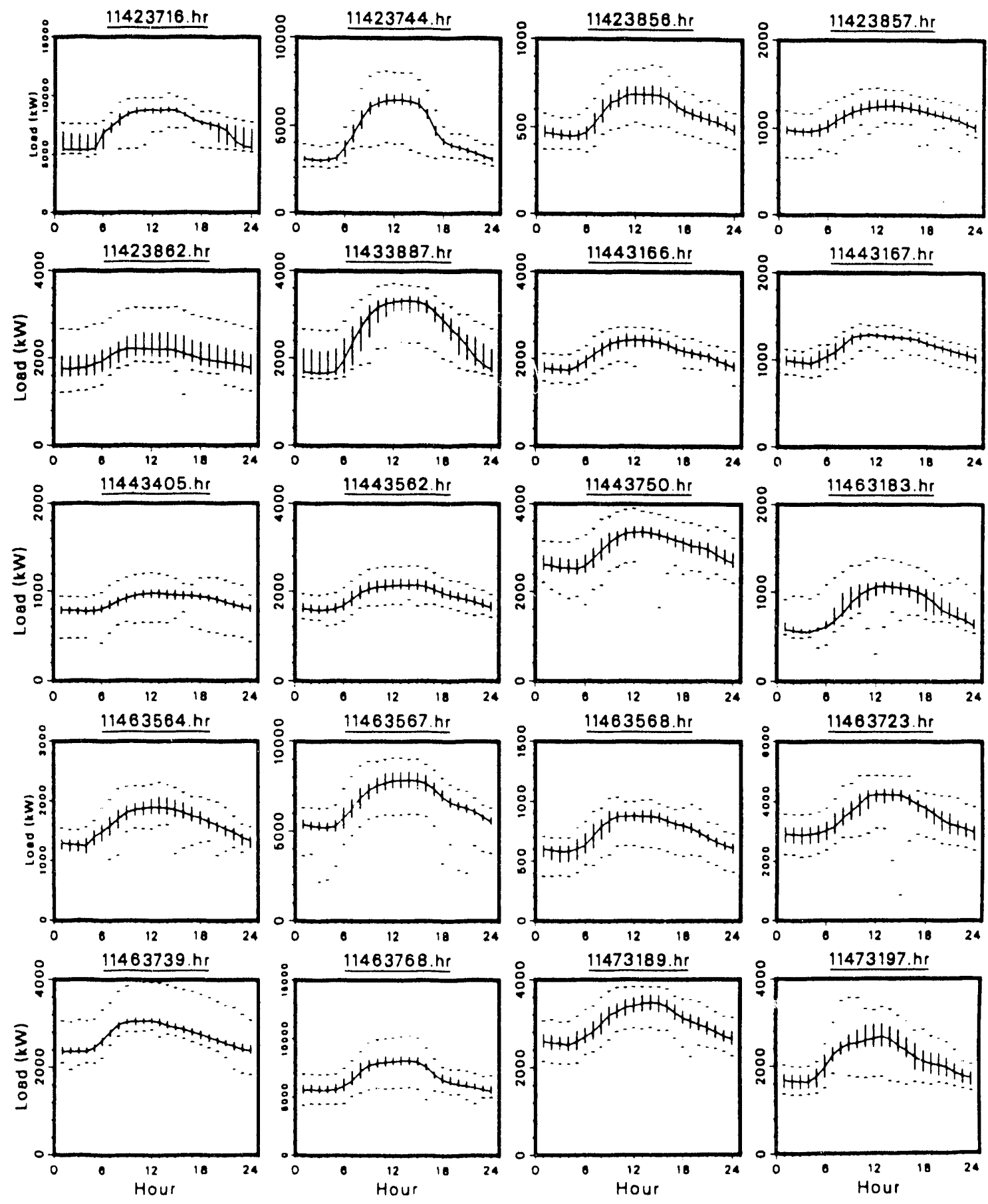


\section{Average Weekday Profiles for Hospitals}
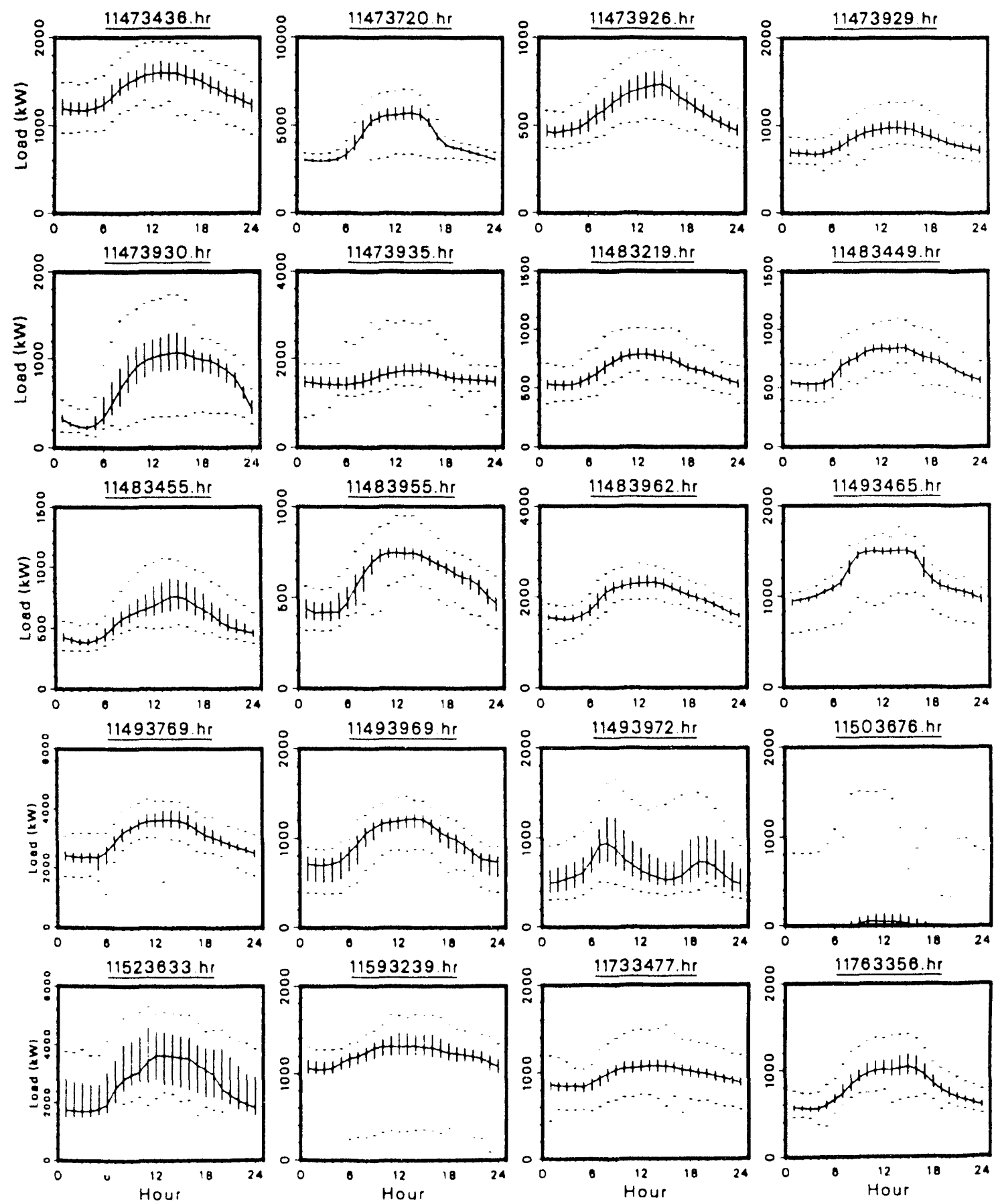


\section{Average Weekday Profiles for Hospitals (and Health)}
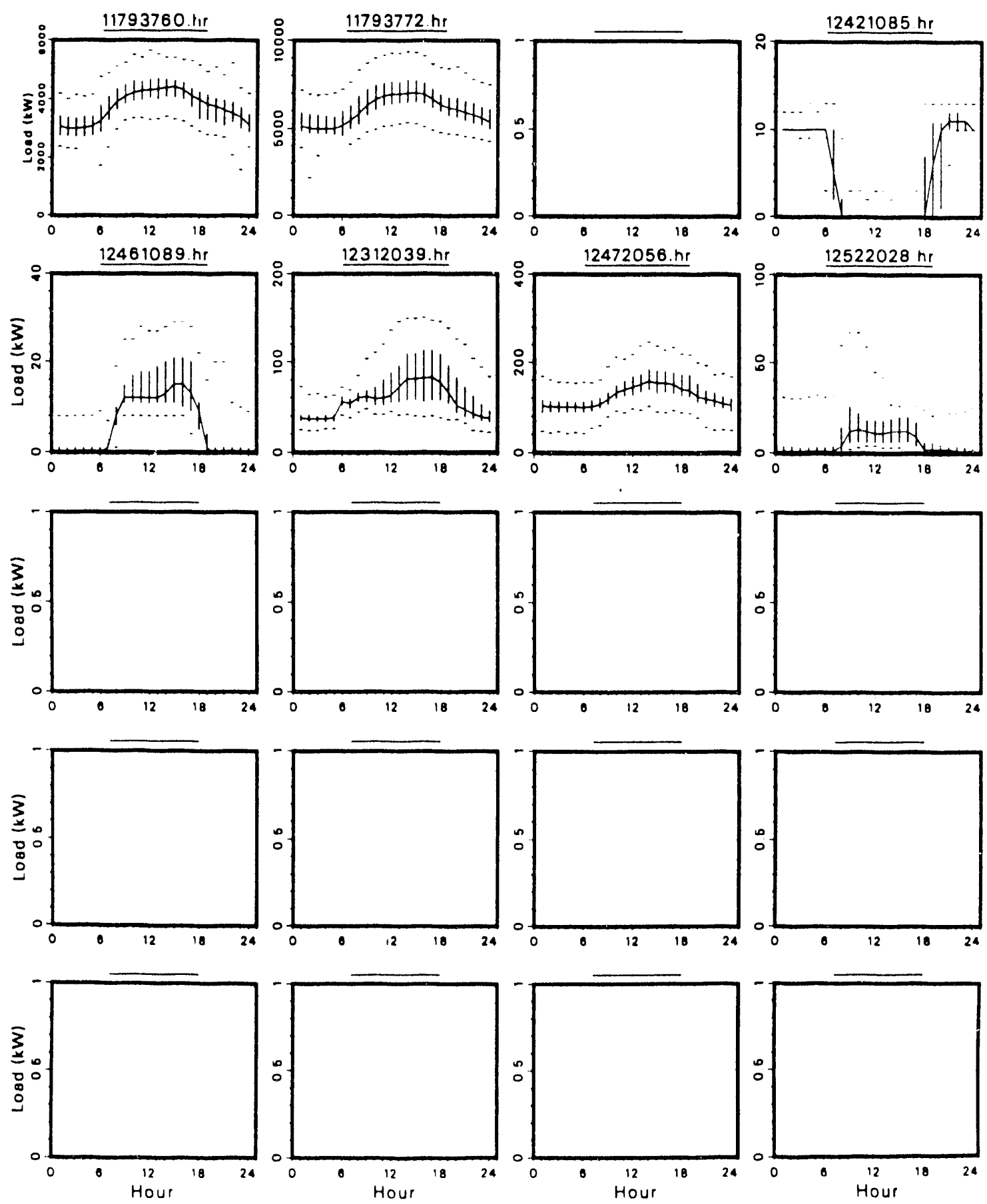
Average Weekday Profiles for Hotels \& Motels
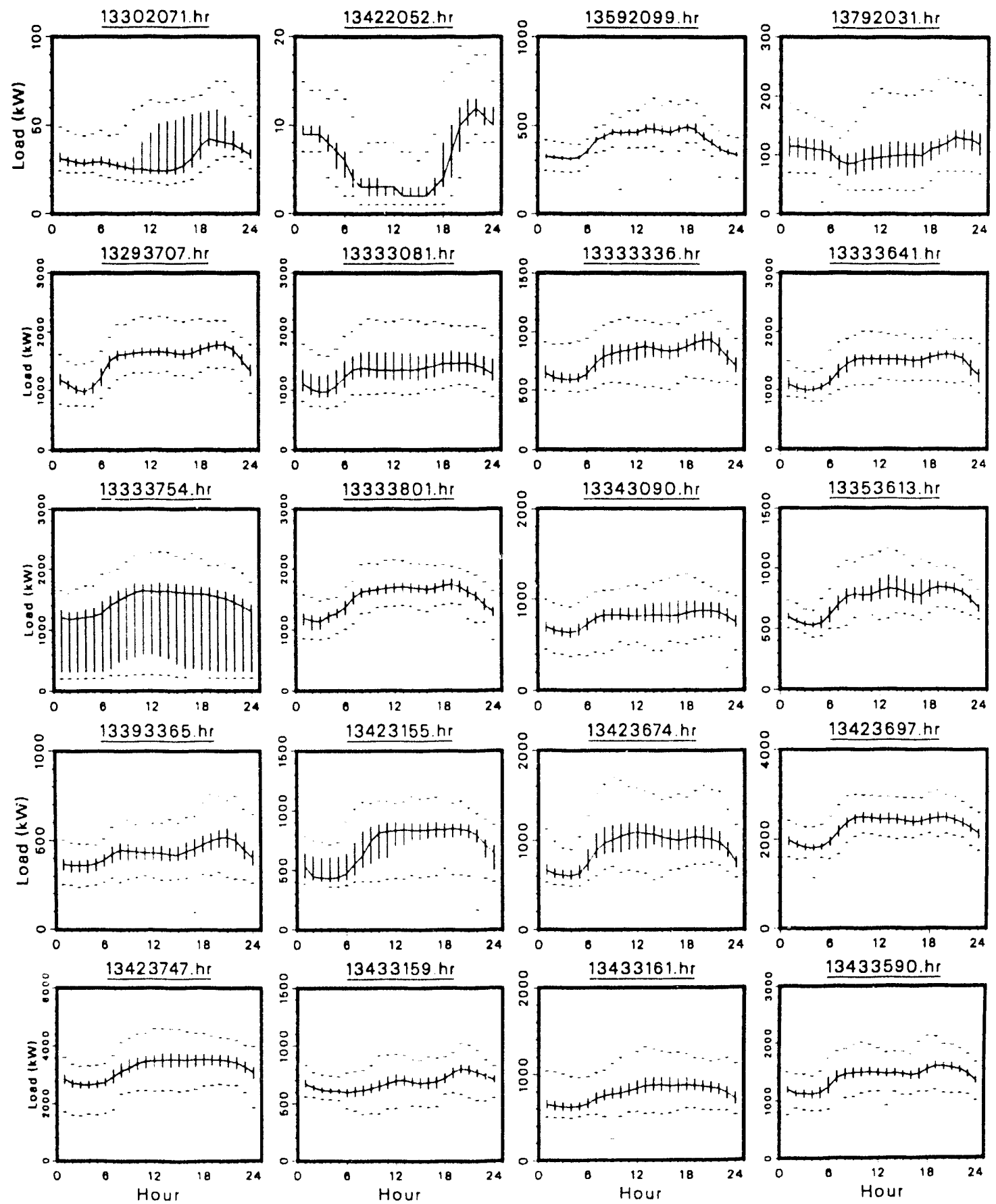
Average Weekday Profiles for Hotels \& Motels
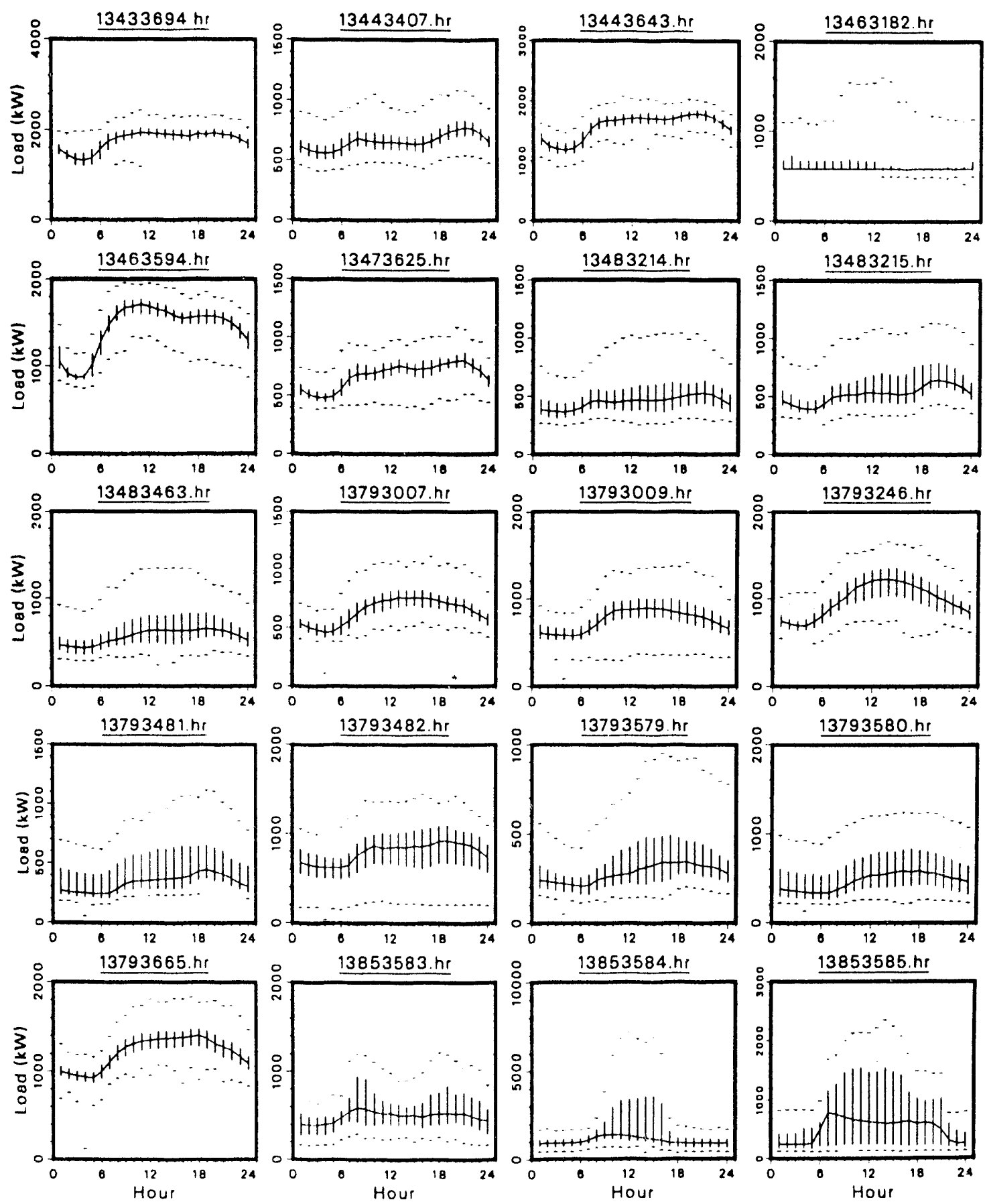
Appendix D. Results from Load-Temperature Regressions 
LRD Regression Coefficlents for Coastal Health - Standard Day - Summer

\begin{tabular}{|c|c|c|c|c|c|c|}
\hline Hour & $\begin{array}{l}\text { Regr } \\
\text { Base Load } \\
\text { Intercept } \\
\left(W / t^{2}\right)\end{array}$ & $\begin{array}{l}\text { sslon Paran } \\
\text { Dew Point } \\
\text { Coefficeint } \\
\left(W / t^{2} /{ }^{\circ} F\right)\end{array}$ & $\begin{array}{l}\text { ters } \\
\text { Dry Bulb } \\
\text { Coefficient } \\
\left(\mathrm{W} / \mathrm{ft}^{2} /{ }^{\circ} \mathrm{F}\right)\end{array}$ & $\mathrm{R}^{2}$ & $\begin{array}{c}\text { Statistics } \\
\text { Significance } \\
\text { of } \\
\text { F-Statistic }\end{array}$ & $N$ \\
\hline 1 & 2.233 & 0.009 & 0.043 & 0.79 & 0.000 & 102 \\
\hline 2 & 2.214 & 0.009 & 0.041 & 0.79 & 0.000 & 102 \\
\hline 3 & 2.215 & 0.008 & 0.042 & 0.77 & 0.000 & 98 \\
\hline 4 & 2.199 & 0.011 & 0.040 & 0.75 & 0.000 & 96 \\
\hline 5 & 2.287 & 0.009 & 0.040 & 0.73 & 0.000 & 93 \\
\hline 6 & 2.429 & 0.009 & 0.040 & 0.66 & 0.000 & 93 \\
\hline 7 & 2.590 & 0.012 & 0.046 & 0.67 & 0.000 & 96 \\
\hline 8 & 2.758 & 0.015 & 0.043 & 0.87 & 0.000 & 114 \\
\hline 9 & 2.842 & 0.023 & 0.035 & 0.85 & 0.000 & 124 \\
\hline 10 & 2.913 & 0.025 & 0.031 & 0.83 & 0.000 & 125 \\
\hline 11 & 2.940 & 0.025 & 0.033 & 0.84 & 0.000 & 126 \\
\hline 12 & 2.931 & 0.023 & 0.037 & 0.81 & 0.000 & 126 \\
\hline 13 & 2.958 & 0.022 & 0.037 & 0.76 & 0.000 & 126 \\
\hline 14 & 3.022 & 0.017 & 0.034 & 0.75 & 0.000 & 126 \\
\hline 15 & 3.004 & 0.016 & 0.031 & 0.73 & 0.000 & 126 \\
\hline 16 & 2.854 & 0.021 & 0.031 & 0.76 & 0.000 & 126 \\
\hline 17 & 2.712 & 0.020 & 0.034 & 0.78 & 0.000 & 126 \\
\hline 18 & 2.575 & 0.026 & 0.038 & 0.81 & 0.000 & 125 \\
\hline 19 & 2.554 & 0.024 & 0.040 & 0.83 & 0.000 & $1 ? 4$ \\
\hline 20 & 2.468 & 0.024 & 0.044 & 0.86 & 0.000 & $12 i$ \\
\hline 21 & 2.407 & 0.024 & 0.044 & 0.80 & 0.000 & 119 \\
\hline 22 & 2.358 & 0.020 & 0.042 & 0.80 & 0.000 & 118 \\
\hline 23 & 2.271 & 0.019 & 0.040 & 0.81 & 0.000 & 118 \\
\hline 24 & 2.208 & 0.017 & 0.041 & 0.82 & 0.000 & 115 \\
\hline
\end{tabular}

I.RD Regression Coefficients for Coastal Health - Standard Day - Winter

\begin{tabular}{|r|ccc|ccr|}
\hline Hour & $\begin{array}{c}\text { Base Load } \\
\text { Intercept } \\
\left(\text { W/ft } \mathrm{f}^{2}\right)\end{array}$ & $\begin{array}{c}\text { Regression Parameters } \\
\text { Dew Point } \\
\text { Coefficeint } \\
\left(\text { W/ft }^{2} /{ }^{\circ} \mathrm{F}\right)\end{array}$ & $\begin{array}{c}\text { Dry Bulb } \\
\text { Coefficient } \\
\left(\text { W/ft }{ }^{\circ} /{ }^{\circ} \mathrm{F}\right)\end{array}$ & $\mathrm{R}^{2}$ & $\begin{array}{c}\text { Statistics } \\
\text { Significance } \\
\text { of } \\
\text { F-Statistic }\end{array}$ & $\mathrm{N}$ \\
\hline 1 & 2.135 & 0.020 & 0.046 & 0.70 & 0.001 & 14 \\
2 & 2.156 & 0.016 & 0.032 & 0.50 & 0.086 & 9 \\
3 & 2.049 & $0.03 i$ & 0.051 & 0.84 & 0.010 & 7 \\
4 & 2.174 & 0.000 & 0.070 & 0.97 & 0.033 & 4 \\
5 & 2.320 & -0.020 & 0.050 & 0.98 & 0.017 & 4 \\
6 & 2.470 & -0.021 & 0.008 & 0.71 & 0.155 & 5 \\
7 & 2.555 & -0.006 & 0.045 & 0.87 & 0.017 & 6 \\
8 & 2.689 & 0.005 & 0.047 & 0.42 & 0.029 & 15 \\
9 & 2.903 & -0.000 & 0.024 & 0.22 & 0.013 & 37 \\
10 & 3.032 & 0.002 & 0.025 & 0.44 & 0.000 & 65 \\
11 & 3.039 & 0.002 & 0.026 & 0.60 & 0.000 & 96 \\
12 & 3.017 & 0.006 & 0.032 & 0.71 & 0.000 & 112 \\
13 & 2.995 & 0.008 & 0.033 & 0.71 & 0.000 & 116 \\
14 & 3.034 & 0.009 & 0.032 & 0.68 & 0.000 & 115 \\
15 & 2.980 & 0.008 & 0.032 & 0.66 & 0.000 & 115 \\
16 & 2.945 & 0.007 & 0.030 & 0.59 & 0.000 & 116 \\
17 & 2.816 & 0.007 & 0.031 & 0.64 & 0.000 & 117 \\
18 & 2.734 & 0.005 & 0.030 & 0.58 & 0.000 & 105 \\
19 & 2.696 & 0.005 & 0.031 & 0.50 & 0.000 & 79 \\
20 & 2.603 & 0.004 & 0.038 & 0.60 & 0.000 & 63 \\
21 & 2.552 & 0.004 & 0.032 & 0.53 & 0.000 & 43 \\
22 & 2.484 & 0.003 & 0.034 & 0.49 & 0.000 & 29 \\
23 & 2.370 & 0.005 & 0.037 & 0.53 & 0.001 & 21 \\
24 & 2.250 & 0.007 & 0.061 & 0.69 & 0.000 & 16 \\
\hline
\end{tabular}


LRD Regression Coefficients for Coastal Health - Non-Standard Day - Summer

\begin{tabular}{|c|ccc|ccc|}
\hline Hour & $\begin{array}{c}\text { Base Load } \\
\text { Intercept } \\
\left(\text { W/t } / \mathrm{t}^{2}\right)\end{array}$ & $\begin{array}{c}\text { Regression Parameters } \\
\text { Dew Point } \\
\text { Coefficeint } \\
\left(\text { W/ft }{ }^{2} /{ }^{\circ} \mathrm{F}\right)\end{array}$ & $\begin{array}{c}\text { Dry Bulb } \\
\text { Coefficient } \\
\left(\text { W/ft }{ }^{2} /{ }^{\circ} \mathrm{F}\right)\end{array}$ & $\mathrm{R}^{2}$ & $\begin{array}{c}\text { Statistics } \\
\text { Significance } \\
\text { of } \\
\text { F-Statistic }\end{array}$ & $\mathrm{N}$ \\
\hline 1 & 2.182 & 0.008 & 0.051 & 0.77 & 0.000 & 47 \\
2 & 2.183 & 0.005 & 0.053 & 0.78 & 0.000 & 44 \\
3 & 2.229 & 0.003 & 0.043 & 0.64 & 0.000 & 41 \\
4 & 2.184 & 0.010 & 0.043 & 0.69 & 0.000 & 39 \\
5 & 2.243 & 0.011 & 0.038 & 0.62 & 0.000 & 36 \\
6 & 2.255 & 0.016 & 0.038 & 0.68 & 0.000 & 37 \\
7 & 2.328 & 0.015 & 0.037 & 0.63 & 0.000 & 40 \\
8 & 2.384 & 0.008 & 0.043 & 0.66 & 0.000 & 47 \\
9 & 2.439 & 0.015 & 0.030 & 0.66 & 0.000 & 55 \\
10 & 2.500 & 0.019 & 0.026 & 0.57 & 0.000 & 55 \\
11 & 2.421 & 0.022 & 0.032 & 0.62 & 0.000 & 55 \\
12 & 2.528 & 0.019 & 0.029 & 0.52 & 0.000 & 55 \\
13 & 2.496 & 0.017 & 0.034 & 0.55 & 0.000 & 55 \\
14 & 2.548 & 0.018 & 0.034 & 0.53 & 0.000 & 55 \\
15 & 2.540 & 0.015 & 0.031 & 0.58 & 0.000 & 55 \\
16 & 2.521 & 0.020 & 0.028 & 0.65 & 0.000 & 55 \\
17 & 2.523 & 0.019 & 0.028 & 0.67 & 0.000 & 55 \\
18 & 2.422 & 0.020 & 0.035 & 0.77 & 0.000 & 55 \\
19 & 2.429 & 0.021 & 0.037 & 0.82 & 0.000 & 55 \\
20 & 2.306 & 0.024 & 0.044 & 0.83 & 0.000 & 55 \\
21 & 2.246 & 0.025 & 0.047 & 0.80 & 0.000 & 53 \\
22 & 2.221 & 0.020 & 0.046 & 0.64 & 0.000 & 52 \\
23 & 2.230 & 0.010 & 0.048 & 0.66 & 0.000 & 52 \\
24 & 2.136 & 0.013 & 0.051 & 0.78 & 0.000 & 50 \\
\hline
\end{tabular}

LRD Regression Coefficients for Coastal Health - Non-Standard Day - Winter

\begin{tabular}{|c|c|c|c|c|c|c|}
\hline Hour & $\begin{array}{l}\text { Reg } \\
\text { Base Load } \\
\text { Intercept } \\
\left(W / t^{2}\right)\end{array}$ & $\begin{array}{l}\text { ssion Paran } \\
\text { Dew Point } \\
\text { Coefticeint } \\
\left(W^{\prime} \mathrm{ft}^{2} /{ }^{\circ} \mathrm{F}\right)\end{array}$ & $\begin{array}{l}\text { ters } \\
\text { Dry Bulb } \\
\text { Coefficient } \\
\left(\mathrm{W} / \mathrm{ft}^{2} /{ }^{\circ} \mathrm{F}\right)\end{array}$ & $\mathrm{R}^{2}$ & $\begin{array}{c}\text { Statistics } \\
\text { Significance } \\
\text { of } \\
\text { F-Statistic }\end{array}$ & $N$ \\
\hline 1 & 2.199 & 0.010 & 0.024 & 0.95 & 0.010 & 5 \\
\hline 2 & 15.835 & 0.006 & 0.259 & 1.00 & 2.000 & 0 \\
\hline 3 & 0.000 & 0.000 & 0.000 & 0.00 & 1.000 & 0 \\
\hline 4 & 0.000 & 0.000 & 0.000 & 0.00 & 1.000 & 0 \\
\hline 5 & 0.000 & 0.000 & 0.000 & 0.00 & 1.000 & 0 \\
\hline 6 & 0.000 & 0.000 & 0.000 & 0.00 & 1.000 & 0 \\
\hline 7 & 0.000 & 0.000 & 0.000 & 0.00 & 1.000 & 0 \\
\hline 8 & 2.370 & 0.003 & 0.027 & 0.66 & 0.342 & 4 \\
\hline 9 & 2.579 & -0.010 & -0.008 & 0.54 & 0.045 & 10 \\
\hline 10 & 2.556 & -0.005 & 0.018 & 0.54 & 0.000 & 32 \\
\hline 11 & 2.525 & -0.003 & 0.021 & 0.64 & 0.000 & 42 \\
\hline 12 & 2.470 & 0.003 & 0.028 & 0.72 & 0.000 & 51 \\
\hline 13 & 2.462 & 0.003 & 0.027 & 0.75 & 0.000 & 52 \\
\hline 14 & 2.493 & 0.005 & 0.028 & 0.76 & 0.00 & 52 \\
\hline 15 & 2.446 & 0.005 & 0.030 & 0.80 & 0.00 & 54 \\
\hline 16 & 2.432 & 0.006 & 0.030 & 0.75 & 0.000 & 51 \\
\hline 17 & 2.410 & 0.004 & 0.030 & 0.73 & 0.000 & 49 \\
\hline 18 & 2.449 & 0.001 & 0.026 & 0.73 & 0.000 & 47 \\
\hline 19 & 2.482 & 0.004 & 0.031 & 0.72 & 0.000 & 39 \\
\hline 20 & 2.468 & 0.002 & 0.023 & 0.66 & 0.000 & 31 \\
\hline 21 & 2.402 & 0.000 & 0.022 & 0.68 & 0.000 & 25 \\
\hline 22 & 2.342 & 0001 & 0.020 & 0.45 & 0.002 & 23 \\
\hline 23 & 2.319 & 0.001 & 0.012 & 0.41 & 0.053 & 13 \\
\hline 24 & 2.262 & 0.002 & 0.006 & 0.23 & 0.352 & 10 \\
\hline
\end{tabular}


LRD Regression Coefficients for Non-Coastal Health - Standard Day - Summer

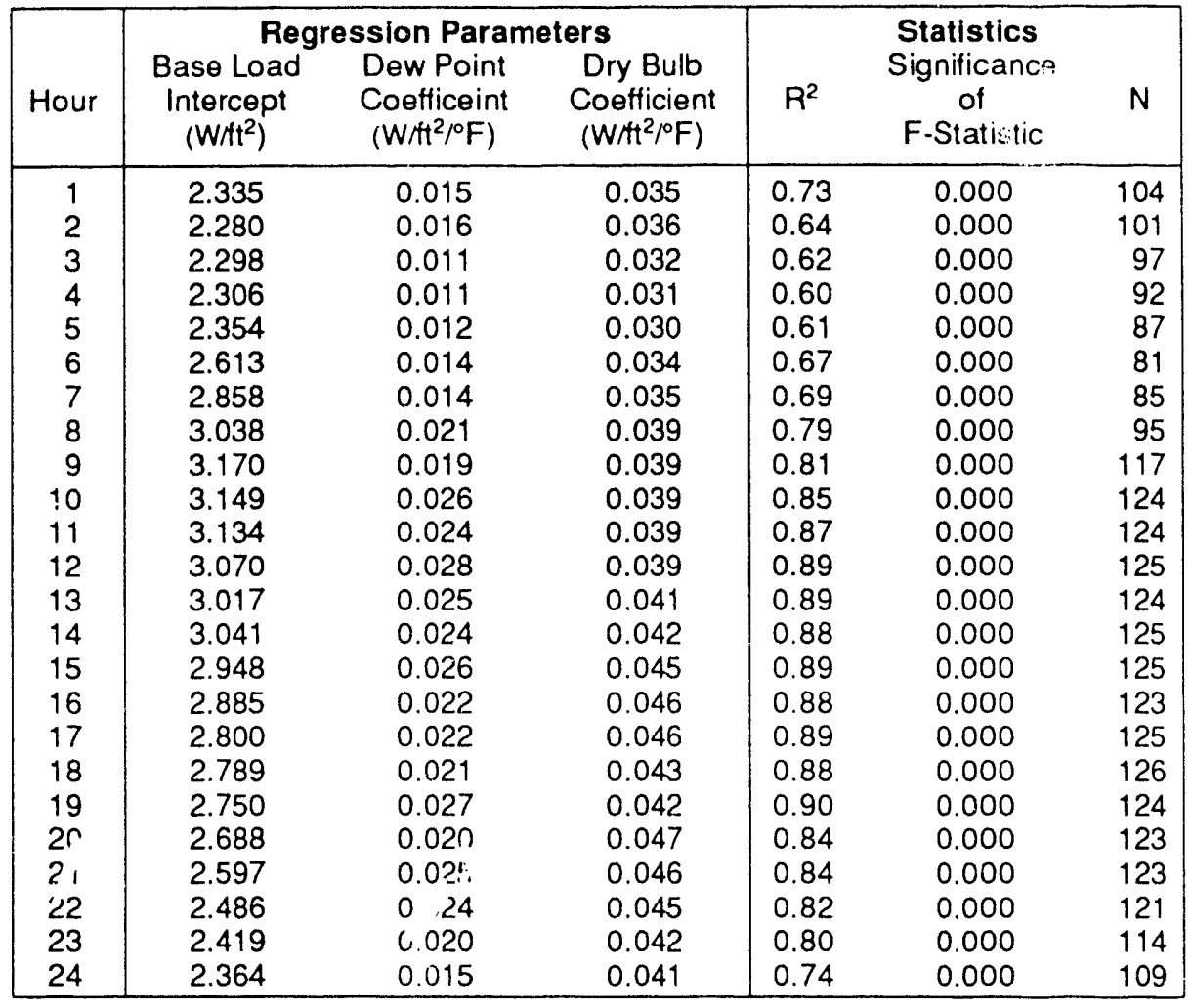

LRD Regression Coefficients for Non-Coastal Health - Standard Day - Winter

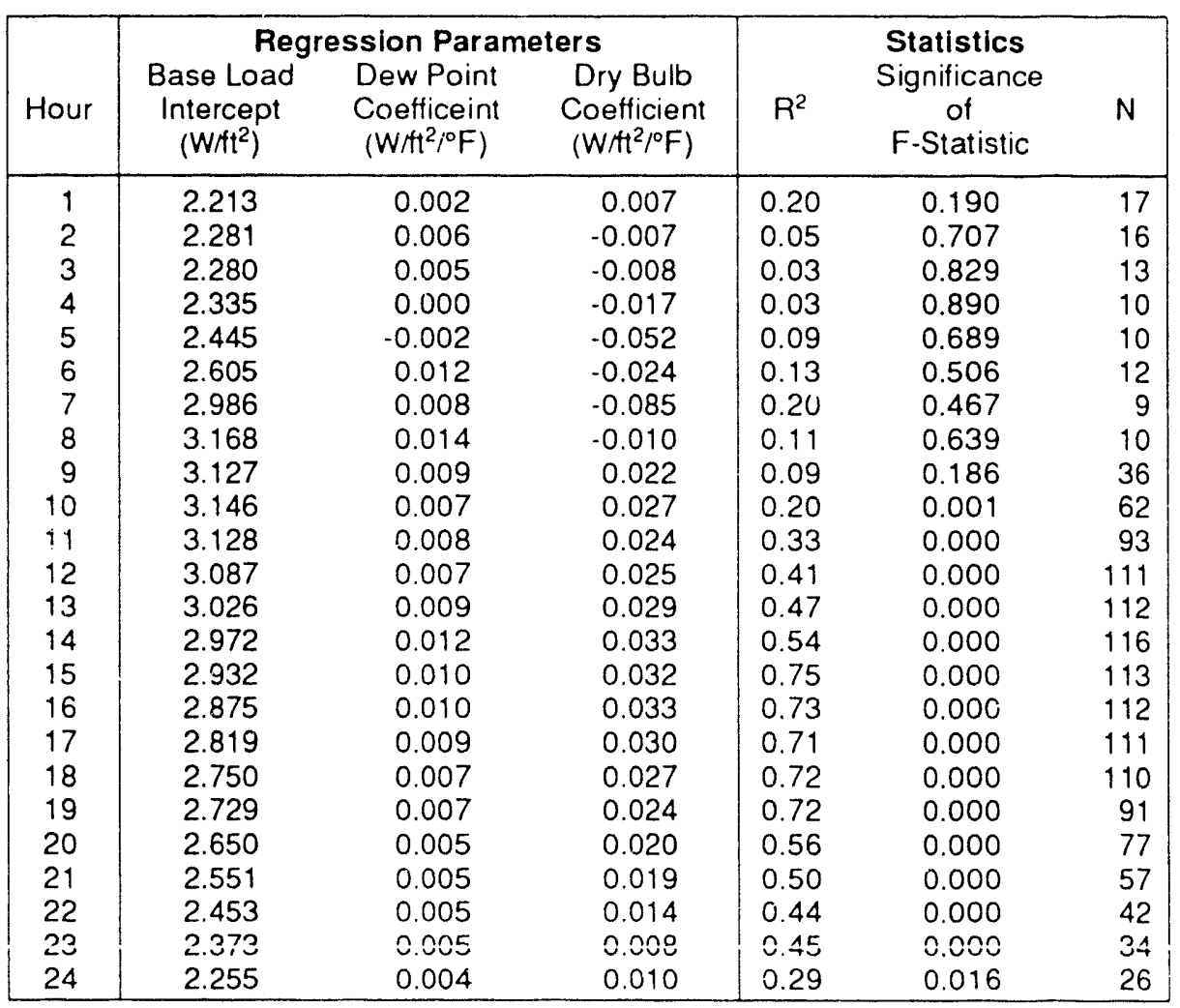


LRD Regression Coefficlents for Non-Coastal Health - Non-Standard Day - Summer

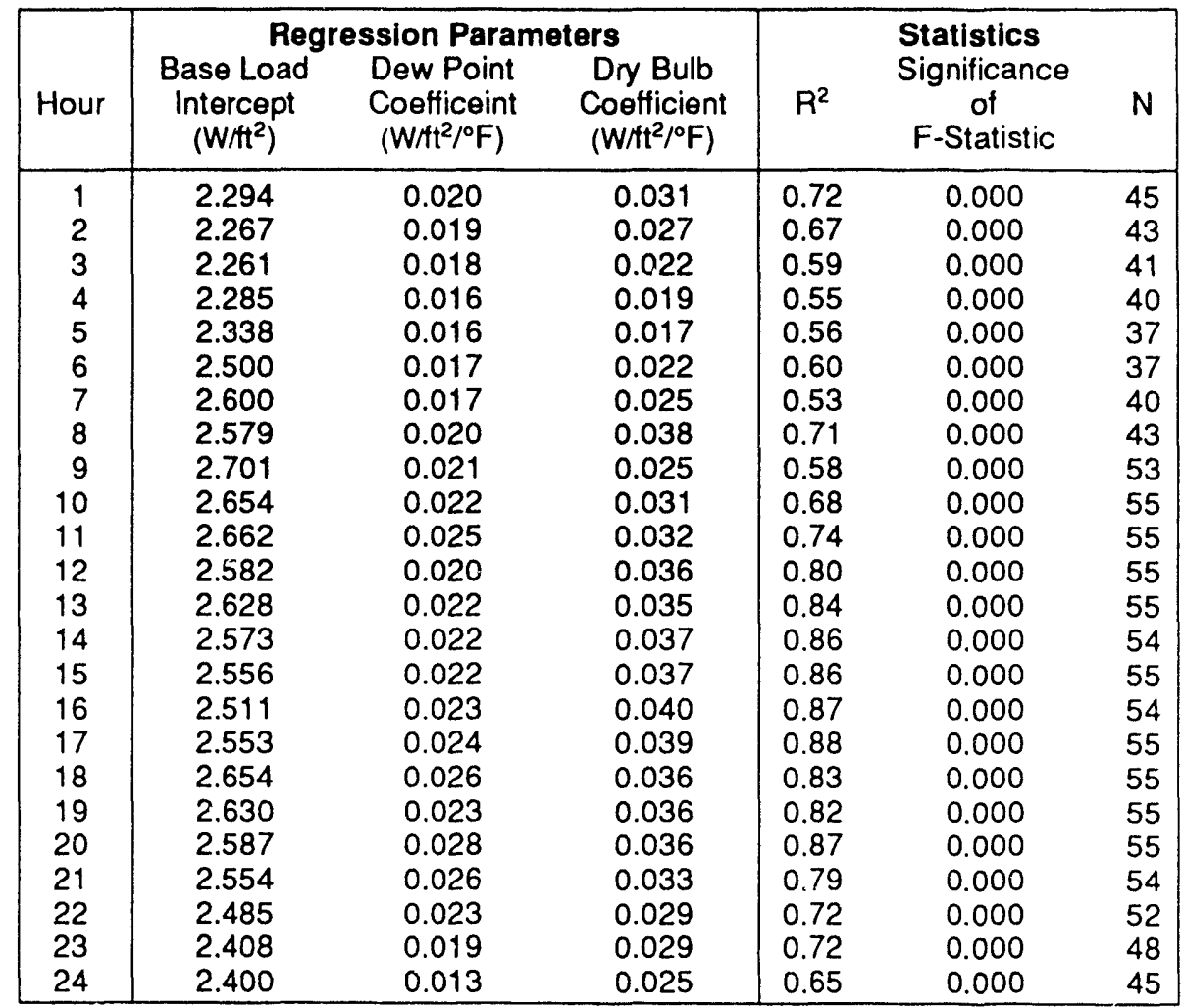

LRD Regression Coefficients for Non-Coastal Health - Non-Standard Day - Winter

\begin{tabular}{|c|c|c|c|c|c|c|}
\hline Hour & $\begin{array}{r}\text { Peg } \\
\text { Base Load } \\
\text { Intercept } \\
\left(\mathrm{W} / \mathrm{tt}^{2}\right)\end{array}$ & $\begin{array}{l}\text { ssion Parar } \\
\text { Dew Point } \\
\text { Coefficeint } \\
\left(\mathrm{W} / \mathrm{t}^{2} /{ }^{\circ} \mathrm{F}\right)\end{array}$ & $\begin{array}{l}\text { Drs } \\
\text { Dry Bulb } \\
\text { Coefficient } \\
\left(\mathrm{W}^{\prime} t \mathrm{t}^{2} /{ }^{\circ} \mathrm{F}\right)\end{array}$ & $R^{2}$ & $\begin{array}{c}\text { Statistics } \\
\text { Significance } \\
\text { of } \\
\text { F-Statistic }\end{array}$ & $N$ \\
\hline 1 & 2.193 & 0.002 & 0.006 & 0.07 & 0.746 & 10 \\
\hline 2 & 2.033 & -0.008 & 0.040 & 0.44 & 0.745 & 3 \\
\hline 3 & 4.539 & -0.000 & 3.040 & 0.00 & 0.952 & 4 \\
\hline 4 & 4.410 & -0.007 & 0.040 & 0.55 & 0.258 & 4 \\
\hline 5 & 4.382 & -0.009 & 0.040 & 0.69 & 0.171 & 4 \\
\hline 6 & 2.018 & -0.015 & 0.041 & 0.83 & 0.167 & 4 \\
\hline 7 & 2.020 & -0.017 & -0.004 & 0.94 & 0.243 & 3 \\
\hline 8 & 2.613 & 0.005 & -0.005 & 0.20 & 0.647 & 6 \\
\hline 9 & 2.597 & -0.002 & 0.003 & 0.06 & 0.677 & 14 \\
\hline 10 & 2.566 & 0.004 & 0.021 & 0.52 & 0.000 & 28 \\
\hline 11 & 2.500 & 0.001 & 0.022 & 0.55 & 0.000 & 36 \\
\hline 12 & 2.522 & 0.003 & 0.023 & 0.68 & 0.000 & 43 \\
\hline 13 & 2.526 & 0.005 & 0.025 & 0.71 & 0.000 & 47 \\
\hline 14 & 2.490 & 0.004 & 0.025 & C.67 & 0.000 & 48 \\
\hline 15 & 2.448 & 0.004 & 0.027 & 0.69 & 0.000 & 49 \\
\hline 16 & 2.388 & 0.006 & 0.030 & 0.77 & 0.000 & 48 \\
\hline 17 & 2.417 & 0.005 & 0.027 & 0.74 & 0.000 & 49 \\
\hline 18 & 2.508 & 0.003 & 0.023 & 0.73 & 0.000 & 47 \\
\hline 19 & 2.533 & 0.004 & 0.021 & 0.76 & 0.000 & 41 \\
\hline 20 & 2.492 & 0.001 & 0.015 & 0.36 & 0.002 & 31 \\
\hline 21 & 2.381 & -0.001 & 0.012 & 0.30 & 0.013 & 26 \\
\hline 22 & 2.322 & -0.002 & 0.005 & 0.12 & 0.273 & 22 \\
\hline 23 & 2.246 & -0.002 & 0.004 & 0.17 & 0.170 & 21 \\
\hline 24 & 2.172 & -0.001 & 0.005 & 0.12 & 0.380 & 17 \\
\hline
\end{tabular}


LRD Regression Coefficients for Coastal Lodging - Standard Day - Summer

\begin{tabular}{|c|c|c|c|c|c|c|}
\hline \multirow[b]{2}{*}{ Hour } & \multicolumn{3}{|c|}{ Regression Parameters } & \multicolumn{3}{|c|}{ Statistics } \\
\hline & $\begin{array}{l}\text { Base Load } \\
\text { Intercept } \\
\left(W / \mathrm{ft}^{2}\right)\end{array}$ & $\begin{array}{l}\text { Dew Point } \\
\text { Coefficeint } \\
\left(W / t^{2} \mu F\right)\end{array}$ & $\begin{array}{l}\text { Dry Bulb } \\
\text { Coefficient } \\
\left(W / t^{2} / F\right)\end{array}$ & $\mathrm{R}^{2}$ & $\begin{array}{c}\text { Significance } \\
\text { of } \\
\text { F-Statistic }\end{array}$ & $N$ \\
\hline 1 & 0.925 & -0.000 & 0.013 & 0.34 & 0.000 & 150 \\
\hline 2 & 0.870 & 0.000 & 0.013 & 0.42 & 0.000 & 147 \\
\hline 3 & 0.855 & 0.000 & 0.012 & 0.44 & 0.000 & 140 \\
\hline 4 & 0.854 & 0.001 & 0.012 & 0.46 & 0.000 & 136 \\
\hline 5 & 0.892 & 0.002 & 0.012 & 0.40 & 0.000 & 130 \\
\hline 6 & 0.970 & 0.004 & 0.013 & 0.32 & 0.000 & 131 \\
\hline 7 & 1.052 & 0.005 & 0.013 & 0.25 & 0.000 & 137 \\
\hline 8 & 1.095 & 0.006 & 0.012 & 0.47 & 0.000 & 162 \\
\hline 9 & 1.097 & 0.007 & 0.011 & 0.62 & 0.000 & 180 \\
\hline 10 & 1.092 & 0.007 & 0.011 & 0.67 & 0.000 & 181 \\
\hline 11 & 1.092 & 0.008 & 0.010 & 0.66 & 0.000 & 182 \\
\hline 12 & 1.082 & 0.007 & 0.010 & 0.64 & 0.000 & 182 \\
\hline 13 & 1.078 & 0.006 & 0.011 & 0.61 & 0.000 & 182 \\
\hline 14 & 1.084 & 0.005 & 0.010 & 0.57 & 0.000 & 182 \\
\hline 15 & 1.076 & 0.005 & 0.009 & 0.58 & 0.000 & 182 \\
\hline 16 & 1.074 & 0.005 & 0.009 & 0.54 & 0.000 & 182 \\
\hline 17 & 1.078 & 0.005 & 0.010 & 0.51 & 0.000 & 182 \\
\hline 18 & 1.098 & 0.006 & 0.010 & 0.40 & 0.000 & 181 \\
\hline 19 & 1.143 & 0.006 & 0.009 & 0.30 & 0.000 & 180 \\
\hline 20 & 1.153 & 0.006 & 0.012 & 0.43 & 0.000 & 180 \\
\hline 21 & 1.162 & 0.005 & 0.014 & 0.40 & 0.000 & 173 \\
\hline 22 & 1.128 & 0.004 & 0.014 & 0.40 & 0.000 & 171 \\
\hline 23 & 1.060 & 0.002 & 0.014 & 0.38 & 0.000 & 171 \\
\hline 24 & 0.991 & 0.001 & 0.013 & 0.34 & 0.000 & 166 \\
\hline
\end{tabular}

LRD Regression Coefficients for Coastal Lodging - Standard Day - Winter

\begin{tabular}{|c|c|c|c|c|c|c|}
\hline \multirow[b]{2}{*}{ Hour } & \multicolumn{3}{|c|}{ Regression Parameters } & \multicolumn{3}{|c|}{ Statistics } \\
\hline & $\begin{array}{c}\text { Base Load } \\
\text { Intercept } \\
\left(W / \mathrm{ft}^{2}\right)\end{array}$ & $\begin{array}{l}\text { Dew Point } \\
\text { Coefficeint } \\
\left(W / t^{2} /{ }^{\circ} \mathrm{F}\right)\end{array}$ & $\begin{array}{l}\text { Dry Bulb } \\
\text { Coefficient } \\
\left(\text { W/ft }{ }^{2} /{ }^{\circ} \mathrm{F}\right)\end{array}$ & $R^{2}$ & $\begin{array}{c}\text { Significance } \\
\text { of } \\
\text { F-Statistic }\end{array}$ & $N$ \\
\hline 1 & 0.906 & 0.003 & 0.006 & 0.07 & 0.516 & 20 \\
\hline 2 & 0.803 & 0.006 & 0.019 & 0.51 & 0.041 & 11 \\
\hline 3 & 0.765 & 0.011 & 0.010 & 0.52 & 0.113 & 8 \\
\hline 4 & 0.895 & -0.020 & 0.007 & 0.81 & 0.084 & 5 \\
\hline 5 & 0.945 & -0.023 & -0.021 & 0.90 & 0.034 & 5 \\
\hline 6 & 0.959 & -0.014 & -0.015 & 0.27 & 0.536 & 6 \\
\hline 7 & 1.016 & -0.009 & 0.015 & 0.33 & 0.366 & 7 \\
\hline 8 & 1.023 & 0.005 & 0.010 & 0.09 & 0.418 & 20 \\
\hline 9 & 1.083 & -0.000 & 0.003 & 0.02 & 0.676 & 48 \\
\hline 10 & 1.077 & 0.003 & 0.007 & 0.24 & 0.070 & 98 \\
\hline 11 & 1.069 & 0.003 & 0.007 & 0.28 & 0.000 & 139 \\
\hline 12 & 1.055 & 0.003 & 0.008 & 0.30 & 0.000 & 164 \\
\hline 13 & 1.047 & 0.003 & 0.008 & 0.31 & 0.000 & 169 \\
\hline 14 & 1.043 & 0.004 & 0.008 & 0.34 & 0.000 & 168 \\
\hline 15 & 1.037 & 0.004 & 0.007 & 0.31 & 0.000 & 170 \\
\hline 16 & 1.028 & 0.003 & 0.007 & 0.29 & 0.000 & 168 \\
\hline 17 & 1.047 & 0.003 & 0.007 & 0.21 & 0.000 & 167 \\
\hline 18 & 1.118 & 0.002 & 0.005 & 0.06 & 0.008 & 153 \\
\hline 19 & 1.145 & 0.003 & 0.007 & 0.12 & 0.001 & 119 \\
\hline 20 & 1.159 & 0.004 & 0.009 & 0.16 & 0.000 & 95 \\
\hline 21 & 1.158 & 0.003 & 0.006 & 0.16 & 0.003 & 69 \\
\hline 22 & 1.147 & 0.003 & 0.003 & 0.09 & 0.096 & 53 \\
\hline 23 & 1.094 & 0.003 & -0.000 & 0.11 & 0.142 & 35 \\
\hline 24 & 1.011 & 0.001 & -0.003 & 0.03 & 0.672 & 27 \\
\hline
\end{tabular}


LRD Regression Coefficients for Coastal Lodging - Non-Standard Day - Summer

\begin{tabular}{|c|c|c|c|c|c|c|}
\hline Hour & $\begin{array}{l}\text { Reg } \\
\text { Base Load } \\
\text { Intercept } \\
\left(W / f^{2}\right)\end{array}$ & $\begin{array}{c}\text { ssion Paran } \\
\text { Dew Point } \\
\text { Coefficeint } \\
\left(W / t^{2} /{ }^{\circ} F\right)\end{array}$ & $\begin{array}{l}\text { ters } \\
\text { Dry Bulb } \\
\text { Coefficient } \\
\left(\mathrm{W} / \mathrm{ft}^{2} / \circ \mathrm{F}\right)\end{array}$ & $\mathrm{R}^{2}$ & $\begin{array}{c}\text { Statistics } \\
\text { Significance } \\
\text { of } \\
\text { F-Statistic }\end{array}$ & $N$ \\
\hline 1 & 0.000 & 0.000 & 0.000 & 0.00 & 1.000 & 0 \\
\hline $\begin{array}{l}2 \\
3\end{array}$ & $\begin{array}{l}0.000 \\
0.000\end{array}$ & $\begin{array}{l}0.000 \\
0.000\end{array}$ & $\begin{array}{l}0.000 \\
0.000\end{array}$ & $\begin{array}{l}0.00 \\
0.00\end{array}$ & $\begin{array}{l}1.000 \\
1.000\end{array}$ & $\begin{array}{l}0 \\
0\end{array}$ \\
\hline 4 & 0.000 & 0.000 & 0.000 & 0.00 & 1.000 & 0 \\
\hline 5 & 0.000 & 0.000 & 0.000 & 0.00 & 1.000 & 0 \\
\hline 6 & 0.000 & 0.000 & 0.000 & 0.00 & 1.000 & 0 \\
\hline 7 & 0.000 & 0.000 & 0.000 & 0.00 & 1.000 & 0 \\
\hline 8 & 0.000 & 0.000 & 0.000 & 0.00 & 1.000 & 0 \\
\hline 9 & 0.000 & 0.000 & 0.000 & 0.00 & 1.000 & 0 \\
\hline 10 & 0.000 & 0.000 & 0.000 & 0.00 & 1.000 & 0 \\
\hline 11 & 0.000 & 0.000 & 0.000 & 0.00 & 1.000 & 0 \\
\hline 12 & 0.000 & 0.000 & 0.000 & 0.00 & 1.000 & 0 \\
\hline 13 & 0.000 & 0.000 & 0.000 & 0.00 & 1.000 & 0 \\
\hline 14 & 0.000 & 0.000 & 0.000 & 0.00 & 1.000 & 0 \\
\hline 15 & 0.000 & 0.000 & 0.000 & 0.00 & 1.000 & 0 \\
\hline 16 & 0.000 & 0.000 & 0.000 & 0.00 & 1.000 & 0 \\
\hline 17 & 0.000 & 0.000 & 0.000 & 0.00 & 1.000 & 0 \\
\hline 18 & 0.000 & 0.000 & 0.000 & 0.00 & 1.000 & 0 \\
\hline 19 & 0.000 & 0.000 & 0.000 & 0.00 & 1.000 & 0 \\
\hline 20 & 0.000 & 0.000 & 0.000 & 0.00 & 1.000 & 0 \\
\hline 21 & 0.000 & 0.000 & 0.000 & 0.00 & 1.000 & 0 \\
\hline 22 & 0.000 & 0.000 & 0.000 & 0.00 & 1.000 & 0 \\
\hline 23 & 0.000 & 0.000 & 0.000 & 0.00 & 1.000 & 0 \\
\hline 24 & 0.000 & 0.000 & 0.000 & 0.00 & 1.000 & 0 \\
\hline
\end{tabular}

LRD Regression Coefficients for Coastal Lodging - Non-Standard Day - Winter

\begin{tabular}{|c|c|c|c|c|c|c|}
\hline \multirow[b]{2}{*}{ Hour } & \multicolumn{3}{|c|}{ Regression Parameters } & \\
\hline & $\begin{array}{l}\text { Base Load } \\
\text { Intercept } \\
\left(\mathrm{W} / \mathrm{ft}^{2}\right)\end{array}$ & $\begin{array}{l}\text { Dew Point } \\
\text { Coefficeint } \\
\left(W / t^{2} /{ }^{\circ} \mathrm{F}\right)\end{array}$ & $\begin{array}{l}\text { Dry Bulb } \\
\text { Coefficient } \\
\left(\mathrm{W} / \mathrm{ft}^{2} /{ }^{\circ} \mathrm{F}\right)\end{array}$ & $\mathrm{R}^{2}$ & $\begin{array}{c}\text { Significance } \\
\text { of } \\
\text { F-Statistic }\end{array}$ & $N$ \\
\hline 1 & 0.000 & 0.000 & 0.000 & 0.00 & 1.000 & 0 \\
\hline 2 & 0.000 & 0.000 & 0.000 & 0.00 & 1.000 & 0 \\
\hline 3 & 0.000 & 0.000 & 0.000 & 0.00 & 1.000 & 0 \\
\hline 4 & 0.000 & 0.000 & 0.000 & 0.00 & 1.000 & 0 \\
\hline 5 & 0.000 & 0.000 & 0.000 & 0.00 & 1.000 & 0 \\
\hline 6 & 0.000 & 0.000 & 0.000 & 0.00 & 1.000 & 0 \\
\hline 7 & 0.000 & 0.000 & 0.000 & 0.00 & 1.000 & 0 \\
\hline 8 & 0.000 & 0.000 & 0.000 & 0.00 & 1.000 & 0 \\
\hline 9 & 0.000 & 0.000 & 0.000 & 0.00 & 1.000 & 0 \\
\hline 10 & 0.000 & 0.000 & 0.000 & 0.00 & 1.000 & 0 \\
\hline 11 & 0.000 & 0.000 & 0.000 & 0.00 & 1.000 & 0 \\
\hline 12 & 0.000 & 0.000 & 0.000 & 0.00 & 1.000 & 0 \\
\hline 13 & 0.000 & 0.000 & 0.000 & 0.00 & 1.000 & 0 \\
\hline 14 & 0.000 & 0.000 & 0.000 & 0.00 & 1.000 & 0 \\
\hline 15 & 0.000 & 0.000 & 0.000 & 0.00 & 1.000 & 0 \\
\hline 16 & 0.000 & 0.000 & 0.000 & 0.00 & 1.000 & 0 \\
\hline 17 & 0.000 & 0.000 & 0.000 & 0.00 & 1.000 & 0 \\
\hline 18 & 0.000 & 0.000 & 0.000 & 0.00 & 1.000 & 0 \\
\hline 19 & 0.000 & 0.000 & 0.000 & 0.00 & 1.000 & 0 \\
\hline 20 & 0.000 & 0.000 & 0.000 & 0.00 & 1.000 & 0 \\
\hline 21 & 0.000 & 0.000 & 0.000 & 0.00 & 1.000 & 0 \\
\hline 22 & 0.000 & 0.000 & 0.000 & 0.00 & 1.000 & 0 \\
\hline 23 & 0.000 & 0.000 & 0.000 & 0.00 & 1.000 & 0 \\
\hline 24 & 0.000 & 0.000 & 0.000 & 0.00 & 1.000 & 0 \\
\hline
\end{tabular}


LRD Regression Coefficients for Non-Coastal Lodging - Standard Day - Summer

\begin{tabular}{|c|c|c|c|c|c|c|}
\hline \multirow[b]{2}{*}{ Hour } & \multicolumn{3}{|c|}{ Regression Parameters } & \multicolumn{3}{|c|}{ Statistics } \\
\hline & $\begin{array}{l}\text { Base Load } \\
\text { Intercept } \\
\left(W / t^{2}\right)\end{array}$ & $\begin{array}{l}\text { Dew Point } \\
\text { Coefficeint } \\
\left(W / t^{2} /{ }^{\circ} F\right)\end{array}$ & $\begin{array}{l}\text { Dry Bulb } \\
\text { Coefficient } \\
\left(W / t^{2} /{ }^{\circ} F\right)\end{array}$ & $R^{2}$ & $\begin{array}{c}\text { Significance } \\
\text { of } \\
\text { F-Statistic }\end{array}$ & $N$ \\
\hline 1 & 1.179 & 0.006 & 0.014 & 0.45 & 0.000 & 150 \\
\hline 2 & 1.154 & 0.005 & 0.013 & 0.38 & 0.000 & 145 \\
\hline 3 & 1.133 & 0.005 & 0.013 & 0.49 & 0.000 & 139 \\
\hline 4 & 1.127 & 0.006 & 0.012 & 0.42 & 0.000 & 133 \\
\hline 5 & 1.136 & 0.006 & 0.011 & 0.38 & 0.000 & 125 \\
\hline 6 & 1.215 & 0.004 & 0.011 & 0.39 & 0.000 & 119 \\
\hline 7 & 1.316 & 0.003 & 0.008 & 0.22 & 0.000 & 126 \\
\hline 8 & 1.343 & 0.007 & 0.007 & 0.34 & 0.000 & 139 \\
\hline 9 & 1.383 & 0.008 & 0.009 & 0.58 & 0.000 & 171 \\
\hline 10 & 1.338 & 0.011 & 0.011 & 0.47 & 0.000 & 180 \\
\hline 11 & 1.321 & 0.011 & 0.012 & 0.62 & 0.000 & 180 \\
\hline 12 & 1.321 & 0.010 & 0.012 & 0.75 & 0.000 & 181 \\
\hline 13 & 1.329 & 0.011 & 0.013 & 0.77 & 0.000 & 180 \\
\hline 14 & 1.318 & 0.011 & 0.014 & 0.75 & 0.000 & 180 \\
\hline 15 & 1.272 & 0.012 & 0.015 & 0.76 & 0.000 & 181 \\
\hline 16 & 1.249 & 0.010 & 0.016 & 0.73 & 0.000 & 178 \\
\hline 17 & 1.321 & 0.010 & 0.016 & 0.74 & 0.000 & 181 \\
\hline 18 & 1.375 & 0.013 & 0.016 & 0.76 & 0.000 & 182 \\
\hline 19 & 1.417 & 0.014 & 0.016 & 0.77 & 0.000 & 180 \\
\hline 20 & 1.410 & 0.009 & 0.015 & 0.66 & 0.000 & 179 \\
\hline 21 & 1.385 & 0.009 & 0.015 & 0.62 & 0.000 & 178 \\
\hline 22 & 1.297 & 0.010 & 0.016 & 0.51 & 0.000 & 174 \\
\hline 23 & 1.248 & 0.008 & 0.015 & 0.49 & 0.000 & 163 \\
\hline 24 & 1.219 & 0.005 & 0.014 & 0.44 & 0.000 & 155 \\
\hline
\end{tabular}

LRD Regression Coefficients for Non-Coastal Lodging - Standard Day - Winter

\begin{tabular}{|c|c|c|c|c|c|c|}
\hline \multirow[b]{2}{*}{ Hour } & \multicolumn{3}{|c|}{ Regression Parameters } & \multicolumn{3}{|c|}{ Statistics } \\
\hline & $\begin{array}{l}\text { Base Load } \\
\text { Intercept } \\
\left(W / t^{2}\right)\end{array}$ & $\begin{array}{l}\text { Dew Point } \\
\text { Coefficeint } \\
\left(\mathrm{W} / \mathrm{tt}^{2} /{ }^{\circ} \mathrm{F}\right)\end{array}$ & $\begin{array}{l}\text { Dry Bulb } \\
\text { Coefficient } \\
\left(\mathrm{W} / \mathrm{t}^{2} /{ }^{\circ} \mathrm{F}\right)\end{array}$ & $R^{2}$ & $\begin{array}{c}\text { Significance } \\
\text { of } \\
\text { F-Statistic }\end{array}$ & $N$ \\
\hline 1 & 1.221 & 0.002 & 0.000 & 0.05 & 0.492 & 28 \\
\hline 2 & 1.164 & 0.001 & 0.001 & 0.01 & 0.898 & 20 \\
\hline 3 & 1.145 & 0.001 & -0.003 & 0.04 & 0.763 & 17 \\
\hline 4 & 1.126 & -0.001 & -0.004 & 0.08 & 0.602 & 14 \\
\hline 5 & 1.137 & -0.000 & -0.004 & 0.04 & 0.776 & 14 \\
\hline 6 & 1.215 & 0.001 & 0.005 & 0.08 & 0.527 & 17 \\
\hline 7 & 1.332 & 0.002 & 0.001 & 0.39 & 0.065 & 13 \\
\hline 8 & 1.355 & 0.004 & 0.005 & 0.28 & 0.085 & 17 \\
\hline 9 & 1.459 & 0.005 & 0.002 & 0.30 & 0.000 & 51 \\
\hline 10 & 1.443 & 0.006 & 0.007 & 0.31 & 0.000 & 91 \\
\hline 11 & 1.411 & 0.006 & 0.008 & 0.31 & 0.000 & 130 \\
\hline 12 & 1.386 & 0.006 & 0.009 & 0.38 & 0.000 & 155 \\
\hline 13 & 1.398 & 0.006 & 0.009 & 0.39 & 0.000 & 160 \\
\hline 14 & 1.397 & 0.006 & 0.008 & 0.37 & 0.000 & 165 \\
\hline 15 & 1.353 & 0.005 & 0.008 & 0.36 & 0.000 & 163 \\
\hline 16 & 1.343 & 0.006 & 0.009 & 0.36 & 0.000 & 161 \\
\hline 17 & 1.428 & 0.004 & 0.006 & 0.26 & 0.000 & 161 \\
\hline 18 & 1.533 & 0.002 & 0.004 & 0.12 & 0.000 & 158 \\
\hline 19 & 1.555 & 0.003 & 0.007 & 0.18 & 0.000 & 133 \\
\hline 20 & 1.463 & 0.004 & 0.011 & 0.29 & 0.000 & 109 \\
\hline 21 & 1.409 & 0.004 & 0.012 & 0.30 & 0.000 & 84 \\
\hline 22 & 1.342 & 0.004 & 0.011 & 0.25 & 0.000 & 65 \\
\hline 23 & 1.298 & 0.003 & 0.007 & 0.14 & 0.015 & 56 \\
\hline 24 & 1.236 & 0.003 & 0.008 & 0.14 & 0.047 & 14 \\
\hline
\end{tabular}


LRD Regression Coefficlents for Non-Coastal Lodging - Non-Standard Day - Summer

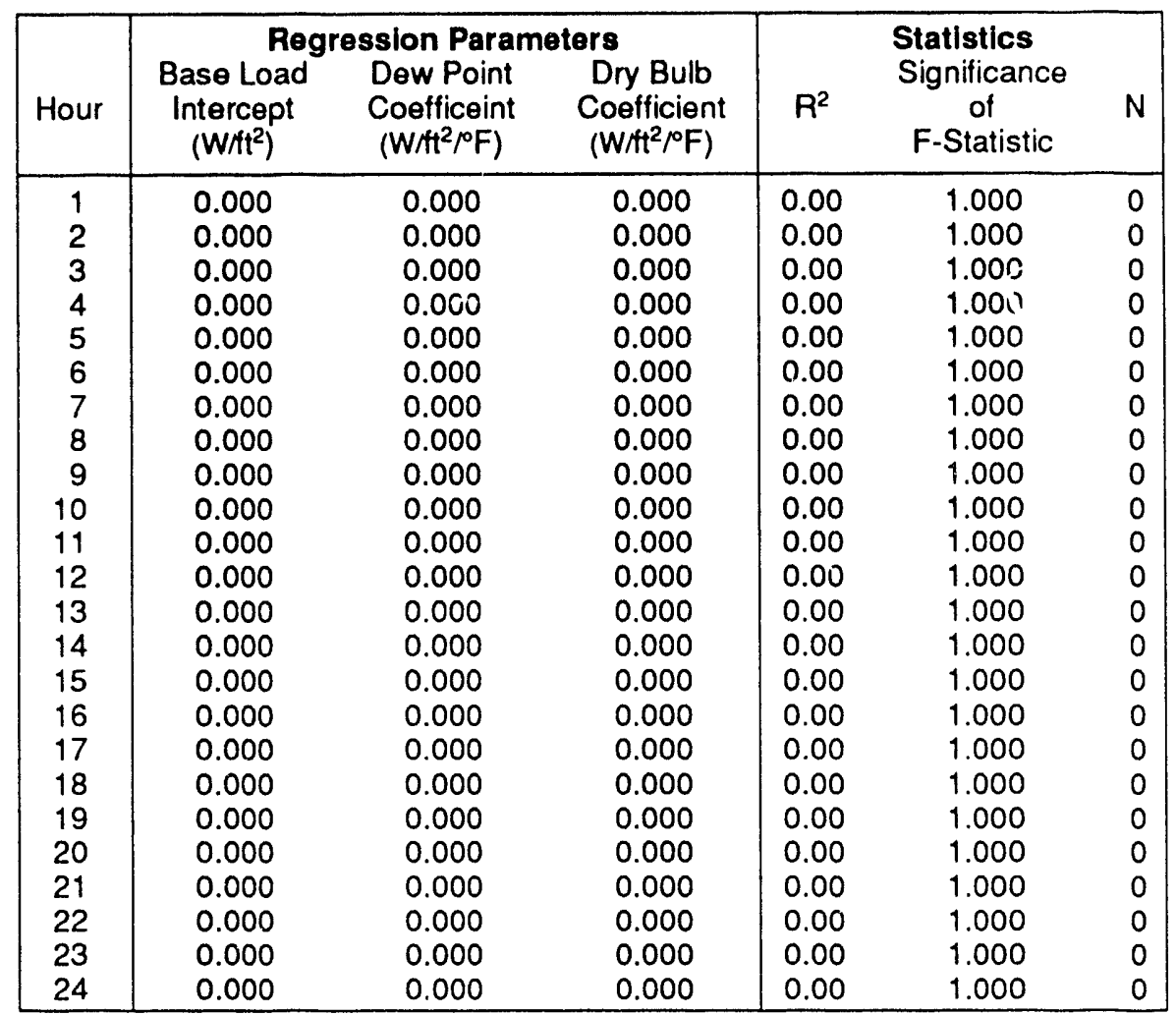

LRD Regression Coefficients for Non-Coastal Lodging - Non-Standard D yy - Winter

\begin{tabular}{|c|c|c|c|c|c|c|}
\hline \multirow[b]{2}{*}{ Hour } & \multicolumn{3}{|c|}{ Regression Parameters } & \multicolumn{3}{|c|}{$\begin{array}{l}\text { Statistics } \\
\text { Sianificance }\end{array}$} \\
\hline & $\begin{array}{l}\text { Intercept } \\
\left(W / t^{2}\right)\end{array}$ & $\begin{array}{l}\text { Dew Point } \\
\text { Coefficeint } \\
\left(\mathrm{W} / \mathrm{t}^{2} /{ }^{\circ} \mathrm{F}\right)\end{array}$ & $\begin{array}{l}\text { Coefficient } \\
\left.\text { (W/ft } /{ }^{\circ} \mathrm{F}\right)\end{array}$ & $\mathrm{R}^{2}$ & $\begin{array}{c}\text { Significance } \\
\text { of } \\
\text { F-Statistic }\end{array}$ & $N$ \\
\hline 1 & 0.000 & 0.000 & 0.000 & 0.00 & 1.000 & 0 \\
\hline 2 & 0.000 & 0.000 & 0.000 & 0.00 & 1.000 & 0 \\
\hline 3 & 0.000 & 0.000 & 0.000 & 0.00 & 1.000 & 0 \\
\hline 4 & 0.000 & 0.000 & 0.000 & 0.00 & 1.000 & 0 \\
\hline 5 & 0.000 & 0.000 & 0.000 & 0.00 & 1.000 & 0 \\
\hline 6 & 0.000 & 0.000 & 0.000 & 0.00 & 1.000 & 0 \\
\hline 7 & 0.000 & 0.000 & 0.000 & 0.00 & 1.000 & 0 \\
\hline 8 & 0.000 & 0.000 & 0.000 & 0.00 & 1.000 & 0 \\
\hline 9 & 0.000 & 0.000 & 0.000 & 0.00 & 1.000 & 0 \\
\hline 10 & 0.000 & 0.000 & 0.000 & 0.00 & 1.000 & 0 \\
\hline 11 & 0.000 & 0.000 & 0.000 & 0.00 & 1.000 & 0 \\
\hline 12 & 0.000 & 0.000 & 0.000 & 0.00 & 1.000 & 0 \\
\hline 13 & 0.000 & 0.000 & 0.000 & 0.00 & 1.000 & 0 \\
\hline 14 & 0.000 & 0.000 & 0.000 & 0.00 & 1.000 & 0 \\
\hline 15 & 0.000 & 0.000 & 0.000 & 0.00 & 1.000 & 0 \\
\hline 16 & 0.000 & 0.000 & 0.000 & 0.00 & 1.000 & 0 \\
\hline 17 & 0.000 & 0.000 & 0.000 & 0.00 & 1.000 & 0 \\
\hline 18 & 0.000 & 0.000 & 0.000 & 0.00 & 1.000 & 0 \\
\hline 19 & 0.000 & 0.000 & 0.000 & 0.00 & 1.000 & 0 \\
\hline 20 & 0.000 & 0.000 & 0.000 & 0.00 & 1.000 & 0 \\
\hline 21 & 0.000 & 0.000 & 0.000 & 0.00 & 1.000 & 0 \\
\hline 22 & 0.000 & 0.000 & 0.000 & 0.00 & 1.000 & 0 \\
\hline 23 & 0.000 & 0.000 & 0.000 & 0.00 & 1.000 & 0 \\
\hline 24 & 0.000 & 0.000 & 0.000 & 0.00 & 1.000 & 0 \\
\hline
\end{tabular}


LRD Regression Coefficients for Coastal School - Standard Day - Summer

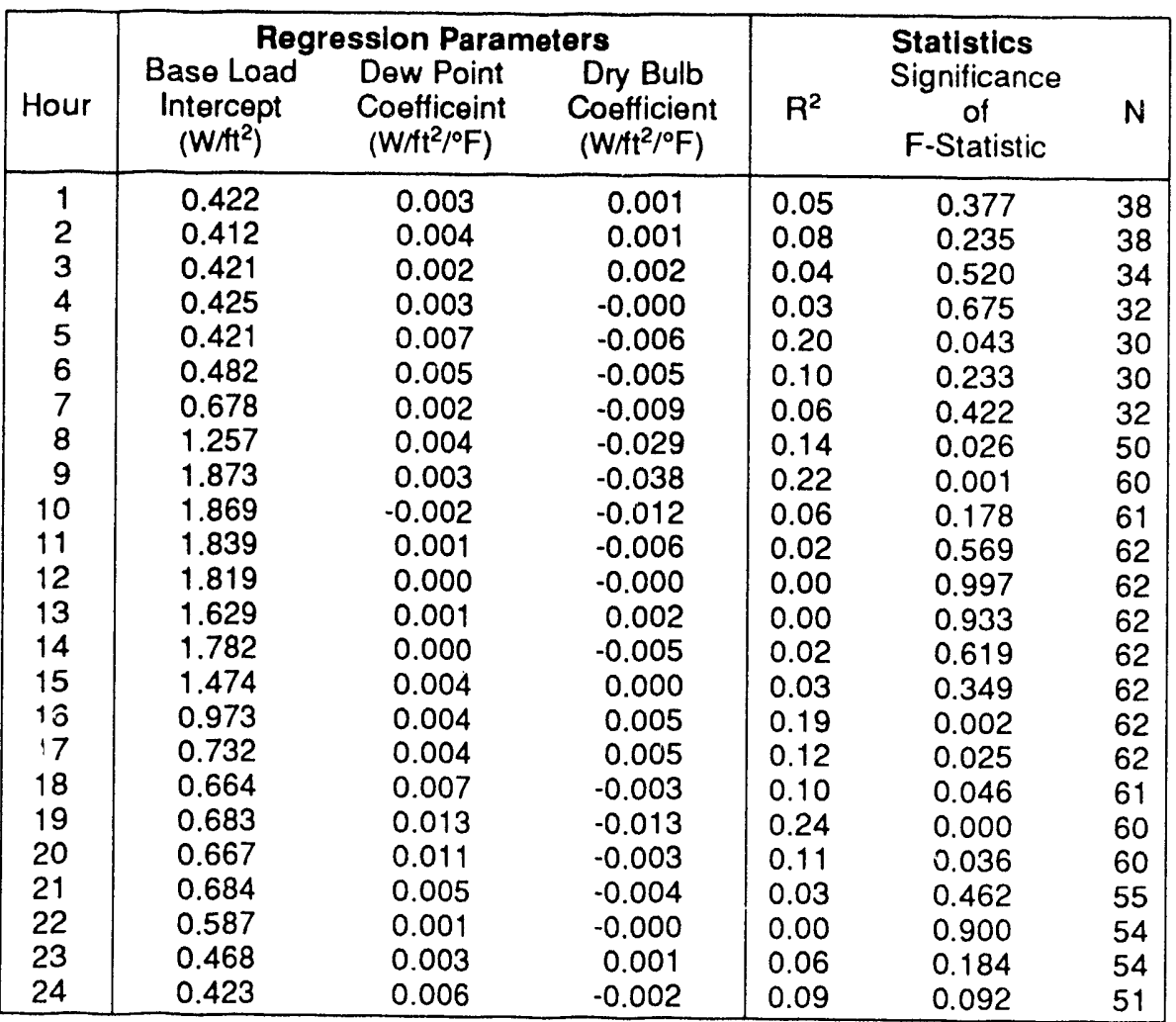

LRD Regression Coefficients for Coastal School - Standard Day - Winter

\begin{tabular}{|c|c|c|c|c|c|c|}
\hline \multirow[b]{2}{*}{ Hour } & \multicolumn{3}{|c|}{ Regression Parameters } & \multicolumn{3}{|c|}{$\begin{array}{l}\text { Statistics } \\
\text { Significance }\end{array}$} \\
\hline & $\begin{array}{l}\text { Base Load } \\
\text { Intercept } \\
\left(\mathrm{W} / \mathrm{ft}^{2}\right)\end{array}$ & $\begin{array}{l}\text { Dew Point } \\
\text { Coefficeint } \\
\left(\mathrm{W} / \mathrm{ft}^{2} /{ }^{\circ} \mathrm{F}\right)\end{array}$ & $\begin{array}{l}\text { Dry Bulb } \\
\text { Coefficient } \\
\left(\mathrm{W} / \mathrm{t}^{2} /{ }^{\circ} \mathrm{F}\right)\end{array}$ & $R^{2}$ & $\begin{array}{l}\text { Significance } \\
\text { of } \\
\text { F-Statistic }\end{array}$ & $\mathrm{N}$ \\
\hline 1 & 0.339 & 0.012 & 0.028 & 0.54 & 0.009 & 14 \\
\hline 2 & 0.352 & 0.010 & 0.047 & 0.85 & 0.001 & 9 \\
\hline 3 & 0.419 & 0.004 & 0.038 & 0.66 & 0.068 & 7 \\
\hline 4 & 0.170 & 0.061 & 0.064 & 0.99 & 0.005 & 4 \\
\hline 5 & 0.399 & 0.014 & 0.055 & 0.84 & 0.159 & 4 \\
\hline 6 & 0.515 & -0.002 & 0.020 & 0.42 & 0.444 & 5 \\
\hline 7 & 0.648 & -0.006 & 0.019 & 0.40 & 0.361 & 6 \\
\hline 8 & 1.057 & 0.011 & 0.011 & 0.02 & 0.851 & 15 \\
\hline 9 & 1.620 & -0.003 & -0.015 & 0.01 & 0.789 & 37 \\
\hline 10 & 1.619 & 0.000 & -0.001 & 0.00 & 0.996 & 65 \\
\hline 11 & 1.669 & 0.004 & -0.005 & 0.01 & 0.521 & 96 \\
\hline 12 & 1.541 & 0.005 & 0.011 & 0.01 & 0.437 & 112 \\
\hline 13 & 1.381 & 0.005 & 0.010 & 0.02 & 0.325 & 116 \\
\hline 14 & 1.437 & 0.007 & 0.012 & 0.03 & 0.210 & 115 \\
\hline 15 & 1.275 & 0.003 & 0.008 & 0.01 & 0.430 & 115 \\
\hline 16 & 0.903 & 0.001 & 0.006 & 0.03 & 0.227 & 116 \\
\hline 17 & 0.715 & 0.000 & 0.003 & 0.01 & 0.559 & 117 \\
\hline 18 & 0.715 & 0.000 & -0.002 & 0.01 & 0.733 & 105 \\
\hline 19 & 0.720 & 0.000 & -0.001 & 0.00 & 0.971 & 79 \\
\hline 20 & 0.774 & -0.001 & -0.006 & 0.02 & 0.574 & 63 \\
\hline 21 & 0.715 & 0.001 & 0.001 & 0.01 & 0.835 & 43 \\
\hline 22 & 0.622 & -0.000 & 0.001 & 0.00 & 0.993 & 29 \\
\hline 23 & 0.499 & 0.001 & 0.003 & 0.02 & 0.787 & 21 \\
\hline 24 & 0.441 & 0.002 & -0.003 & 0.08 & 0.559 & 16 \\
\hline
\end{tabular}


LRD Regression Coefficients for Coastal School - Non-Standard Day - Summer

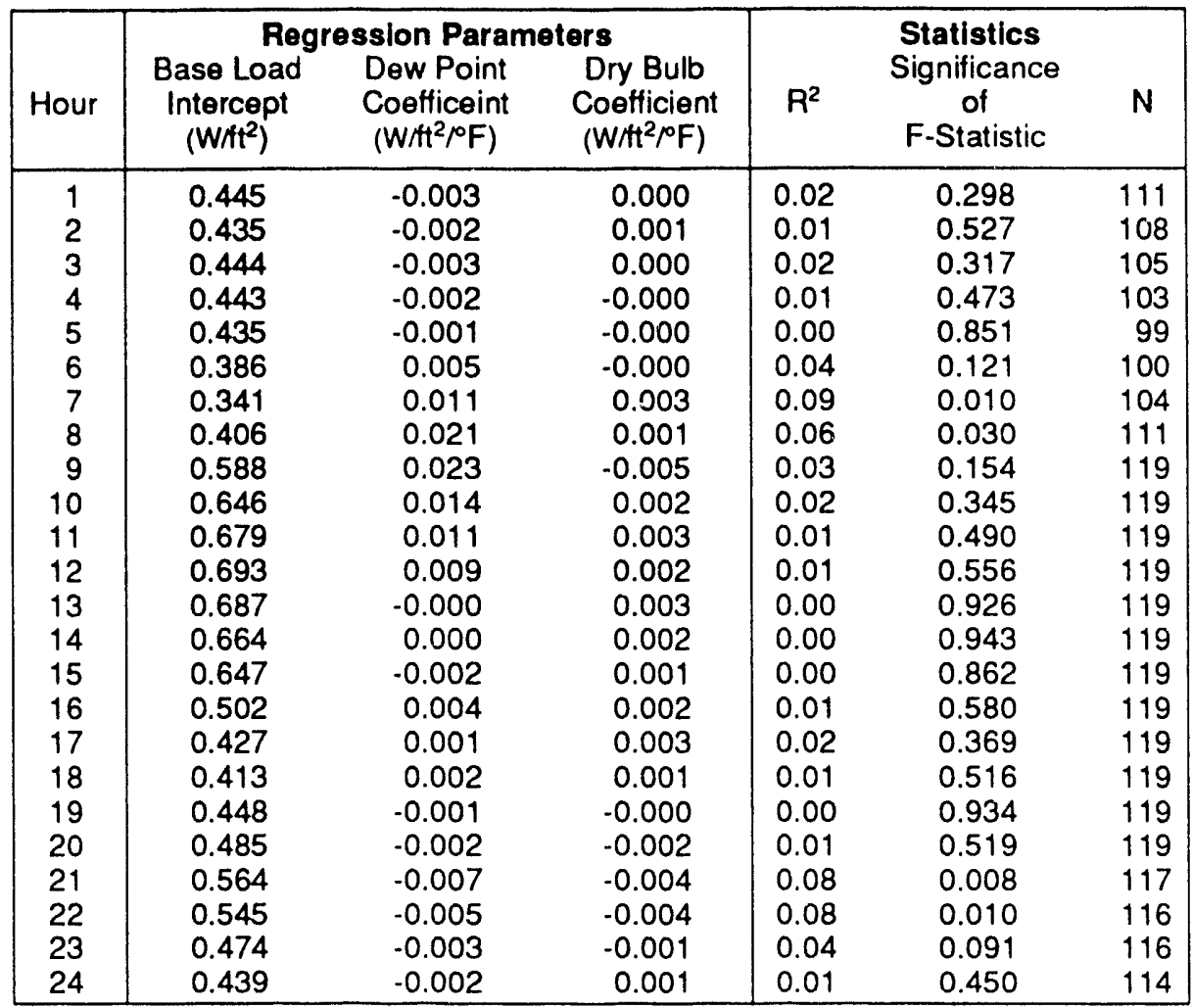

LRD Regression Coefficients for Coastal School - Non-Standard Day - Winter

\begin{tabular}{|c|c|c|c|c|c|c|}
\hline \multirow[b]{2}{*}{ Hour } & \multicolumn{3}{|c|}{ Regression Parameters } & \\
\hline & $\begin{array}{l}\text { Base Load } \\
\text { Intercept } \\
\left(\mathrm{W} / \mathrm{tt}^{2}\right)\end{array}$ & $\begin{array}{l}\text { Dew Point } \\
\text { Coefficeint } \\
\left(\mathrm{W} / \mathrm{t}^{2} /{ }^{\circ} \mathrm{F}\right)\end{array}$ & $\begin{array}{l}\text { Dry Bulb } \\
\text { Coefficient } \\
\left(\text { W } / \mathrm{ft}^{2} /{ }^{\circ} \mathrm{F}\right)\end{array}$ & $\mathrm{R}^{2}$ & $\begin{array}{c}\text { Significance } \\
\text { of } \\
\text { F-Statistic }\end{array}$ & $N$ \\
\hline 1 & 0.441 & -0.004 & -0.023 & 0.10 & 0.850 & 5 \\
\hline 2 & 85.191 & 0.689 & 0.846 & 1.00 & 2.000 & 0 \\
\hline 3 & 0.000 & 0.000 & 0.000 & 0.00 & 1.000 & 0 \\
\hline 4 & 0.000 & 0.000 & 0.000 & 0.00 & 1.000 & 0 \\
\hline 5 & 0.000 & 0.000 & 0.000 & 0.00 & 1.000 & 0 \\
\hline 6 & 0.000 & 0.000 & 0.000 & 0.00 & 1.000 & 0 \\
\hline 7 & 0.000 & 0.000 & 0.000 & 0.00 & 1.000 & 0 \\
\hline 8 & 0.356 & -0.004 & 0.018 & 0.81 & 0.190 & 4 \\
\hline 9 & 0.702 & -0.034 & -0.065 & 0.61 & 0.024 & 10 \\
\hline 10 & 0.398 & -0.008 & 0.010 & 0.14 & 0.098 & 32 \\
\hline 11 & 0.426 & -0.008 & 0.001 & 0.12 & 0.079 & 42 \\
\hline 12 & 0.398 & -0.005 & 0.004 & 0.12 & 0.046 & 51 \\
\hline 13 & 0.393 & -0.004 & 0.003 & 0.12 & 0.044 & 52 \\
\hline 14 & 0.384 & -0.001 & 0.005 & 0.07 & 0.186 & 52 \\
\hline 15 & 0.383 & -0.001 & 0.005 & 0.07 & 0.138 & 54 \\
\hline 16 & 0.388 & -0.000 & 0.004 & 0.07 & 0.157 & 51 \\
\hline 17 & 0.446 & -0.003 & -0.002 & 0.06 & 0.215 & 49 \\
\hline 18 & 0.492 & -0.005 & -0.004 & 0.10 & 0.085 & 47 \\
\hline 19 & 0.500 & -0.003 & -0.001 & 0.05 & 0.365 & 39 \\
\hline 20 & 0.516 & -0.002 & 0.001 & 0.02 & 0.739 & 31 \\
\hline 21 & 0.518 & -0.001 & 0.002 & 0.01 & 0.897 & 25 \\
\hline 22 & 0.494 & -0.001 & 0.003 & 0.01 & 0.886 & 23 \\
\hline 23 & 0.389 & 0.004 & 0.014 & 0.16 & 0.396 & 13 \\
\hline 24 & 0.404 & -0.005 & 0.007 & 0.30 & 0.245 & 10 \\
\hline
\end{tabular}


LRD Regression Coefficients for Non-Coastal School - Standard Day - Summer

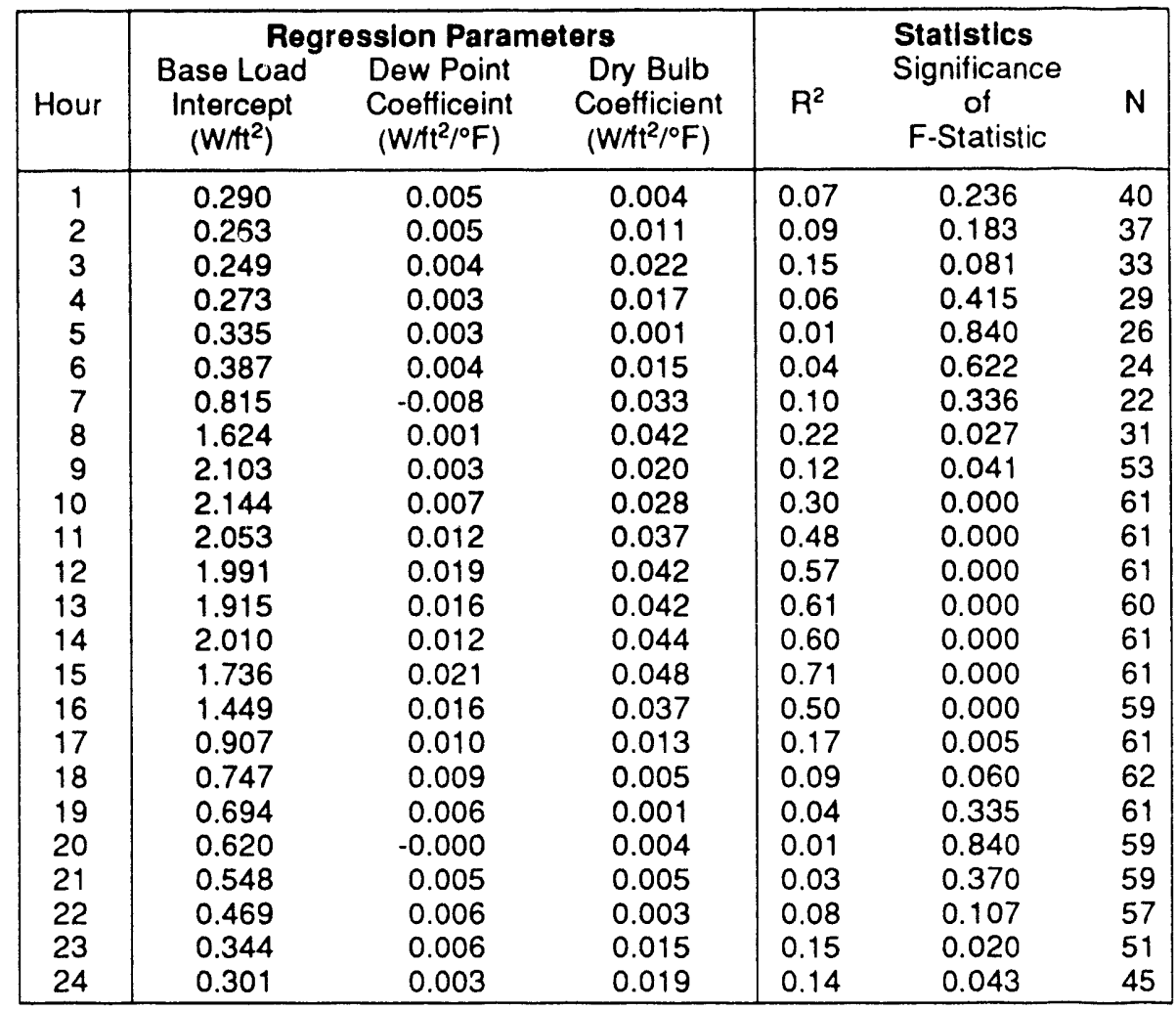

LRD Regression Coefficients for Non-Coastal School - Standard Day - Winter

\begin{tabular}{|c|c|c|c|c|c|c|}
\hline \multirow[b]{2}{*}{ Hour } & \multicolumn{3}{|c|}{ Regressior Parameters } & \multicolumn{3}{|c|}{ Statistics } \\
\hline & $\begin{array}{l}\text { Base Load } \\
\text { Intercept } \\
\left(\mathrm{W} / \mathrm{ft}^{2}\right)\end{array}$ & $\begin{array}{l}\text { Den Point } \\
\text { Coeticeint } \\
\left(\mathrm{W} / \mathrm{tt}^{2} / \mathrm{F}\right)\end{array}$ & $\begin{array}{l}\text { Dry Bulb } \\
\text { Coefficient } \\
\left(\mathrm{W} / \mathrm{t}^{2} /{ }^{\circ} \mathrm{F}\right)\end{array}$ & $\mathrm{R}^{2}$ & $\begin{array}{c}\text { Significance } \\
\text { of } \\
\text { F-Statistic }\end{array}$ & $\mathrm{N}$ \\
\hline 1 & 0.256 & 0.000 & -0.003 & 0.02 & 0.851 & 17 \\
\hline 2 & 0.270 & -0.001 & -0.008 & 0.11 & 0.438 & 16 \\
\hline 3 & 0.252 & -0.001 & -0.005 & 0.20 & 0.295 & 13 \\
\hline 4 & 0.234 & 0.002 & 0.001 & 0.14 & 0.539 & 10 \\
\hline 5 & 0.228 & 0.002 & 0.006 & 0.29 & 0.260 & 10 \\
\hline 6 & 0.310 & -0.005 & 0.012 & 0.26 & 0.229 & 12 \\
\hline 7 & 0.707 & -0.012 & 0.013 & 0.49 & 0.097 & 9 \\
\hline 8 & 1.501 & -0.008 & 0.024 & 0.49 & 0.069 & 10 \\
\hline 9 & 1.770 & -0.009 & 0.027 & 0.08 & 0.256 & 36 \\
\hline 10 & 2.005 & -0.003 & -0.003 & 0.00 & 0.866 & 62 \\
\hline 11 & 2.006 & 0.003 & 0.006 & 0.01 & 0.652 & 93 \\
\hline 12 & 1.873 & 0.012 & 0.025 & 0.11 & 0.001 & 111 \\
\hline 13 & 1.719 & 0.012 & 0.028 & 0.15 & 0.000 & 112 \\
\hline 14 & 1.836 & 0.014 & 0.028 & 0.14 & 0.000 & 116 \\
\hline 15 & 1.598 & 0.012 & 0.031 & 0.20 & 0.000 & 113 \\
\hline 16 & 1.264 & 0.009 & 0.023 & 0.17 & 0.000 & 112 \\
\hline 17 & 0.727 & 0.002 & 0.008 & 0.09 & 0.007 & 111 \\
\hline 18 & 0.632 & 0.000 & 0.002 & 0.01 & 0.734 & 110 \\
\hline 19 & 0.615 & 0.000 & -0.002 & 0.00 & 0.855 & 91 \\
\hline 20 & 0.567 & -0.001 & 0.000 & 0.00 & 0.942 & 77 \\
\hline 21 & 0.496 & -0.001 & 0.002 & 0.01 & 0.678 & 57 \\
\hline 22 & 0.406 & -0.001 & 0.004 & 0.02 & 0.629 & 42 \\
\hline 23 & 0.315 & -0.001 & 0.004 & 0.03 & 0.567 & 34 \\
\hline 24 & 0.267 & -0.001 & 0.003 & 0.03 & 0.706 & 26 \\
\hline
\end{tabular}


LRD Regression Coefficients for Non-Coastal School - Non-Standard Day - Summer

\begin{tabular}{|c|c|c|c|c|c|c|}
\hline Hour & $\begin{array}{l}\text { Reg } \\
\text { Base Load } \\
\text { Intercept } \\
\left(W / t^{2}\right)\end{array}$ & $\begin{array}{l}\text { ssion Parar } \\
\text { Dew Point } \\
\text { Coefficeint } \\
\left(W / t^{2} / F\right)\end{array}$ & $\begin{array}{l}\text { Dry Bulb } \\
\text { Coefficient } \\
\left(\mathrm{W} / \mathrm{t}^{2} / \mathrm{F}\right)\end{array}$ & $\mathrm{B}^{2}$ & $\begin{array}{c}\text { Statistics } \\
\text { Significance } \\
\text { of } \\
\text { F-Statistic }\end{array}$ & $N$ \\
\hline $\begin{array}{r}1 \\
2 \\
3 \\
4 \\
5 \\
6 \\
7 \\
8 \\
9 \\
10 \\
11 \\
12 \\
13 \\
14 \\
15 \\
16 \\
17 \\
18 \\
19 \\
20 \\
21 \\
22 \\
23 \\
24\end{array}$ & $\begin{array}{l}0.250 \\
0.240 \\
0.246 \\
0.249 \\
0.250 \\
0.252 \\
0.288 \\
0.404 \\
0.561 \\
0.549 \\
0.530 \\
0.565 \\
0.668 \\
0.655 \\
0.503 \\
0.352 \\
0.282 \\
0.322 \\
0.341 \\
0.333 \\
0.334 \\
0.336 \\
0.299 \\
0.262\end{array}$ & $\begin{array}{l}0.003 \\
0.002 \\
0.002 \\
0.002 \\
0.002 \\
0.004 \\
0.017 \\
0.040 \\
0.037 \\
0.036 \\
0.035 \\
0.036 \\
0.044 \\
0.048 \\
0.040 \\
0.030 \\
0.012 \\
0.005 \\
0.003 \\
0.004 \\
0.004 \\
0.003 \\
0.003 \\
0.002\end{array}$ & $\begin{array}{l}0.006 \\
0.007 \\
0.006 \\
0.005 \\
0.007 \\
0.015 \\
0.021 \\
0.017 \\
0.013 \\
0.017 \\
0.017 \\
0.014 \\
0.007 \\
0.007 \\
0.014 \\
0.017 \\
0.010 \\
0.004 \\
0.002 \\
0.002 \\
0.002 \\
0.000 \\
0.002 \\
0.005\end{array}$ & $\begin{array}{l}0.07 \\
0.07 \\
0.06 \\
0.05 \\
0.09 \\
0.22 \\
0.22 \\
0.12 \\
0.08 \\
0.10 \\
0.10 \\
0.09 \\
0.11 \\
0.10 \\
0.09 \\
0.10 \\
0.09 \\
0.04 \\
0.01 \\
0.03 \\
0.03 \\
0.02 \\
0.02 \\
0.06\end{array}$ & $\begin{array}{l}0.021 \\
0.023 \\
0.039 \\
0.063 \\
0.009 \\
0.000 \\
0.000 \\
0.002 \\
0.007 \\
0.003 \\
0.002 \\
0.004 \\
0.001 \\
0.002 \\
0.004 \\
0.003 \\
0.003 \\
0.091 \\
0.416 \\
0.205 \\
0.225 \\
0.390 \\
0.358 \\
0.039\end{array}$ & $\begin{array}{r}109 \\
107 \\
105 \\
103 \\
98 \\
94 \\
103 \\
107 \\
117 \\
118 \\
118 \\
119 \\
119 \\
118 \\
119 \\
118 \\
119 \\
119 \\
118 \\
119 \\
118 \\
116 \\
111 \\
109\end{array}$ \\
\hline
\end{tabular}

LRD Regression Coefficients for Non-Coastal School - Non-Standard Day - Winter

\begin{tabular}{|c|c|c|c|c|c|c|}
\hline \multirow[b]{2}{*}{ Hour } & \multicolumn{3}{|c|}{ Regression Parameters } & \\
\hline & $\begin{array}{l}\text { Base Load } \\
\text { Intercept } \\
\left(W / t^{2}\right)\end{array}$ & $\begin{array}{l}\text { Dew Point } \\
\text { Coefficeint } \\
\left(\mathrm{W} / \mathrm{ft}^{2} /{ }^{\circ} \mathrm{F}\right)\end{array}$ & $\begin{array}{l}\text { Dry Bulb } \\
\text { Coefficient } \\
\left(\mathrm{Wft}^{2} /{ }^{\circ} \mathrm{F}\right)\end{array}$ & $\mathrm{R}^{2}$ & $\begin{array}{c}\text { Significance } \\
\text { of } \\
\text { F-Statistic }\end{array}$ & $\mathrm{N}$ \\
\hline 1 & 0.225 & -0.000 & 0.002 & 0.01 & 0.942 & 10 \\
\hline 2 & 0.254 & 0.000 & -0.004 & 0.28 & 0.848 & 3 \\
\hline 3 & 0.001 & 0.000 & -0.004 & 0.00 & 0.941 & 4 \\
\hline 4 & 0.026 & 0.002 & -0.004 & 0.11 & 0.672 & 4 \\
\hline 5 & 0.043 & 0.002 & -0.004 & 0.25 & 0.499 & 4 \\
\hline 6 & 0.307 & 0.003 & 0.055 & 0.90 & 0.096 & 4 \\
\hline 7 & 0.503 & 0.012 & 0.019 & 1.00 & 0.070 & 3 \\
\hline 8 & 0.192 & -0.001 & 0.005 & 0.32 & 0.458 & 6 \\
\hline 9 & 0.338 & -0.041 & -0.084 & 0.46 & 0.024 & 14 \\
\hline 10 & 0.220 & -0.009 & 0.003 & 0.06 & 0.431 & 28 \\
\hline 11 & 0.237 & -0.010 & -0.000 & 0.08 & 0.265 & 36 \\
\hline 12 & 0.254 & -0.009 & -0.001 & 0.06 & 0.297 & 43 \\
\hline 13 & 0.251 & -0.006 & 0.001 & 0.04 & 0.395 & 47 \\
\hline 14 & 0.229 & -0.005 & 0.003 & 0.04 & 0.383 & 4,8 \\
\hline 15 & 0.211 & -0.003 & 0.006 & 0.04 & 0.355 & 49 \\
\hline 16 & 0.236 & -0.004 & 0.002 & 0.05 & 0.322 & 48 \\
\hline 17 & 0.252 & -0.003 & -0.000 & 0.05 & 0.297 & 49 \\
\hline 18 & 0.276 & -0.003 & -0.002 & 0.09 & 0.134 & 47 \\
\hline 19 & 0.275 & -0.003 & -0.003 & 0.12 & 0.081 & 41 \\
\hline 20 & 0.261 & -0.003 & -0.004 & 0.23 & 0.023 & 31 \\
\hline 21 & 0.262 & -0.003 & -0.006 & 0.27 & 0.024 & 26 \\
\hline 22 & 0.265 & -0.002 & -0.008 & 0.32 & 0.020 & 22 \\
\hline 23 & 0.244 & -0.001 & -0.005 & 0.22 & 0.099 & 21 \\
\hline 24 & 0.235 & -0.001 & -0.004 & 0.15 & 0.307 & 17 \\
\hline
\end{tabular}


LRD Regression Coefficients for Coastal College - Standard Day - Summer

\begin{tabular}{|c|c|c|c|c|c|c|}
\hline Hour & $\begin{array}{l}\text { Regr } \\
\text { Base Load } \\
\text { Intercept } \\
\left(W / t^{2}\right)\end{array}$ & $\begin{array}{l}\text { sslon Paran } \\
\text { Dew Point } \\
\text { Coefficeint } \\
\left(\mathrm{W} / \mathrm{ft}^{2} /{ }^{\circ} \mathrm{F}\right)\end{array}$ & $\begin{array}{l}\text { Dters } \\
\text { Dry Bulb } \\
\text { Coefficient } \\
\left(W / t^{2} /{ }^{\circ} F\right)\end{array}$ & $R^{2}$ & $\begin{array}{c}\text { Statistics } \\
\text { Significance } \\
\text { of } \\
\text { F-Statistic }\end{array}$ & $N$ \\
\hline $\begin{array}{r}1 \\
2 \\
3 \\
4 \\
5 \\
6 \\
7 \\
8 \\
9 \\
10 \\
11 \\
12 \\
13 \\
14 \\
15 \\
16 \\
17 \\
18 \\
19 \\
20 \\
21 \\
22 \\
23 \\
24\end{array}$ & $\begin{array}{l}0.774 \\
0.757 \\
0.758 \\
0.782 \\
0.818 \\
0.942 \\
1.212 \\
1.558 \\
1.732 \\
1.803 \\
1.811 \\
1.792 \\
1.800 \\
1.790 \\
1.771 \\
1.684 \\
1.538 \\
1.535 \\
1.523 \\
1.424 \\
1.208 \\
1.001 \\
0.865 \\
0.780\end{array}$ & $\begin{array}{r}-0.003 \\
-0.003 \\
-0.003 \\
-0.002 \\
0.001 \\
0.001 \\
-0.001 \\
-0.005 \\
-0.006 \\
-0.006 \\
-0.005 \\
-0.005 \\
-0.006 \\
-0.003 \\
-0.003 \\
-0.003 \\
-0.005 \\
-0.002 \\
0.001 \\
0.002 \\
0.004 \\
0.001 \\
0.003 \\
0.003\end{array}$ & $\begin{array}{r}0.001 \\
0.001 \\
0.001 \\
0.002 \\
0.006 \\
0.009 \\
0.013 \\
0.008 \\
0.002 \\
0.001 \\
0.003 \\
0.003 \\
0.003 \\
0.002 \\
0.000 \\
-0.001 \\
-0.002 \\
-0.008 \\
-0.013 \\
-0.009 \\
-0.003 \\
-0.014 \\
-0.064 \\
-0.003\end{array}$ & $\begin{array}{l}0.02 \\
0.02 \\
0.02 \\
0.03 \\
0.17 \\
0.27 \\
0.19 \\
0.06 \\
0.04 \\
0.04 \\
0.04 \\
0.05 \\
0.07 \\
0.02 \\
0.02 \\
0.02 \\
0.02 \\
0.03 \\
0.06 \\
0.03 \\
0.01 \\
0.01 \\
0.02 \\
0.03\end{array}$ & $\begin{array}{l}0.458 \\
0.376 \\
0.318 \\
0.299 \\
0.000 \\
0.000 \\
0.000 \\
0.037 \\
0.088 \\
0.076 \\
0.061 \\
0.030 \\
0.011 \\
0.229 \\
0.310 \\
0.391 \\
0.275 \\
0.117 \\
0.028 \\
0.195 \\
0.633 \\
0.486 \\
0.276 \\
0.218\end{array}$ & $\begin{array}{r}102 \\
102 \\
98 \\
96 \\
93 \\
93 \\
96 \\
114 \\
124 \\
125 \\
126 \\
126 \\
126 \\
126 \\
126 \\
126 \\
126 \\
125 \\
124 \\
124 \\
119 \\
118 \\
118 \\
115\end{array}$ \\
\hline
\end{tabular}

LRD Regression Coefficients for Coastal College - Standard Day - Winter

\begin{tabular}{|c|c|c|c|c|c|c|}
\hline \multirow[b]{2}{*}{ Hour } & \multicolumn{3}{|c|}{ Regression Parameters } & \multicolumn{3}{|c|}{ Statistics } \\
\hline & $\begin{array}{c}\text { Base Load } \\
\text { Intercept } \\
\left(W / t^{2}\right)\end{array}$ & $\begin{array}{l}\text { Dew Point } \\
\text { Cosfficeint } \\
\left.\forall \forall / f t^{2} /{ }^{\circ} F\right)\end{array}$ & $\begin{array}{l}\text { Dry Bulb } \\
\text { Coefficient } \\
\left(\text { W } / \mathrm{tt}^{2} /{ }^{\circ} \mathrm{F}\right)\end{array}$ & $R^{2}$ & $\begin{array}{c}\text { Significance } \\
\text { of } \\
\text { F-Statistic }\end{array}$ & $N$ \\
\hline 1 & 0.760 & 0.015 & -0.006 & 0.49 & 0.018 & 14 \\
\hline 2 & 0.740 & 0.013 & -0.007 & 0.40 & 0.164 & 9 \\
\hline 3 & 0.700 & 0.017 & -0.001 & 0.30 & 0.412 & 7 \\
\hline 4 & 1.366 & -0.133 & -0.049 & .00 & 0.004 & 4 \\
\hline 5 & 0.974 & -0.031 & -0.030 & 0.23 & 0.769 & 4 \\
\hline 6 & 0.815 & 0.012 & 0.050 & 0.12 & 0.830 & 5 \\
\hline 7 & 0.916 & 0.018 & 0.105 & 0.33 & 0.451 & 6 \\
\hline 8 & 1.311 & -0.001 & 0.071 & 0.35 & 0.061 & 15 \\
\hline 9 & 1.607 & -0.004 & 0.021 & 0.14 & 0.068 & 37 \\
\hline 10 & 1.677 & 0.005 & 0.017 & 0.12 & 0.017 & 65 \\
\hline 11 & 1.711 & 0.009 & 0.013 & 0.13 & 0.001 & 96 \\
\hline 12 & 1.627 & 0.011 & 0.021 & 0.17 & 0.000 & 112 \\
\hline 13 & 1.606 & 0.010 & 0.018 & 0.14 & 0.000 & 116 \\
\hline 14 & 1.611 & 0.011 & 0.018 & 0.14 & 0.000 & 115 \\
\hline 15 & 1.604 & 0.008 & 0.016 & 0.10 & 0.002 & 115 \\
\hline 16 & 1.558 & 0.005 & 0.015 & 0.09 & 0.004 & 116 \\
\hline 17 & 1.479 & 0.005 & 0.016 & 0.09 & 0.005 & 117 \\
\hline 18 & $1.4 \div 2$ & 0.005 & 0.014 & 0.05 & 0.072 & 105 \\
\hline 19 & 1.479 & 0.008 & 0.013 & 0.06 & 0.079 & 79 \\
\hline 20 & 1.471 & 0.005 & 0.011 & 0.03 & 0.448 & 63 \\
\hline 21 & 1.343 & 0.004 & 0.024 & 0.06 & 0.304 & 43 \\
\hline 22 & 1.203 & 0.004 & 0.017 & 0.04 & 0.554 & 29 \\
\hline 23 & 1.006 & 0.003 & 0.015 & 0.11 & 0.339 & 21 \\
\hline 24 & 0.868 & 0.002 & 0.030 & 0.20 & 0.207 & 16 \\
\hline
\end{tabular}


LRD Regression Coefficients for Coastal College - Non-Standard Day - Summer

\begin{tabular}{|c|c|c|c|c|c|c|}
\hline Hour & $\begin{array}{l}\text { Reg } \\
\text { Base Load } \\
\text { Intercept } \\
\left(W / t^{2}\right)\end{array}$ & $\begin{array}{l}\text { 8sion Parar } \\
\text { Dew Point } \\
\text { Coefficeint } \\
\left(\mathrm{W} / \mathrm{t}^{2} /{ }^{\circ} \mathrm{F}\right)\end{array}$ & $\begin{array}{l}\text { lers } \\
\text { Dry Bulb } \\
\text { Coefficient } \\
\left(\mathrm{W} f \mathrm{t}^{2} /{ }^{\circ} \mathrm{F}\right)\end{array}$ & $\mathrm{R}^{2}$ & $\begin{array}{l}\text { Statistics } \\
\text { Significance } \\
\text { of } \\
\text { F-Statistic }\end{array}$ & $N$ \\
\hline $\begin{array}{r}1 \\
2 \\
3 \\
4 \\
5 \\
6 \\
7 \\
8 \\
9 \\
10 \\
11 \\
12 \\
13 \\
14 \\
15 \\
16 \\
17 \\
18 \\
19 \\
20 \\
21 \\
22 \\
23 \\
24\end{array}$ & $\begin{array}{l}0.687 \\
0.674 \\
0.666 \\
0.665 \\
0.660 \\
0.634 \\
0.660 \\
0.803 \\
0.956 \\
0.993 \\
0.969 \\
0.991 \\
0.945 \\
0.971 \\
0.955 \\
0.903 \\
0.874 \\
0.892 \\
0.927 \\
0.907 \\
0.868 \\
0.828 \\
0.751 \\
0.673\end{array}$ & $\begin{array}{r}-0.000 \\
-0.002 \\
-0.001 \\
-0.001 \\
-0.002 \\
-0.001 \\
0.003 \\
-0.001 \\
-0.008 \\
-0.011 \\
-0.014 \\
-0.015 \\
-0.015 \\
-0.016 \\
-0.016 \\
-0.010 \\
-0.014 \\
-0.007 \\
-0.009 \\
-0.005 \\
-0.004 \\
-0.002 \\
-0.001 \\
0.002\end{array}$ & $\begin{array}{r}-0.002 \\
0.001 \\
0.001 \\
0.000 \\
0.002 \\
0.004 \\
0.001 \\
-0.005 \\
-0.008 \\
-0.002 \\
0.004 \\
0.003 \\
0.007 \\
0.006 \\
0.006 \\
0.005 \\
0.005 \\
-0.001 \\
-0.003 \\
-0.004 \\
-0.002 \\
-0.008 \\
-0.006 \\
-0.002\end{array}$ & $\begin{array}{l}0.02 \\
0.02 \\
0.01 \\
0.01 \\
0.04 \\
0.08 \\
0.03 \\
0.02 \\
0.07 \\
0.08 \\
0.14 \\
0.22 \\
0.35 \\
0.30 \\
0.33 \\
0.15 \\
0.21 \\
0.05 \\
0.09 \\
0.04 \\
0.02 \\
0.08 \\
0.05 \\
0.02\end{array}$ & $\begin{array}{l}0.689 \\
0.608 \\
0.795 \\
0.874 \\
0.521 \\
0.253 \\
0.593 \\
0.601 \\
0.159 \\
0.120 \\
0.016 \\
0.001 \\
0.000 \\
0.000 \\
0.000 \\
0.014 \\
0.002 \\
0.250 \\
0.075 \\
0.378 \\
0.558 \\
0.130 \\
0.247 \\
0.688\end{array}$ & $\begin{array}{l}47 \\
44 \\
41 \\
39 \\
36 \\
37 \\
40 \\
47 \\
55 \\
55 \\
55 \\
55 \\
55 \\
55 \\
55 \\
55 \\
55 \\
55 \\
55 \\
55 \\
53 \\
52 \\
52 \\
50\end{array}$ \\
\hline
\end{tabular}

LRD Regression Coefficients for Coastal College - Non-Standard Day - Winter

\begin{tabular}{|c|c|c|c|c|c|c|}
\hline Hour & $\begin{array}{l}\text { Reg } \\
\text { Base Load } \\
\text { Intercept } \\
\left(W / t^{2}\right)\end{array}$ & $\begin{array}{c}\text { ssion Parar } \\
\text { Dew Point } \\
\text { Coefficeint } \\
\left(W / \mathrm{ft}^{2} /{ }^{\circ} \mathrm{F}\right)\end{array}$ & 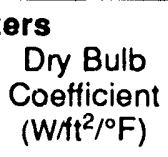 & $R^{2}$ & $\begin{array}{c}\text { Statistics } \\
\text { Significance } \\
\text { of } \\
\text { F-Statistic }\end{array}$ & $N$ \\
\hline 1 & 0.718 & -0.001 & -0.033 & 0.11 & 0.842 & 5 \\
\hline 2 & 83.998 & 0.907 & 0.644 & 1.00 & 2.000 & 0 \\
\hline 3 & 0.000 & 0.000 & 0.000 & 0.00 & 1.000 & 0 \\
\hline 4 & 0.000 & 0.000 & 0.000 & 0.00 & 1.000 & 0 \\
\hline 5 & 0.000 & 0.000 & 0.000 & 0.00 & 1.000 & 0 \\
\hline 6 & 0.000 & 0.000 & 0.000 & 0.00 & 1.000 & 0 \\
\hline 7 & 0.000 & 0.000 & 0.000 & 0.00 & 1.000 & 0 \\
\hline 8 & 0.780 & -0.002 & -0.050 & 0.94 & 0.057 & 4 \\
\hline 9 & 1.026 & -0.019 & -0.039 & 0.49 & 0.066 & 10 \\
\hline 10 & 0.846 & -0.002 & 0.011 & 0.08 & 0.272 & 32 \\
\hline 11 & 0.863 & -0.003 & 0.007 & 0.08 & 0.181 & 42 \\
\hline 12 & 0.837 & -0.001 & 0.009 & 0.12 & 0.041 & 51 \\
\hline 13 & 0.862 & -0.001 & 0.006 & 0.09 & 0.083 & 52 \\
\hline 14 & 0.862 & 0.001 & 0.007 & 0.08 & 0.111 & 52 \\
\hline 15 & 0.876 & 0.001 & 0.006 & 0.07 & 0.168 & 54 \\
\hline 16 & 0.845 & 0.001 & 0.007 & 0.10 & 0.084 & 51 \\
\hline 17 & 0.869 & -0.004 & 0.003 & 0.09 & 0.108 & 49 \\
\hline 18 & 0.899 & -0.007 & -0.002 & 0.12 & 0.053 & 47 \\
\hline 19 & 0.902 & -0.003 & 0.002 & 0.04 & 0.455 & 39 \\
\hline 20 & 0.883 & -0.000 & 0.009 & 0.05 & 0.473 & 31 \\
\hline 21 & 0.875 & -0.000 & 0.010 & 0.04 & 0.602 & 25 \\
\hline 22 & 0.890 & -0.001 & -0.002 & 0.01 & 0.929 & 23 \\
\hline 23 & 0.802 & -0.000 & 0.004 & 0.06 & 0.717 & 13 \\
\hline$? 4$ & 0.751 & -0.002 & 0.001 & 0.09 & 0.681 & 10 \\
\hline
\end{tabular}


LRD Regression Coefficlents for Non-Coastal College - Standard Day - Summer

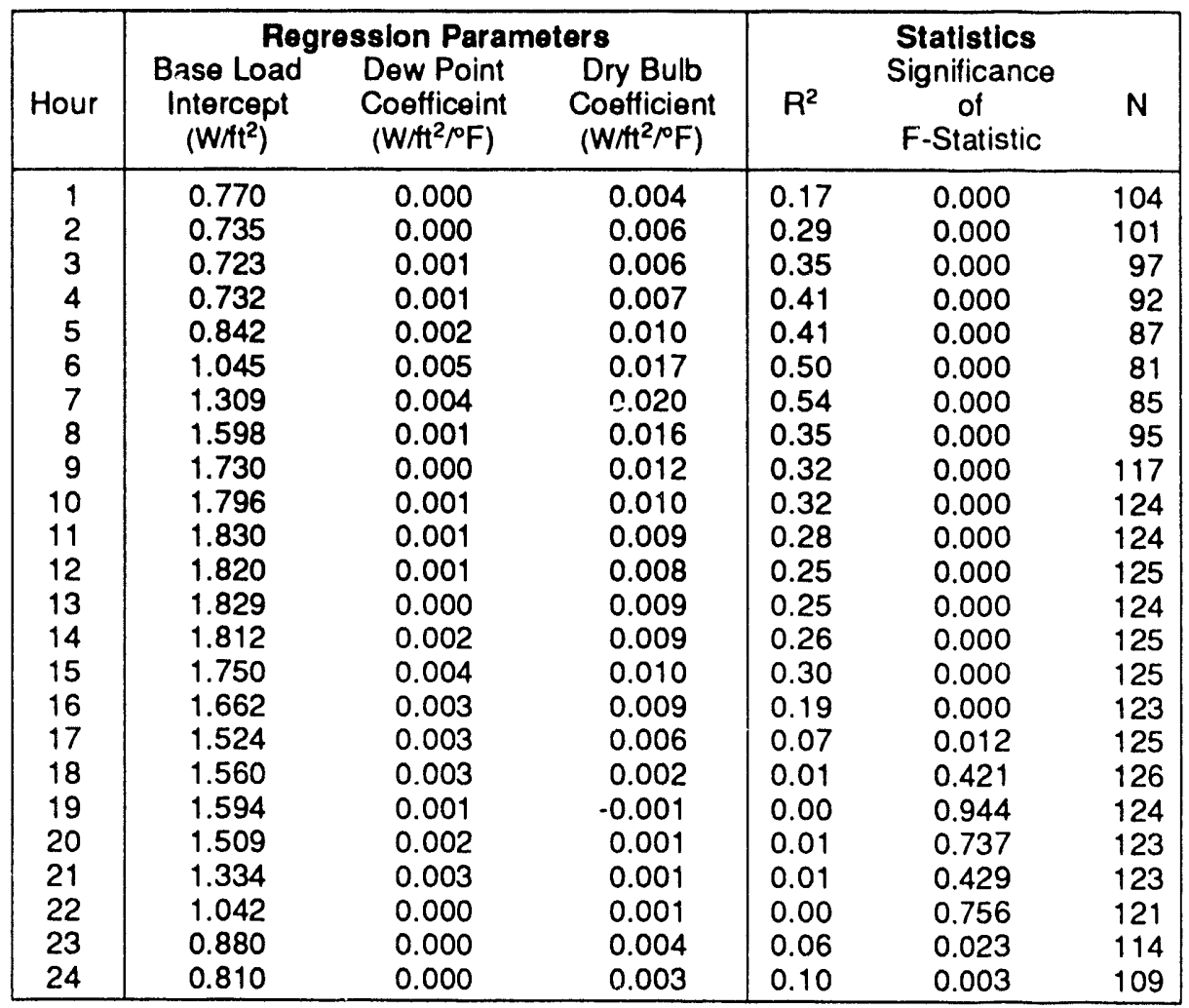

LRD Regression Coefficients for Non-Coastal College - Standard Day - Winter

\begin{tabular}{|c|c|c|c|c|c|c|}
\hline \multirow[b]{2}{*}{ Hour } & \multicolumn{3}{|c|}{ Regression Parameters } & \\
\hline & $\begin{array}{l}\text { Base Load } \\
\text { Intercept } \\
\left(W / t^{2}\right)\end{array}$ & $\begin{array}{l}\text { Dew Point } \\
\text { Coefficeint } \\
\left(\mathrm{W} / \mathrm{ft}^{2} /{ }^{\circ} \mathrm{F}\right)\end{array}$ & $\begin{array}{l}\text { Dry Bulb } \\
\text { Coefficient } \\
\left(\text { W/ft } \text { t }^{\circ} F\right)\end{array}$ & $R^{2}$ & $\begin{array}{c}\text { Significance } \\
\text { of } \\
\text { F-Statistic }\end{array}$ & $N$ \\
\hline 1 & 0.782 & 0.003 & 0.004 & 0.35 & 0.040 & 17 \\
\hline 2 & 0.750 & 0.003 & 0.004 & 0.52 & 0.006 & 16 \\
\hline 3 & 0.733 & 0.002 & 0.002 & 0.53 & 0.015 & 13 \\
\hline 4 & 0.732 & 0.002 & 0.001 & 0.30 & 0.238 & 10 \\
\hline 5 & 0.825 & -0.000 & -0.015 & 0.28 & 0.269 & 10 \\
\hline 6 & 0.923 & 0.003 & -0.002 & 0.17 & 0.383 & 12 \\
\hline 7 & 1.193 & 0.004 & -0.008 & 0.19 & 0.476 & 9 \\
\hline 8 & 1.506 & 0.008 & 0.019 & 0.31 & 0.233 & 10 \\
\hline 9 & 1.632 & 0.005 & 0.019 & 0.26 & 0.005 & 36 \\
\hline 10 & 1.764 & 0.005 & 0.006 & 0.10 & 0.045 & 62 \\
\hline 11 & 1.769 & 0.006 & 0.010 & 0.13 & 0.001 & 93 \\
\hline 12 & 1.710 & 0.010 & 0.017 & 0.27 & 0.000 & 111 \\
\hline 13 & 1.659 & 0.010 & 0.016 & 0.28 & 0.000 & 112 \\
\hline 14 & 1.667 & 0.011 & 0.016 & 0.28 & 0.000 & 116 \\
\hline 15 & 1.642 & 0.011 & 0.016 & 0.29 & 0.000 & 113 \\
\hline 16 & 1.570 & 0.011 & 0.017 & 0.29 & 0.000 & 112 \\
\hline 17 & 1.496 & 0.009 & 0.017 & 0.27 & 0.000 & 111 \\
\hline 18 & $1.43 c$ & 0.007 & 0.015 & 0.18 & 0.000 & 110 \\
\hline 19 & 1.515 & 0.008 & 0.012 & 0.13 & 0.002 & 91 \\
\hline 20 & 1.495 & 0.006 & 0.011 & 0.06 & 0.081 & 77 \\
\hline 21 & 1.526 & 0.008 & -0.007 & 0.09 & 0.067 & 57 \\
\hline 22 & 1.335 & 0.007 & -0.008 & 0.12 & 0.082 & 42 \\
\hline 23 & 1.048 & 0.004 & .0 .009 & 0.23 & 0.016 & 34 \\
\hline 24 & 0.868 & 0.004 & -0.001 & 0.32 & 0.009 & 26 \\
\hline
\end{tabular}


LRD Regression Coefficients for Non-Coastal College - Non-Standard Day - Summer

\begin{tabular}{|c|c|c|c|c|c|c|}
\hline Hour & $\begin{array}{l}\text { Reg } \\
\text { Base Load } \\
\text { Intercept } \\
\left(W / t^{2}\right)\end{array}$ & $\begin{array}{l}\text { ssion Paran } \\
\text { Dew Point } \\
\text { Coefficeint } \\
\left(\mathrm{W} / \mathrm{ft}^{2} /{ }^{\circ} \mathrm{F}\right)\end{array}$ & $\begin{array}{l}\text { ters } \\
\text { Dry Bulb } \\
\text { Coefficient } \\
\left(W / t^{2} /{ }^{\circ} F\right)\end{array}$ & $\mathrm{R}^{2}$ & $\begin{array}{c}\text { Statistics } \\
\text { Significance } \\
\text { of } \\
\text { F-Statistic }\end{array}$ & $N$ \\
\hline $\begin{array}{r}1 \\
2 \\
3 \\
4 \\
5 \\
6 \\
7 \\
8 \\
9 \\
10 \\
11 \\
12 \\
13 \\
14 \\
15 \\
16 \\
17 \\
18 \\
19 \\
20 \\
21 \\
22 \\
23 \\
24\end{array}$ & $\begin{array}{l}0.717 \\
0.695 \\
0.684 \\
0.678 \\
0.679 \\
0.681 \\
0.730 \\
0.790 \\
0.962 \\
0.989 \\
1.004 \\
0.999 \\
0.988 \\
0.996 \\
1.030 \\
0.999 \\
0.942 \\
0.975 \\
1.007 \\
0.987 \\
0.942 \\
0.857 \\
0.798 \\
0.748\end{array}$ & $\begin{array}{l}0.001 \\
0.001 \\
0.001 \\
0.001 \\
0.003 \\
0.005 \\
0.004 \\
0.005 \\
-0.005 \\
-0.005 \\
-0.007 \\
-0.008 \\
-0.009 \\
-0.009 \\
-0.007 \\
-0.007 \\
-0.006 \\
-0.010 \\
-0.010 \\
-0.005 \\
-0.004 \\
-0.001 \\
-0.001 \\
-0.002\end{array}$ & $\begin{array}{l}0.003 \\
0.003 \\
0.004 \\
0.004 \\
0.004 \\
0.008 \\
0.010 \\
0.009 \\
-0.000 \\
0.004 \\
0.005 \\
0.006 \\
0.007 \\
0.006 \\
0.004 \\
0.004 \\
0.004 \\
0.002 \\
0.000 \\
0.002 \\
0.002 \\
0.003 \\
0.003 \\
0.003\end{array}$ & $\begin{array}{l}0.09 \\
0.09 \\
0.11 \\
0.12 \\
0.20 \\
0.30 \\
0.24 \\
0.17 \\
0.04 \\
0.07 \\
0.15 \\
0.23 \\
0.24 \\
0.25 \\
0.19 \\
0.22 \\
0.22 \\
0.32 \\
0.32 \\
0.12 \\
0.13 \\
0.13 \\
0.10 \\
0.10\end{array}$ & $\begin{array}{l}0.126 \\
0.149 \\
0.094 \\
0.080 \\
0.019 \\
0.002 \\
0.006 \\
0.022 \\
0.395 \\
0.160 \\
0.012 \\
0.001 \\
0.001 \\
0.001 \\
0.004 \\
0.001 \\
0.002 \\
0.000 \\
0.000 \\
0.039 \\
0.027 \\
0.034 \\
0.092 \\
0.095\end{array}$ & $\begin{array}{l}45 \\
43 \\
41 \\
40 \\
37 \\
37 \\
40 \\
43 \\
53 \\
55 \\
55 \\
55 \\
55 \\
54 \\
55 \\
54 \\
55 \\
55 \\
55 \\
55 \\
54 \\
52 \\
48 \\
45\end{array}$ \\
\hline
\end{tabular}

LRD Regression Coefficients for Non-Coastal College - Non-Standard Day - Winter

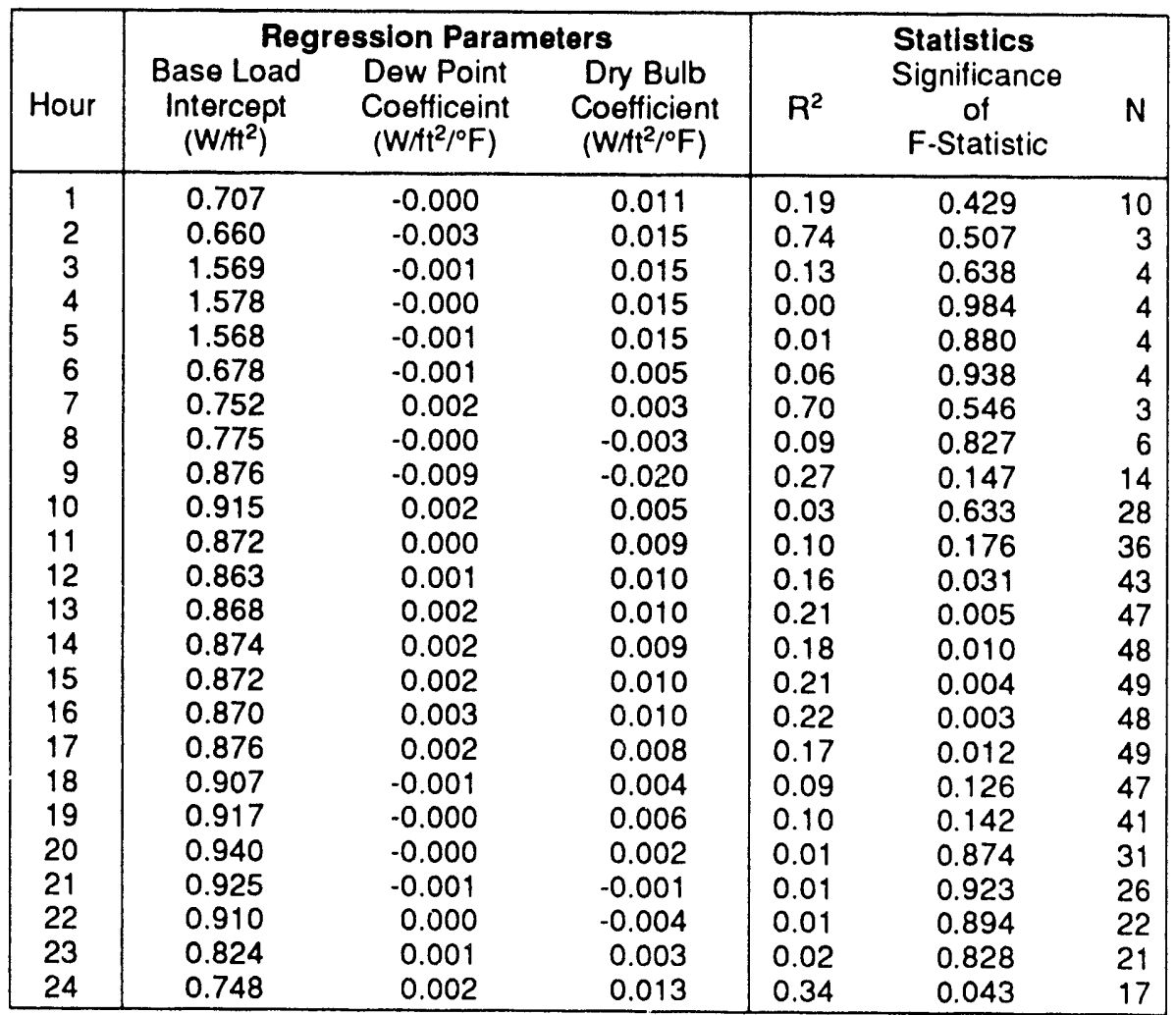


Appendix E. THI Matrices for Cooling 


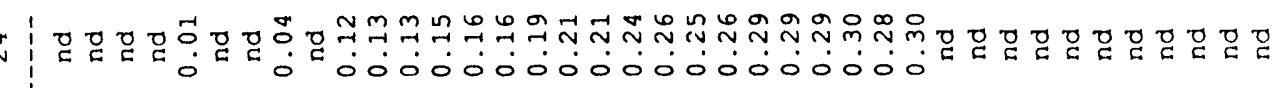

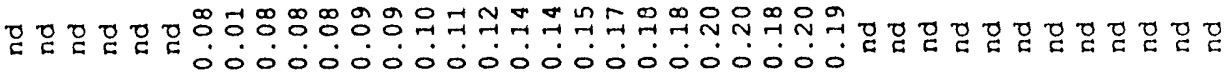

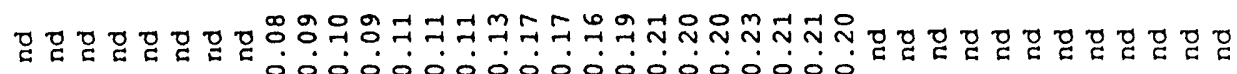

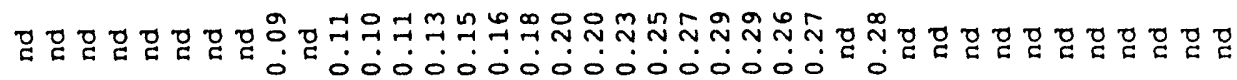

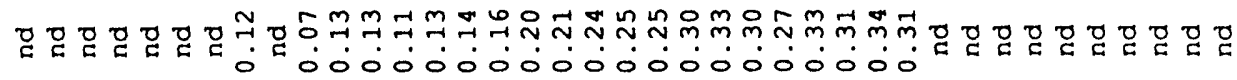

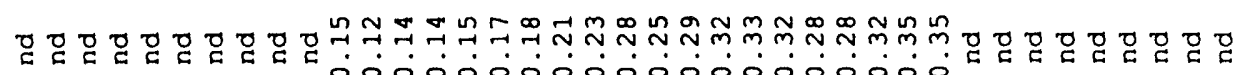

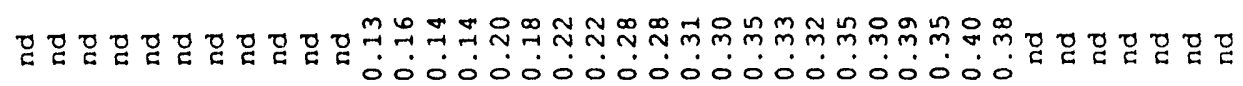

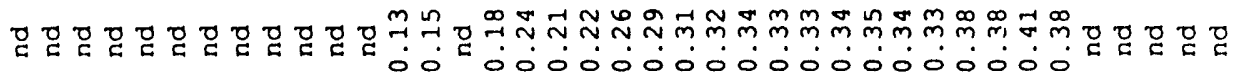

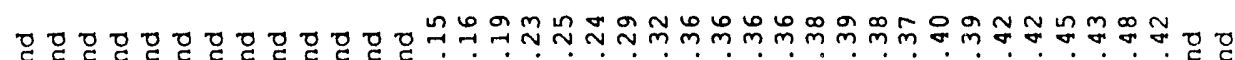

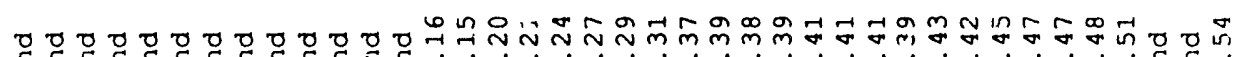
1 0000000000000000000000 o

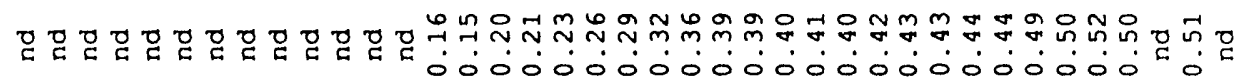

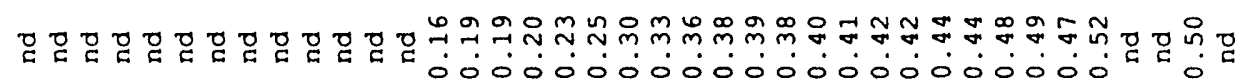

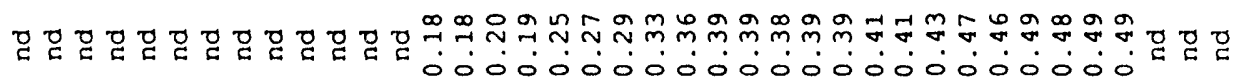

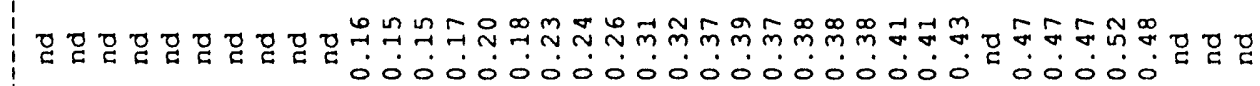

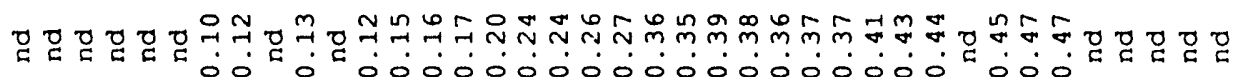

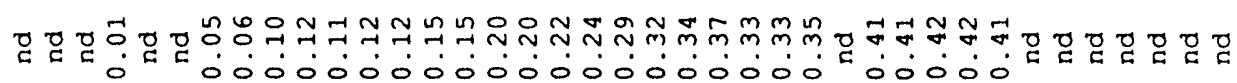

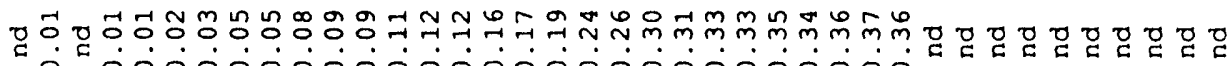

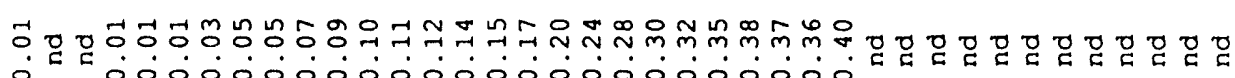

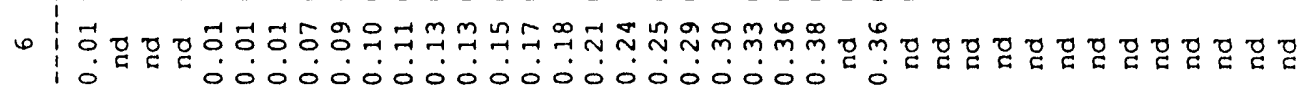

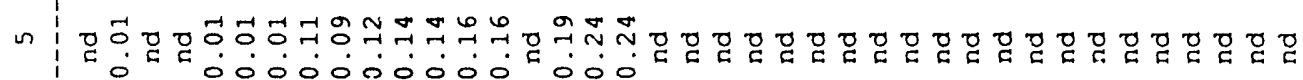

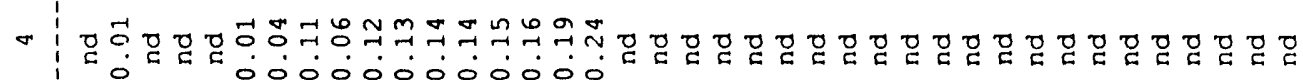

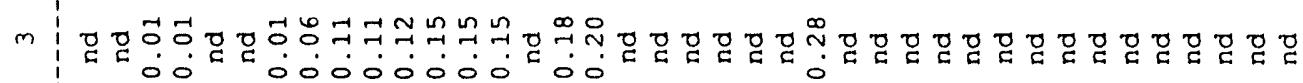

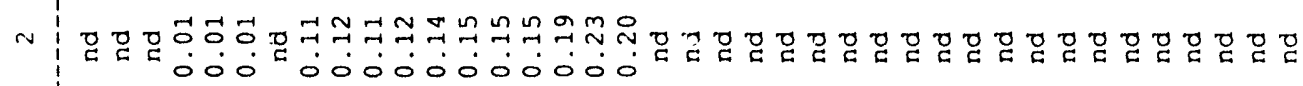

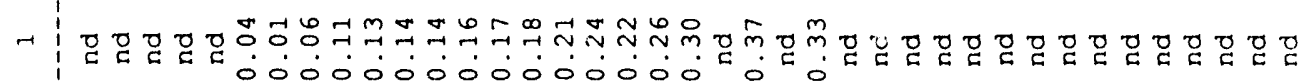
委 


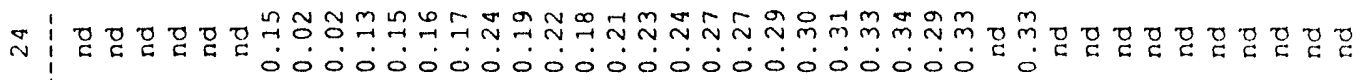

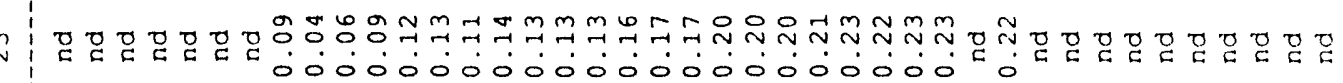

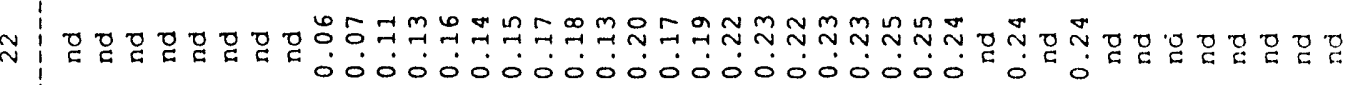
$\approx$ 듣

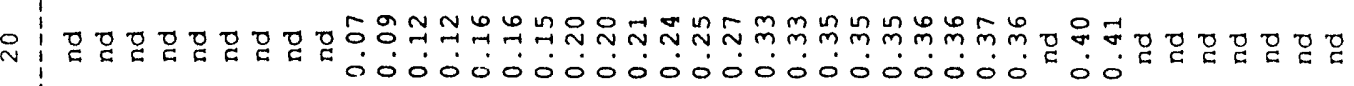

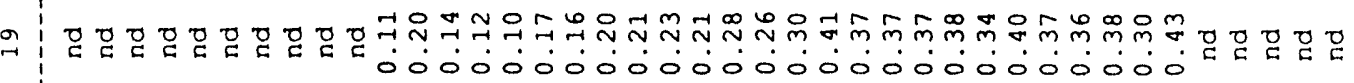

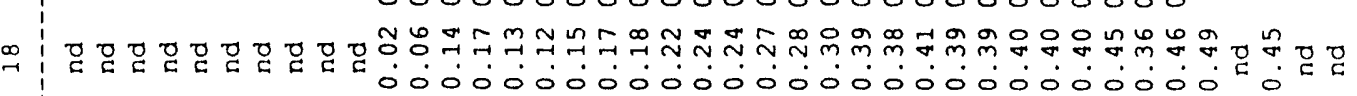

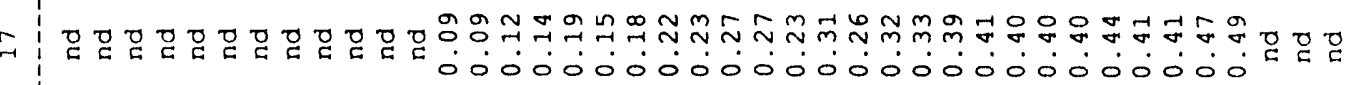

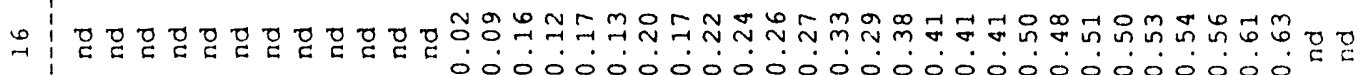

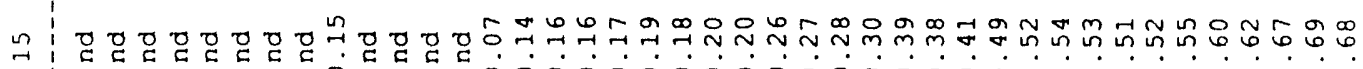

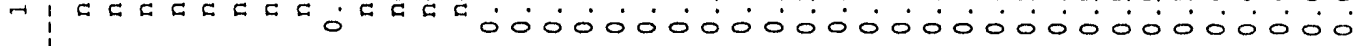

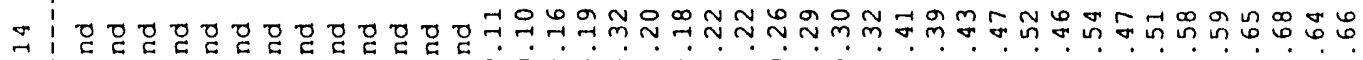

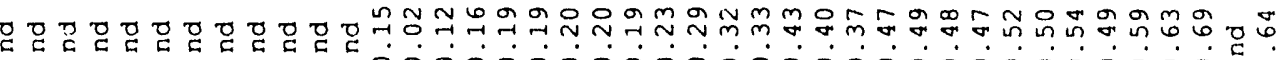

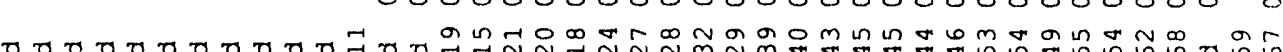

is

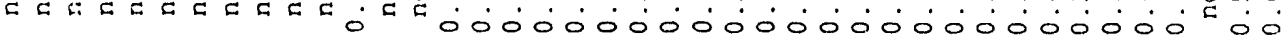

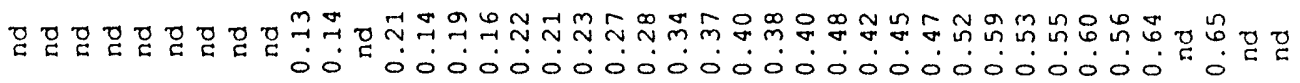

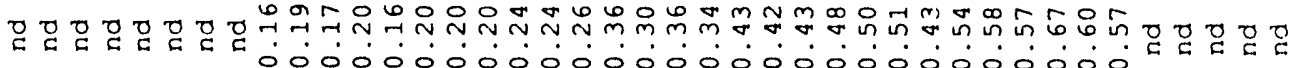
o

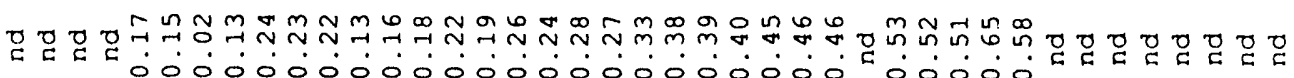

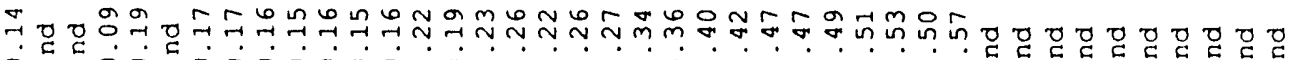
- 00 0000000000000000000000000

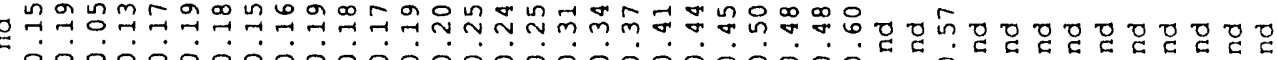

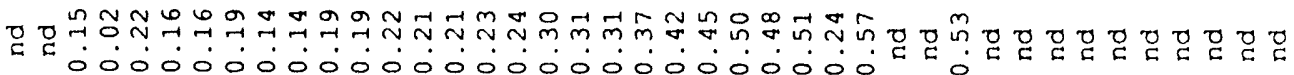

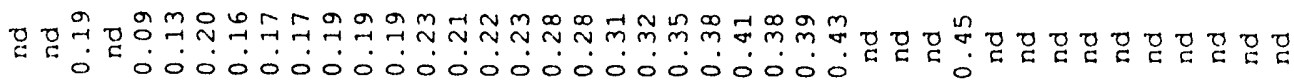

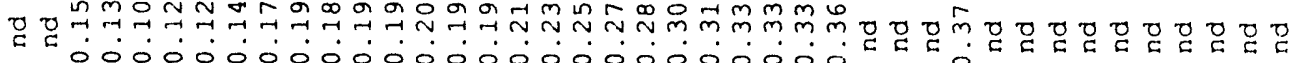

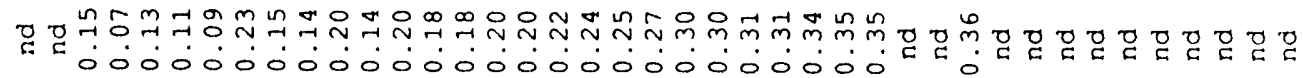

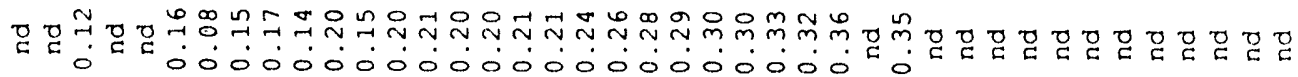
$\rightarrow$

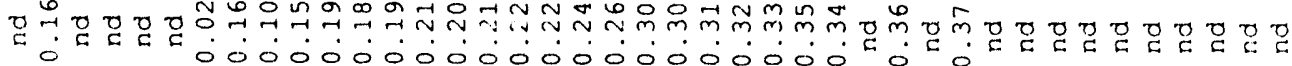

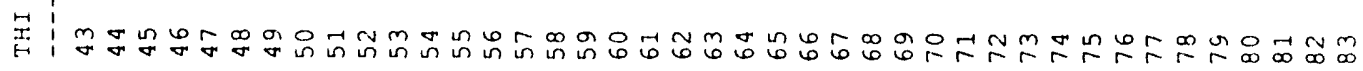


N

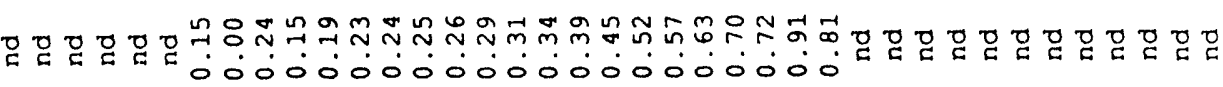

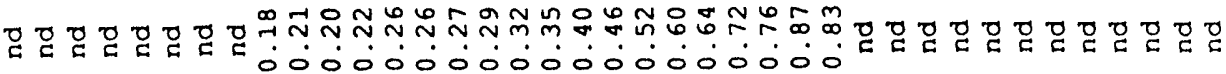

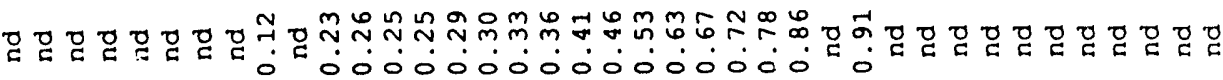

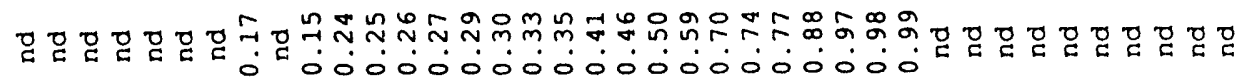

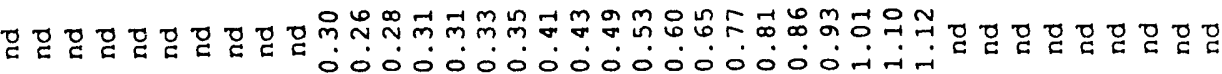

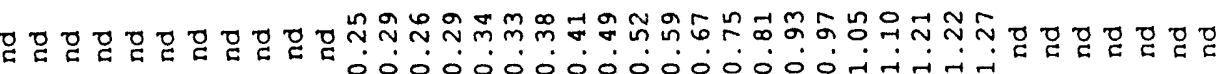

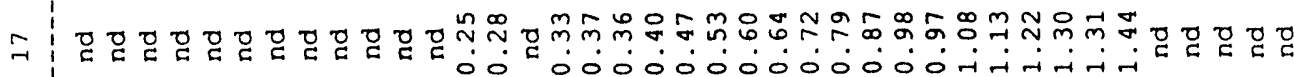

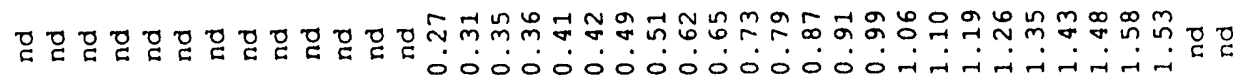

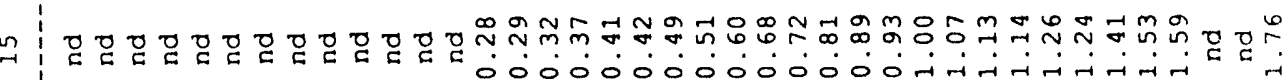

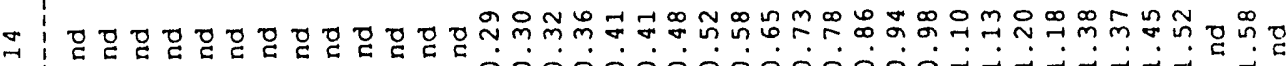

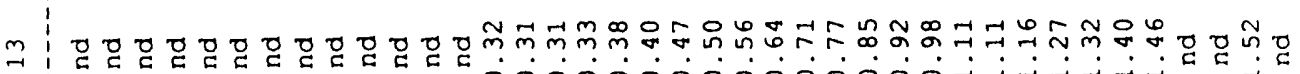

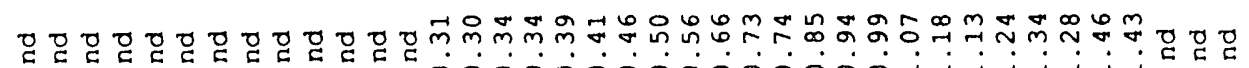
ग

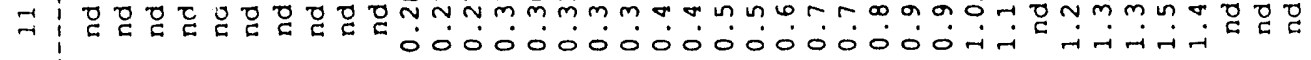

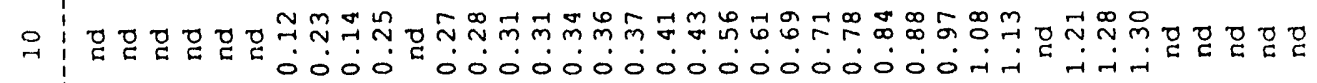

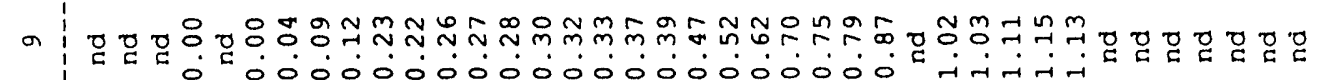

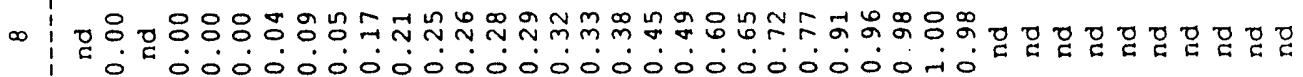

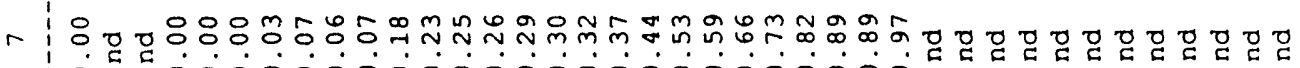
- $\begin{aligned} & 0 \\ & 0 \\ & 0\end{aligned}$

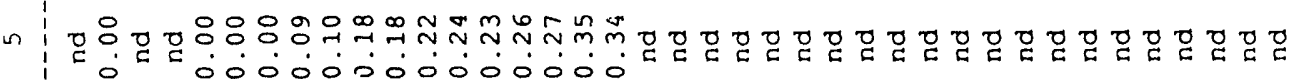
-

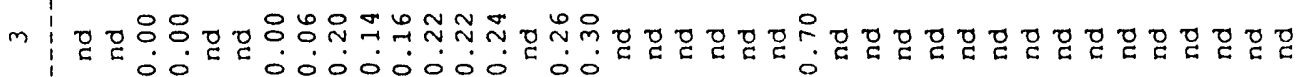
๙

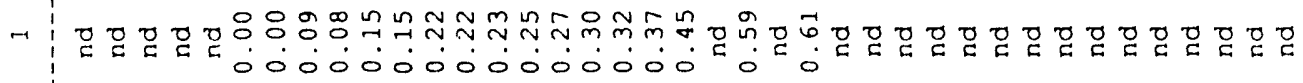

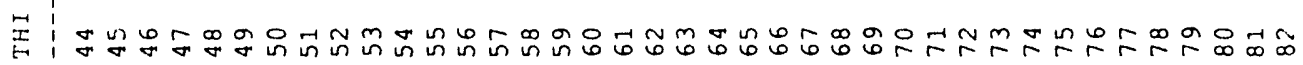




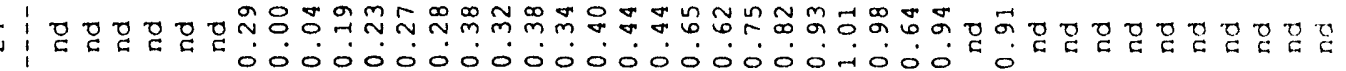

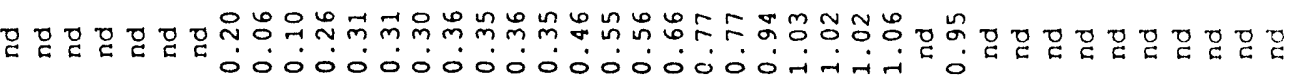

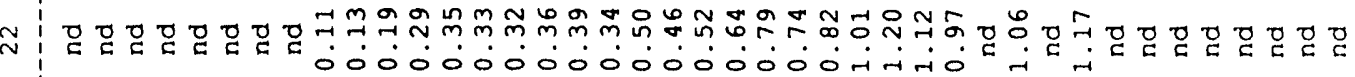

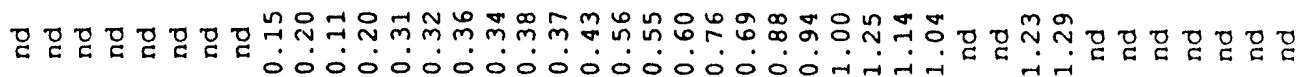

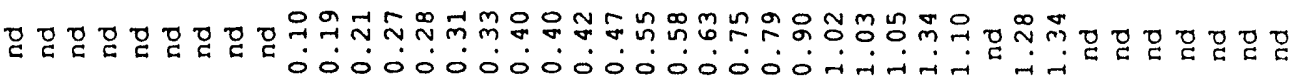

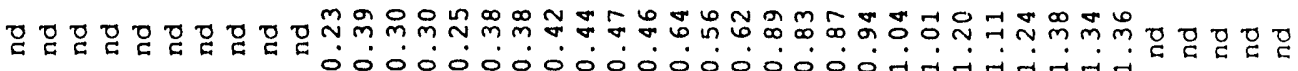

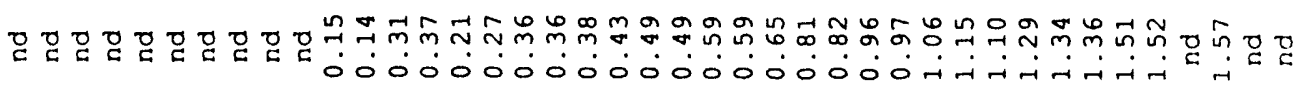

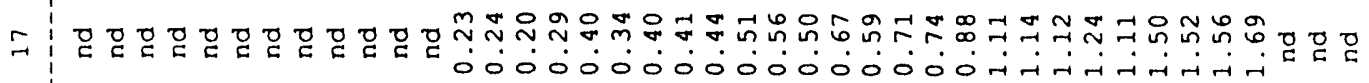

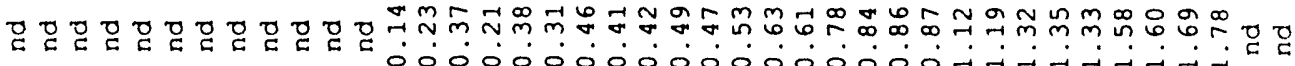

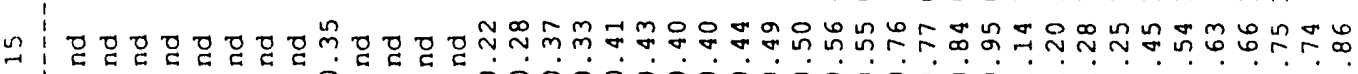

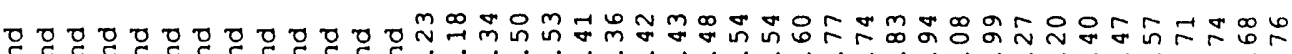

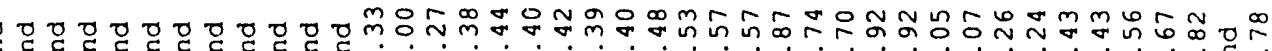

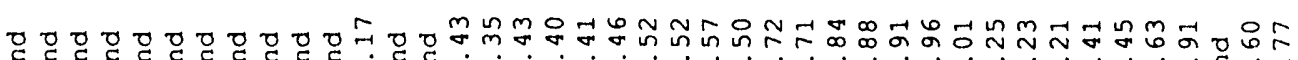

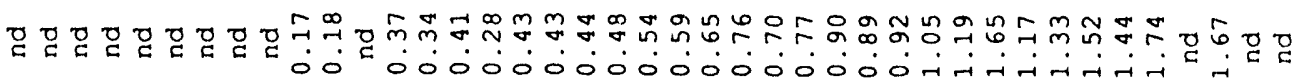

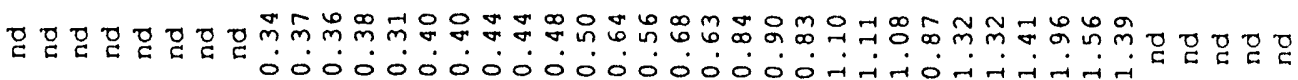
a

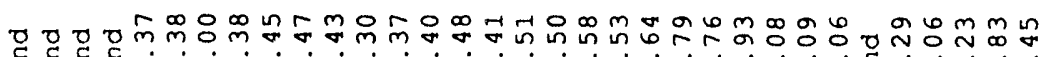

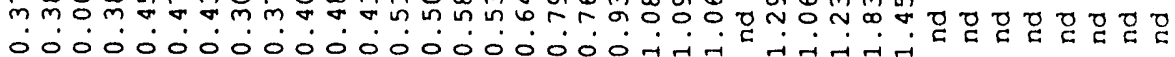

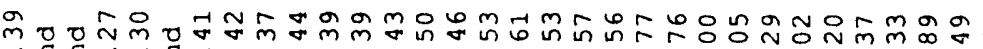

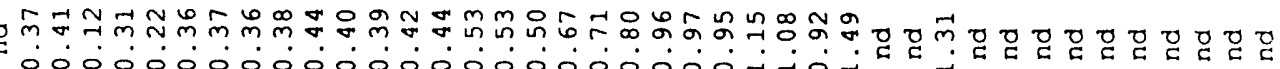

00000000000000000000000 ili

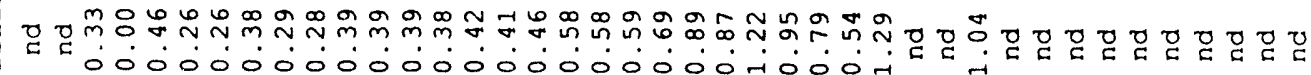

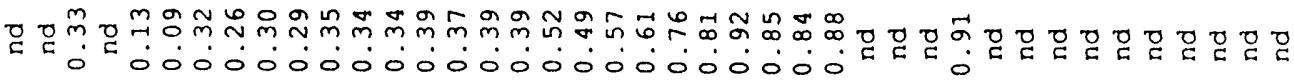

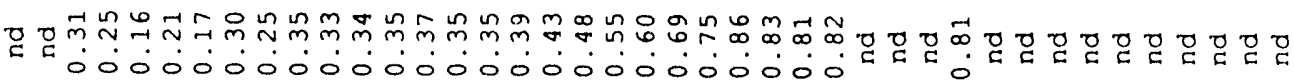
б б

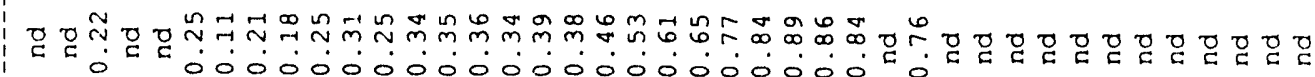
$\rightarrow$

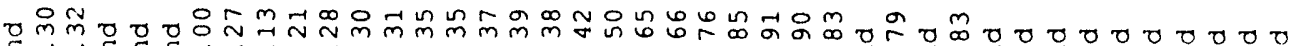

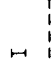

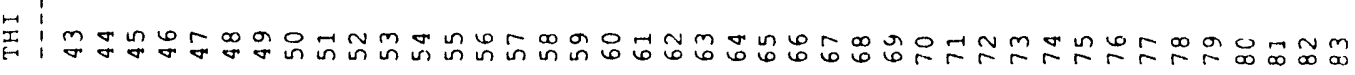




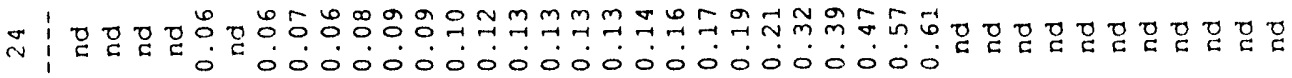
$\stackrel{m}{\sim}$\begin{tabular}{c}
0 \\
\hline
\end{tabular}

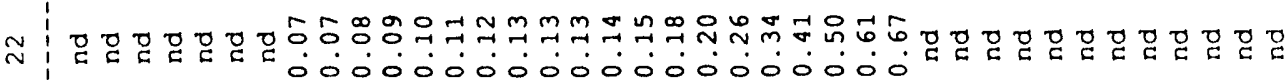

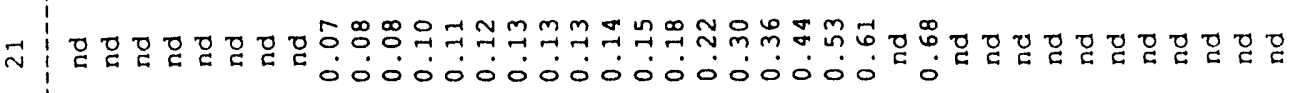

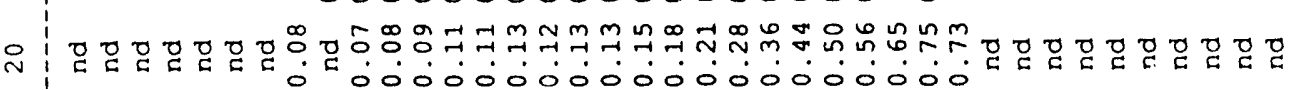

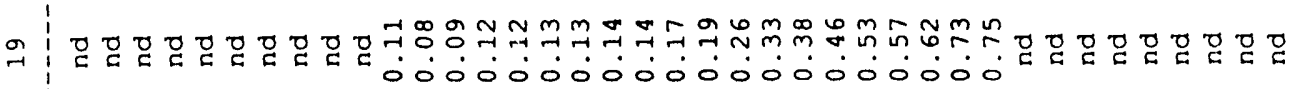

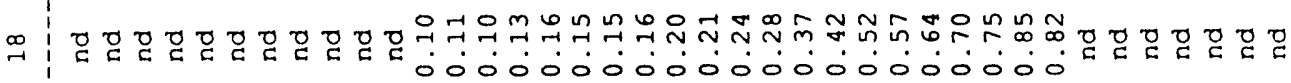

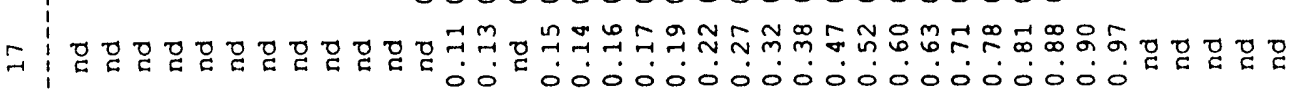

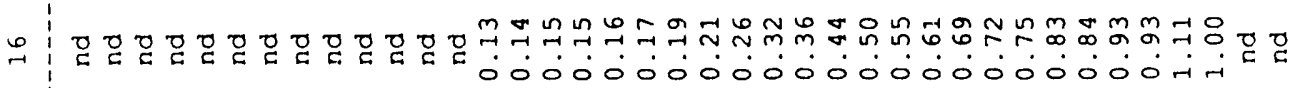

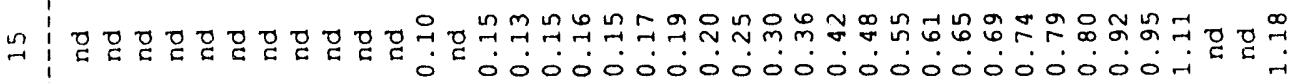

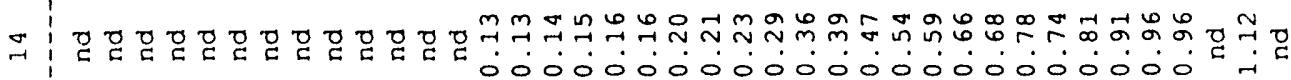

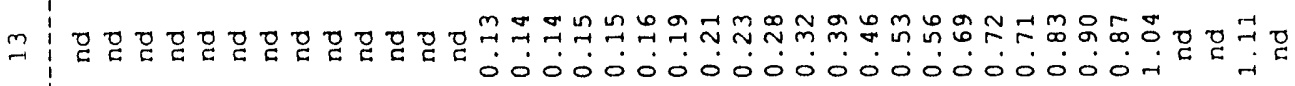

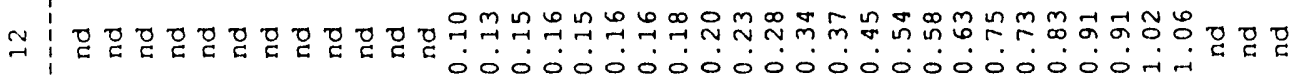

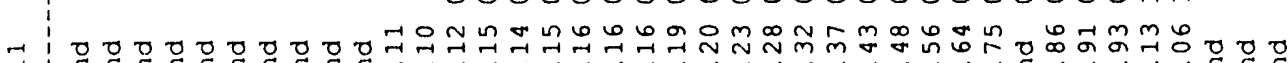

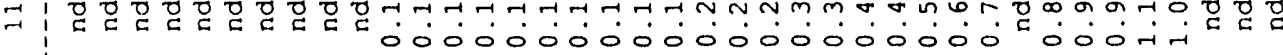

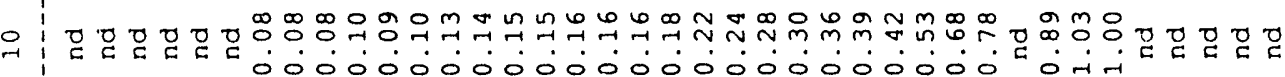

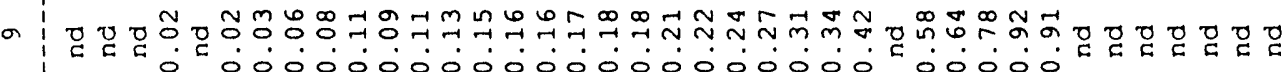

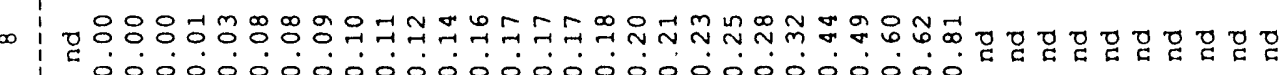
-

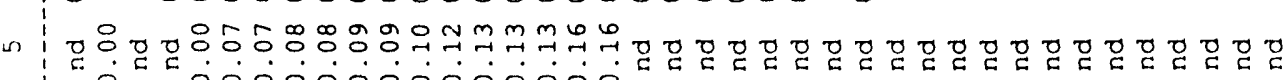
- $\begin{aligned} & 0 \\ & 0 \\ & 0\end{aligned}$

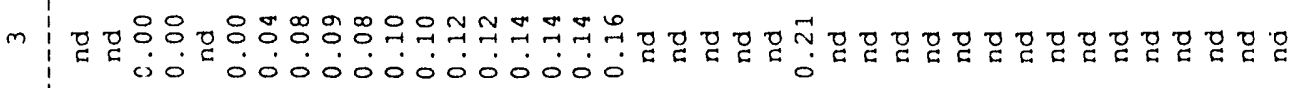

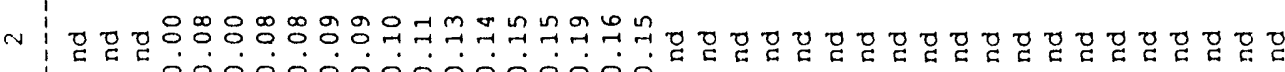

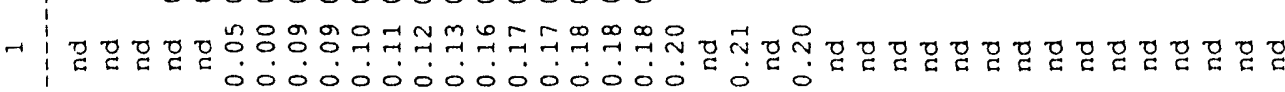

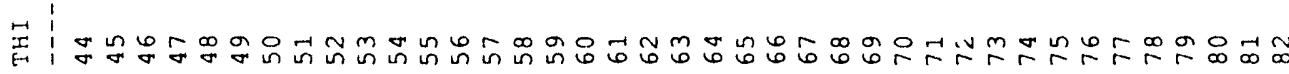




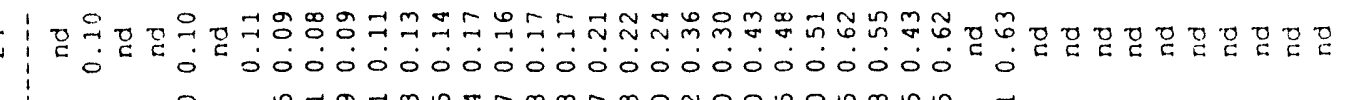

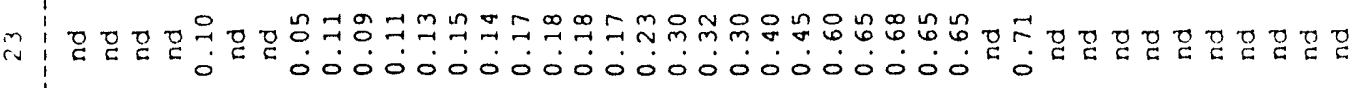

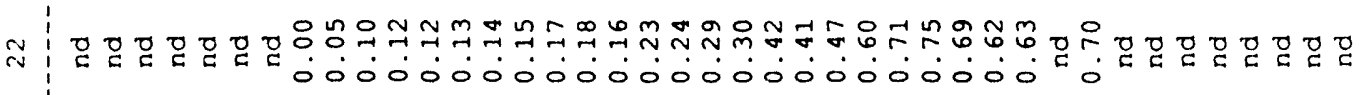

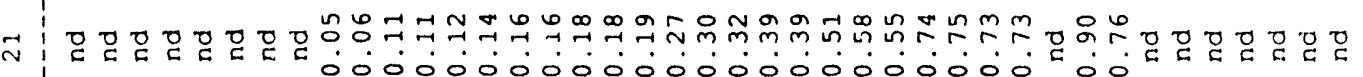

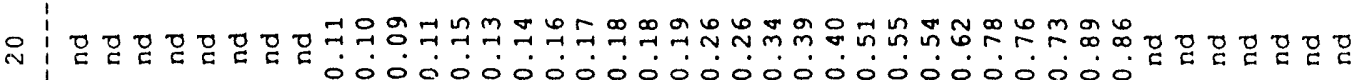

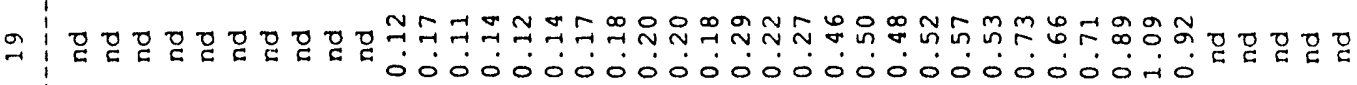

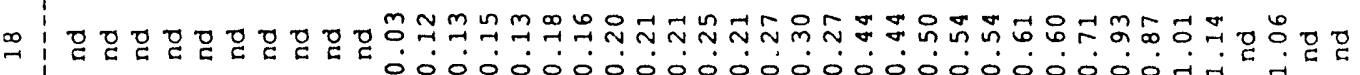

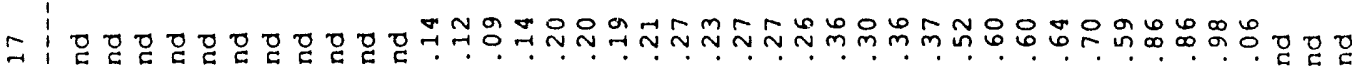

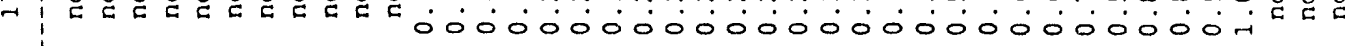

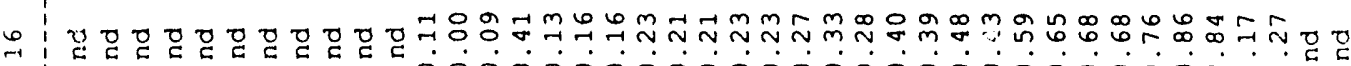
1 a00000000000000000000000007-

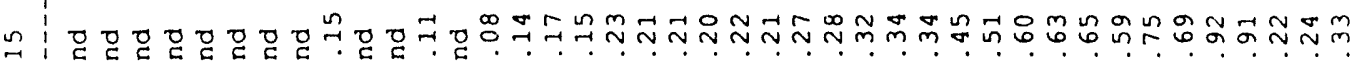

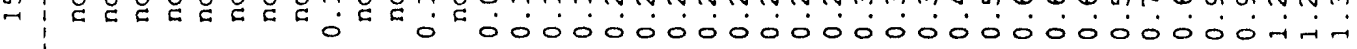

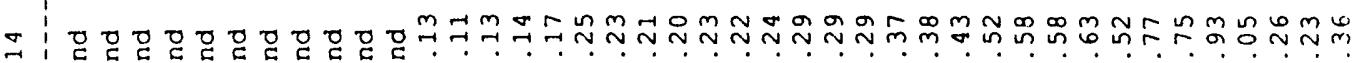

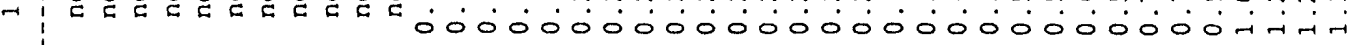

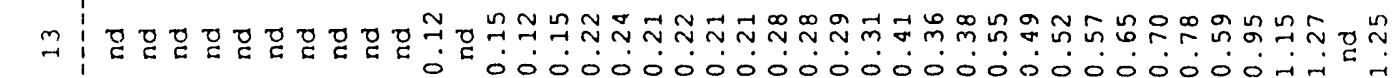

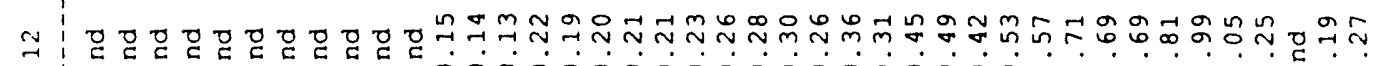

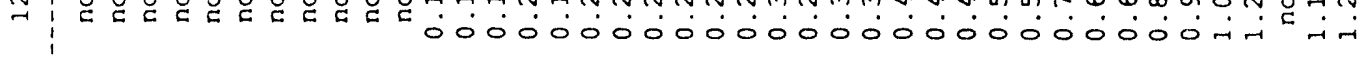

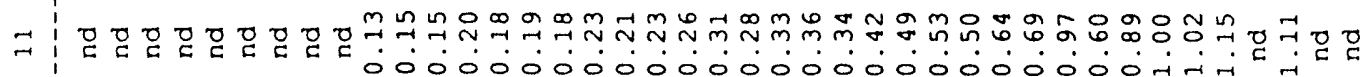

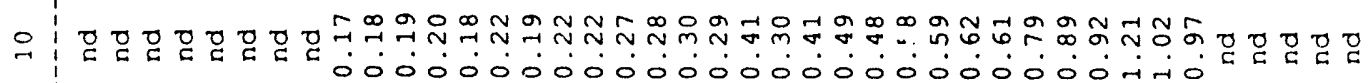

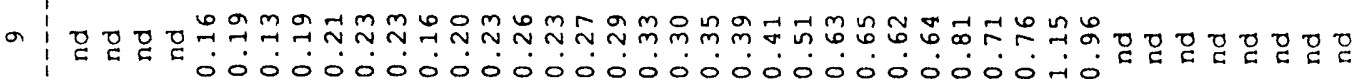

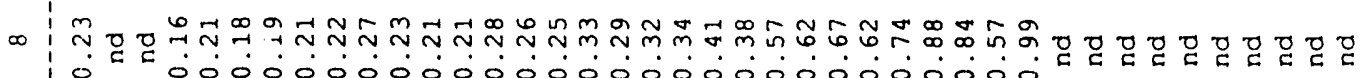
(n)

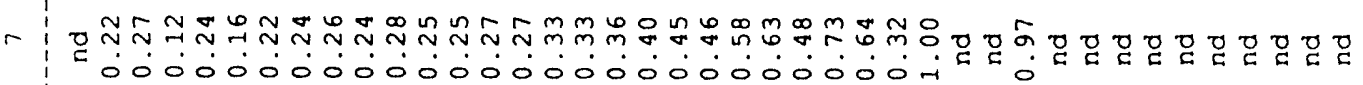

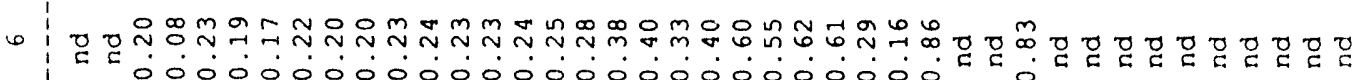

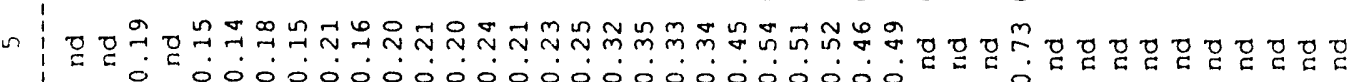

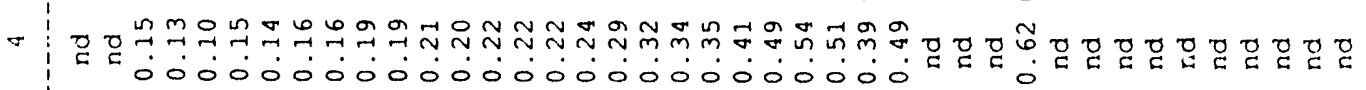

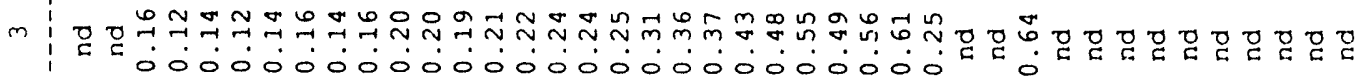
$\sim \mid \begin{aligned} & 0 \\ & 0 \\ & 0\end{aligned}$

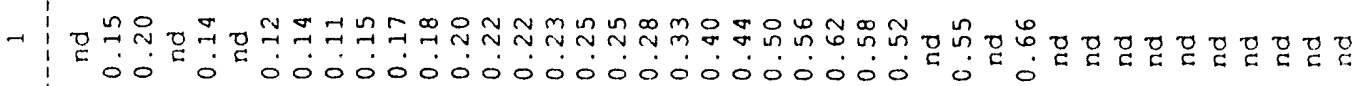

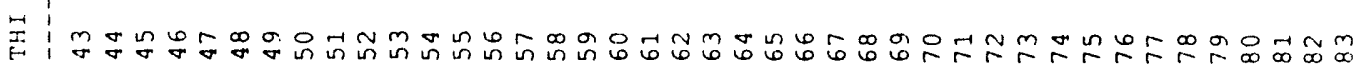




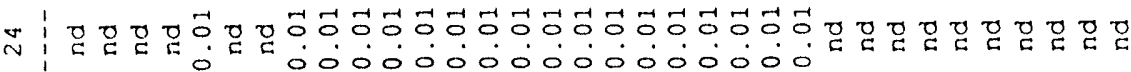

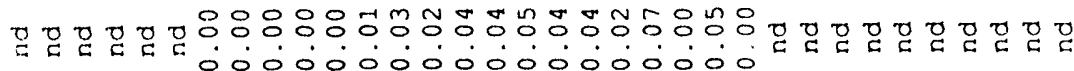

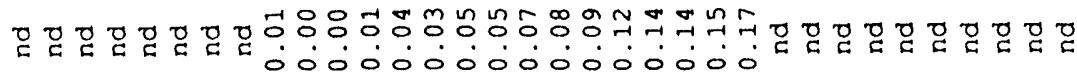

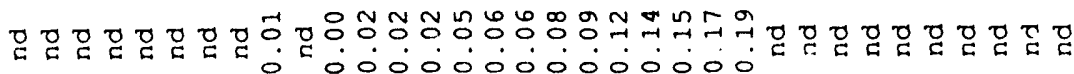

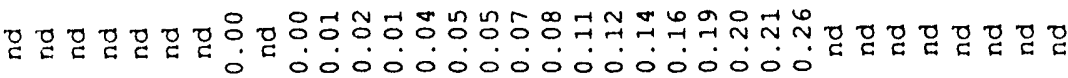

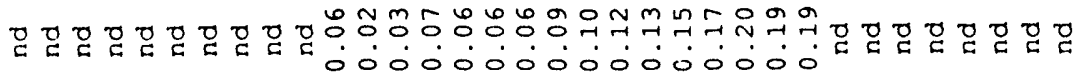

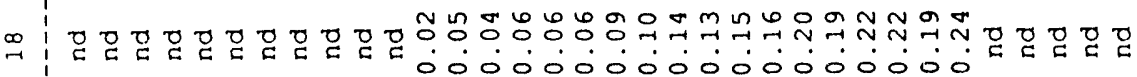

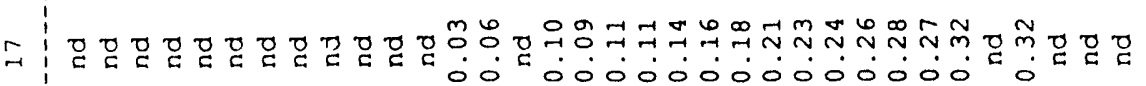

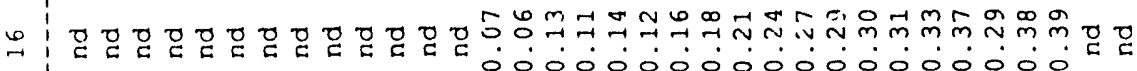
(1)

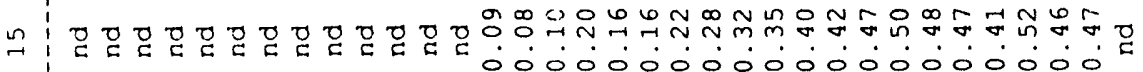
- б б

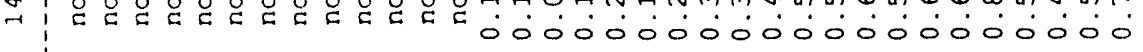

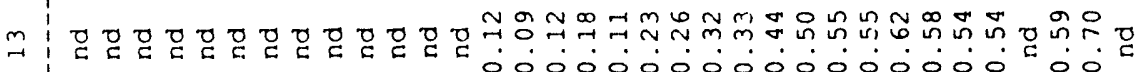

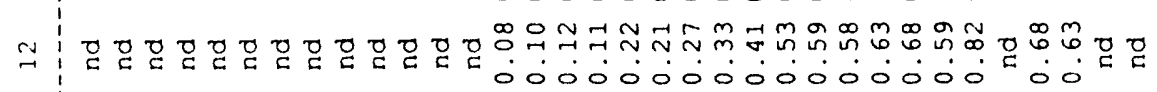

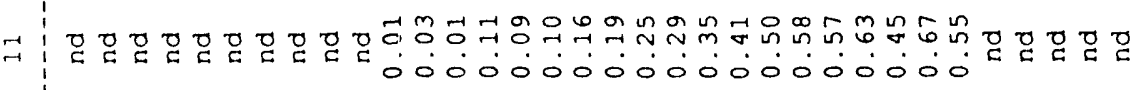

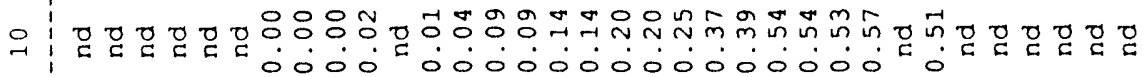

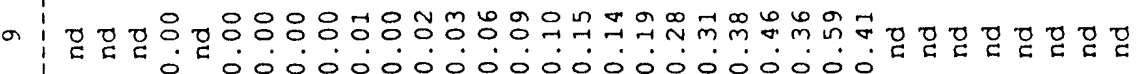

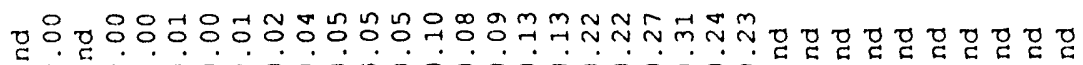

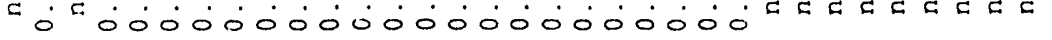

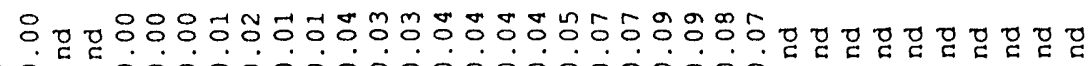
0 00000000000000000000

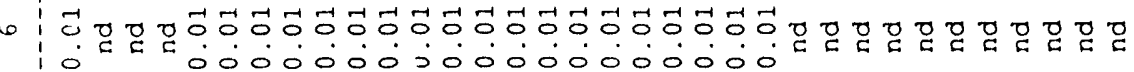

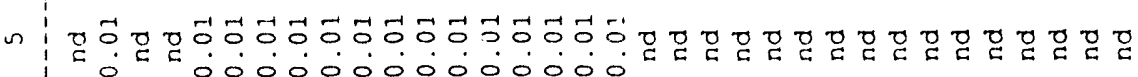

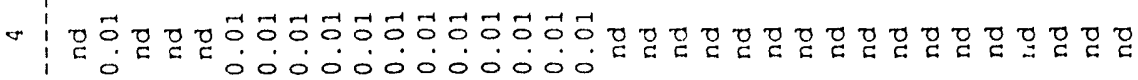
m б

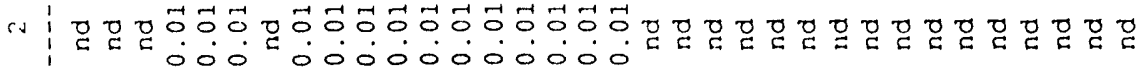

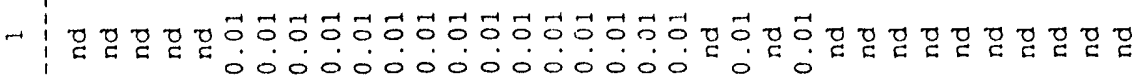

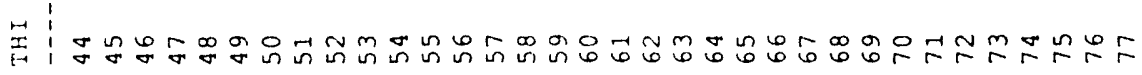




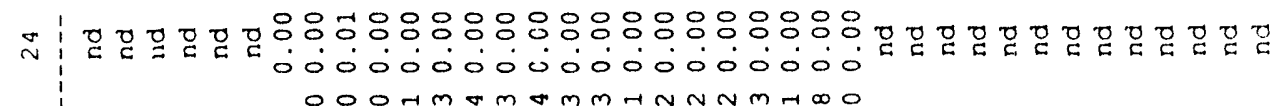

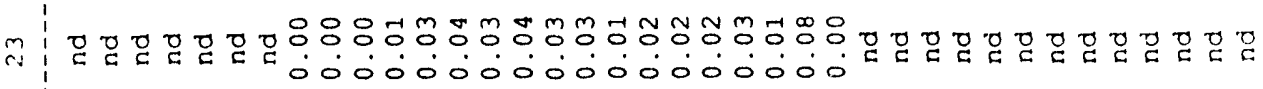

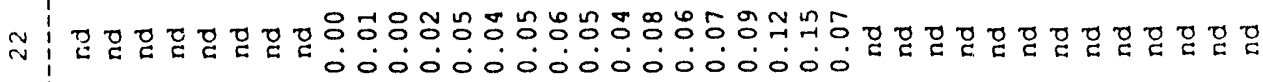

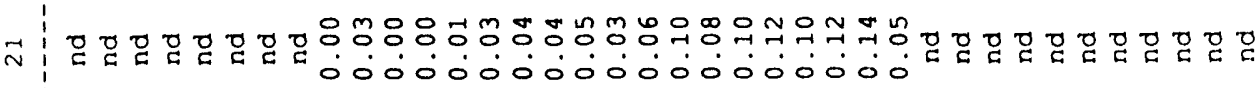

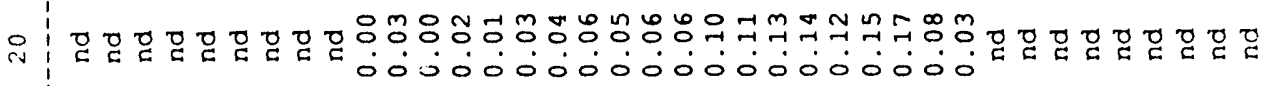

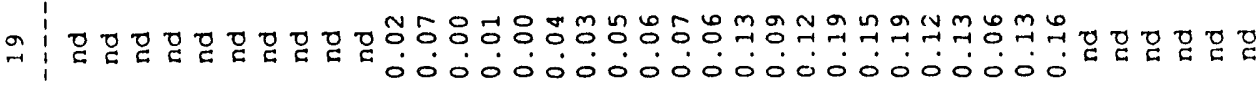

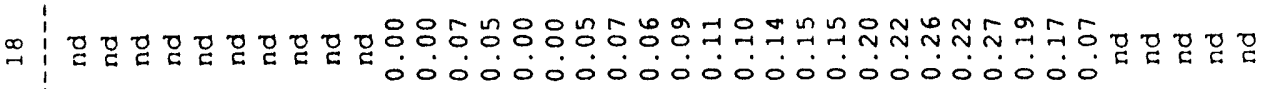

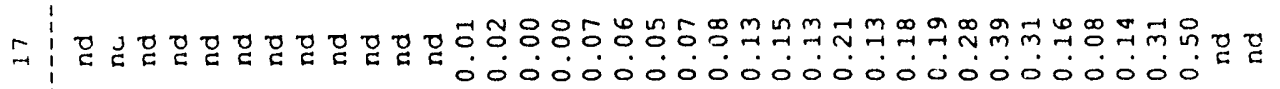

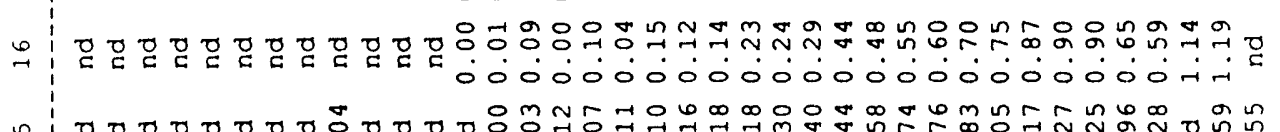

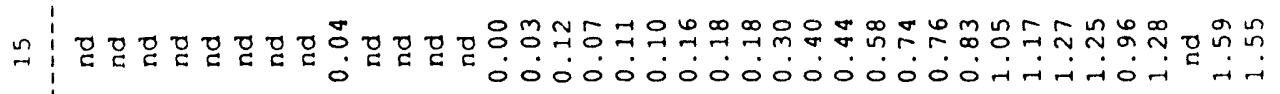

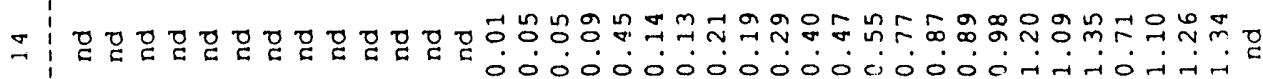$$
\Rightarrow
$$

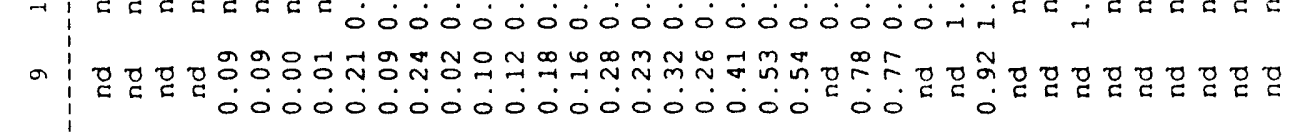

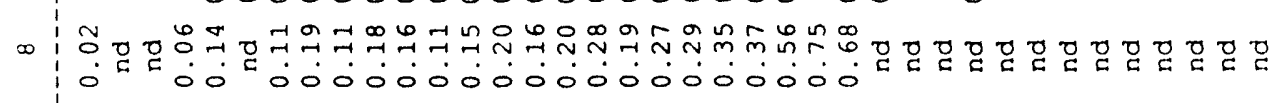

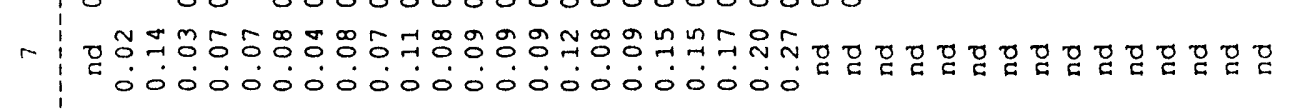

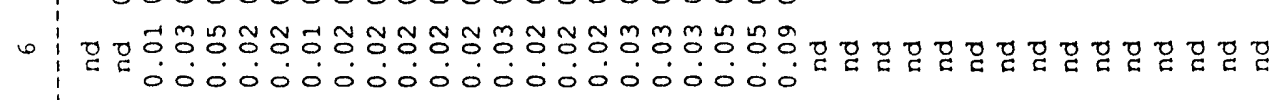

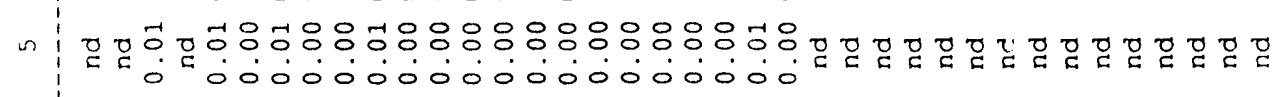

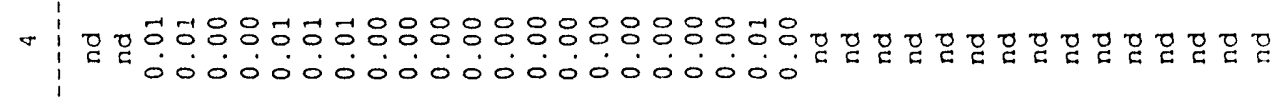

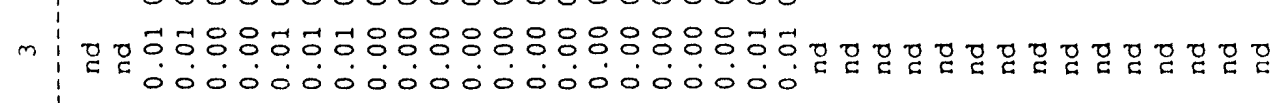

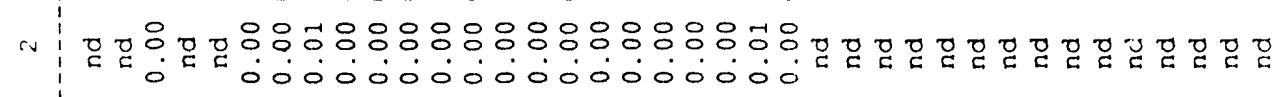

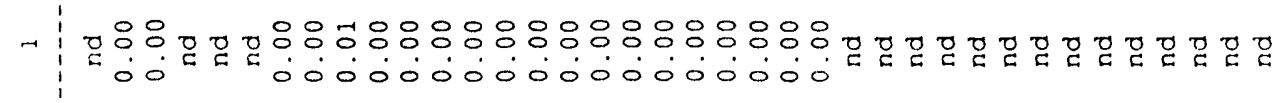

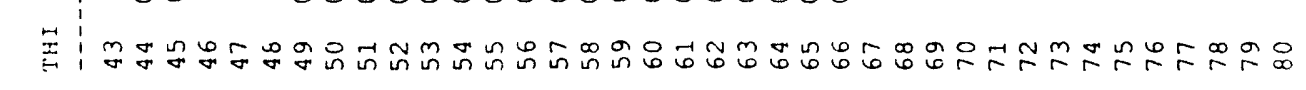

\section{7}



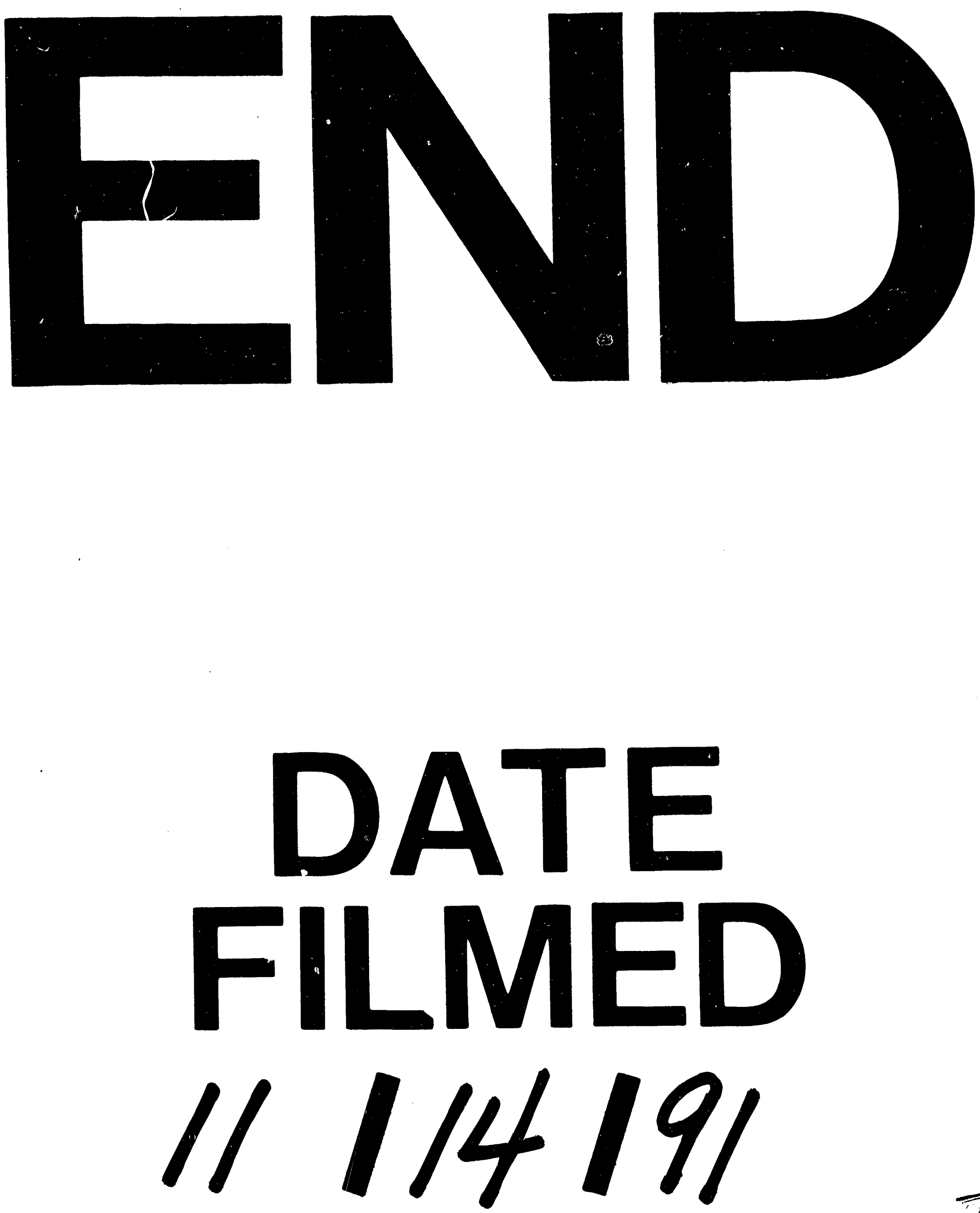
